NBSIR 84-3001

\title{
EFFECT OF CRACK-TIP REGION CONSTRAINT ON FRACTURE IN THE DUCTILE-TO-BRITTLE TRANSITION
}

National Bureau of Standards U.S. Department of Commerce Boulder, Colorado 80303

May 1984

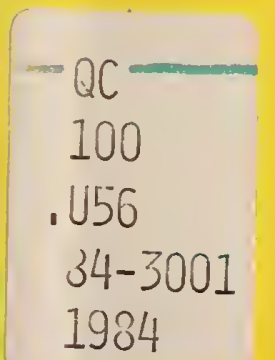





\section{EFFECT OF CRACK-TIP REGION CONSTRAINT ON FRACTURE IN THE DUCTILE-TO-BRITTLE TRANSITION}

\section{T.L. Anderson}

Fracture and Deformation Division Center for Materials Science National Bureau of Standards U.S. Department of Commerce Boulder, Colorado 80303

May 1984

Sponsored by:

U.S. Department of Interior Minerals Management Service 12203 Sunrise Valley Drive

Reston, Virginia 22091

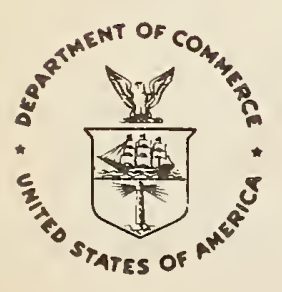





\section{FOREWORD}

The research results and analysis reported here have implications regarding the evaluation of toughness requirements of structural materials for offshore platforms.

This study shows that specimen geometry has a significant effect on the measured fracture toughness in the ductile-to-brittle transition region. The geometry effects have been attributed primarily to constraint effects. Both an increase in the specimen thickness and a decrease in the 1 igament size result in an increase in contraint at the crack-tip and therefore raise the ductile-to-brittle transition temperature.

The crack-tip region constraint (CTRC) decreases with crack blunting by a relaxation mechanism. In this study a model for the constraint relaxation has been developed, which can be used to predict the ductile-to-brittle transition curves for a low-carbon steel as a function of geometry.

The results of this study can be applied to verification of required toughness for large structural elements, such as those used in offshore platforms, through a temperature shift approach, by considering a required crack tip opening displacement (CTOD) in the structure at the service temperature versus a measured CTOD in a standard specimen at some test temperature. The required CTOD at the service temperature, $\delta_{C}$, can be calculated, for example from the CTOD Design Curve developed at the 
British Welding Institute (BWI), which provides a relationship between the allowable crack size and $\delta_{C}$ for a given design stress level, stress concentration factor and residual stress level. For example, consider a structural member of EH36 steel $150 \mathrm{~mm}$ thick at service temperature $-20^{\circ} \mathrm{C}$, subjected to a stress level of $75 \%$ of the yield strength. Also, assume that full-yield residual stresses are present. Suppose that a long surface fiaw $15 \mathrm{~mm}$ deep is present, oriented so that the stress field has a maximum effect. According to the BWI COD curve, a material toughness of $\delta_{c}=0.25 \mathrm{~mm}$ would be required in this case. The BWI procedure requires measurement of the material toughness using full-thickness fracture toughness specimens, $150 \mathrm{~mm}$ in this case. In order to be able to test standard-size specimens to verify the adequacy of the material toughness, such specimens have to be tested at a temperature of $\Delta T$ below the operating temperature considered. Based on the model presented in this study a shift in the ductile-to-brittle transition temperature due to the difference in thickness between the actual structure and the laboratory specimen can be predicted through an assumption about the global notch constraint factor, $L$, for the structure. The test temperature requirement for laboratory size specimens, under the assumptions about the service condition listed above is shown in Figure $I$ as $\Delta T$ versus specimen thickness for two $L$ values.

The theoretical model presented in this report predicts correct behavior trends for the change in transition curves with geometrical parameters. However, this model is oversimplified and excessively 
conservative. Another limitation of the analys is in the present form is that statistical effects are not taken into account. Because the scatter of toughness data in the transition region is known to be large, and because of the possibly overconservative nature of the predictions of the present model, this approach to material toughness validation should be verified further before it is considered for use in practice. Tests of specimens comparable in thickness to the upper range of actual structural thicknesses will be crucial to the verification of this temperature-shift approach.

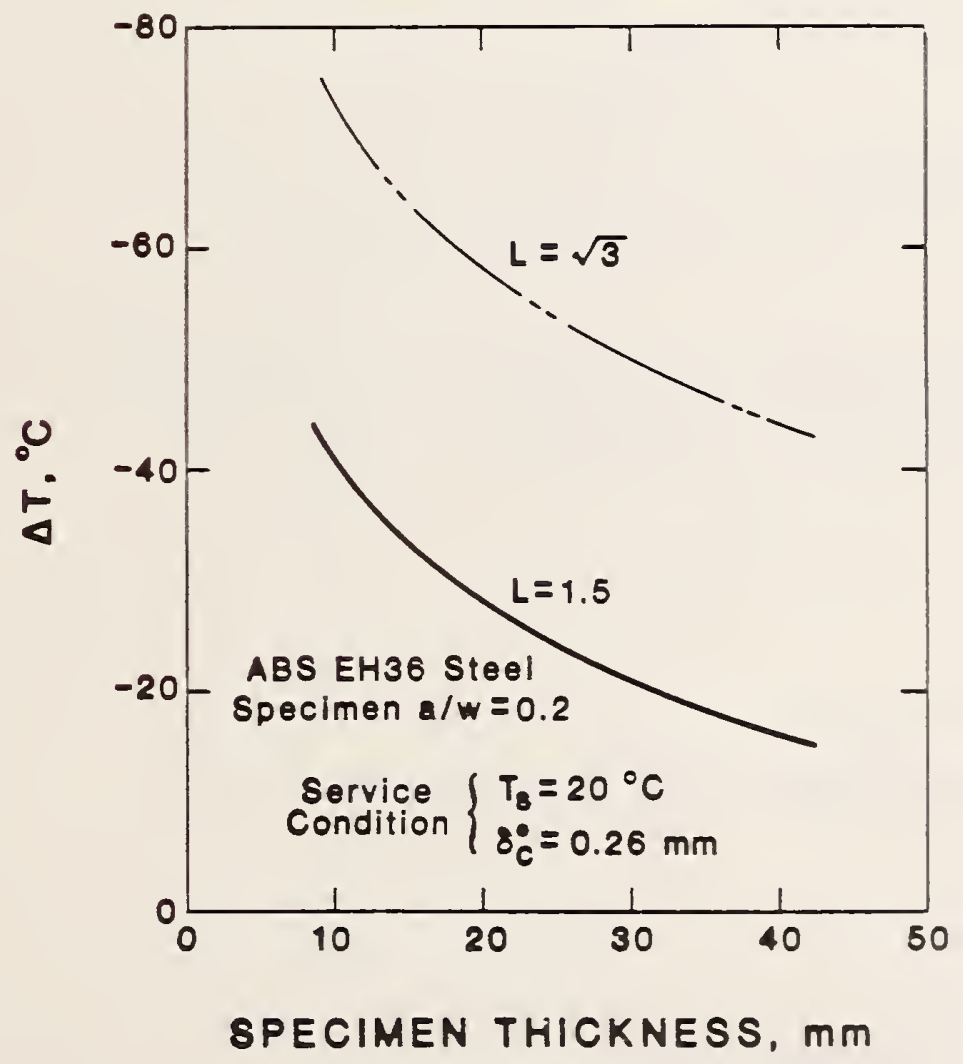

Fig. I. Temperature shift, $\Delta T$, as a function of specimen thickness, $B$, for two global notch constraint factors, L. 
FOREWORD

NOMENCLATURE

1. INTRODUCTION 1

1.1 Linear Elastic Fracture Mechanics

1.2 Elastic-Plastic Fracture Mechanics

1.2.1 The Crack-Tip Opening Displacement 8

1.2.2 J-Integral

1.2.3 Summary Comments

1.3 Micromechanisms of Crack Extension 17

1.3.1 Cleavage 19

1.3.2 Ductile Tearing (Microvoid Coalescence) 30

1.3.3 The Ductile-to-Brittle Transition 31

1.4 Effect of Specimen Size and Geometry on Fracture Behavior 35

1.4.1 Experimental Observations of the Size Effect 38

1.4.2 The Causes of Size Effects 40

1.4.3 Scaling Fracture Toughness 41

1.5 Statement of the Problem 42

1.6 Objectives 43

1.7 Technical Approach 43

2. EXPERIMENTAL PROCEDURE 46

2.1 Test Materials 46

2.2 Tensile Tests 46

2.3 Charpy V-Notch Impact Tests 48

2.4 Fracture Toughness Tests $\quad 50$

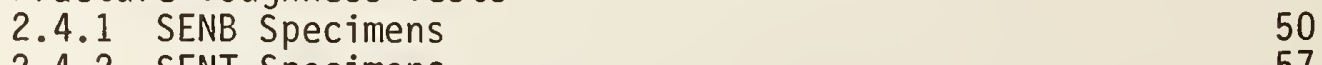

2.4.2 SENT Specimens 57

3. RESULTS AND DISCUSSION 61

3.1 Tensile Data $\quad 61$ 
3.2 Charpy-Impact Data

$\frac{\text { Page }}{64}$

3.3 Fracture Toughness 66

3.3.1 ABS EH36 Steel 66

3.3.2 Pure Iron $\quad 87$

3.3.3 Fractography and Metallography 89

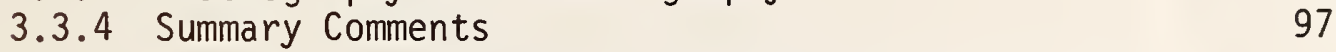

3.4 Characterization of Fracture in the Ductile-to-Brittle
Transition Region

3.4.1 The Crack-Tip Strain Function 102

3.4.2 Measurement of CTRC 110

3.4.3 A Spring Model for CTRC 114

3.4.4 A Model to Estimate $\sigma_{\mathrm{x}}^{\circ} \quad 120$

3.4.5 Predicting the Relaxation Slope 123

3.4.6 Predicting Transition Curves 129

3.4.7 Application to Structures 136

3.4.8 Application to Other Materials 140

3.5 Constraint Versus Statistical Models 151

3.5.1 Factors that Affect Constraint 152

3.5.2 Factors that Affect Statistical Shifts in Toughness 153

3.5.3 Determining the Cause of Transition Shifts 154

3.5.4 Summary Comments 157

3.6 Discussion of Results 157

3.6.1 Advances 158

3.6.2 Applications 159

3.6.3 Limitations 161

3.6.4 Summary Comments 162

4. SUMMARY AND CONCLUSIONS 163

5. ACKNOWLEDGMENTS 164

6. REFERENCES 165

7. APPENDIX I: ADVANCES IN FRACTURE TOUGHNESS TEST METHODS 171

7.1 The Rotational Factor 171

7.2 Detecting the Onset of Tearing 173

7.3 Estimation of $\mathrm{J}$ from CMOD 175

7.4 Relationships Between J and CTOD 178

7.5 The Eta Factor 181

7.6 Crack-Tip Replication 183

8. APPENDIX II: TABULATED DATA 185 
NOMENCLATURE

A

a

$a_{c}$

B

b

C

$c_{0}$

D

$D_{0}$

d

$d_{p}$

$\mathrm{DPH}$

E

$E^{\prime}$

$F_{x}$

\section{f}

G

$G_{C}$

$\mathrm{J}$

$J_{c}, J_{i}, J_{u}, J_{m}$

$\mathrm{J}_{\text {I C }}$

K

$\mathrm{k}_{\mathrm{c}}$

K IC area

crack length

critical crack length

specimen thickness

ligament length

elastic compliance

carbide thickness or radius

diameter

initial diameter

grain diameter

martensite packet diameter

diamond pyramid hardness number

Young's modulus

$E /\left(1-v^{2}\right)$

force in the $x$ direction

volume fraction

strain energy release rate

critical G value

J-contour integral

critical $J$ values

critical $J$ which meets ASTM E 813 standards

stress intensity factor

critical $K$ value

critical K which meets ASTM E 399 standards 


\begin{tabular}{|c|c|}
\hline$K_{f}$ & $K$ value in fatigue \\
\hline k & spring stiffness \\
\hline$k_{y}$ & Hall-Petch slope \\
\hline L & notch constraint factor \\
\hline$\ell$ & characteristic length along the crack front \\
\hline m & $m$ factor to relate $J$ to $C T O D$ \\
\hline$m_{p}$ & plastic m factor \\
\hline N & hardening exponent \\
\hline$n$ & work hardening exponent $(\mathrm{I} / \mathrm{N})$ \\
\hline$P$ & load \\
\hline$P_{c}$ & critical load \\
\hline$P_{L}$ & limit load \\
\hline$P_{y}$ & load at net-section yield \\
\hline q & load point displacement \\
\hline$a_{p}$ & plastic component of $q$ \\
\hline$r$ & radius \\
\hline$r_{c}$ & carbide radius \\
\hline$r_{0}$ & overall rotational factor \\
\hline$r_{p}$ & plastic rotational factor \\
\hline s & arc length along $J$ contour \\
\hline 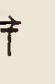 & traction vector \\
\hline U & strain energy \\
\hline$u_{p}^{v}$ & $\begin{array}{l}\text { plastic area under the load-mouth opening } \\
\text { displacement curve }\end{array}$ \\
\hline$u_{x}$ & displacement in the $x$ direction \\
\hline
\end{tabular}




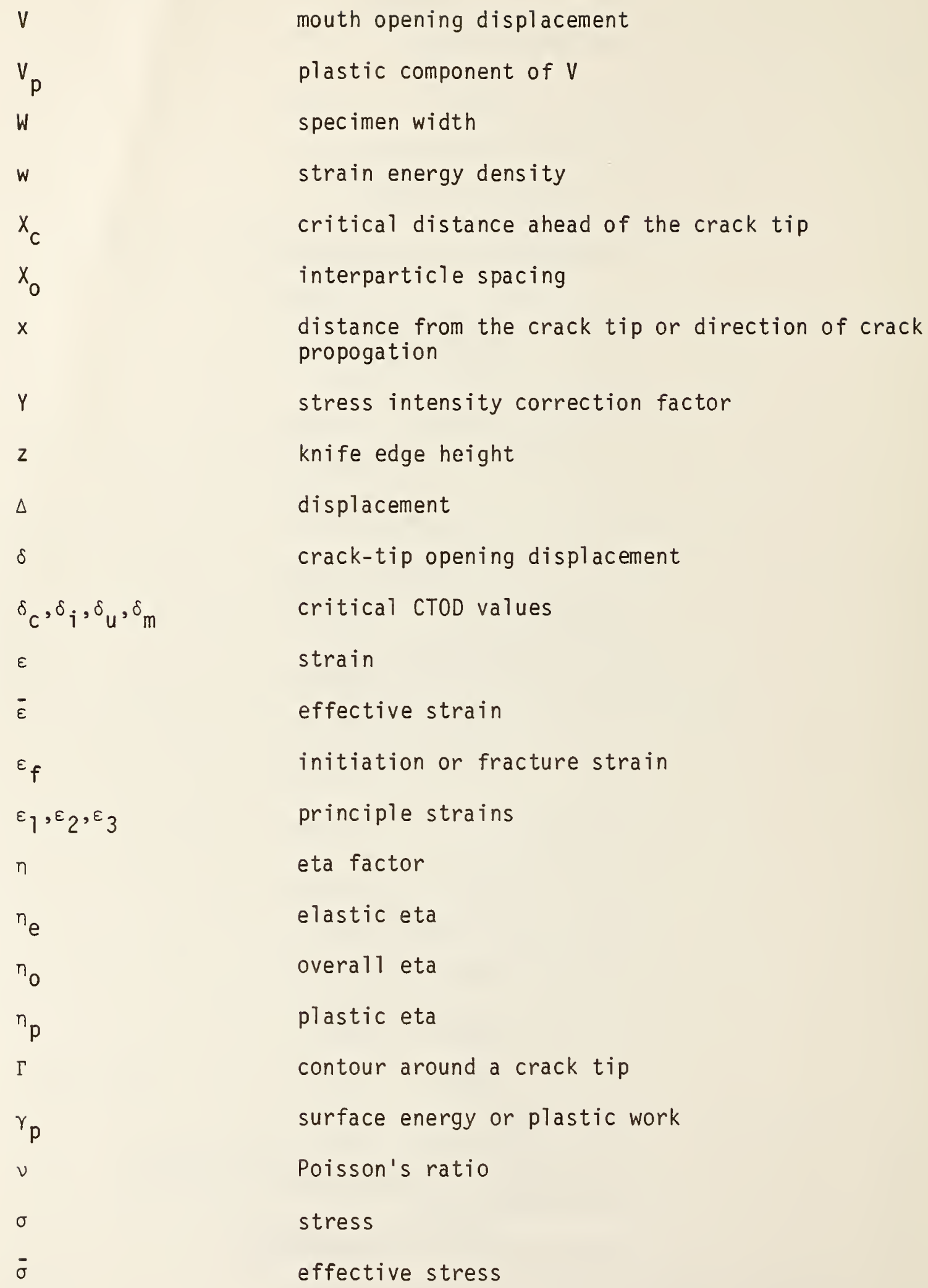

mouth opening displacement

$v_{p}$

W

w

$x_{c}$

$x_{0}$

$x$

Y

z

$\Delta$

$\delta$

$\delta_{c}, \delta_{i}, \delta_{u}, \delta_{m}$

$\varepsilon$

$\bar{\varepsilon}$

$\varepsilon_{f}$

$\varepsilon_{1}, \varepsilon_{2}, \varepsilon_{3}$

$\eta$

${ }^{n} \mathrm{e}$

$n_{0}$

$n_{p}$

$\Gamma$

$\gamma_{p}$

v

$\sigma$

$\bar{\sigma}$

plastic component of $V$

specimen width

strain energy density

critical distance ahead of the crack tip

interparticle spacing

distance from the crack tip or direction of crack propogation

stress intensity correction factor

knife edge height

displacement

crack-tip opening displacement

critical CTOD values

strain

effective strain

initiation or fracture strain

principle strains

eta factor

elastic eta

overall eta

plastic eta

contour around a crack tip

surface energy or plastic work

Poisson's ratio

stress

effective stress 


$\sigma_{f}$
$\sigma_{i j}$
$\sigma_{f l o w}$
$\sigma_{0}$
$\sigma_{x}$
$\sigma_{x}^{0}$
$\sigma_{y}$
$\sigma_{y y}$
$\sigma_{z}$
$\sigma_{1}, \sigma_{2}, \sigma_{3}$
$\tau_{e f f}$
$\tau_{i}$
$\tau_{y}$

fracture stress

stress tensor

flow stress

yield stress (lower y.s. unless stated otherwise) stress in the direction of crack propogation initial lateral stress

yield strength (upper y.s. unless stated otherwise) stress normal to the crack plane stress parallel to the crack front principle stresses effective shear stress friction stress yield shear stress 


\section{ABSTRACT}

The effect of geometry on fracture toughness of steel in the ductile-to-brittle transition region has been studied. The critical crack-tip opening displacement (CTOD) and the critical J-integral have been measured as a function of temperature for ten fracture specimen configurations of ABS grade EH36 steel. In addition, two specimen geometries of commercially pure iron were tested. Thicker specimens tend to promote a higher degree of triaxiality near the crack tip, thereby elevating the flow'stress and making it easier to reach the cleavage stress. This causes a shift in the ductile-to-brittle transition to higher temperatures. Decreasing the uncracked ligament length of bend specimens also tends to shift the transition to higher temperatures. Changing the loading conditions from bending to tension tends to shift the transition to lower temperatures when the ligament is relatively short. When the ligament length is large the transitionregion fracture behavior of a bend specimen approaches that of a tension specimen with a similar geometry.

A technique was developed to experimentally measure crack-tip constraint. Constraint decreased with crack blunting. This relaxation in constraint was modeled by a simple spring analog. The model was used to predict ductile-to-brittle transition curves for hypothetical structures. Some of the applications and limitations of the model are discussed.

Key words: crack-tip opening displacement; crack-tip constraint; ductile-to-brittle; transition; elastic-plastic fracture; J-integral; mechanical properties; structural steels; toughness. 
Effect of Crack-Tip Region Constraint on Fracture in the Ductile-to-Brittle Transition

\section{T. L. Anderson}

\section{INTRODUCTION}

Fracture mechanics is a fairly new branch of materials science which seeks to quantify the critical combinations of stress and crack size for crack extension. There are two main ideas in fracture mechanics. First, fracture occurs when the driving force for fracture, a function of stress and flaw size, exceeds the material's resistance to crack extension referred to as the fracture toughness. Second, fracture toughness is a geometry-independent material property; i.e., a simple laboratory specimen and a large structure both fracture at the same critical value of driving force. Both of these ideas work quite well for materials that behave in a linear elastic manner; i.e., fracture occurs prior to extensive plastic deformation. Recent progress in ductile fracture mechanics suggests that these ideas are also valid for materials that fracture in a fully ductile manner [1].

Contrary to common assumptions, there is not a geometry-independent fracture criterion for the transition region between linear elastic and fully-ductile fracture. The fracture toughness of steels which undergo a ductile-to-brittle transition is dependent on geometry in the transition region. Thus, one of the main ideas of fracture mechanics, the applicability of laboratory fracture toughness data to practical structures, is not valid.

This document summarizes the author's efforts to characterize the geometry effect on fracture toughness in the transition region. Basic concepts of fracture mechanics as well as recent research pertinent to 
this study are summarized below. Following the literature review the statement of the problem objectives, and technical approach are given. The experimental procedures, the results and discussion, and the summary and conclusions are given in Chapters 2, 3, and 4, respectively. New developments and refinements of fracture toughness test methods which resulted from this investigation are included as an appendix.

\subsection{Linear Elastic Fracture Mechanics*}

The physical basis of fracture mechanics stems from the work of Griffith [3] who demonstrated that the strain energy released upon crack extension is the driving force for fracture. The strain energy, $U$, is the work done by load, $P$, causing a displacement, $\Delta$ :

$$
U=\frac{P \Delta}{2}=\frac{C P^{2}}{2}
$$

where $C=\frac{\Delta}{P}=$ elastic compliance.

The loss of elastic energy upon crack extension of unit area, A, is defined as the strain energy release rate, $G$ :

$$
G=-\left.\frac{d U}{d A}\right|_{\Delta}=\frac{P^{2}}{2} \frac{d C}{d A}=\left.\frac{d U}{d A}\right|_{P}
$$

*Sections 1.1 and 1.2 are taken from Ref. [2]. 
Irwin and Kies [4] used this relationship to determine the fracture resistance of structural materials, $G_{C}$, by measuring the critical load, $\mathrm{P}_{\mathrm{C}}$, in a specimen with a known compliance function, $\mathrm{dC} / \mathrm{dA}$. .

\subsubsection{The Stress Intensity Factor}

Irwin [5], who determined the stress distribution near the tip of a crack located in a linear elastic body, developed a stress analysis basis for fracture mechanics. The magnitude of the crack tip stress field, $\sigma_{i j}$, was found to be proportional to a single parameter, $k$, the stress intensity factor:

$$
\sigma_{i j}=\frac{K}{\sqrt{2 \pi r}} f_{i j}(\theta)=K \cdot f(\text { position })
$$

where, $r$ and $\theta$ are cylindrical position coordinates; $r=0$ at the crack tip and $\theta=0$ in the crack plane. $K$ is a function of the applied stress, $\sigma$, crack length, a, and a factor dependent on structural geometry, $Y(a)$ :

$$
K=Y(a) \sigma \sqrt{a} \text {. }
$$

Tada, Paris and Irwin [6] and Sih [7] have published handbooks of $K$ formulae for various geometries and boundary conditions.

Irwin [5] demonstrated that the strain energy release rate and stress intensity factor are related:

$$
K^{2}=E^{\prime} G
$$


where, for plane stress, $E^{\prime}=E$, the elastic modulus; and for plane strain, $E^{\prime}=E /\left(1-\nu^{2}\right)$, where $\nu$ is Poisson's ratio. Thus, it is equivalent to attributing the driving force for fracture to the crack tip stress field, which is proportional to $K$, or to the elastic strain energy release rate, $G$. The stress intensity, $K$, is used more commonly than $G$ because $K$ can be computed for different structural geometries using stress analysis techniques.

\subsubsection{Fracture Toughness}

Fracture occurs when the crack tip stress field reaches a critical magnitude, i.e., when $k$ reaches $K_{c}$, the fracture toughness of the material. $K_{c}$ is a mechanical property that is a function of temperature, loading rate and microstructure, much the same as yield strength is; however, $K_{c}$ is also a function of the extent of crack tip plasticity relative to the other specimen (or structural) dimensions. If the plasticity is small compared to the specimen dimensions and the crack size, then $K_{C}$ approaches a constant minimum value defined as $K_{I c}$, the plane strain fracture toughness.

Measurements of fracture toughness based on linear elastic theory are limited to the case of plane strain testing in accordance with ASTM Standard E 399.

$$
B, a, w-a \geq 2.5\left(\frac{K_{I c}}{\sigma_{y s}}\right)^{2}
$$


where $B, a$, and $W$ are defined in Figure 1. The specimens are precracked by fatigue cycling to an initial crack length of $a / W=0.5$ and subsequently loaded to failure. $K_{\text {Ic }}$ is calculated from the critical load, $P_{Q}$, the measured crack length, the specimen dimensions and the specimen calibration function, $Y\left(\frac{a}{W}\right)$, as follows:

$$
K_{Q}=\frac{P Q}{B \sqrt{W}} Y\left(\frac{a}{W}\right) \text {. }
$$

$K_{\text {Ic }}=K_{Q}$ if all the conditions of ASTM E 399 are met, i.e., precracking procedures, load-displacement record, specimen dimensions, etc.

\subsubsection{Crack Tip Plasticity}

Applicability of the linear elastic analysis has been extended to conditions approaching net-section yielding by correcting for the zone of plasticity that exists at the crack tip [8]. The idea is that the plastic material at the crack tip strains without carrying the incremental load; therefore, in the elastic sense, the crack behaves as if it were slightly longer. The adjustment is made by adding the radius of the plastic zone, $r_{y}$, to the apparent crack length:

$$
\begin{aligned}
& K=Y\left(a+r_{y}\right) \sigma \sqrt{a+r_{y}} \\
& r_{y}=\frac{1}{2 \pi}\left(\frac{K}{\sigma_{Y}}\right)^{2}
\end{aligned}
$$

where, $\sigma_{Y}$ is the yield strength at the crack tip. The $r_{y}$ correction modifies the crack tip stress field to account for the elastic stress redistribution due to the localized plasticity. 


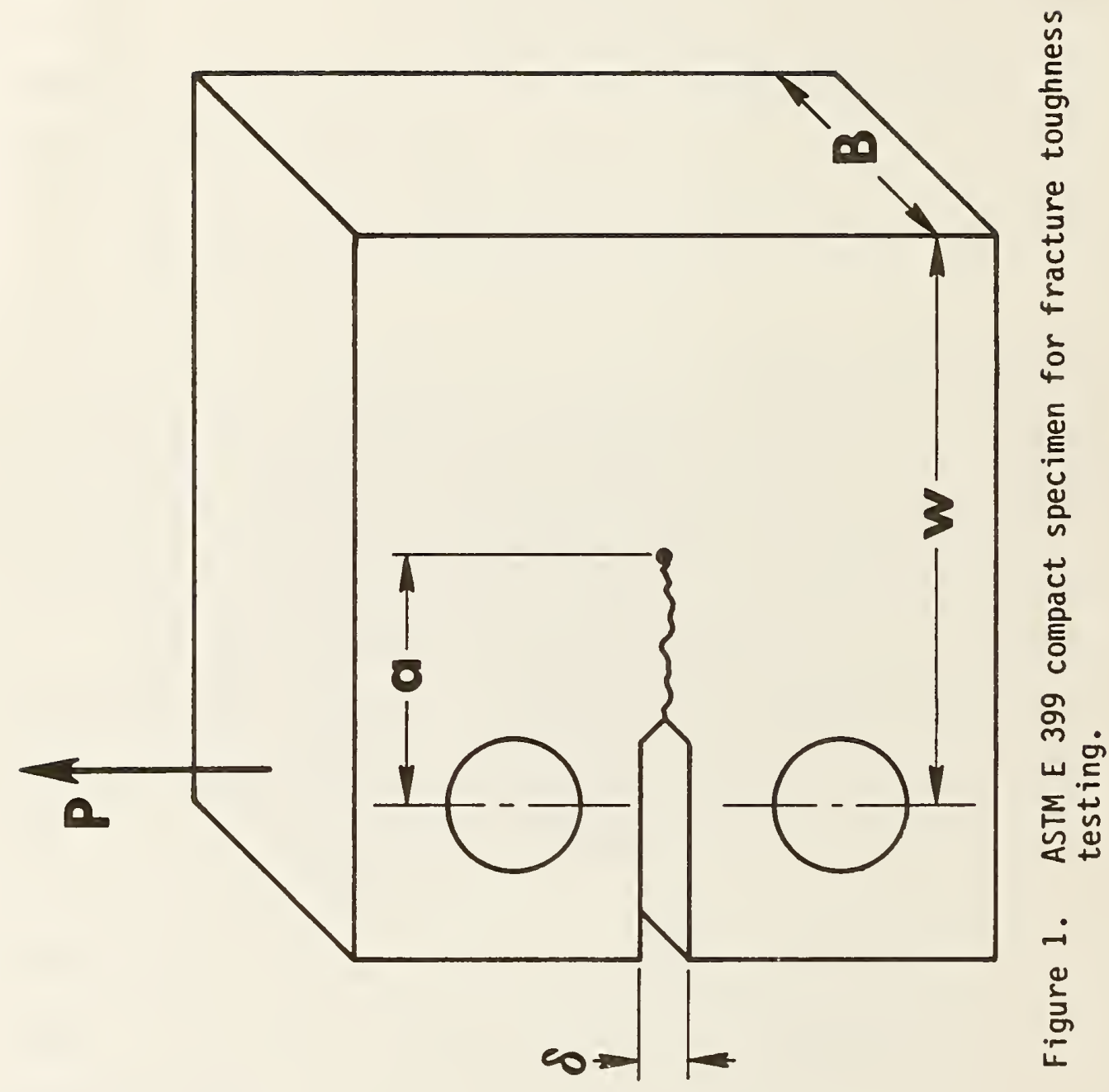


The extent of crack tip plasticity is influenced by specimen dimensions. This is particularly true for thickness. As specimen thickness increases, $\sigma_{\gamma}$ increases from $\sigma_{y s}$ to $\sqrt{3} \sigma_{y s}$ due to through-thethickness elastic constraint [9]. The maximum value of $\sigma_{y}$ is reached when the plastic zone size is limited to about $5 \%$ of the thickness. Thus, in a given material, the plastic zone size as computed by equation (9) can vary with thickness by a factor of three -- leading to a strong dependence of $K_{c}$ on thickness.

\subsubsection{Summary Comments}

The crack tip stress field is the driving force for fracture and the magnitude of this stress field is proportional to the stress intensity factor, $K . \quad K$ is a function of crack size, applied stress and structural geometry and can be computed using stress analysis methods. The resistance to fracture is a material property defined as the fracture toughness, $K_{c}$; fracture occurs when $K=K_{c}$. Thus, for a given structural geometry and material, critical crack sizes can be determined as a function of applied stress and vice-versa:

$$
a_{c}=\left[\frac{K_{c}}{\sigma Y(a)}\right]^{2} \text {. }
$$

The approach is applicable to conditions of localized crack tip plasticity, i.e., where $\sigma \leq .8 \sigma_{y s}$ or $r_{y} \leq 0.3 a$, when the $r_{y}$ correction is used to account for crack tip plasticity [10]. 


\subsection{Elastic-Plastic Fracture Mechanics}

Many of the high toughness structural materials undergo extensive plastic deformation prior to fracture. Thus, the concepts of linear elastic fracture mechanics must be extended to account for elasticplastic behavior. The two approaches of interest in the present investigation are the crack-tip opening displacement (CTOD) and the $J$ integral. 1.2.1 The Crack-Tip Opening Displacement

The CTOD concept is a crack tip strain criterion for fracture stemming primarily from the work of Wells $[11,12]$. Wells' hypothes is was that the opening of the crack faces was strictly related to the crack extension force, $G$, and the stress intensity factor, $K$, under locally plastic conditions. Wells used an energy balance argument to derive an expression for CTOD [12]:

$$
\delta=\frac{K_{I}^{2}}{E \sigma_{y}}=\frac{G_{I}}{\sigma_{y}}
$$

where $\delta$ is the CTOD.

In 1965 Burdekin and Stone [13] illustrated how the CTOD concept could be used to extend the capability of conventional fracture mechanics to the elastic-plastic case. A Dugdale strip-yield model [14] was used to develop the following equation for plane stress Mode I crack-tip displacements under monotonic loading.

$$
\delta=\frac{\sigma a}{\pi E} \ln \sec \left(\frac{\pi}{2} \frac{\sigma}{\sigma_{y}}\right)
$$

A fracture toughness test method based on the CTOD concept was developed by investigations at the Welding Institute [15-17]. The CTOD 
test specimen (Fig. 2) contained a fatigue-precracked notch and was loaded in three-point bending to fracture. The critical CTOD was obtained from the load versus clip-gage displacement record. The clip-gage displacement, $V$, was measured across the notch mouth as shown in Figure 2. The $V$ was converted to CTOD using the following relationship [15].

$$
\delta=\frac{v}{1+\left(\frac{a+z}{r_{0}(W-a)}\right.}
$$

where $z=$ knife edge thickness

$$
\begin{aligned}
& a=\text { crack length } \\
& W=\text { specimen width } \\
& r_{0}=\text { rotational factor } .
\end{aligned}
$$

This equation was derived assuming tinat the crack faces opened by a simple hinge mechanism about a center of rotation. The value of $r_{0}$ was assumed to be 0.5 in early work.

The current British standard for CTOD testing [18] specifies that the CTOD be calculated from the following relationship.

$$
\delta=\frac{K^{2}}{2 \sigma_{y} E^{\prime}}+\frac{0.4(W-a) V_{p}}{0.4 W+0.6 a+z}
$$

where the first term is the elastic component of CTOD, $\delta$, and the second term is the plastic component, $\delta_{p}$. The plastic component of the clip-gage displacement, $V_{p}$, is estimated by constructing a line, parallel to the elastic-loading line, from the critical point on the load- 


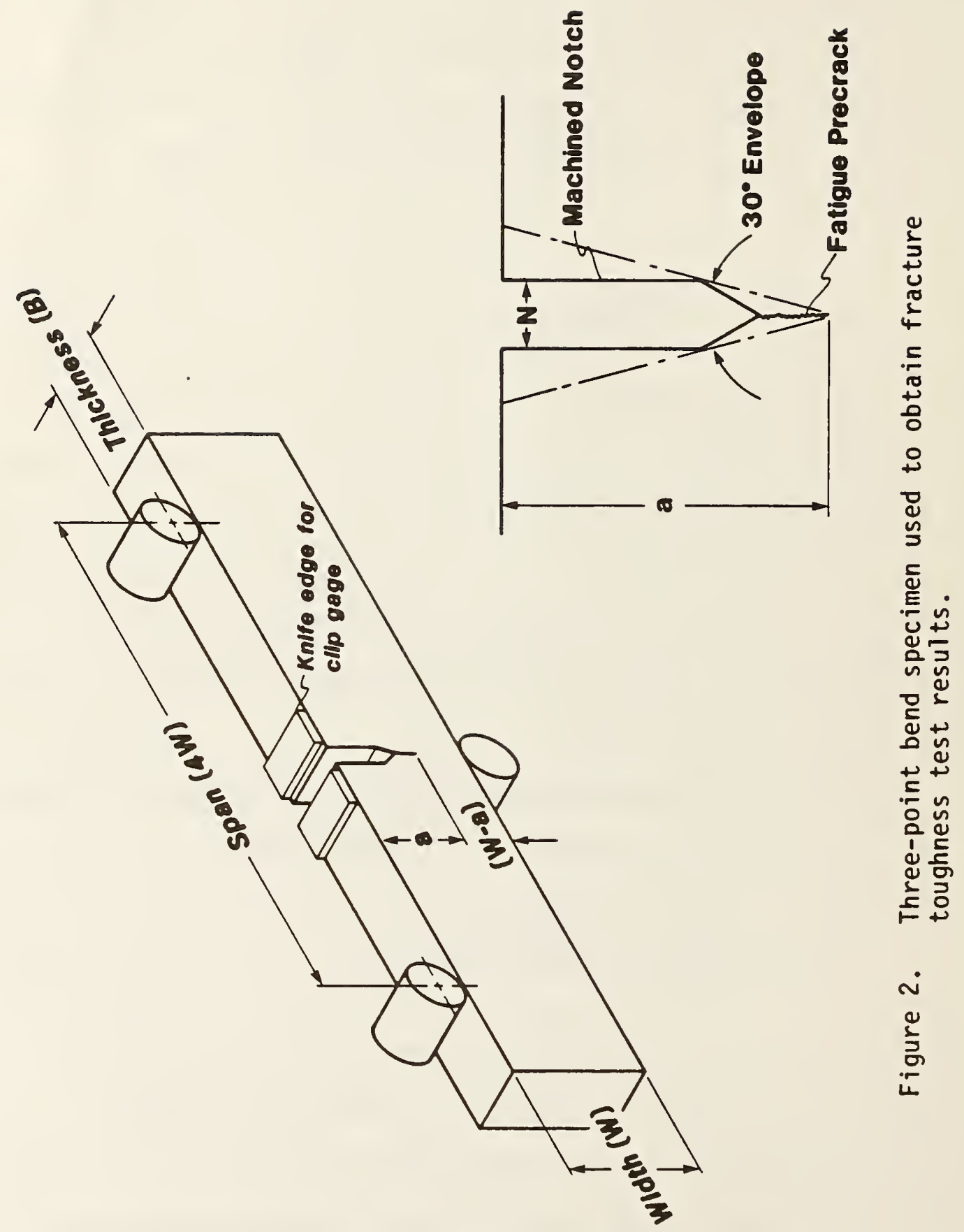


displacement curve to zero load. The elastic component of the clip gage displacement is then subtracted from the total displacement. The stress intensity factor for the elastic CTOD calculation is obtained from the following relationship.

$$
K=\frac{Y P}{B W^{1 / 2}}
$$

where $P$ is the applied load and $Y$ is the stress intensity coefficient. $Y$ is a function of specimen geometry and can be obtained for a given crack length-to-width ratio (a/W) from the test standard [18].

The type of critical CTOD reported depends on the nature of the observed fracture event. This is illustrated schematically in Figure 3 for a steel which undergoes a ductile-to-brittle transition. At low temperatures the steel fails by cleavage and $\delta_{C}$ is measured experimentally. As the test temperature increases cleavage becomes less favorable and the fracture toughness increases. Eventually the fracture mode changes to microvoid coalescence and the crack grows in a stable manner. $\delta_{j}$ is defined as the value of CTOD at the onset of tearing. At temperatures slightly above the fracture mode change, stable tearing can be followed by unstable cleavage. When this occurs, $\delta_{u}$ is measured at the instability point. On the upper shelf of toughness, the steel reaches a point of plastic collapse when the work-hardening cannot keep pace with the decrease in ligament area caused by stable crack growth. $\delta_{m}$ is then measured at the point of maximum load in a bend test. $\delta_{R}$ is the total CTOD, i.e. $\delta_{i}$ plus the portion of CTOD associated with tearing. A plot 

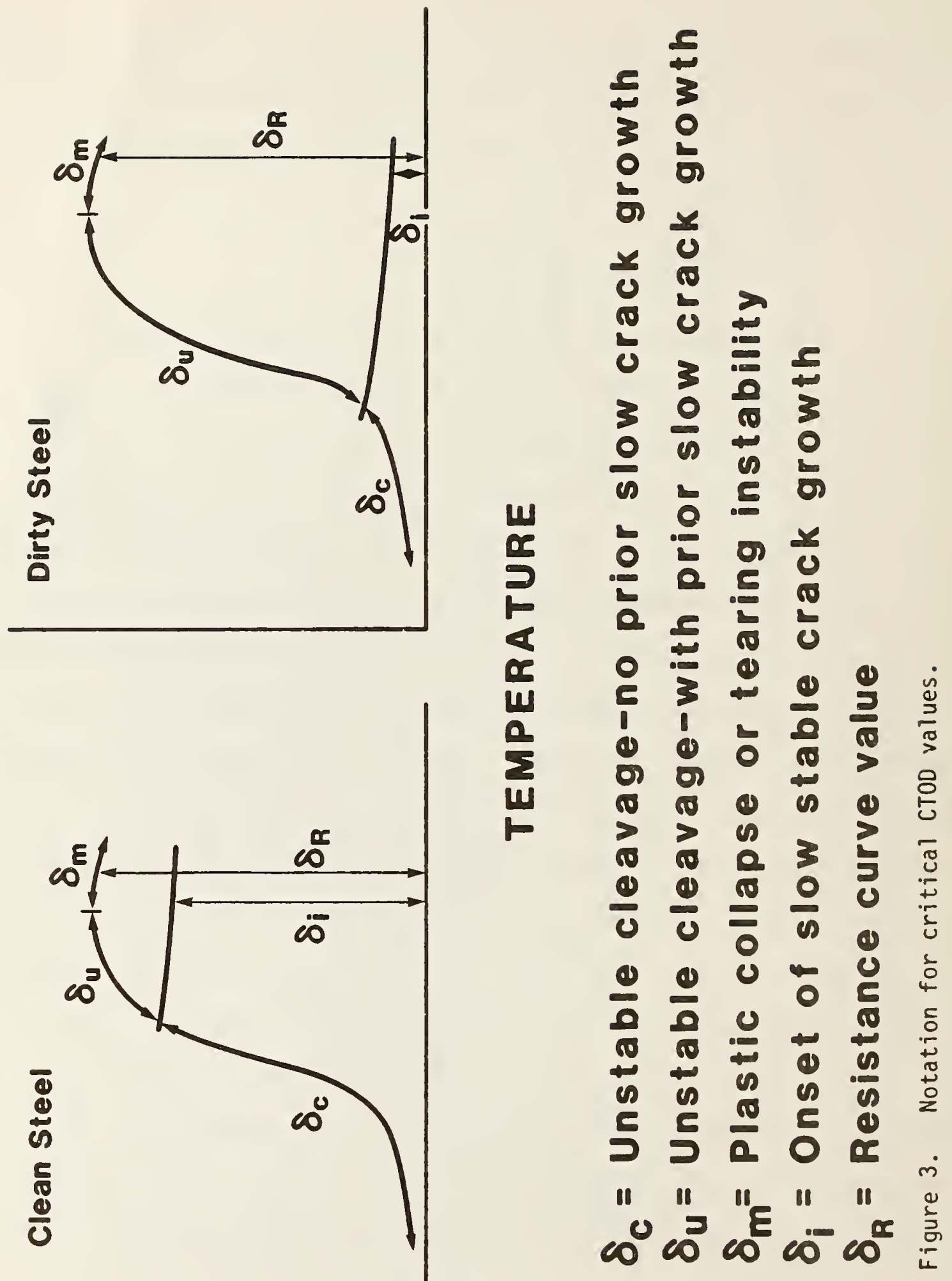

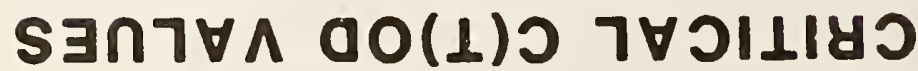


of $\delta_{R}$ versus $\Delta a$ is the resistance $(R)$ curve, a measure of a material's resistance to crack growth. Figure 3 also shows that $\delta_{i}$ is not a strong function of temperature, but it is a function of the inclusion content.

\subsubsection{J-Integral}

The J-integral is a characterization developed by Rice [19] of the elastic-plastic field in the vicinity of the crack tip. The use of the J-integral as a fracture criterion was suggested and experimentally demonstrated by Begley and Landes [20]. $J$ is defined as the 1 ine integral :

$$
J=\delta_{\Gamma}\left[w d y-\neq\left(\frac{\partial \vec{u}}{\partial x}\right) d s\right\rfloor
$$

where, $\Gamma$ is any contour surrounding the crack tip,

$$
\begin{aligned}
& w \text { is the strain-energy density, } \\
& \neq \text { is the stress vector normal to } \Gamma \text {, } \\
& \vec{u} \text { is the displacement vector, and } \\
& s \text { is the arc length along } \Gamma \text {. }
\end{aligned}
$$

The J-integral is path independent for linear and non-linear elastic materials [19] and nearly so for most structural materials under monotonic loading conditions [21]. Thus, $\mathrm{J}$ can be computed using numerical methods by analyzing along a contour away from the crack tip, i.e., in a region where the analysis methods are quite accurate.

An equivalent interpretation is that $\mathrm{J}$ is equal to the change of potential energy $U$, upon an increment of crack extension $A$ :

$$
J=-\frac{d U}{d A} \text {. }
$$


For the linear elastic case the potential energy equals the strain energy and, therefore, equation (17) is the same as equation (2) and $J=G$. Thus, $J$ appears to be a logical extension of linear elastic fracture mechanics into the elastic-plastic range. Due to the irreversibility of plastic deformation, the energy interpretation of the J-integral does not apply to the process of crack extension in elasticplastic materials -- as $G$ is for elastic materials. $J$ is simply an analytically convenient, measurable parameter that is a characteristic of the elastic-plastic field at the crack tip. The contribution of Begley and Landes [20] was to demonstrate that crack initiation under elastic-plastic conditions occurs at a characteristic value of $\mathrm{J}$, called $\mathrm{J}_{\mathrm{IC}}$, that is related to $\mathrm{K}_{\mathrm{Ic}}$ in the same way $G$ is related to $K$ in equation (5). Thus, $J$ integral methods can be used to determine $K_{I c}$ in specimens significantly smaller than the size requirements of equation (6).

Methods of measuring $\mathrm{J}_{\mathrm{IC}}$ are covered by ASTM Standard E 813. A deeply notched specimen of the compact tension or single-edge notch bend design is precracked to $a / W \cong 0.6$. The specimen is loaded incrementally to a series of J-levels. After each increment of loading, $J$ and the crack extension, $\Delta \mathrm{a}$, are measured. The load-displacement curve is recorded on an $X-Y$ plotter. Displacement is measured along the load line. $J$ is calculated from the load-displacement record and specimen dimensions using:

$$
J=\frac{n U}{b B},
$$


where $U$ is the area under the load-displacement record, $b$ is the ligament length, $(W-a)$, and $n$ is a dimensionless parameter dependent on specimen geometry. $\Delta a$ is measured by either the crack marking technique or the unloading compliance technique described in ASTM E 813. The results of a test series are plotted as $J$ versus $\Delta a$, and the $J_{I c}$ is defined as the extrapolation of the best-fit curve to the blunting line defined by $J=2 \sigma_{y} \Delta a$. $K_{I c}$ is related to $J_{I c}$ as follows:

$$
K_{I C}(J)=\sqrt{E^{\prime} J_{I C}}
$$

The $J_{\text {Ic }}$ test method is limited to measurement of the onset of ductile tearing. Thus, it is not applicable to the measurement of fracture toughness when cleavage is the micromode of fracture. Recently Dawes [23] has proposed the adoption of a critical $J$ notation equivalent to the CTOD notation of Figure 3 . This notation, shown in Figure 4 , would make the $\mathrm{J}$ concept applicable to all micromechanisms of fracture. This proposed notation is used in the present study.

\subsubsection{Summary Comments}

The CTOD and J-integral concepts are efforts to define single parameter characterizations of the fracture process that are applicable to linear elastic and general yielding fracture. In the CTOD concept, attention is focused at the crack tip; fracture theoretically occurs when a critical displacement develops at the crack tip. The J-integral 

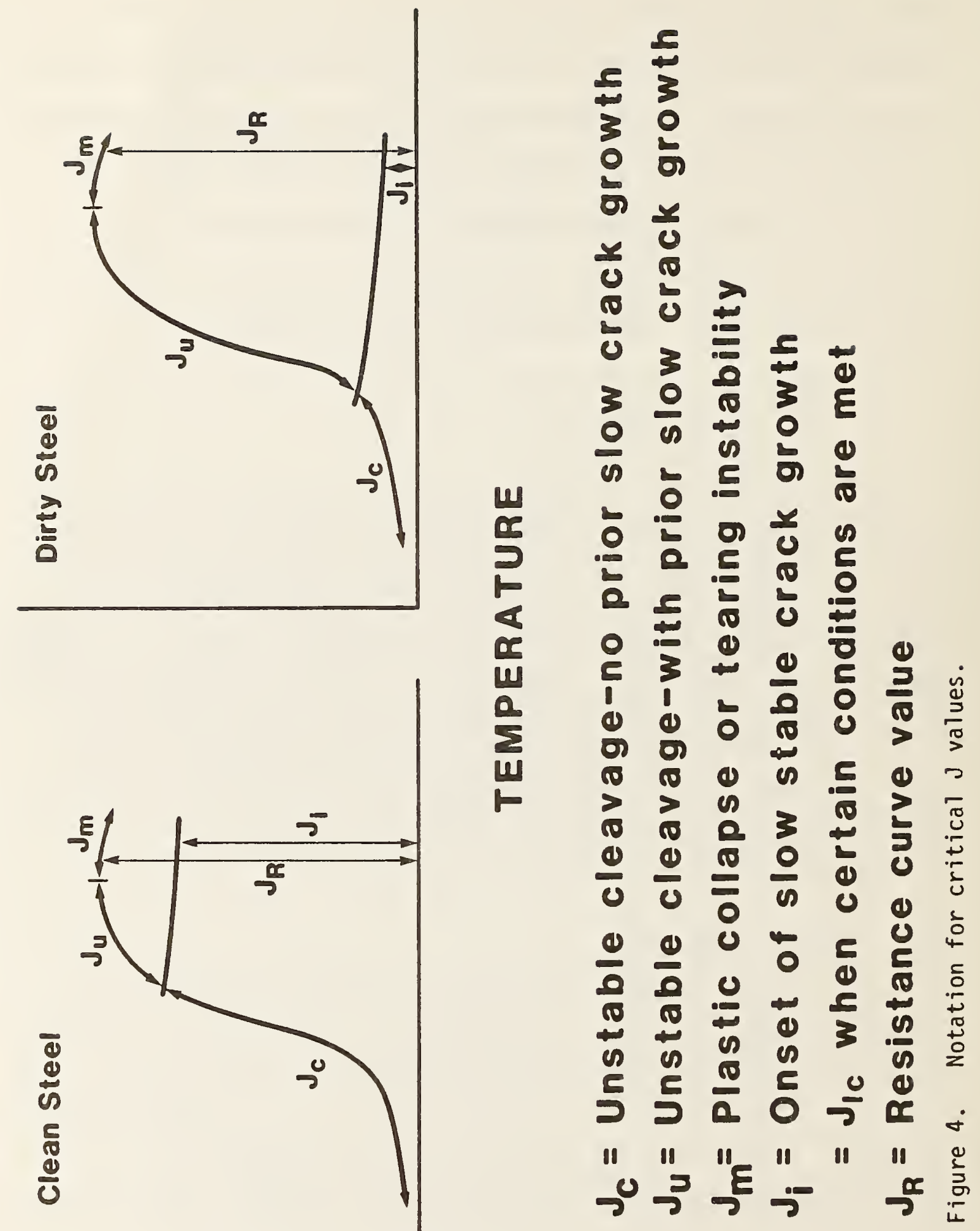

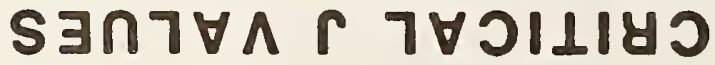


concept examines the stress-strain conditions along an arbitrary contour away from the crack tip; fracture theoretically occurs when the potential energy available for crack extension reaches a critical value defined by $J$. For the linear elastic case, $\delta_{C}$ and $J_{I C}$ are consistent with the linear elastic fracture criteria:

$$
G_{I C}=\frac{K_{I C}^{2}}{E^{\prime}}=J_{I C}=2 \sigma_{Y} \delta_{C} .
$$

There has been a great deal of research in recent years on ductile fracture. Models have been developed to predict fracture by tearing instability using $\mathrm{J}$-integral R-curves and to predict fracture by plastic collapse using limit-load expressions [22]. This work is not reviewed here because it is not directly relevant to fracture of structural steels at low temperatures.

\subsection{Micromechanisms of Crack Extension}

Fracture usually occurs by one of three mechanisms as illustrated in Figure 5. Cleavage is rapid, unstable fracture associated with brittle materials, while ductile tearing (or microvoid coalescence) can occur in a slow, stable manner. Intergranular cracking can occur in either a ductile or brittle manner. It is usually associated with a corrosive environment and/or grain-boundary segregation. Since intergranular cracking did not occur in this investigation, only cleavage and microvoid coalescence are reviewed in this section. 


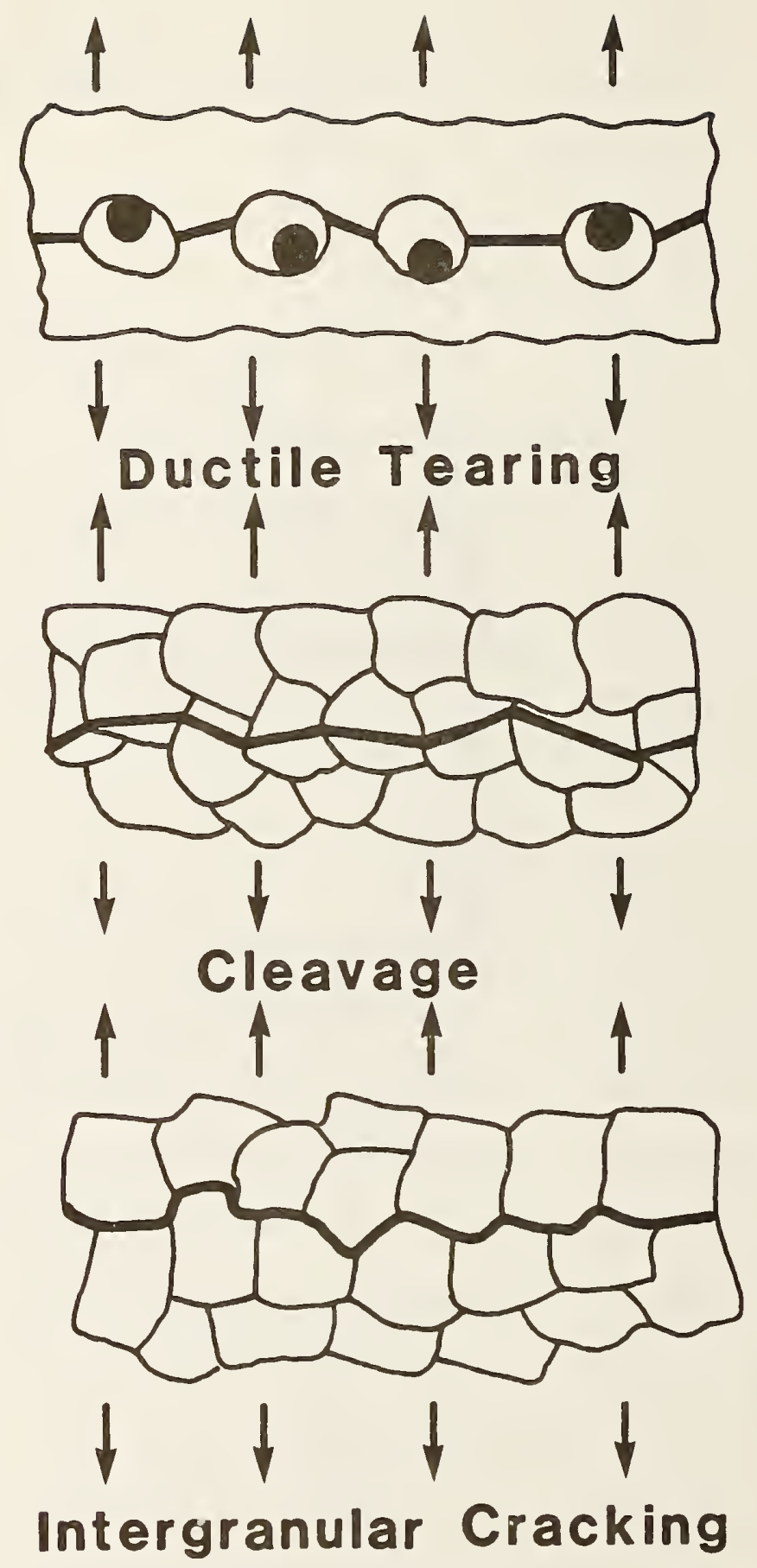

Figure 5. The three basic micromechanisms of fracture. 
Local plastic flow around the crack tip precedes fracture in both ductile and brittle materials. For cleavage the plastic zone must increase in size until sufficient local stress has been generated to propagate a crack nucleus formed in some microstructural feature. For microvoid coalescence or ductile tearing, a critical strain must be reached for the coalescence of voids formed around second phase particles. Fracture toughness parameters will necessarily differ for different fracture mechanisms [23].

\subsubsection{Cleavage}

Plastic flow is necessary to propagate crack nuclei but cracks may initiate and propagate at very low macroscopic strains because the initial flow is localized at a stress concentration [24]. Generally, a material's susceptability to cleavage is promoted by any factor which increases the yield strength such as low temperature, constraint which produces triaxiality, radiation damage, prestrain and strain aging. Refinement of grain size increases the yield strength but improves a steel's resistance to cleavage fracture because grain-size refinement also increases the fracture stress [24-27].

Cleavage is nucleated when a critical value of "effective shear stress" is attained corresponding to newly created slip dislocations which fracture carbides, either by the stress induced at the end of a pile-up [28-30] or by plastically straining the matrix beyond the fracture strain of the particles. For normalized or furnace cooled 
steels the effective shear stress is independent of temperature until such a low temperature is attained that plastic deformation occurs by twinning rather than slip [24].

The Petch-Hall equation can be written in terms of shear stress:

$$
\tau_{y}=\tau_{i}+k_{y} d^{-1 / 2}
$$

where $\tau_{y}$ is the shear yield stress, $\tau_{j}$ is the friction stress and $d$ is the grain diameter. The friction stress, $\tau_{j}$, increases rapidly with decreasing temperature while the effective shear stress, $k_{y} d^{-1 / 2}$, is relatively constant with temperature. At low temperatures a crack nucleus can propagate when the plastic zone is small because the yield stress is high and little elevation by triaxiality and strain hardening is needed to satisfy critical conditions for propagation. At higher temperatures the yield stress is smaller so a larger plastic zone is required to produce the necessary local stress.

Cleavage fracture is propagation controlled and depends on the level of the local tensile stress. Values of the critical stress, $\sigma_{f}$, are only slightly temperature dependent [24].

Models of propagation-controlled, slip-initiated cleavage fracture were proposed by Knott [31], Cottrel1 [34], and McMahon and Cohen [30]. The Knott and Cottrell models treat cleavage as the propagation of a nucleus formed by the interaction of slip dislocations. The McMahon and Cohen model considers cleavage as the propagation of a small crack in a grain boundary carbide. A model by Smith [35] which takes into account 
both the effect of carbide thickness and dislocation interactions gives the following expression for $\sigma_{f}$.

$$
\left(\frac{C_{0}}{d}\right) \sigma_{f}{ }^{2}+\tau_{\text {eff }}^{2}\left[\frac{1}{2}+\frac{2}{\pi}\left(\frac{C_{0}}{d}\right)^{1 / 2} \frac{\left.\tau_{i}\right]^{2}}{\tau_{\text {eff }}} \geq \frac{4 E_{\gamma_{p}}}{\pi\left(7-v^{2}\right) d}\right.
$$

where $C_{0}$ is the thickness of a grain boundary carbide,

$$
\tau_{\text {eff }}=k_{y} d^{-\frac{1}{2}}
$$

is the effective shear stress, and $\gamma_{p}$ is a surface energy or plastic work term. The critical event is taken to be the propagation of a crack, nucleus formed in a grain boundary carbide into the adjacent ferrite matrix.

Knott [24] compiled the results of several authors who measured $\sigma_{f}$ in a variety of mild steels as a function of the reciprocal square root of grain size. These data are shown in Figure 6 . The fracture strength obviously increases rapidly with $d^{-\frac{1}{2}}$ al though the scatter is great at high $d^{-\frac{1}{2}}$ values. The data in Figure 6 were taken at various temperatures but the effect of temperature on $\sigma_{f}$ is small. Data of Curry and Knott [25] which were taken at a constant temperature $\left(-120^{\circ} \mathrm{C}\right)$ follow the common trend.

A problem arises if one attempts to use equation (22) to explain the grain size dependence of $\sigma_{f}$. If $k_{y} d^{-\frac{1}{2}}$ is substituted for $\tau_{\text {eff }}$ in equation (22) the grain size terms cancel, leaving a relationship whose only microstructural parameter is the carbide width, $C_{0}$. Knott [24] suggested that a possible reason for this anomaly is that the ratio of $\mathrm{C}_{0} / \mathrm{d}$ remains constant upon cooling from the austenitic range, since both 


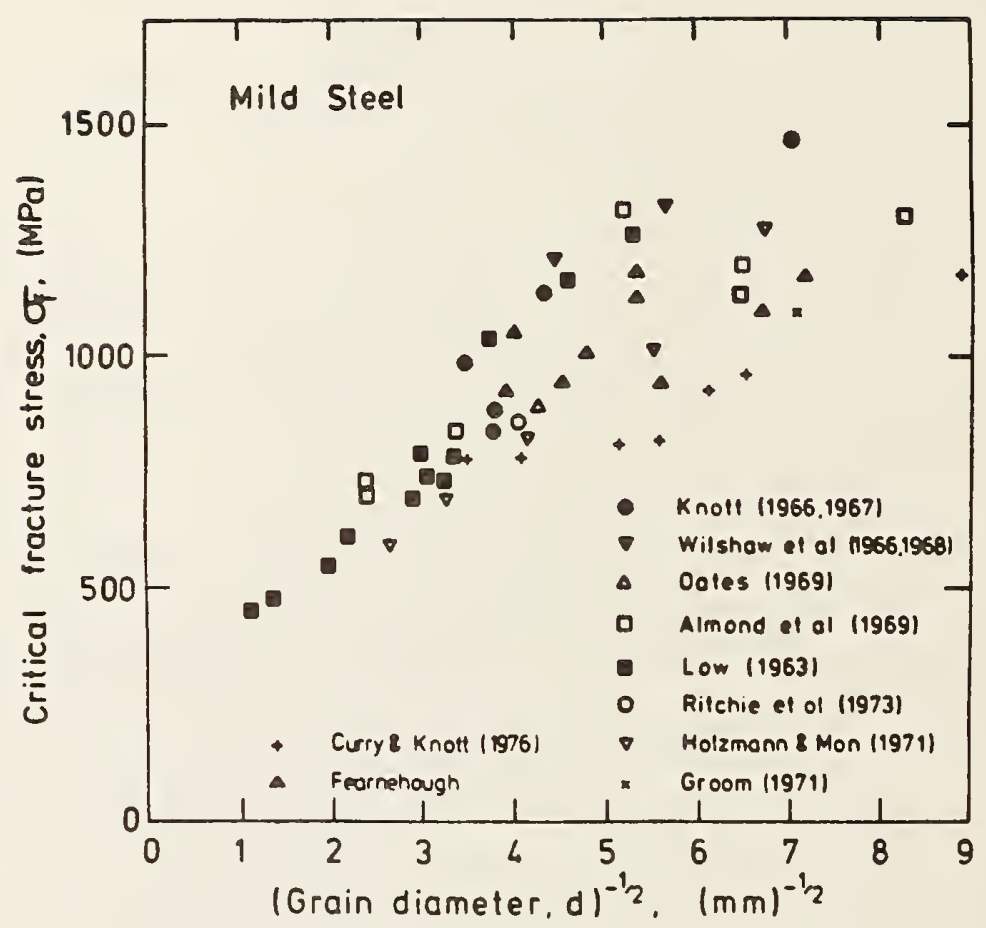

Figure 6. Variation of critical fracture stress with grain size; compiled by Knott [24]. 
$C_{0}$ and $d$ depend on similar diffusion phenomena. Experimental data of Curry and Knott [25] show that there is a strong relationship between the two parameters, although it is not quite linear.

The relative effect of dislocation pile-up on propagation of a cleavage crack is a function of $c_{0} / d[35]$. A crack in a thick carbide particle is large enough to be treated as a Griffith crack and the stresses from local dislocations are of secondary importance. However, for thin particles the crack nucleus does not extend far from the tip of the pile-up and the dislocations have an important effect.

Knott [24] has modified the model for low- and medium-carbon steels with spherical carbides. The critical event is taken to be the propagation of a nucleus in the spheroidal carbide into the ferrite matrix. The dislocation contribution is ignored because pile-ups do not occur. Instead the dislocations tangle around the carbide and form a cell structure. The size of the cell structure depends on $x_{0}$, where $x_{0}$ is the interparticle spacing, rather than on $d$. The expression for the local fracture stress is given by

$$
\sigma_{f}=\left(\frac{\pi E_{p}}{2 C o}\right)^{\frac{1}{2}}
$$

where $C_{0}$ is the carbide diameter. Values of $\gamma_{p}$ (for ferrite) inferred from equation (23) and experimental data are around $14 \mathrm{~J} \cdot \mathrm{m}^{-2}[24]$. This is consistent with values of $\gamma_{p}$ obtained for mild steels from equation (22).

Investigations of the cleavage fracture of low-carbon bainite and martensite have established that the microstructural feature controlling 
crack propagation is the packet size [36]. Since large misorientations often occur at packet boundaries the direction of propagation must change when the crack encounters a packet boundary. Extra work must be done to allow a crack in a favorably oriented packet to propagate into the adjacent packet. This is considered the critical event and a fracture criterion based on packet size has been developed [36]:

$$
\sigma_{f}=\frac{4 E \gamma_{p}}{\pi\left(1-v^{2}\right) d_{p}}
$$

here $d_{p}$ is the packet diameter. The inferred $\gamma_{p}$ value is $120 \mathrm{~J} \cdot \mathrm{m}^{-2}$ as compared to $14 \mathrm{~J} \cdot \mathrm{m}^{-2}$ for ferrite.

It has long been recognized that a critical fracture stress must be exceeded at a crack tip before the crack can propagate by cleavage [24-37]. It has been discovered, however, that while this is a necessary condition it is not sufficient. It is now believed that the fracture stress must be exceeded over a critical distance which is characteristic of a given microstructure. This concept is illustrated in Figure 7. Ritchie, et. al. [38] applied this concept to develop a model which predicts temperature variations of $\mathrm{K}_{\mathrm{Ic}}$ in mild steel. Figure 8 shows experimental data fitted to their model. Predictions of $\mathrm{K}_{\mathrm{Ic}}$ were made using an experimental value for $\sigma_{f}$, which was found to be insensitive to temperature variations in the range studied, and various assumed values for critical distance. Figure 8 shows that excellent agreement was attained by assuming a critical distance of 2 grain diameters. 


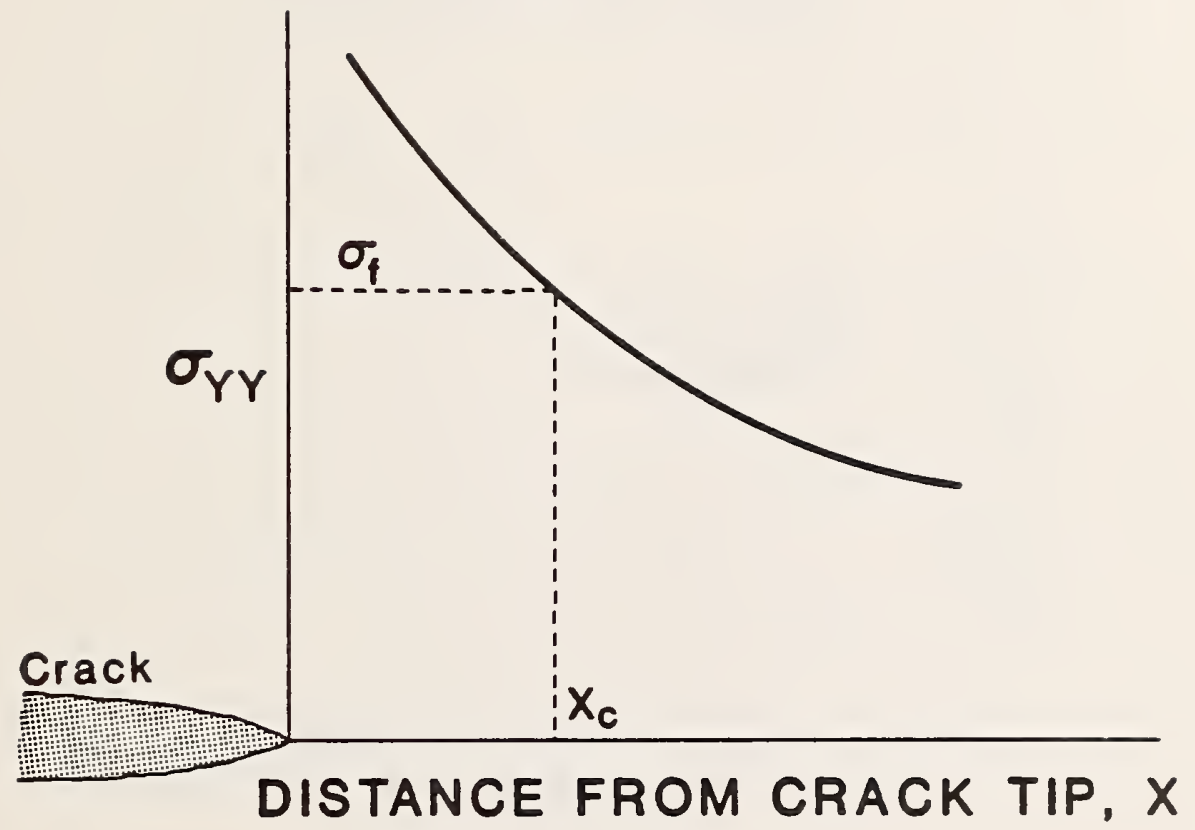

Figure 7. The Ritchie-Knott-Rice model for cleavage fracture [38]. Fracture occurs when the fracture stress is exceeded over a critical distance ahead of the crack tip. 
- Predicted from of asuming $d_{c}$ - one groin diameter

- Predicted ossuming $d_{c}=$ two groin diometers

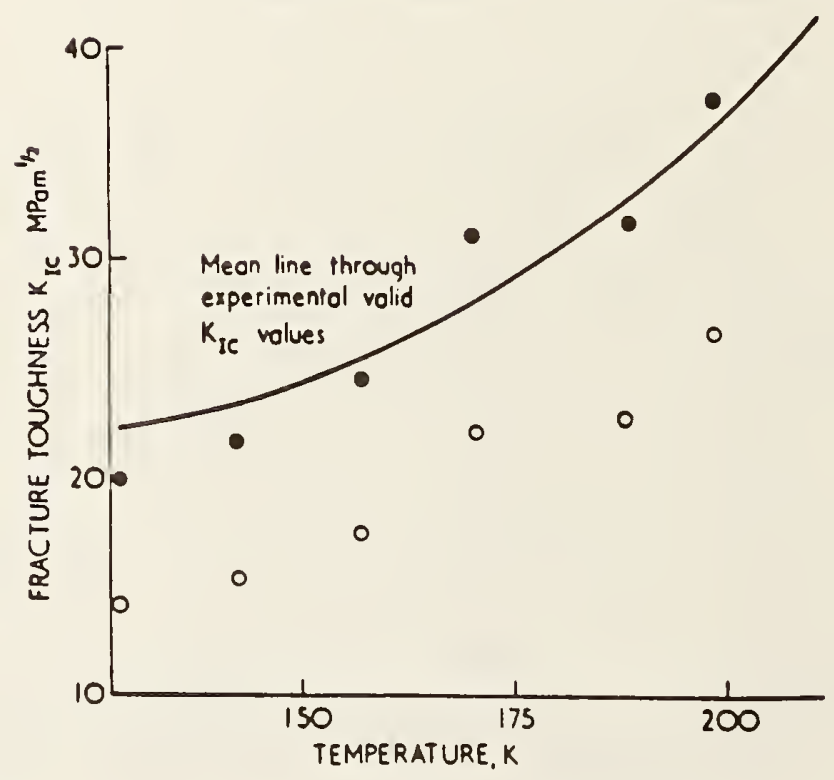
Figure 8. Comparison of predicted and experimental $\mathrm{K}_{\mathrm{IC}}$ values
$[38]$. 
The effect of grain size on critical distance has been studied by Curry and Knott [25]. For their mild steel the critical distance was about four times the grain diameter for $d$ above about $50 \mu \mathrm{m}$, but was relatively independent of grain size for finer grain sizes.

The apparent reason for this is that carbides of sufficient thickness to satisfy the propagation conditions of equation (22) are more abundant in coarse grains. In the case of finer grains, more grains have to be sampled to find a sufficiently thick carbide, and the critical distance increases with respect to grain size.

Curry and Knott [29] proposed a model for predicting fracture toughness on the basis of finding favorably oriented carbide particles within the plastic zone. According to this model, crack nucleus size distribution is some multiple of the carbide particle size distribution. The probability of finding a crack nucleus subjected to a stress greater than or equal to $\sigma_{f}$ is estimated for each nucleus radius and is summed over all nucleus radii. Cleavage occurs when this probability equals unity.

Curry [39], in a separate publication, argued that the model proposed by Ritchie, et. al. [38] is compatible with the statistically based model of Curry and Knott [29]. The characteristic distance can be related to the mean carbide radius or thickness.

Evans and Hutchinson [40] have also developed a statistically based model for cleavage fracture. This model provides a rationale for the critical distance. According to the model the critical distance, which 
depends on the size distribution and volume concentration of grain boundary carbides, is the distance from the crack tip at which the probability of cleavage cracking exhibits a maximum. This is illustrated in Figure 9 where probability of failure is plotted as a function of distance. The probability approaches zero at the crack tip because the volume of material containing cracked carbides tends to zero. At distances greater than $X_{c}$ the probability decreases with distance because $\sigma_{y y}$ is decreasing. Evans and Hutchinson derived the following expression for $X_{c}$.

$$
x_{c}=\frac{2}{9}\left[\frac{5(N+1) k}{2(m-k(N+1))}\right]^{N+1}\left(\frac{\sigma_{C}}{\sigma_{f}}\right)^{N+1}\left(\frac{K_{I C}}{\sigma_{0}}\right)^{2}
$$

where $N$ is the hardening exponent

( $N=1 / n$, where $n$ is the work hardening exponent)

$k$ is a constant $\leq 1$

$m$ is a shape parameter characteristic of the carbide size distribution.

Their model also resulted in an equation for $K_{I C}$ as a function of material properties:

$$
K_{I C}=A\left[\begin{array}{ll}
\sigma_{f}^{(N+1) / 2} & r_{C}^{3 / 2(k+1)} \\
\hline(f l)^{1 / 2(k+1)} & \sigma_{0}(N-1) / 2
\end{array}\right]
$$

where

$$
\begin{aligned}
& f \text { is the volume fraction of carbides at the grain boundary. } \\
& r_{C} \text { is the average carbide radius }
\end{aligned}
$$




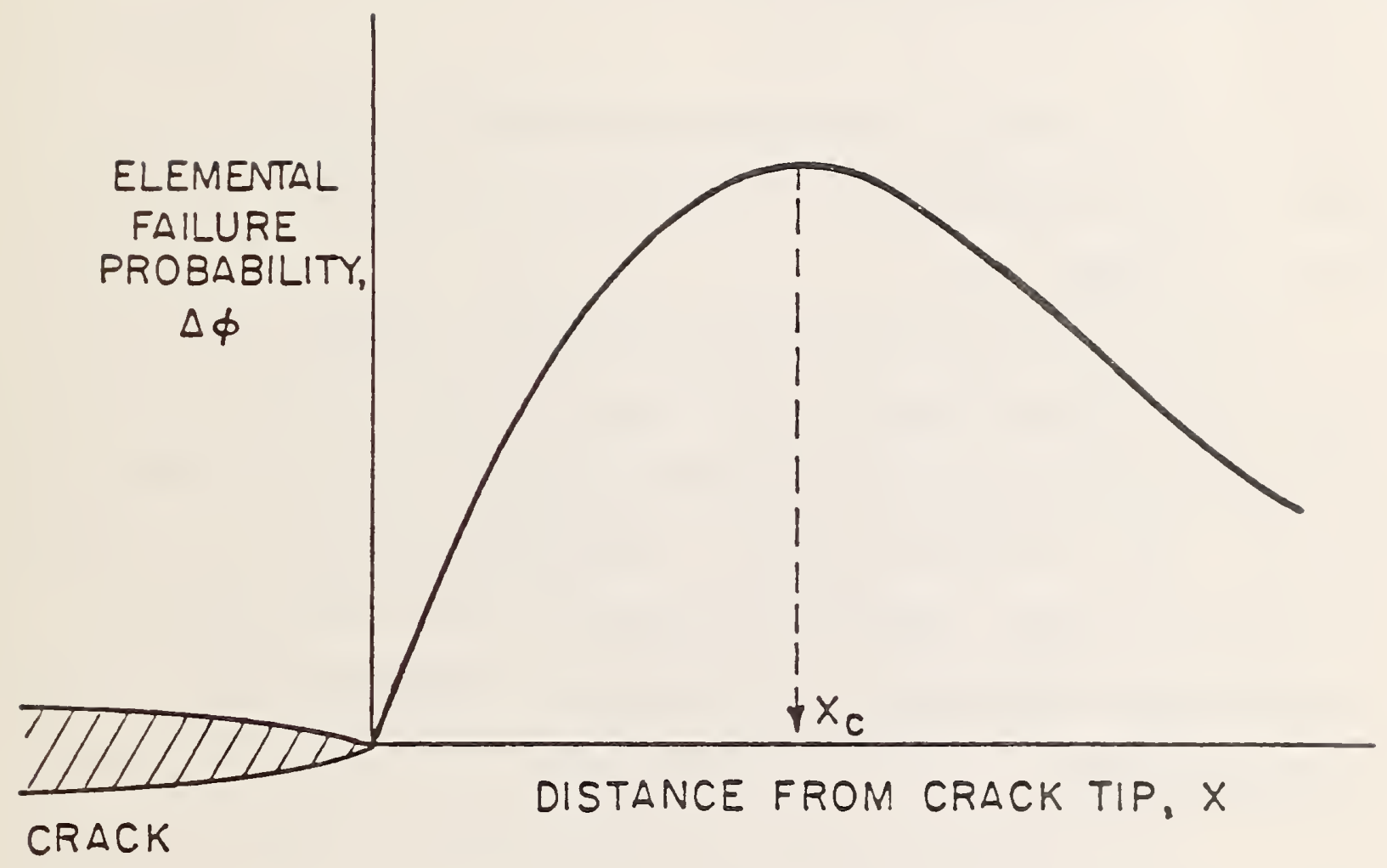

Figure 9. Probability of failure as a function of distance

[40]. The distance at which probability is maximized is defined as the critical distance. 
$\ell$ is a characteristic distance along the crack front

$A$ is a function of $N, k, m$, and $\ell$.

It appears from equation (25) that $X_{c}$ depends on yield strength and is therefore a function of temperature. However, when the yield strength dependence of $K_{I C}$ from equation (26) is substituted into equation (25) the $\sigma_{0}$ terms cancel; i.e.,

$$
x_{c} \propto \sigma_{0}^{0}
$$

This observation, that $x_{c}$ is not temperature dependent, is very important to the present study as will become evident in the Results and Discussion chapter.

Rosenfield, et. al. $[41,42]$ have experimentally measured $x_{c}$ by finding the fracture-triggering particle with a scanning electron microscope (SEM) and measuring the distance from the crack tip to the particle. These particles were either grain boundary carbides or nonmetalic inclusions. Although the measured $x_{c}$ values were quite scattered, the values appear to be independent of temperature and fracture toughness.

\subsubsection{Ductile Tearing (Microvoid Coalescence)}

In ferritic steels as the temperature increases and the flow stress decreases, it becomes more difficult to produce enough constraint in the plastic zone for the fracture stress to be exceeded. At temperatures where the conditions become unfavorable for cleavage a ductile fracture mechanism, microvoid coalescence, operates. This is the dominant 
fracture mechanism of FCC alloys, even at very low temperatures. The commonly observed microstructural stages on the initiation and growth of a fibrous crack are [43]:

a) The formation of a free surface at a second-phase particle or inclusion by either interface decohesion or particle cracking.

b) Growth of a void around the particle, often under hydrostatic stress.

c) Coalescence of the growing void with the crack tip. These events are thought to occur almost continuously as the crack advances; that is, second-phase particles progressively enter the "process zone" ahead of the crack tip, and progressive void growth and coalescence produce small increments of crack growth. The microstructural parameters that control toughness are the spacing, $x_{0}$, of the particles and the fracture strain of the matrix, $\varepsilon_{f}[24,44,45]$.

When voids grow in a hydrostatic tensile stress field an internal neck is formed between the void and the crack tip, and final separation occurs along a knife edge in three dimensions to give a fracture surface composed of cusps and dimples centered on second-phase particles.

\subsubsection{The Ductile-to-Brittle Transition}

The effect of flow stress on the ductile-to-brittle transition is illustrated schematically in Figure 10. Flow stress is highly temperature dependent while fracture stress is insensitive to temperature changes. Cleavage occurs when the flow stress exceeds the 

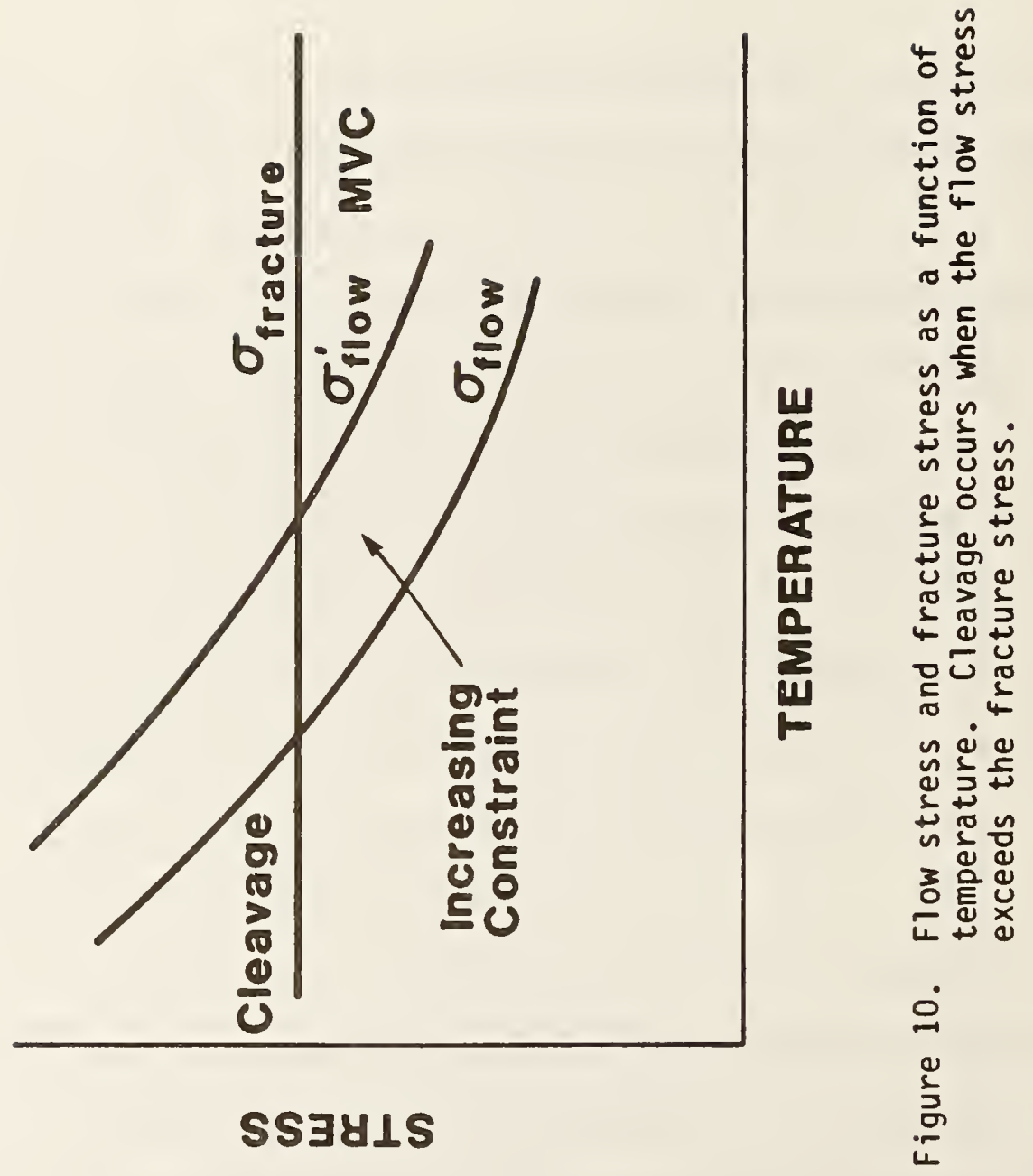
fracture stress. For the simple schematic in Figure 10 the ductile-tobrittle transition occurs where the flow stress curve crosses the fracture stress curve. An increase in triaxiality elevates the flow stress, causing the transition to occur at a higher temperature.

The effect of the flow properties of material near the crack tip on fracture toughness and micromechanism of fracture is further illustrated in Figure 11. At a low temperature, represented by curve(a) in Figure 11, the yield strength is high; only a small degree of plastic flow occurs before the critical fracture stress is reached. At higher temperatures (curve(c)) the yield strength is so low that the fracture stress is never reached, even after significant plastic flow. The crack begins to grow by ductile tearing when the material ahead of the crack tip reaches a critical strain. Curve(b) represents an intermediate condition. The material reaches the critical strain, causing stable crack growth. Upon further strain hardening the material reaches the fracture stress, resulting in unstable cleavage.

Curve(a) represents lower-shelf behavior because a small degree of plastic flow has preceded fracture; small-scale yielding prior to fracture results in a low toughness value. Curve(c) represents upper-shelf behavior because fracture is by ductile tearing and a high degree of plastic flow (corresponding to high toughness) precedes failure. 


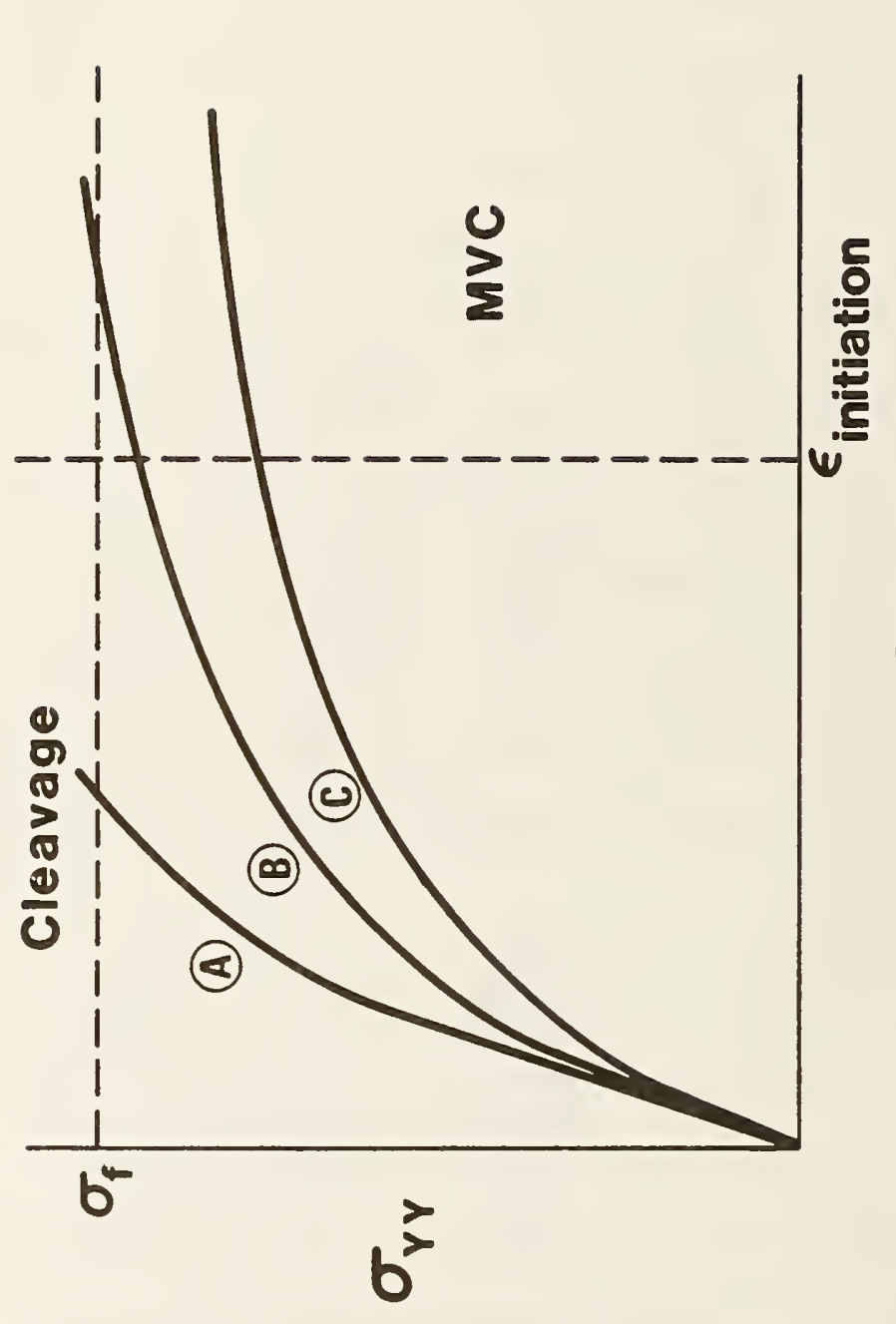

ธิ㇒士

4

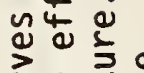

ว

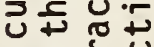

उ五

는

$4+00$

는

올

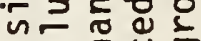
经过

응

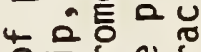

田

>

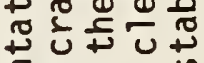

政

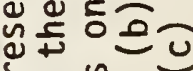

包

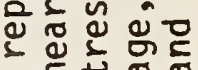
过苾 중

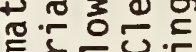
है क्षे

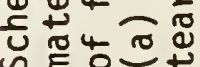
UEO

ت 
1.4 Effect of Specimen Size and Geometry on Fracture Behavior

Specimen geometry can have a substantial effect on fracture toughness as illustrated in Figure 12. As thickness, crack length, and uncracked ligament length become large compared to the plastic zone the fracture toughness reaches a minimum value defined by $K_{I C}$. The ASTM E 399 standard is designed to measure this lower bound fracture toughness.

The size effect is most pronounced in the ductile-to-brittle transition region, as $17 l$ ustrated in Figure 13 [46]. The transition tends to occur at higher temperatures with larger specimens. At a constant temperature in the transition region, represented by the vertical 1 ine in Figure 13 , the small specimen has a higher fracture toughness than the large specimen.

This represents a potentially dangerous situation when using fracture toughness data from small specimens to design large structures. On the upper shelf of toughness, where ductile tearing is the micromode of fracture, it is permissible to predict the fracture toughness of large structures from small specimen data because $J_{i}$ and $\delta_{i}$ are not strong functions of geometry $[47,48]$. However, in the transition region where cleavage may be the micromode of fracture, a crack in a small specimen may grow in a stable and ductile manner while a large structure could fail catastrophically by cleavage and have much lower toughness than predicted in the small scale test.

Although a $K_{I c}$ test will always give lower-bound results, it is sometimes not practical or possible to perform a fracture test according 

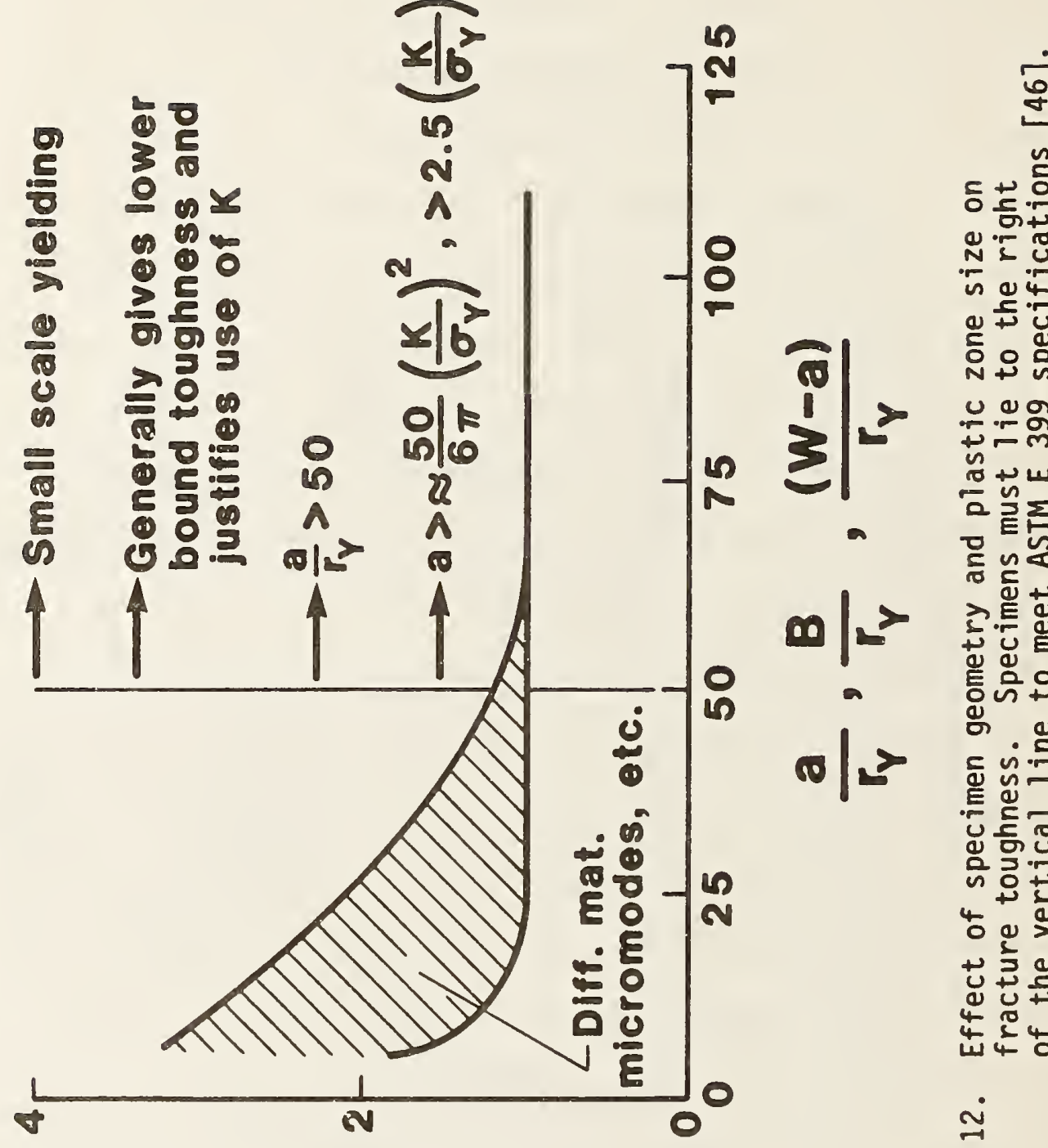

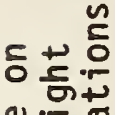

닝

N

in 0

동워

의

$+\infty \mathrm{m}$

吕山س

完资

음 年

叫

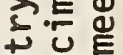

㽞

응ㅇ

बั

들 出

든

헝

的实昰

$40 \%$

$+3$

Q

48

出被

ำ

ํำ

$\left.\right|_{-5} ^{15}$ 


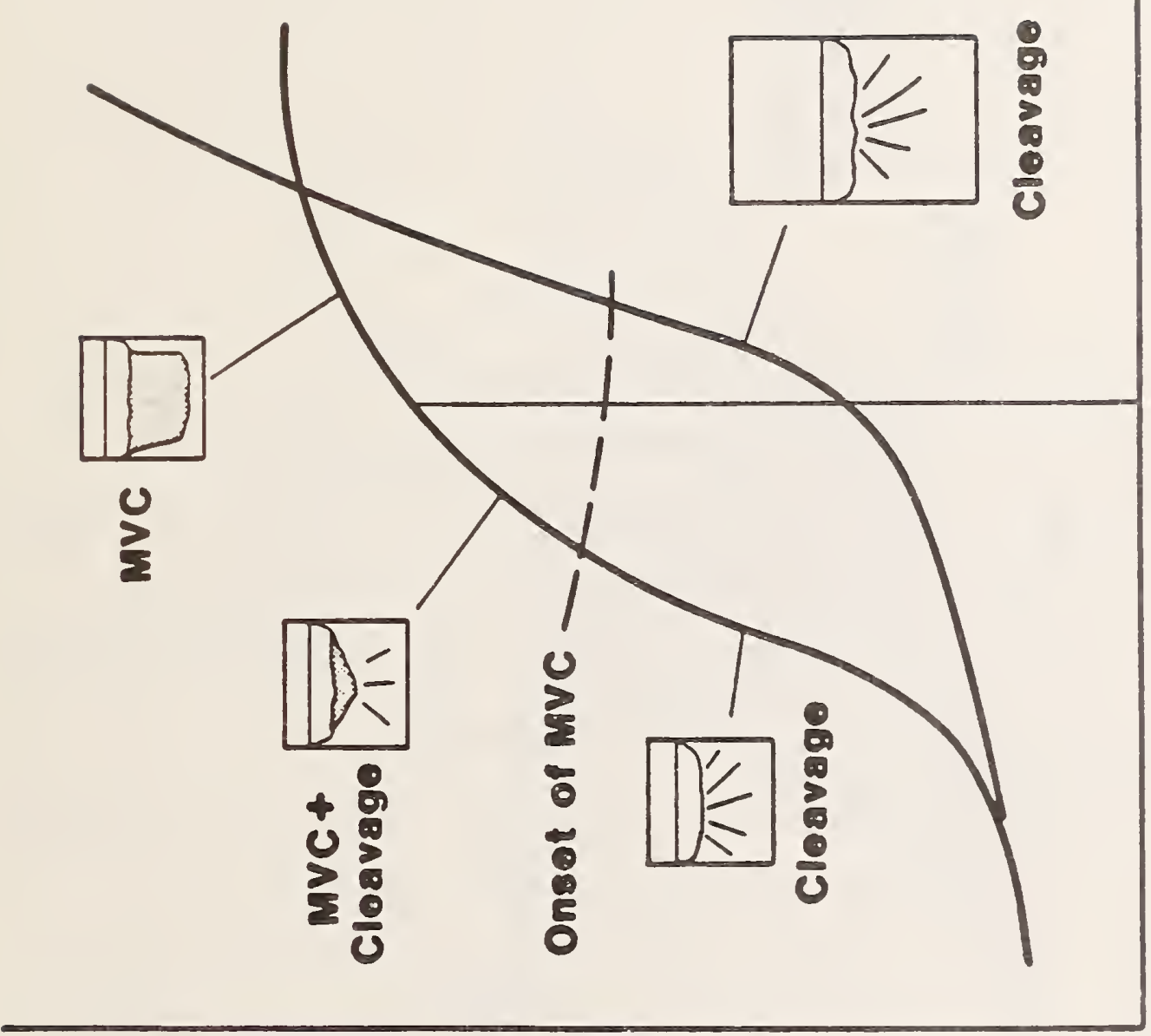

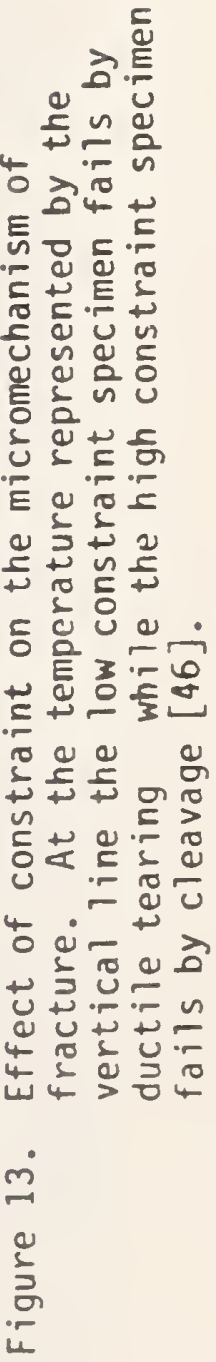

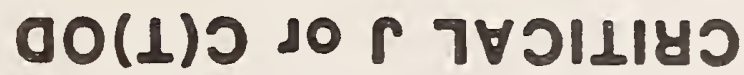


to $\mathrm{E} 399$ specifications. For low- and medium-strength steels the size requirements for a valid $K_{I c}$ test are often excessive; a specimen of suitable size would be very expensive and would probably exceed the load capacity of most test machines. Landes [49] has shown that for some materials a valid $K_{I c}$ cannot be obtained, even with an infinitely large specimen.

A structure which operates in the ductile-to-brittle transition region will often behave in an elastic-plastic manner. Therefore a $\mathrm{K}_{\mathrm{IC}}$ value, which is based on a linear elastic analysis, would be overly conservative.

\subsubsection{Experimental Observations of the Size Effect}

A number of investigators [47-55] have studied the effect of specimen thickness on the fracture toughness of various materials. In the transition region, toughness decreases with increasing thickness until a saturation thicknss (plane strain) is reached; further increases in thickness have little or no effect on toughness.

The relative crack length and ligament length in bend specimens can also affect fracture toughness in the transition region. Figure 14 schematically shows the consensus results of a number of investigators [47-53,56-59]. The ductile-to-brittle transition temperature tends to increase with increasing crack-length-to-width ratio, a/W. For very deep cracks, fracture toughness can increase due to plastic flow to the nearest free surface. 


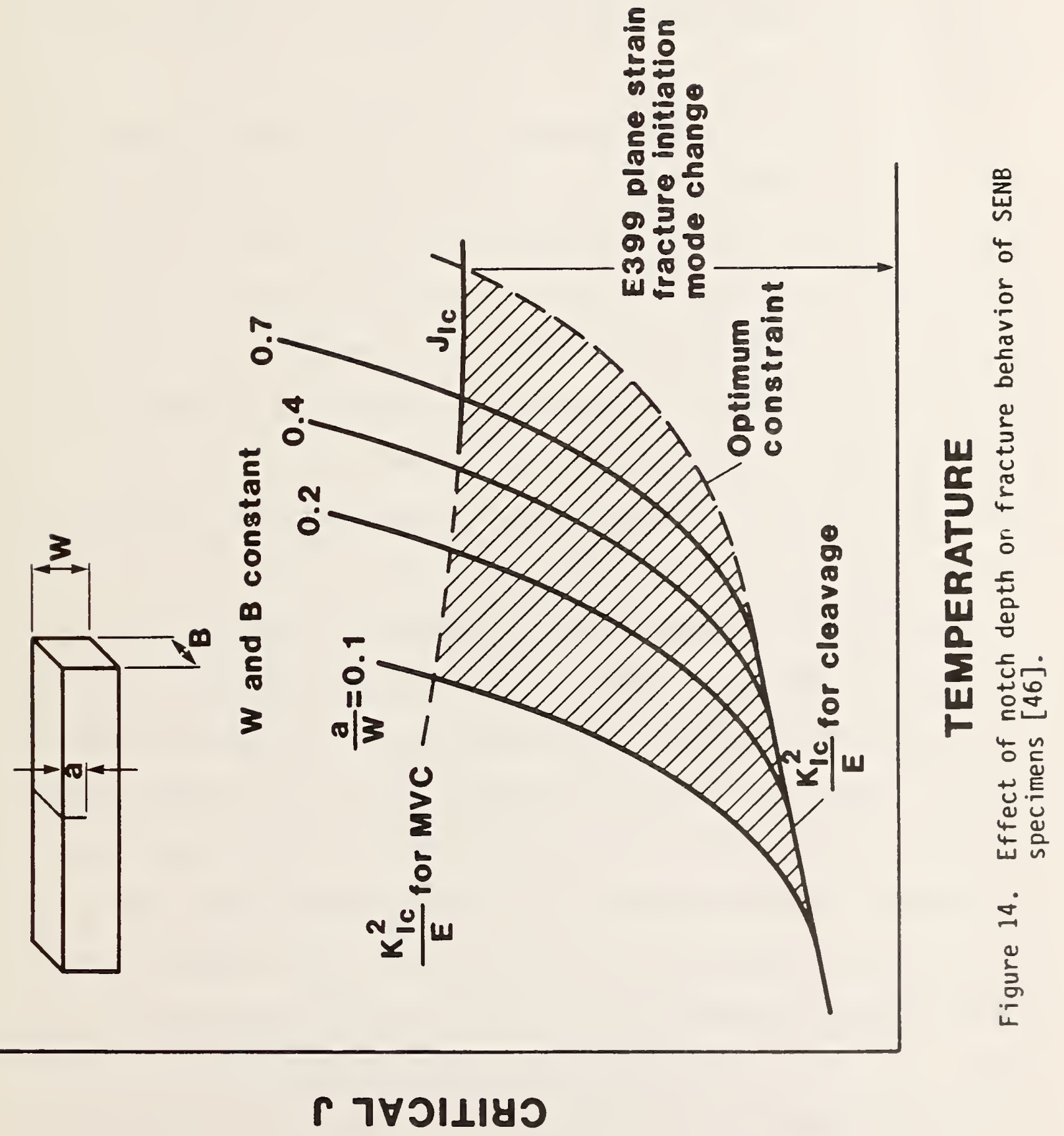




\subsubsection{The Causes of Size Effects}

There are two different explantions for the size effect on fracture toughness. One explanation is based on the statistical nature of cleavage fracture [60-63]; the second is related to the crack-tip stress state $[47-53]$.

Cleavage fracture toughness depends on the size and distribution of carbides or inclusions which serve as crack initiation sites $[24,42]$. A large particle near the tip of the major crack produces a large crack nucleus which results in a low fracture toughness. According to the statistical model $[60,61]$, the average fracture toughness of a large specimen is lower than that of a smaller specimen because the greater volume of material sampled in the large specimen results in a higher probability of finding a large, favorably oriented particle near the crack tip.

An alternative explanation for size effects is that of crack-tip constraint [47-53]. Due to the stress concentration inherent in a cracked body, the material near the crack tip, which is highly stressed, tends to contract perpendicular to the major stress axis. However, this material is constrained by the surrounding material due to compatibility requirments. This constraint produces a triaxial stress state near the crack tip which elevates the flow stress locally. This elevation in flow stress makes it easier for the material to reach the fracture stress. In a small fracture specimen a small amount of material surrounds the crack tip; therefore constraint is relatively low. In a 
large specimen or structure, constraint is high because a large volume of material surrounds the crack tip; thus, fracture toughness is lower because less elevation of flow stress by plastic deformation (i.e., strain hardening) is needed to reach the fracture stress. Elevation of the flow stress due to constraint shifts the ductile-to-brittle transition to higher temperatures (see Fig. 10).

Most authors who have studied size effects concede that both statistical and constraint effects can be contributing factors. However, there is some disagreement as to which of these two factors is dominant. It is the author's opinion that the dominant factor depends largely on the material. This point is further discussed in Section 3.5.

\subsubsection{Scaling Facture Toughness}

Several investigators have tried various methods to predict lower-bound toughness values from small-scale tests. One of the most common methods involves empirically correlating Charpy-impact energies with $K_{I c}$ values [64-67]. However, these empirical equations are generally only valid for the materials used for the correlations. None of these methods has proven to be accurate for a wide range of materials [68]. In a recent article, Dawes, et. al. [69」 compared critical crack lengths obtained by these methods with actual wide plate test results. They found that these empirical correlations can sometimes overestimate critical crack lengths by a factor of 10 or more. 
Landes and Shaffer [60] used the statistical concept to predict KIC values from small specimen results. They applied a Weibull [70] distribution to various populations of small specimen results to obtain lower-bound toughness values.

Rosenfield, et al. [41] have recently had success in predicting large-scale results from small-scale tests. They found that the critical $J$ for a large pressure vessel, $J_{C}$, was equivalent to the elastic portion of the critical J, $G_{C}$ for small specimens. There is, however, no theoretical basis for the contention that $G_{C}$ is a material constant. Also, this hypothesis has not been tested for a range of materials. It may only be a coincidence that the $G_{C}$ values for the specimen and the structure agree for a particular material.

All scaling methods known to the author are either purely empirical or are based solely on statistical considerations. No one has used the concept of constraint to quantitatively analyze size effects. Several investigators [71-76] have used two- and three-dimensional elastic-plastic finite element analyses to observe the crack-tip stress state. However, none of thse analyses were applied to the scaling problem.

\subsection{Statement of the Problem}

As stated above, fracture toughness in the ductile-to-brittle transition region is highly dependent on the size and geometry of the structure or test specimen. At a given temperature in the transition 
region, a small laboratory specimen may be ductile while a large structure of the same material at the same temperature may be quite brittle. In order to obtain a fracture toughness value representive of the structure at the service temperature, a laboratory specimen must be tested at some temperature below the service temperature. Typically, this temperature shift can range from $5^{\circ}$ to $50^{\circ} \mathrm{C}$, depending on the material, structure, and specimen. A method to quantify temperature shifts in the ductile-to-brittle transition must be developed if the safety of structures that operate in the transition region is to be assured.

\subsection{Objectives}

The major objective of this work was to develop a method to predict fracture toughness in the ductile-to-brittle transition as a function of specimen (or structure) size and geometry. This method is based on the concept of constraint at a crack tip which produces varying degrees of stress triaxiality.

\subsection{Technical Approach}

A physically-based model for fracture in the ductile-to-brittle transition region was developed to account for temperature shifts in the transition with specimen size. It was assumed that upward temperature shifts were caused by increases in crack-tip stress triaxiality. 
Most previous attempts to predict temperature shifts or lower-bound toughness values have been purely empirical. None of these methods are valid for a wide range of materials. The statistical model of Landes and Shaffer [60] is more rigorous but it ignores constraint effects. The present analysis is based on constraint concepts but does not consider statistical effects. An integrated model which considers both constraint and statistical effects will be left for future work.

The specific approaches used in this investigation are outlined below.

1) Experimental data. A relatively large body of experimental data was obtained in this study. Most of the data consist of fracture toughness values for various specimen configurations. These data provided an empirical demonstration of size and geometry effects on fracture toughness. The data were also useful for assessing the validity of models developed in this investigation. Fracture toughness data were obtained for two materials: a C-Mn steel and commercially pure iron.

2) Constraint measurements. Previous authors have discussed constraint in purely qualitative terms [47-53]. In the present study constraint was quantified. A technique was developed to experimentally measure crack-tip triaxiality. Fracture toughness data, microhardness tests, and existing models for cleavage fracture were utilized for this measurement. 
3) Spring model for constraint. The triaxial stress state near a crack tip was modeled by means of springs which act on a volume of material ahead of a crack.

4. Equation for the ductile-to-brittle transition. The model yielded an equation for fracture toughness (in the transition region) as a function of material properties and geometry-dependent parameters. This expression was used to construct ductile-to-brittle transition curves which were compared with experimental data.

5. Application to structures. Transition curves for a hypothetical structure were predicted by assuming worst-case values for the geometry-dependent parameters in the fracture toughness equation.

6. Application to other materials. The expression for fracture toughness in the transition region was applied to both materials, i.e., the C-Mn steel and the pure iron. 


\section{EXPERIMENTAL PROCEDURE}

\subsection{Test Materials}

The material used for the majority of the mechanical tests was a $25.4 \mathrm{~mm}(1.0 \mathrm{in})$ thick plate of ABS grade EH36 steel, a $350 \mathrm{MPa}$ (51 ksi) yield strength $\mathrm{C}-\mathrm{Mn}$ steel. The chemical composition is given in Table 1. The steel was in the normalized condition and had particularly uniform properties due to sulfide shape control. In addition to the normalized steel a hot-rolled billet of commercially pure iron was used for a limited number of tests. The chemical composition of this material is given in Table 2.

\subsection{Tensile Tests}

The tensile properties of both materials were determined from round tensile specimens $(6.35 \mathrm{~mm}$ diameter and $31.75 \mathrm{~mm}$ gage length) with the tensile axis parallel to the rolling direction. Those specimens were tested at various temperatures ranging from $-255^{\circ} \mathrm{C}$ to $25^{\circ} \mathrm{C}$. All tensile tests were performed in a screw-driven tensile test machine at a cross-head speed of $0.2 \mathrm{~cm} / \mathrm{min}$. The upper yield point, lower yield stress, ultimate tensile strength, percent reduction in area, percent elongation and true stress at fracture were measured for each test. All testing and measurement procedures conformed to the guidelines of ASTM E 8, the standard for tension testing of metals.

The testing machine was equipped with a fixture designed for cryogenic testing. Various temperatures were attained by an elaborate 


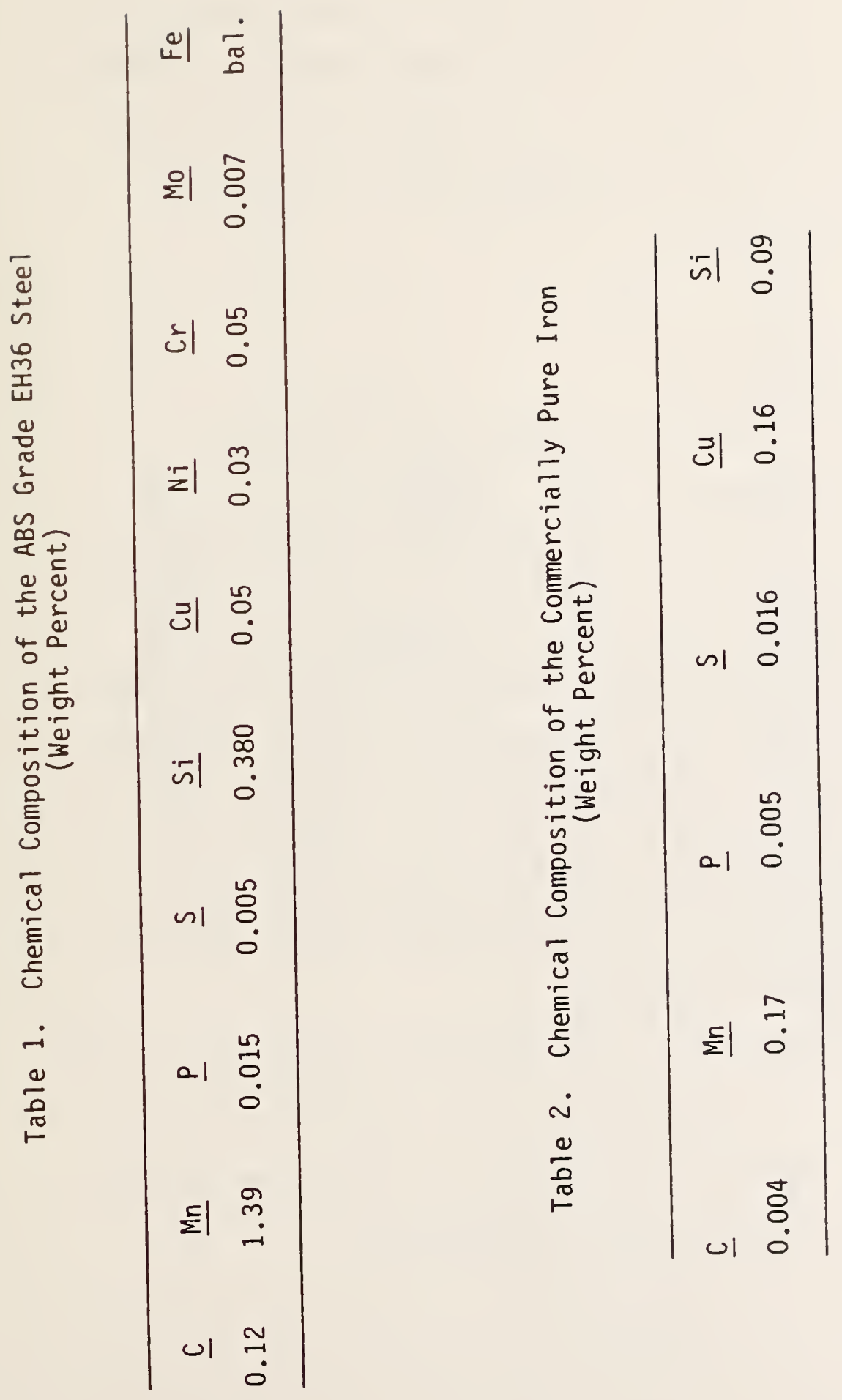


temperature control system. The specimen was cooled by reservoirs of liquid nitrogen or liquid helium above and below the specimen. The specimen grips were equipped with electric heaters which were connected to controllers. The desired temperature was simply dialed into the controllers. The combination of heating and cooling the specimen produced a steady-state temperature.

\subsection{Charpy V-Notch Impact Tests}

The Charpy-impact transition curve was established for the normalized steel in the temperature range -196 to $25^{\circ} \mathrm{C}$. The notch orientation with respect to the rolling direction is shown in Figure 15. The procedures of ASTM E 23, the standard for impact testing, were followed. Deviations from the standard occurred in some tests. These deviations are noted in the results and discussion chapter. For each test the absorbed energy, percent shear and lateral expansion were measured. The latter two quantities were measured according to the guidel ines which appear in ASTM A 370. The maximum lateral expansion was measured with a digital micrometer.

The various test temperatures were attained by placing the specimens in a constant temperature bath (1iquid nitrogen or a liquid nitrogen methanol mixture) for a minimum of ten minutes. Each specimen was then removed form the bath and tested with in five seconds. 


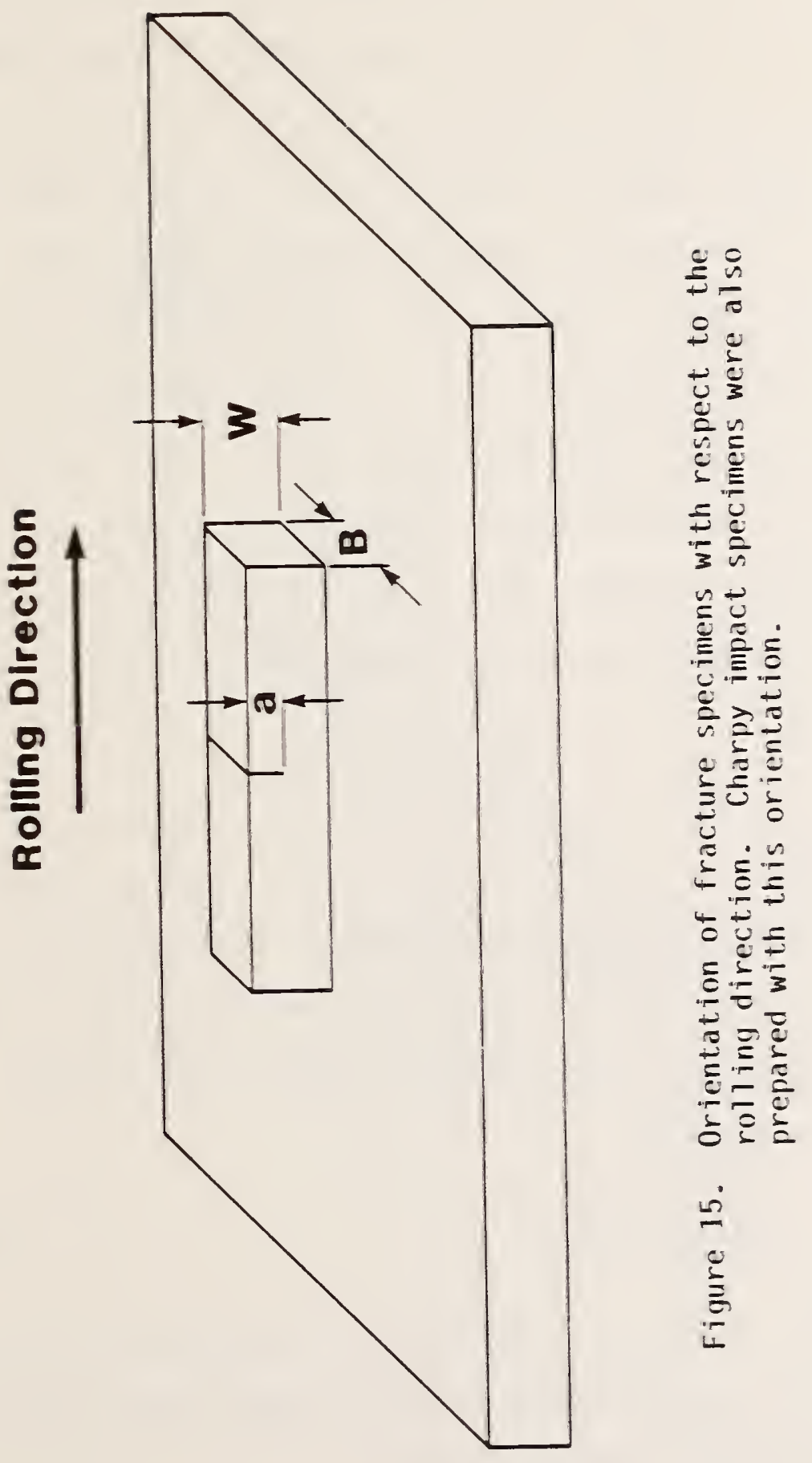




\subsection{Fracture Toughness Tests}

The ductile-to-brittle transition curves were established as a function of constraint for ABS EH36 steel and commercially pure iron. Constraint was varied by varying the specimen thickness ( $B)$, the crack length (a), the specimen width ( $W$ ), and the type of loading (tension vs. bending). A total of ten fracture specimen configurations of the normalized steel were tested. This includes seven geometries of singleedge notched bend (SENB) specimens (see Fig. 2) and three geometries of single-edge notched tension (SENT) specimens (see Fig. 16). Fracture toughness tests were also performed on two geometries of pure iron SENB specimens. The fracture toughness test matrix for both materials is shown in Table 3. All specimens were machined with the notch orientation shown in Figure 15.

\subsubsection{SENB Specimens}

A11 SENB specimens were fatigue precracked at room temperature according to the specifications in the British standard for COD testing [19]. The maximum allowable stress intensity for fatigue loading, $K_{f}(\max )$, is given by:

$$
K_{f}(\max )=0.63 \sigma \sigma B^{\frac{1}{2}}
$$

where $\sigma_{y}$ is the yield strength (in $\mathrm{MPa}$ ) and $B$ is the specimen thickness (in $\mathrm{mm}$ ). The British standard states that the minimum stress intensity, $K_{f}(\min )$, be at least $10 \%$ of $K_{f}(\max )$, so that: 


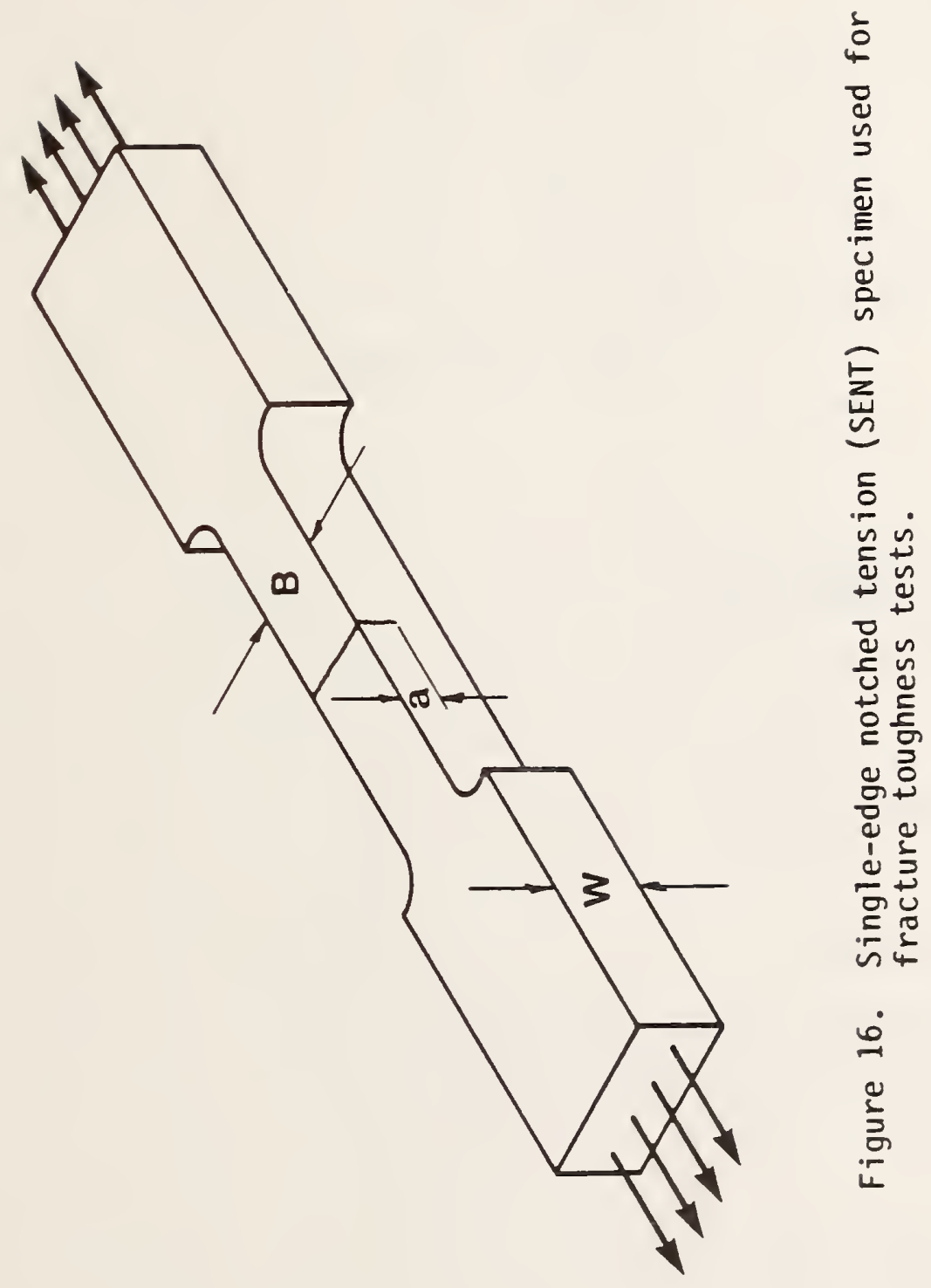


Table 3. Test Matrix for Fracture Toughness Tests.

I ABS EH36 Steel

\begin{tabular}{|c|c|c|c|c|}
\hline$a / W^{B}$ & $12.7 \mathrm{~mm}$ & $25.4 \mathrm{~mm}$ & $38.1 \mathrm{~mm}$ & $76.2 \mathrm{~mm}$ \\
\hline 0.2 & $b$ & $b, t$ & $b$ & \\
\hline 0.5 & & $b, t$ & & $b, t$ \\
\hline 0.75 & \multicolumn{5}{c|}{$(W=25.4 \mathrm{~mm})$} \\
\hline
\end{tabular}

\begin{tabular}{|c|c|}
\hline$a / W$ & $25.4 \mathrm{~mm}$ \\
\hline 0.2 & $b$ \\
\hline$(W=15.9 \mathrm{~mm})$
\end{tabular}

\section{Pure Iron}

\begin{tabular}{|c|c|c|}
\hline$a / W$ & $25.4 \mathrm{~mm}$ & $50.8 \mathrm{~mm}$ \\
\hline 0.2 & $b$ & $b$ \\
\hline \multicolumn{3}{|c|}{$(W=25.4 \mathrm{~mm})$}
\end{tabular}

$$
\begin{aligned}
& b \text { - Bend (SENB) specimen } \\
& t \text { - Tension (SENT) specimen }
\end{aligned}
$$




$$
\Delta K_{f} \leq 0.9 K_{f}(\max ) .
$$

All tests were performed in displacement control on a $100 \mathrm{kN}$ servohydraulic test machine. The displacement rate in all tests was $0.80 \mathrm{~mm} / \mathrm{min}$. This corresponds to a loading rate (in the elastic range) for the square section $(W=B$ ) specimens of approximately $9.3 \mathrm{kN} / \mathrm{s}$ for shallow notched $(a / W=0.25)$ specimens and $375 \mathrm{~N} / \mathrm{s}$ for the deep notched $(a / W=0.75)$ specimens. The experimental setup is shown in Figure 17. The mouth-opening displacement and the load-line displacement were measured simultaneously during the test by two clip gages. The load-line displacement was measured by the comparison bar technique developed by Dawes $[7 i]$. The load and the two displacements were recorded on a two-pen $X-Y$ plotter. The test instrumentation was wired to a minicomputer through an analog-to-digital converter. The load, crosshead displacement, and both clip-gage displacements were recorded by the computer at approximately 0.3-s intervals and stored on a magnetic disk. The computer typically collects and stores around 500 sets of data in a 3 to 5 minute bend test.

Low temperatures were attained by attaching a box to the lower crosshead and filling it with either liquid nitrogen or an alcohol and dry ice mixture. Intermediate temperatures (between -196 and $-70^{\circ} \mathrm{C}$ ) were attained by pouring liquid nitrogen into the box to a level below the specimen. The specimen was cooled by heat transfer through the test fixture and by the vapor coming off of the boiling nitrogen below the specimeri. The actual specimen temperature was measured by a thermocouple implanted in the specimen. 


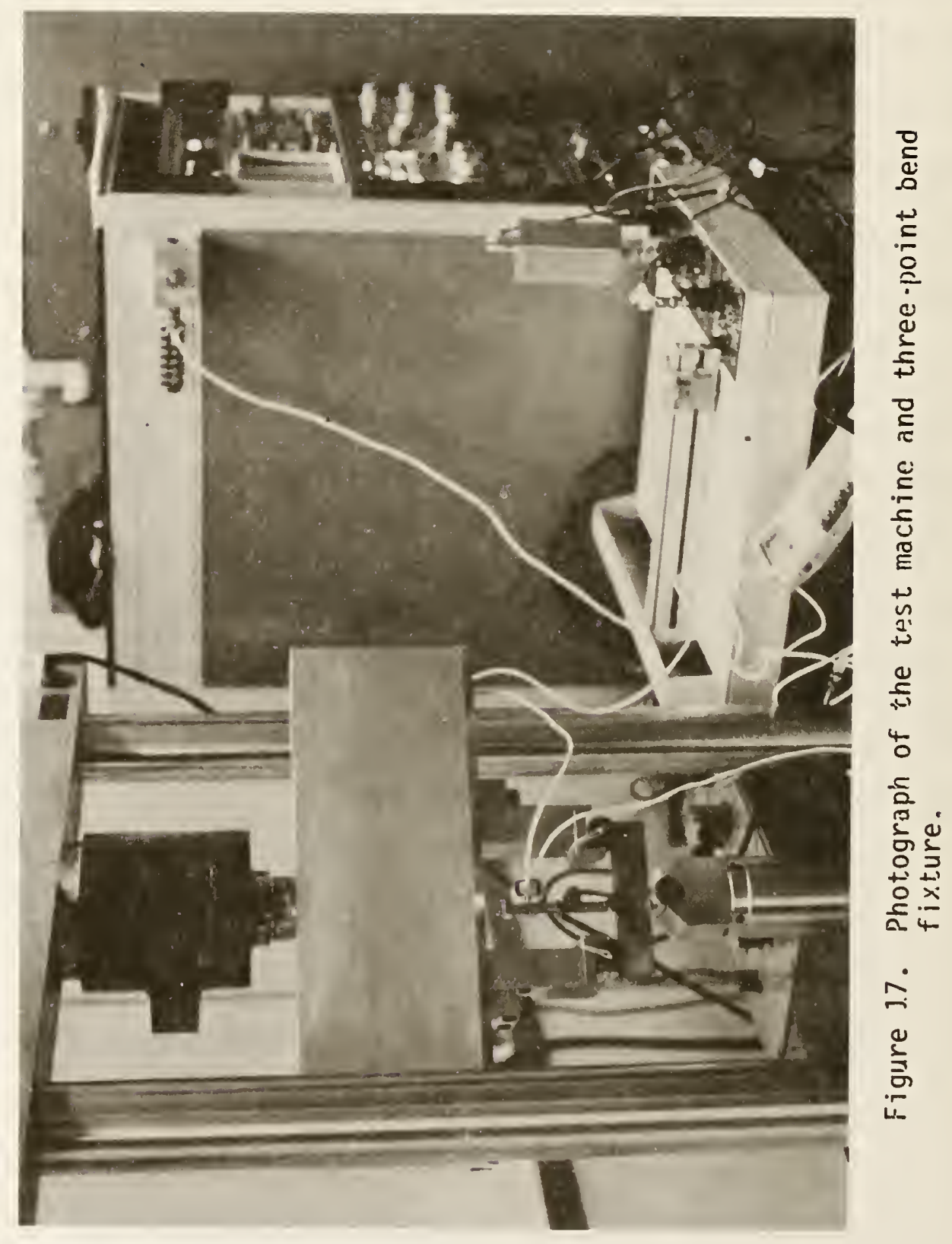


In each test, fracture toughness was measured by the two EPFM parameters, $J$ and CTOD. Figure 3 shows the notation used for critical values of CTOD. The notation depends on the nature of the fracture event, i.e. whether the crack extension is brittle or ductile, and whether or not unstable cleavage is preceded by stable crack growth. A similar notation was used for reported critical values of the J-integral (see Fig. 4).

The crack-tip opening displacement (CTOD) was computed from the following relationship:

$$
\delta=\delta_{e}+\delta_{p} \quad=\frac{k^{2}}{2 \sigma_{y} E}+\frac{r_{p}(W-a) V_{p}}{r_{p}(W-a)+a+z}
$$

where $v_{p}=$ the plastic component of the mouth-opening displacement

$$
\begin{aligned}
& z=\text { knife edge thickness } \\
& r_{p}=\text { rotational factor. }
\end{aligned}
$$

This equation separates CTOD into elastic and plastic components ( $\delta_{e}$ and $\delta_{p}$, respectively). The British standard [18] suggests that a value of 0.4 be assumed for $r_{p}$ in equation (30). However, a more precise value for the rotational factor can be calculated if the plastic components of load-line displacement and mouth opening displacement $\left(q_{p}\right.$ and $V_{p}$, respectively) are known [78]:

$$
r_{p}=\frac{1}{W-a}\left\{\frac{V_{p} W}{q_{p}}\left(1-\frac{q_{p}}{16 W}\right)-(a+z)\right\}
$$


The average value of $r_{p}$ was found to be 0.5 for the normalized steel and 0.38 for the pure iron. These values were used in equation (30) for al 1 CTOD calculations.

The ASTM Standard E 813, the standard for $J_{I C}$ testing, recommends that the following equation be used to estimate the J-integral for an SENB specimen.

$$
J=\frac{2 U}{B(W-a)}
$$

where $U$ is the area under the load/load-1 ine displacement curve. However, $J$ can also be estimated from an equation derived by Sumpter and Turner [79]:

$$
J=\frac{K^{2}}{E^{\prime}}+\frac{2 U_{p}^{v}}{B(W-a)}\left[\frac{W}{r_{p}(W-a)+a+z}\right]
$$

where $U_{p}^{v}$ is the area under the load/mouth-opening displacement curve. Both equation (32) and equation (33) were used to compute values of $J$. The results are compared in the Appendix.

The method that is utilized to detect the onset of stable crack growth is the double displacement method. Both the mouth-opening displacements, $V$, and the load-line displacements, $q$, from each test are stored on a magnetic disk. A program has been written which computes $d q / d V$ and $d^{2} q / d V^{2}$, and plots them as a function of $V$. The critical displacement is inferred from these plots (see Appendix). 


\subsubsection{SENT Specimens}

The SENT specimens (Figure 16) were fatigue-precracked in bending with a span equal to $8 \mathrm{~W}$. The fatigue loads were chosen such that tension specimens had the same minimum and maximum $K_{f}$ values as the SENB specimens.

The SENT specimens were tested in a $1000 \mathrm{kN}$ servohydraulic test machine. All tests were performed in displacement control at a displacement rate of $1.60 \mathrm{~mm} / \mathrm{min}$. Each end was gripped by a pair of wedges which were held in place by a cylindrical steel collar. The wedge grip assembly is shown schematically in Figure 18. The specimen was cooled by spraying liquid nitrogen onto the specimen. The specimen was insulated by a styrofoam box placed around the specimen. The temperature was monitored by two thermocouples placed on opposite sides of the specimen, near the crack tip.

Critical values of crack-tip opening displacement and the J-integral were measured in the ductile-to-brittle transition region. Critical CTOD values were measured by means of two clip gages, one at the crack mouth and one $6.43 \mathrm{~mm}$ above the crack mouth. Figure 19 shows a schematic of the double clip-gage measurement of CTOD as well as the equations which were used. This method was verified by replication (see Appendix). 


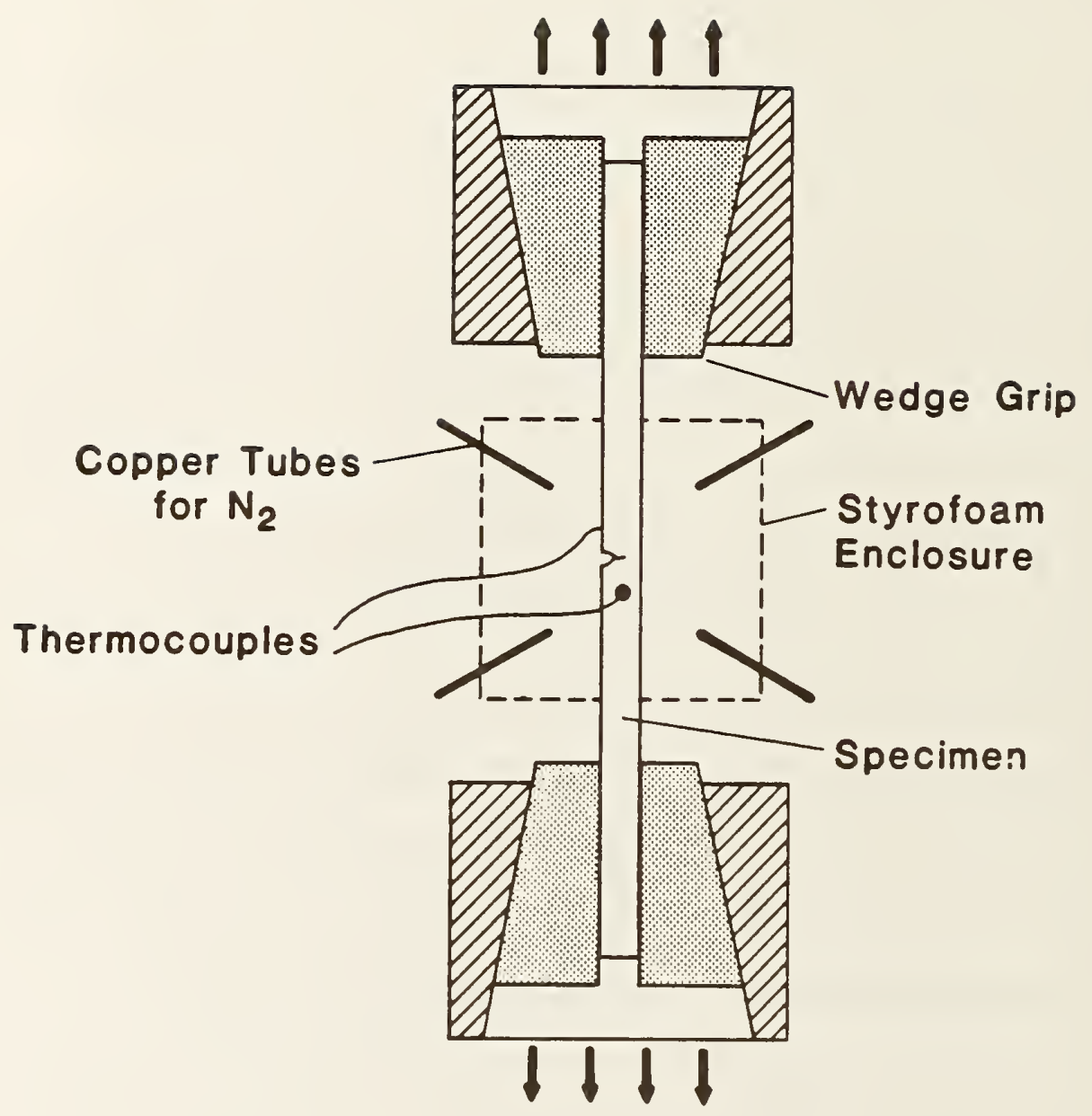

Figure 18. Schematic of the experimental setup for SENT tests. 


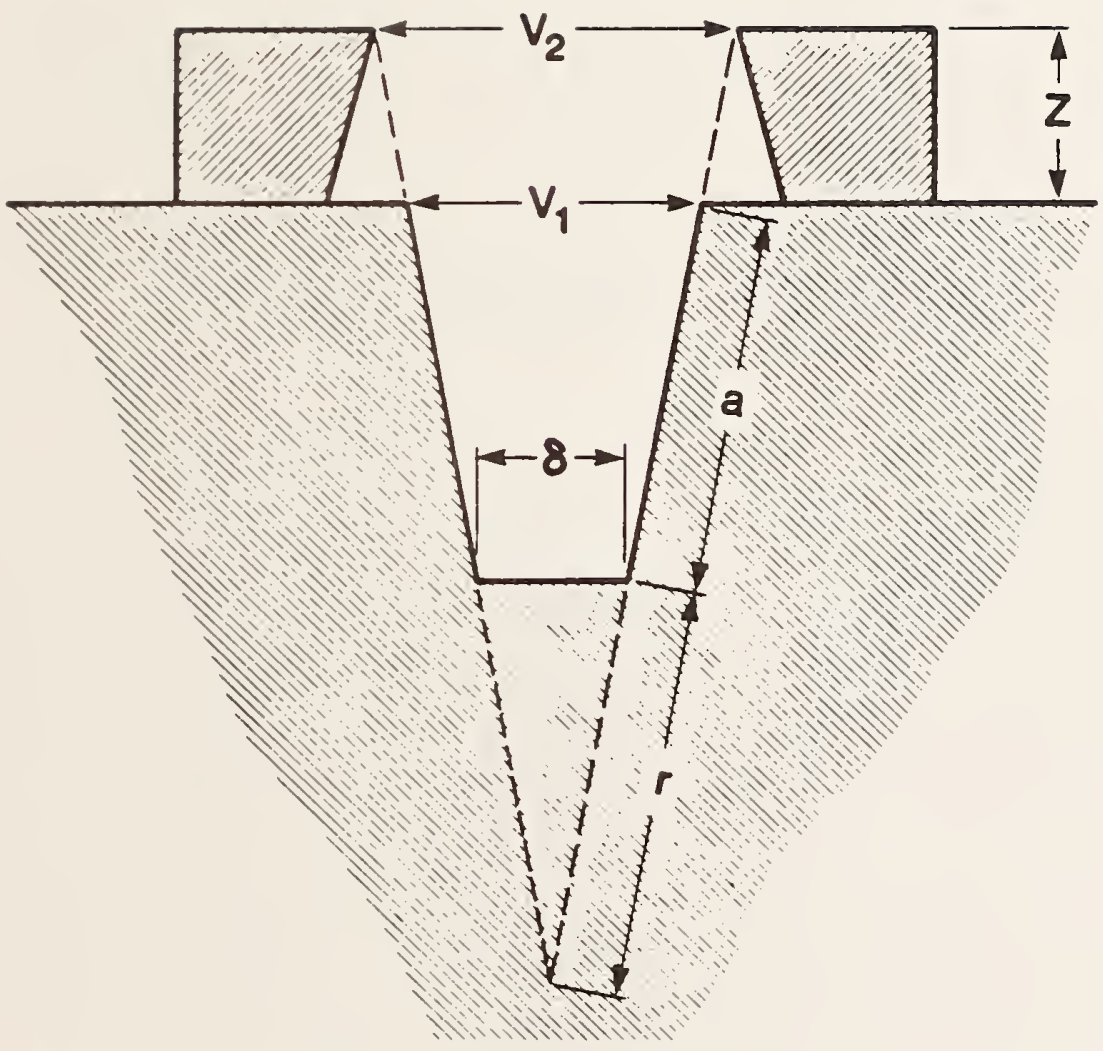

$$
\begin{aligned}
& 8=\frac{V_{1} r}{r+a} \\
& r=\frac{z}{\frac{V_{2}}{V_{1}}-1}-a
\end{aligned}
$$

Figure 19. The double clip gage measurement of CTOD from SENT specimens. 
The J-integral was estimated form an equation derived by Rice, et. a). [80]:

$$
J=\frac{K^{2}}{E^{\top}}+\frac{1}{B(W-a)}\left[2 \int_{0}^{V} V_{p d V}-P V_{p}\right]
$$

where $V_{p}$ is the plastic component of the clip-gage displacement. Equation (34) was derived for a cracked plate loaded in tension. It is assumed in this equation that the specimen is restricted from bending. This condition was imposed by the wedge grips (Fig. 18). 


\section{RESULTS AND DISCUSSION}

\subsection{Tensile Data}

The tensile properties of the ABS grade EH36 steel for 10 temperature ranging from $-196^{\circ} \mathrm{C}$ to $25^{\circ} \mathrm{C}$ are shown in Figure 20 and Table 4 . The data follow the expected trends with a minimal amount of scatter. Both the yield strength and the ultimate tensile strength increase markedly with decreasing temperature. All flow curves exhibited an upper and lower yield point which is characteristic of low-carbon steels. The yield strength (as plotted in Fig. 20) was defined as the upper yield point. This definition of yield strength was used to satisfy requirements of ASTM E 8. However, the lower yield stress is more indicative of the yielding behavior of a fracture specimen. The increment of stress between the lower and the upper yield stress is needed in a uniaxial tension specimen to generate mobile dislocations. The crack tip of a fracture specimen serves as a dislocation source; thus, the additional increment of stress is not needed for yielding to occur. The yield stress, $\sigma_{0}$, which appears in a number of equations and figures in this document refers to the lower yield stress.

The tensile properties of the commercially pure iron are shown in Figure 21 and Table 5. When this material was tested at temperatures above $-60^{\circ} \mathrm{C}$ the resulting flow curves are smooth; i.e., there is not a yield point. However, at temperatures below $-60^{\circ} \mathrm{C}$, a knee occurs at yield in the pure iron flow curves. This knee becomes sharper and more 
Is 기 'SS34 15

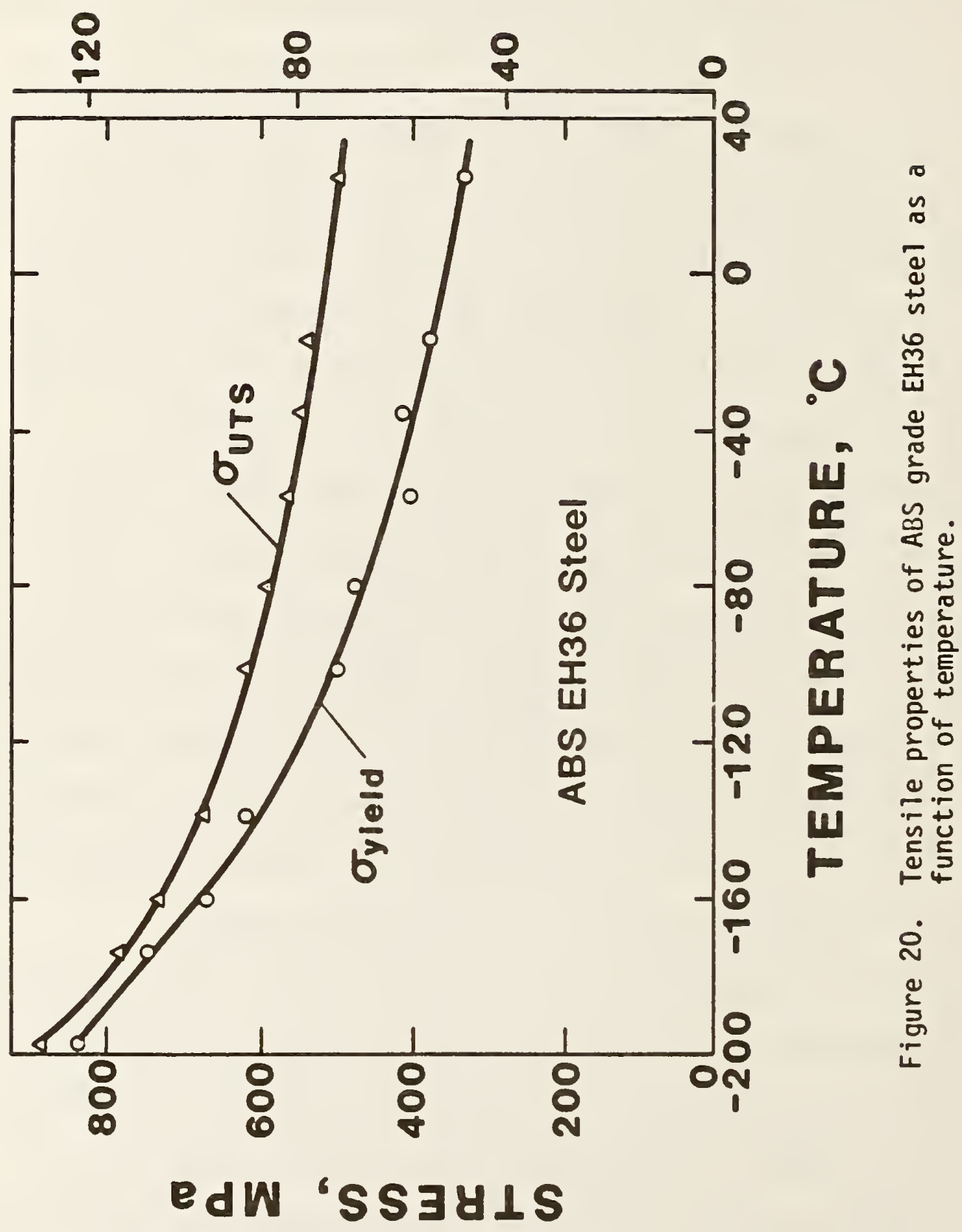




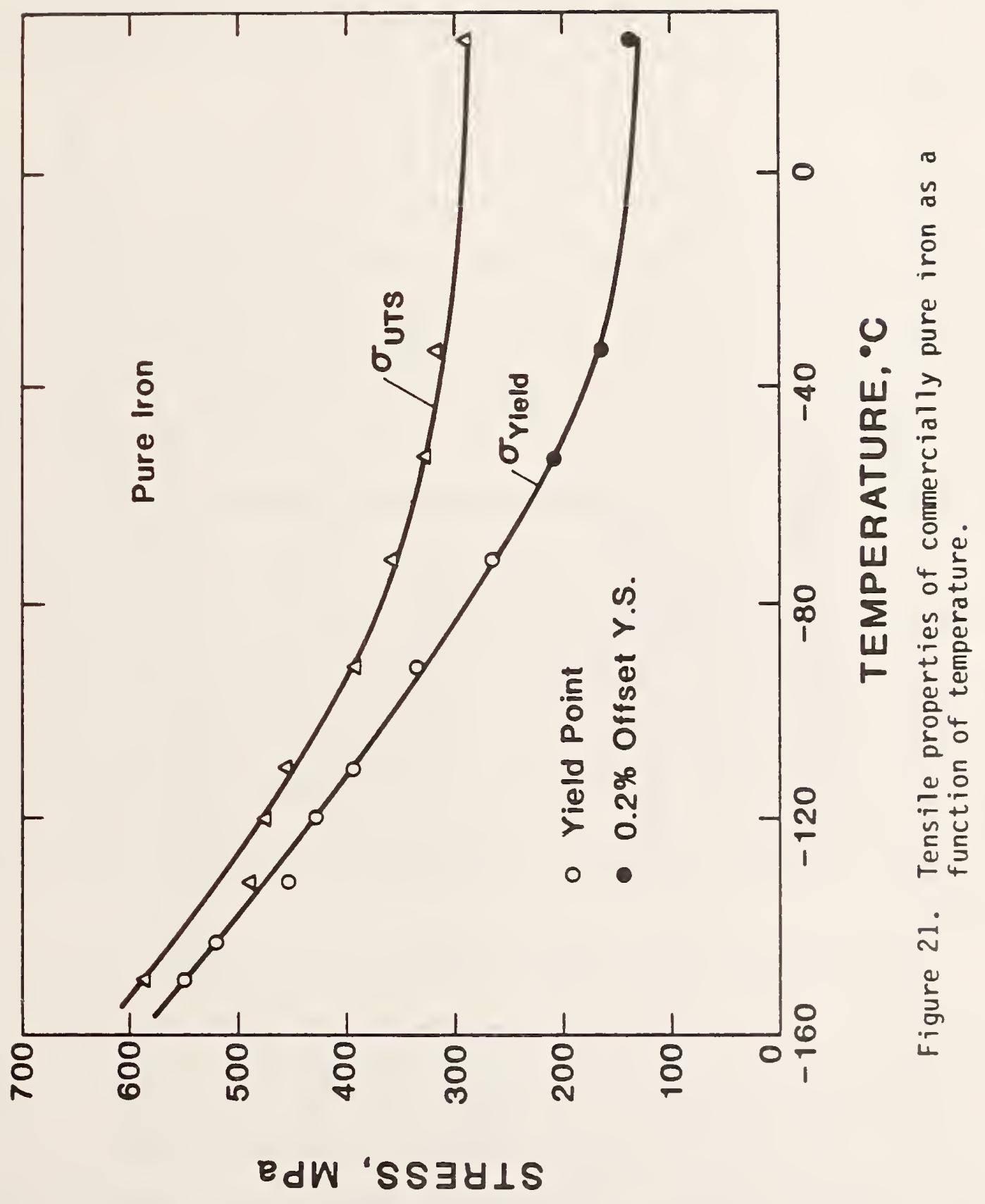


defined as the temperature is decreased. There is only a slight load drop after initial yielding; i.e. upper yield stress $\cong 1$ ower yield stress. The yield strength (as reported in Fig. 21 and Table 5) was defined as the 0.2 percent offset stress above $-60^{\circ} \mathrm{C}$ and as the yield point below $-60^{\circ} \mathrm{C}$.

\subsection{Charpy-Impact Data}

The Charpy $V$-notch impact energy transition curve was established for the ABS EH36 steel and is shown in Figure 22. The percent shear and lateral expansion were also measured for each specimen. The Charpy data are summarized in Table 6 . Figure 22 shows that the ductile-to-brittle transition occurs at about $-70^{\circ} \mathrm{C}$. Note that the curve is nearly vertical at this temperature. Most specimens tested at or near this temperature exhibited either fully-brittle (10w energy) or fully-ductile (high energy) behavior. Only a few specimens exhibited intermediate behavior. Table 6 shows that the transition from low energy to high absorbed energy corresponds well with the fracture surface transition ( $0 \%$ shear to $100 \%$ shear) and the transition from small to large 1 ateral expansion.

Because of the high toughness of this material, most of the upper shelf energy values are invalid according to the ASTM E 23 Standard. The full-scale energy of the Charpy test machine used in this experiment is $358 \mathrm{~J}$. According to the ASTM standard the energy absorbed by an impact specimen should not exceed $80 \%$ of the full-scale energy of the 


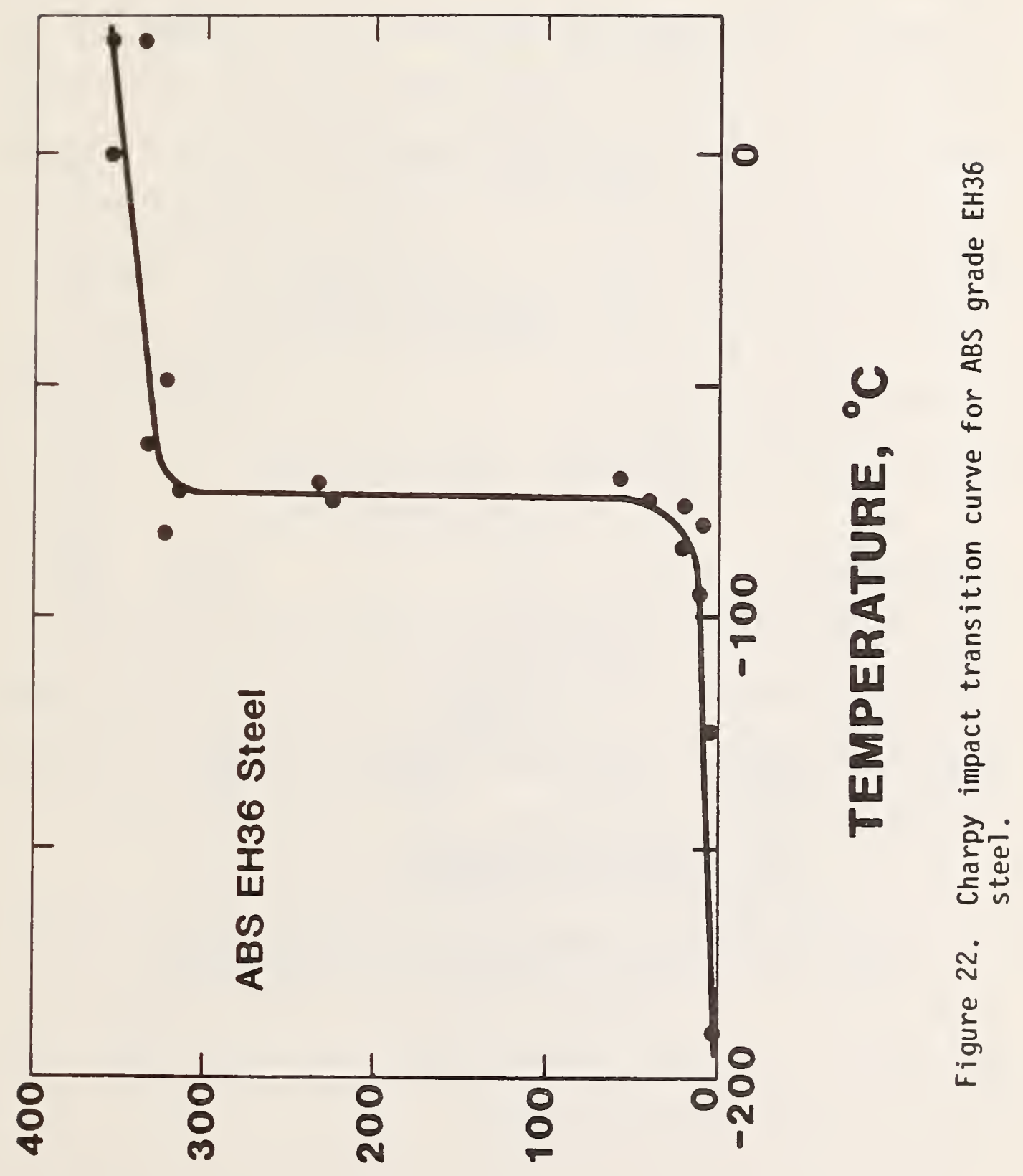

sə|no! ' $\lambda \supset \forall \exists N \exists \perp \supset \forall d W I$ $\Lambda \mathrm{d} \forall \forall H \supset$ 
test machine. Therefore, all energy values above $268 \mathrm{~J}$ do not meet the requirements of ASTM E 23.

\subsection{Fracture Toughness}

The fracture toughness data for the ABS EH36 steel and the pure iron are summarized in Tables 7-9. The critical values of $J$ and CTOD along with the critical fracture event for each specimen are reported. Three critical fracture events have been observed. $J_{c}$ and $\delta_{c}$ are defined at the occurrence of unstable cleavage without prior stable crack growth. $J_{j}$ and $\delta_{i}$ are defined at the onset of stable crack

growth. When cleavage is preceded by stable crack growth $J_{u}$ and $\delta_{u}$ are measured at the point of instability.

\subsubsection{ABS EH36 Steel}

Figures 23 and 24 show that the ductile-to-brittle transition curves shift approximately $30^{\circ} \mathrm{C}$ as the specimen thickness increases from 13 to $38 \mathrm{~mm}$. These data are consistent with previous results [47-55]. It is believed that these upward shifts in the transition are due primarily to increases in crack-tip constraint with thickness. Constraint causes a triaxial stress state and raises the flow stress near the crack tip. This increase in flow stress tends to promote cleavage fracture (i.e., shift the transition curve to the right) since it is easier to reach the fracture stress. 


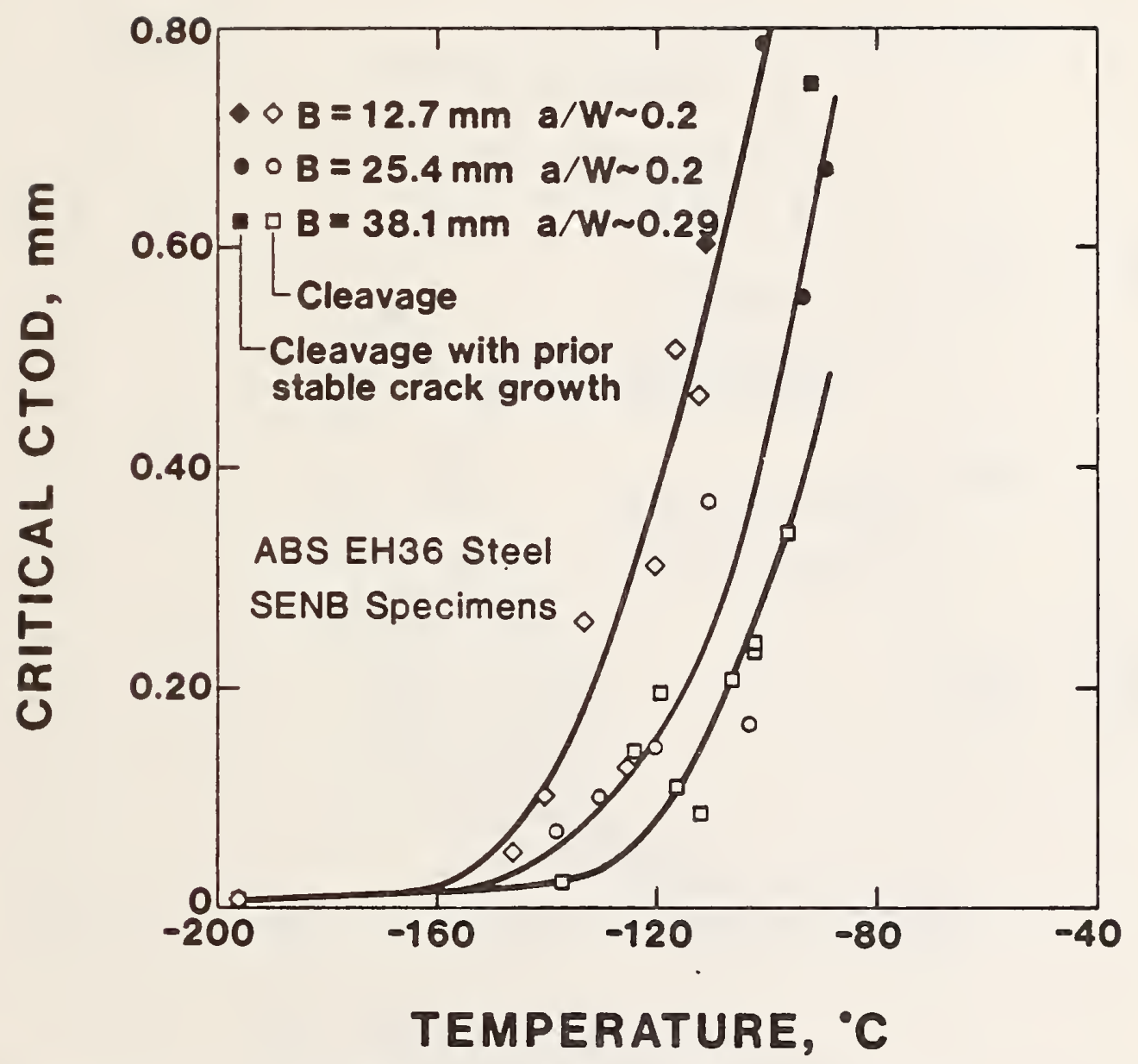

Figure 23. Critical CTOD for cleavage as a function of temperature and specimen thickness for $A B S$ grade EH36 steel. $W=25.4 \mathrm{~mm}(1.0 \mathrm{in})$. 


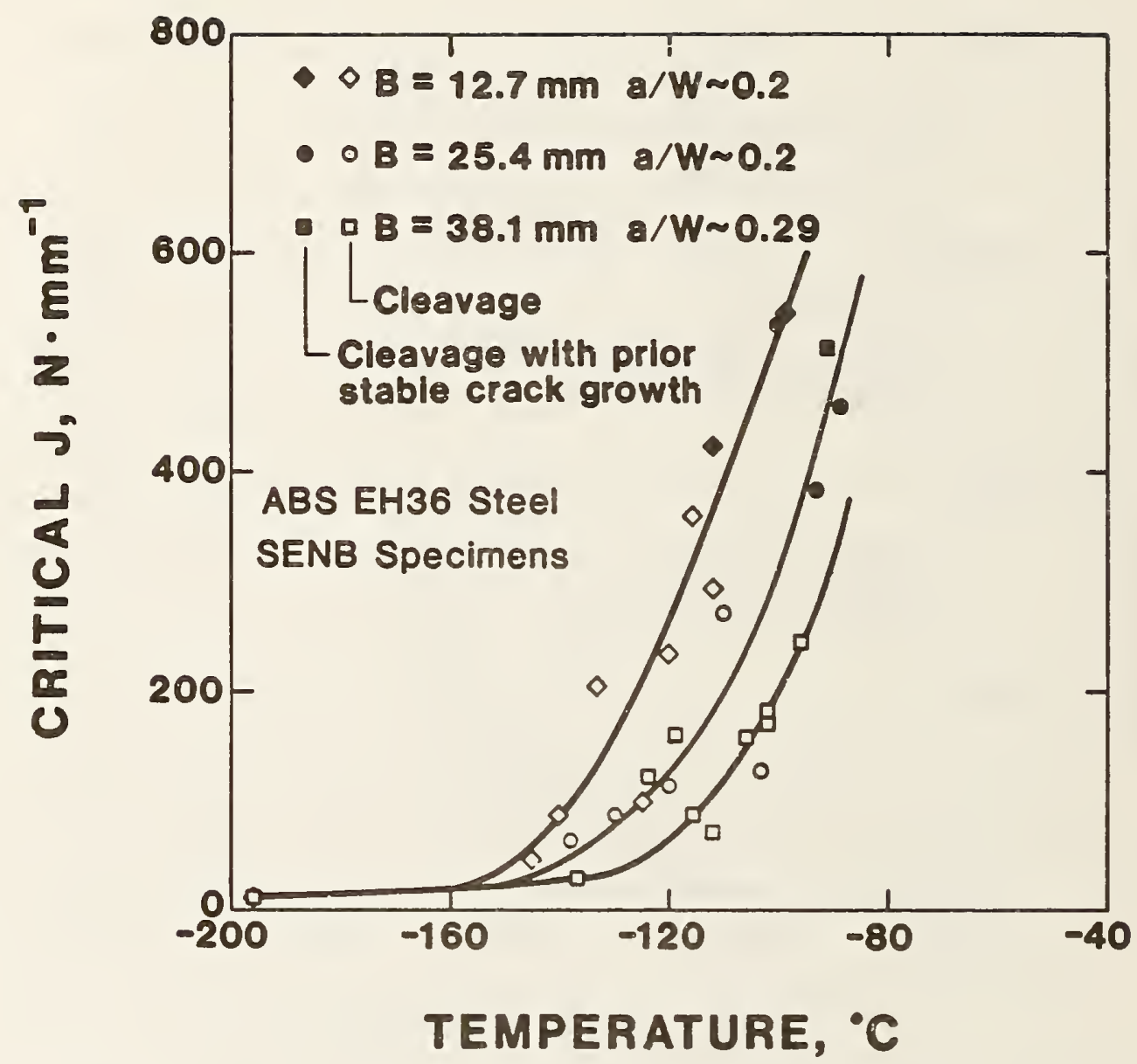

Figure 24. Critical $J$ for cleavage as a function of temperature and specimen thickness for ABS grade EH36 steel. $W=25.4 \mathrm{~mm}(1.0 \mathrm{in})$. 
It should be noted that the thickest specimens $(B=38.1 \mathrm{~mm})$ had slightly deeper cracks than the other two specimen geometries shown in Figures 23 and 24 . The deeper cracks were necessary in order to prevent the load on the thick specimens from exceeding the capacity of the test machine. These deeper cracks probably contributed to the shift in the transition curve to higher temperatures.

The influence of crack length on ductile-to-brittle transition curves is shown in Figures 25 and 26 . The curves in Figures 25 and 26 tend to shift to higher temperatures with increasing crack length. These shifts are due entirely to constraint effects, as opposed to statistical effects. The thickness is constant for these data; thus the volume of material along the crack front is constant.

Deeper-notched specimens (i.e., shorter ligament length) apparently have more crack-tip constraint. The data for the deep-notched geometry (a/W 0.75) behave in a somewhat unusual manner. The curve drawn through these data (Figs. 25 and 26) crosses the curve representing the a/Wn 0.5 data. At low values of $\delta_{C}$ and $J_{C}$, where the plastic zone is relatively sma11, the deep-notched geometry has more constraint than the a/W 0.5 geometry. At higher temperatures (and higher $\delta_{C}$ and $J_{C}$ values) the constraint is apparently relaxed by plastic flow to the nearest free surface.

The effect of ligament length on the slip patterns in SENB specimens has been investigated. Two specimens were coated on one side with a photoelastic material which, when viewed with polarized light, 


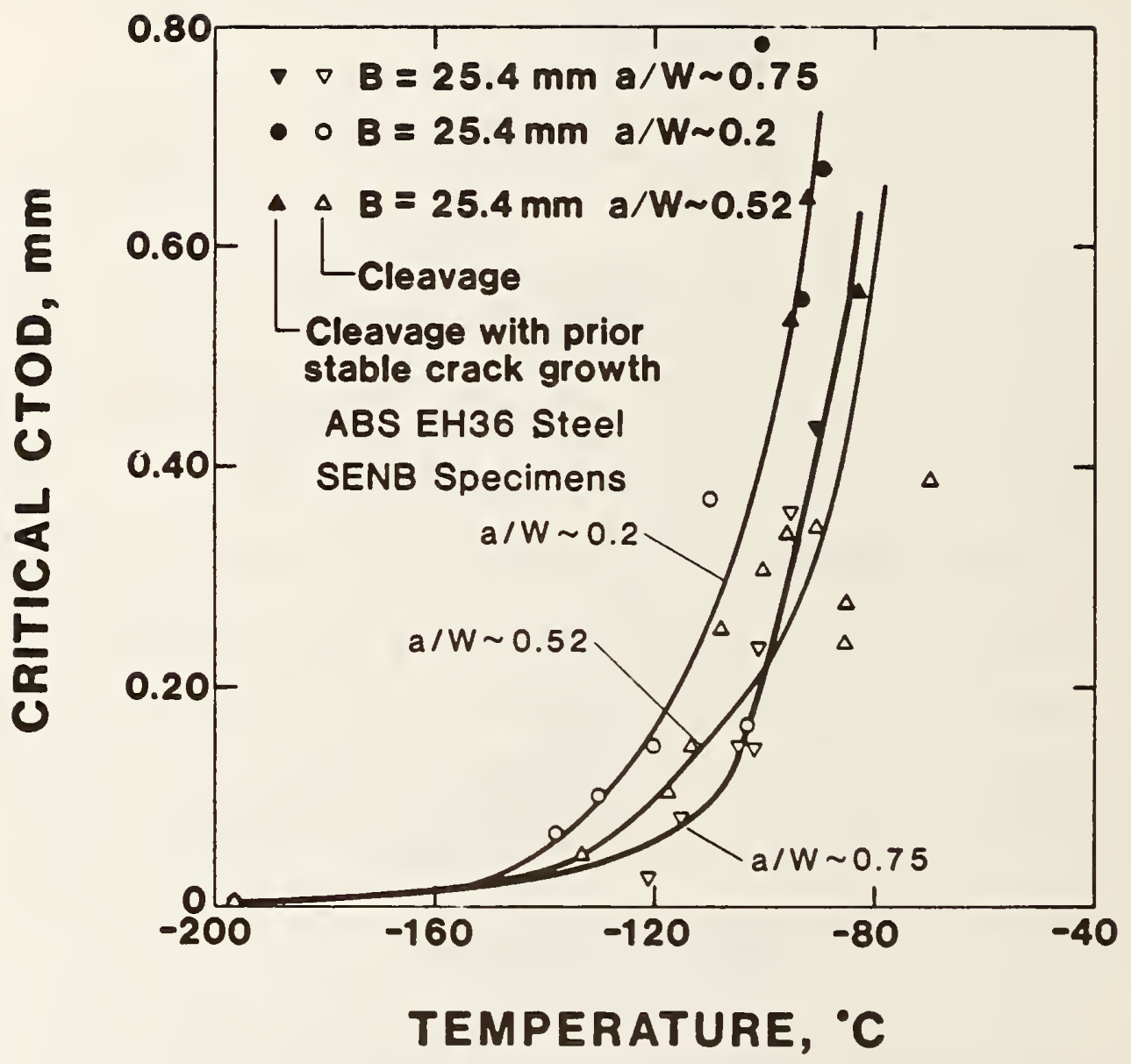

Figure 25. Critical CTOD for cleavage as a function of temperature and crack length for ABS grade EH36 steel. $W=25.4 \mathrm{~mm}(1.0 \mathrm{in})$. 


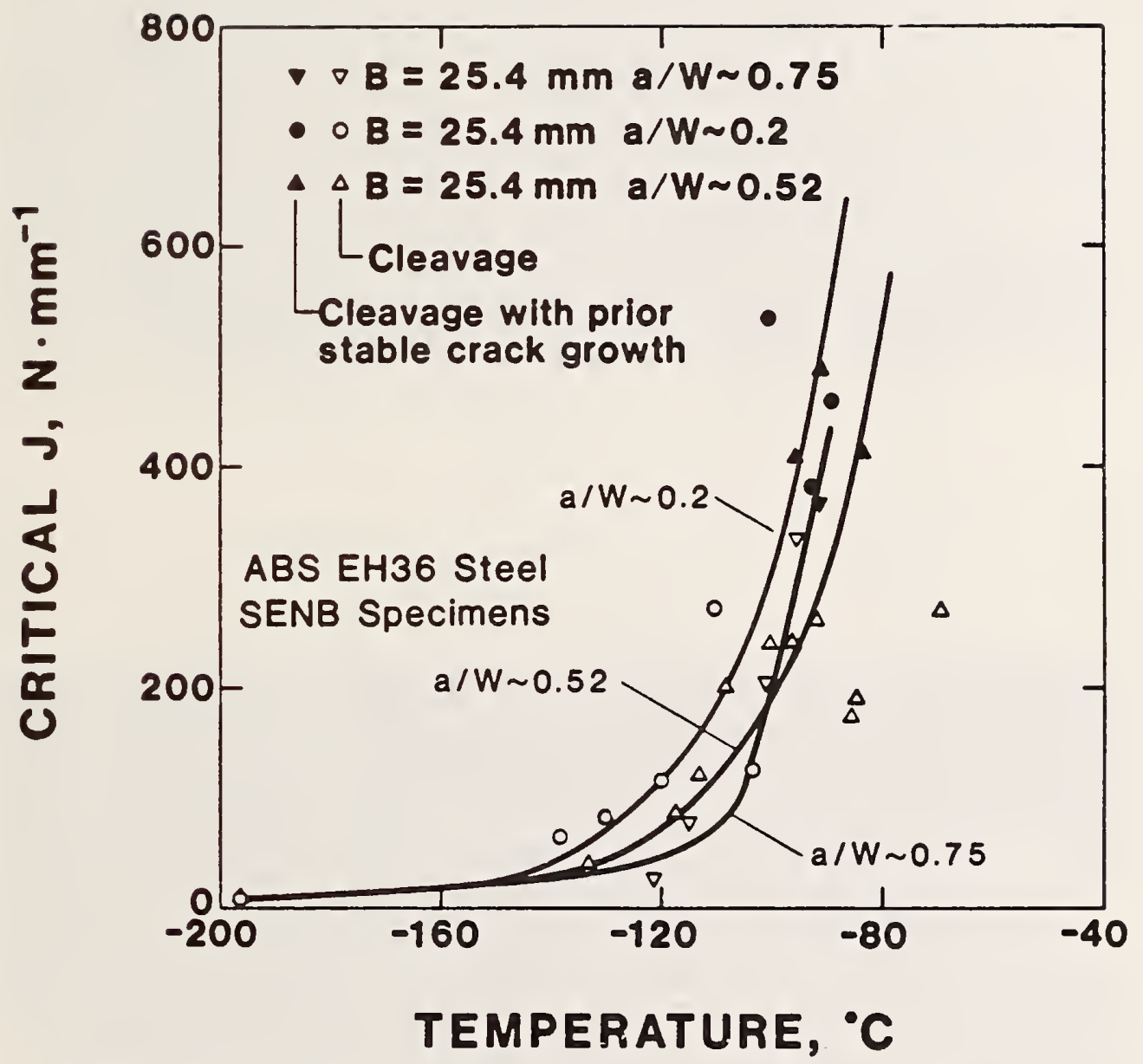

Figure 26. Critical $\mathrm{J}$ for cleavage as a function of temperature and crack length for ABS grade EH36 steel. $W=25.4(1.0 \mathrm{in})$. 
changes color with strain. Figure 27 shows black and white photographs of the multicolor strain patterns on two SENB specimens at net-section yield. The slip lines on both specimens emanate from the crack tip at initial angles of approximately $45^{\circ}$ from the crack plane. These slip lines then deflect inward due to the bending stresses. At the neutral axis the normal stress changes sign; the portion of the ligament below the hinge point is in compression. In the deep-notched specimen (Fig. 27b) the hinge point is closer to the crack tip. The compressive stress field near the crack tip tends to produce additional constraint. As ligament length decreases, the compressive stress field moves closer to the crack tip and constraint increases.

Increasing geometrical constraint causes the load at net-section yield to increase. This elevation of yield load can be quantified by the notch constraint factor, $L$, which is defined by the limit load expression for SENB specimens:

$$
P_{y}=\frac{L \sigma_{0}(W-a)^{2} B}{4 W}
$$

where $P_{y}$ is the load at net-section yield. In the absence of a crack, $L=1$.

Dimensionless load (i.e., load normalized for yield stress and specimen dimensions) is plotted versus mouth-opening displacement in Figures 28 and 29. Dimensionless load is obtained by solving equation (35) for $L$ and allowing $P$ to vary; when $P=P_{y}$, the dimensionless load is equal to $L$. The curves in Figure 28 , which are 


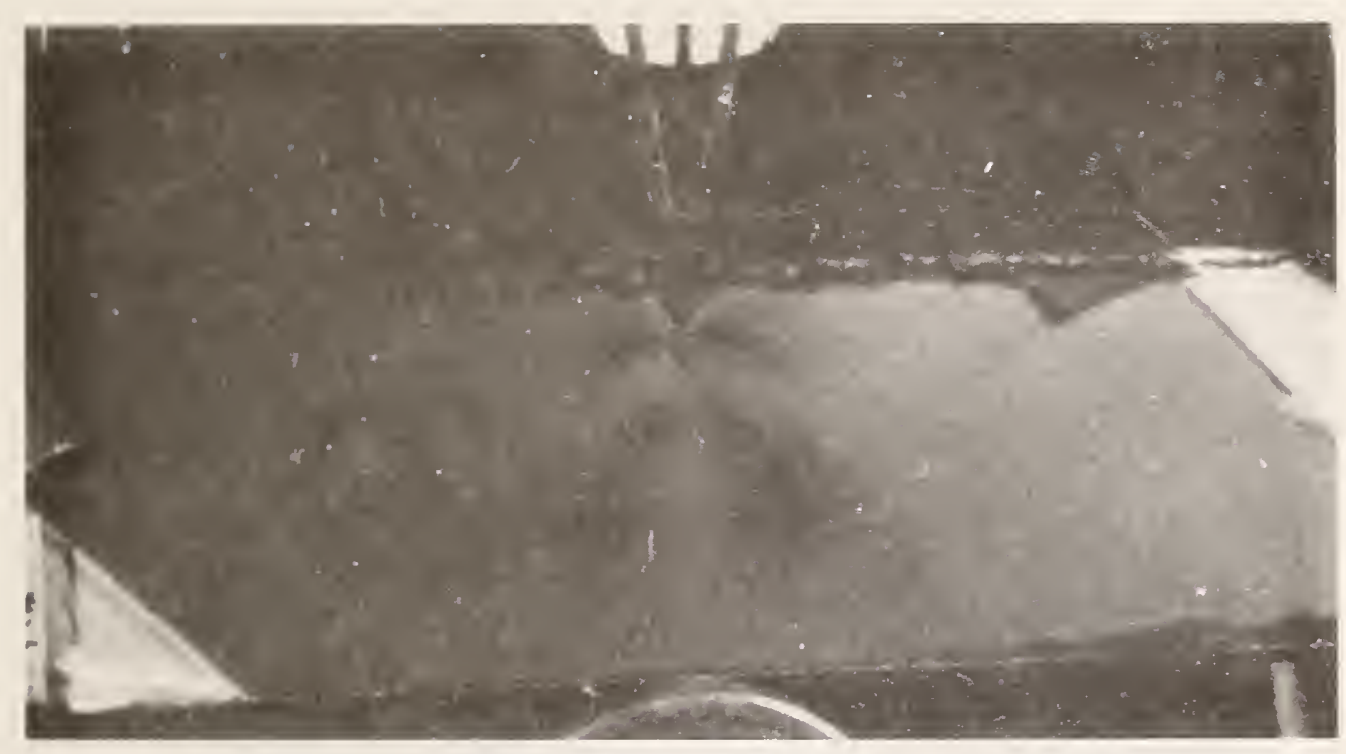

(a)

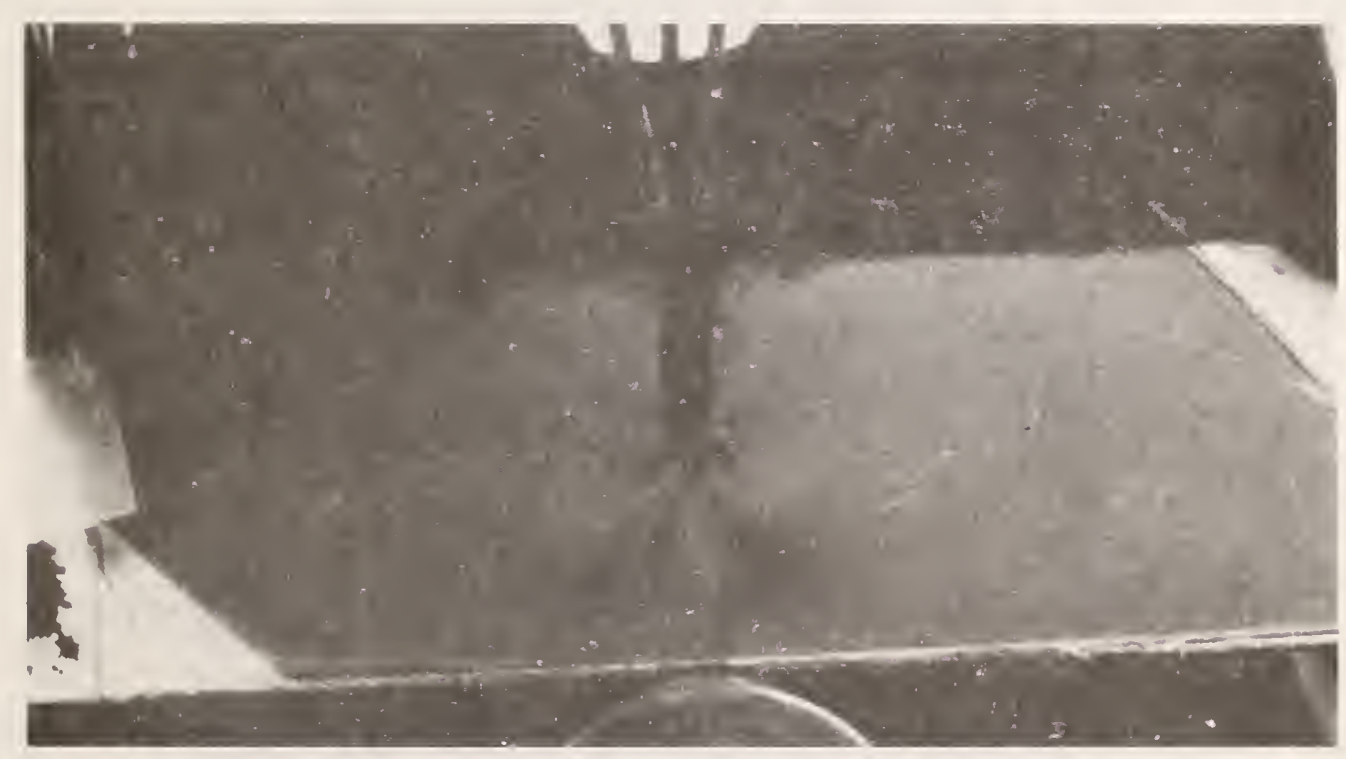

(b)

Figure 27. Photoelastic strain patterns for SENB specimens; a) a/Wn0.2, b) a/WnO.5. 


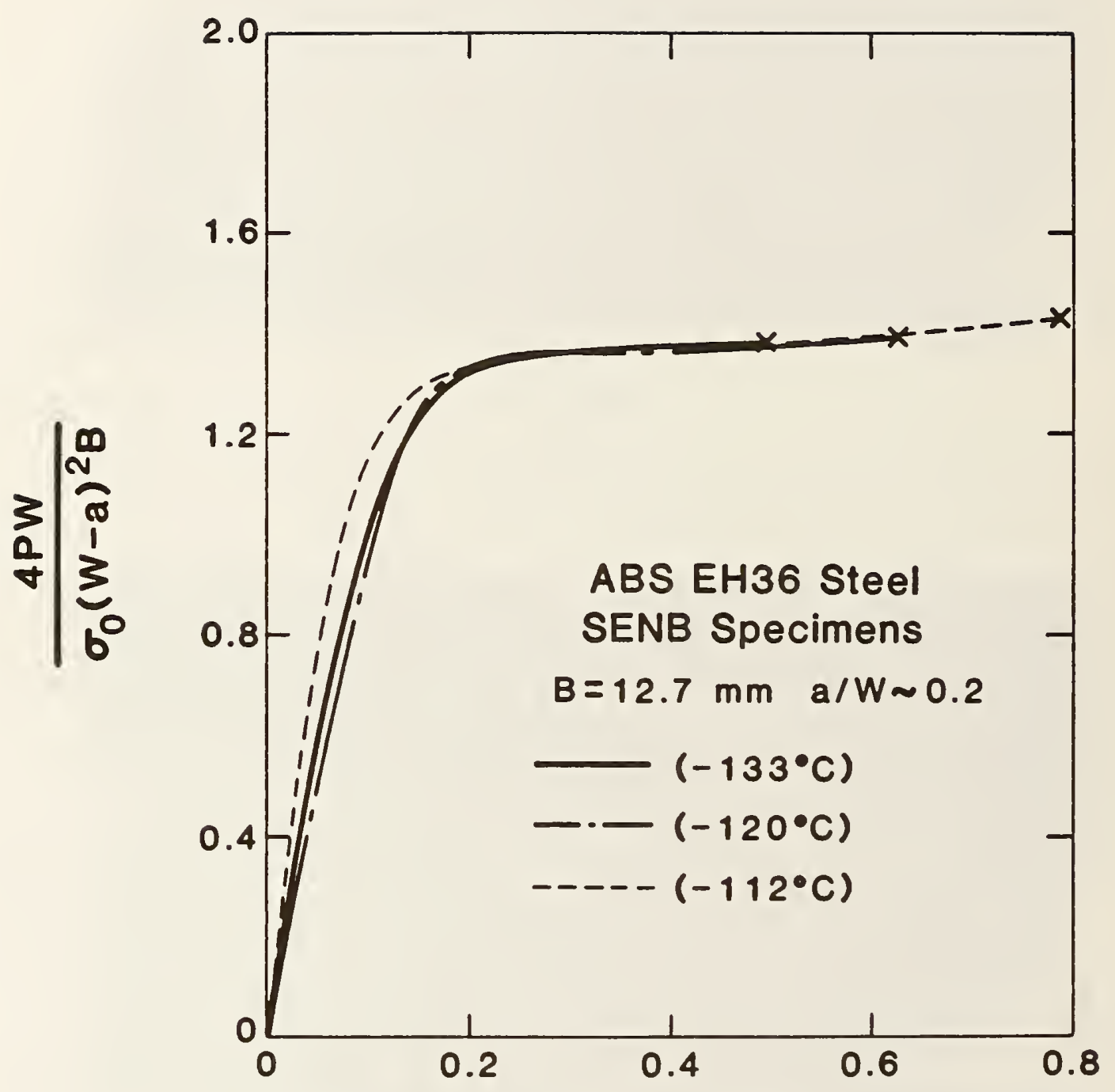

MOUTH-OPENING DISPLACEMENT, mm

Figure 28. Nondimensional-load-versus-displacement curves at various temperatures for constant SENB specimen geometry. 


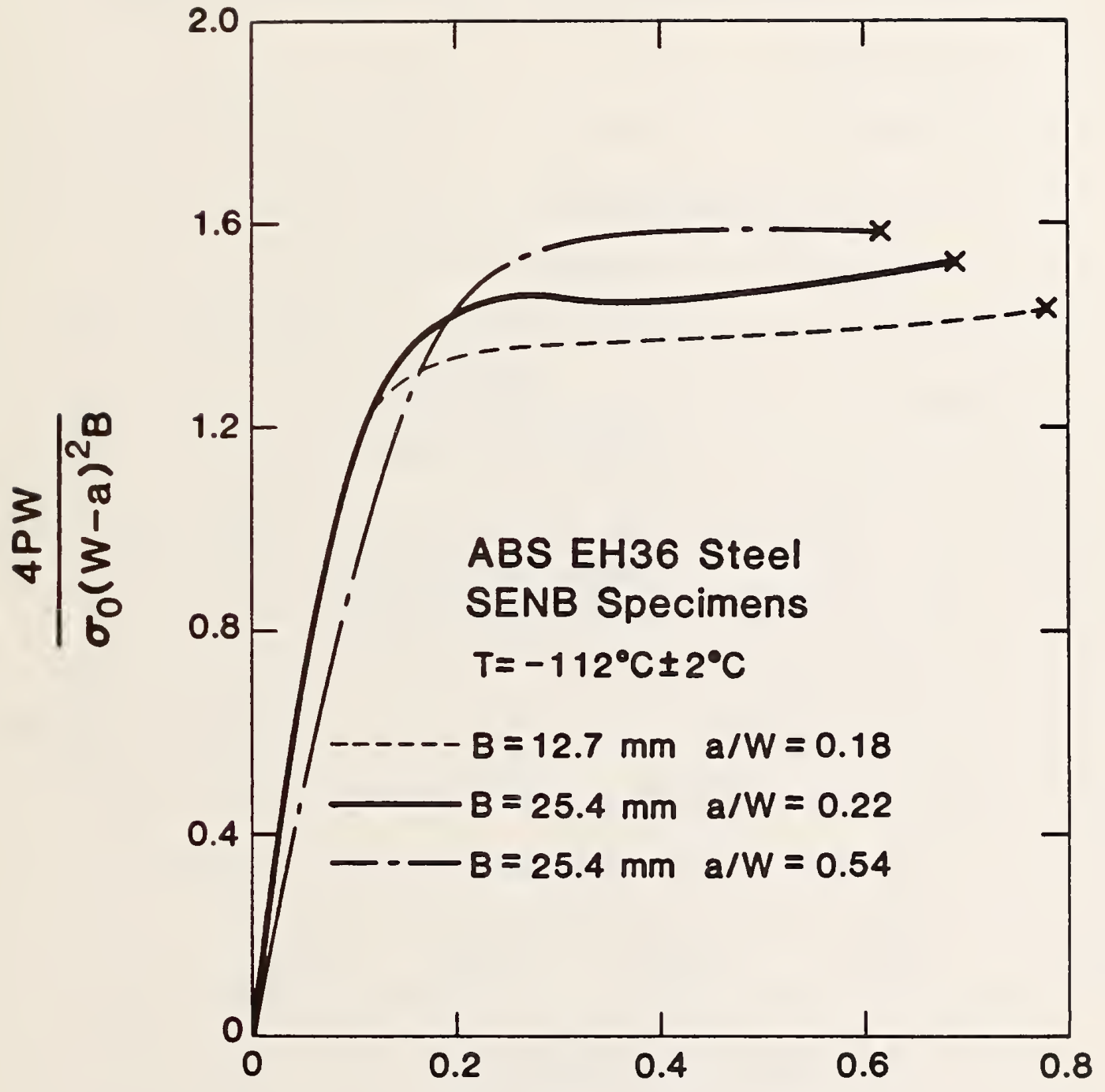

\section{MOUTH-OPENING DISPLACEMENT, $\mathrm{mm}$}

Figure 29. Nondimensional-load-versus-displacement curves as a function of SENB specimen geometry. 
for a constant geometry, form a single curve because load is normalized for yield stress. Figure 29 shows that dimensionless load is elevated when crack length and/or specimen thickness are increased. The curves in Figure 29 are evidence for the hypothesis that the observed shifts in the ductile-to-brittle transition curves (Figs. 23-26) are caused by increases in triaxiality.

Figure 30 shows critical CTOD values for cleavage for $B=25.4 \mathrm{~mm}$ and $B=76.2 \mathrm{~mm}$ (a/W 0.5 for both geometries). Although the scatter is great for these two geometries it is apparent that, in this case, increasing the thickness by a factor of three has little effect on fracture toughness in the transition region. The square-section, a/W 0.5 geometry apparently produces nearly optimum constraint conditions at the crack tip. Constraint in the square-section, a/W 0.2 geometry is not optimal. This is why an increase in thickness by a factor of 1.5 causes a significant shift in the transition curve (see Figs. 23 and 24). At a constant temperature and 1igament length, increasing specimen thickness decreases fracture toughness at a decreasing rate. When constraint is close to maximum (plane strain) further increases in thickness have little or no effect.

The effect of specimen width (W) at a constant ligament length $(W-a)$ is shown in Figure 31. The critical CTOD versus temperature curves represent data from two SENB geometries with nearly identical ligament dimensions. One would expect these two curves to agree since the slip patterns in the ligaments are presumably the same. The actual 


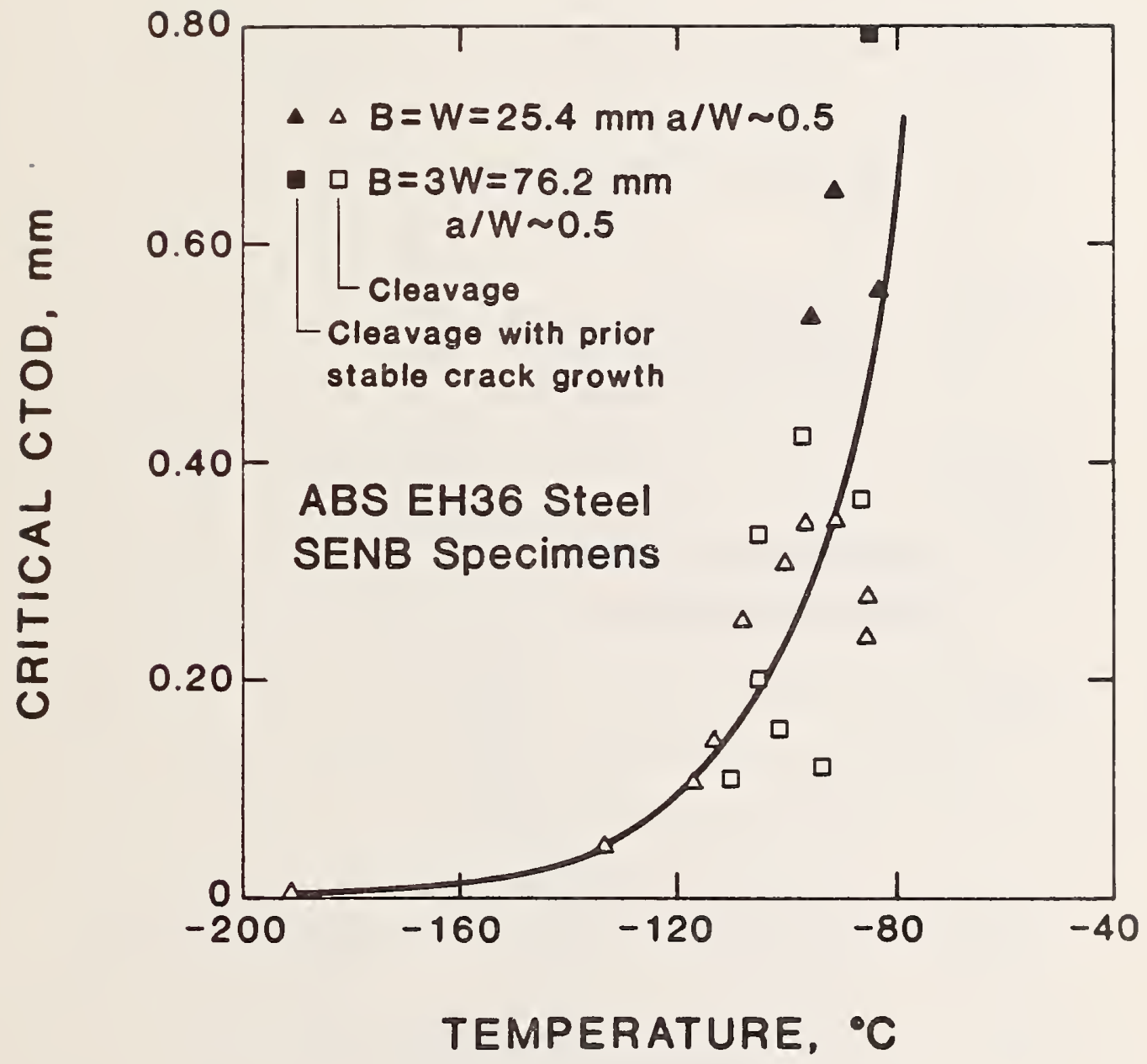

Figure 30. Critical CTOD for cleavage as a function of temperature and thickness for deep-notched SENB specimens of ABS grade EH36 steel. 


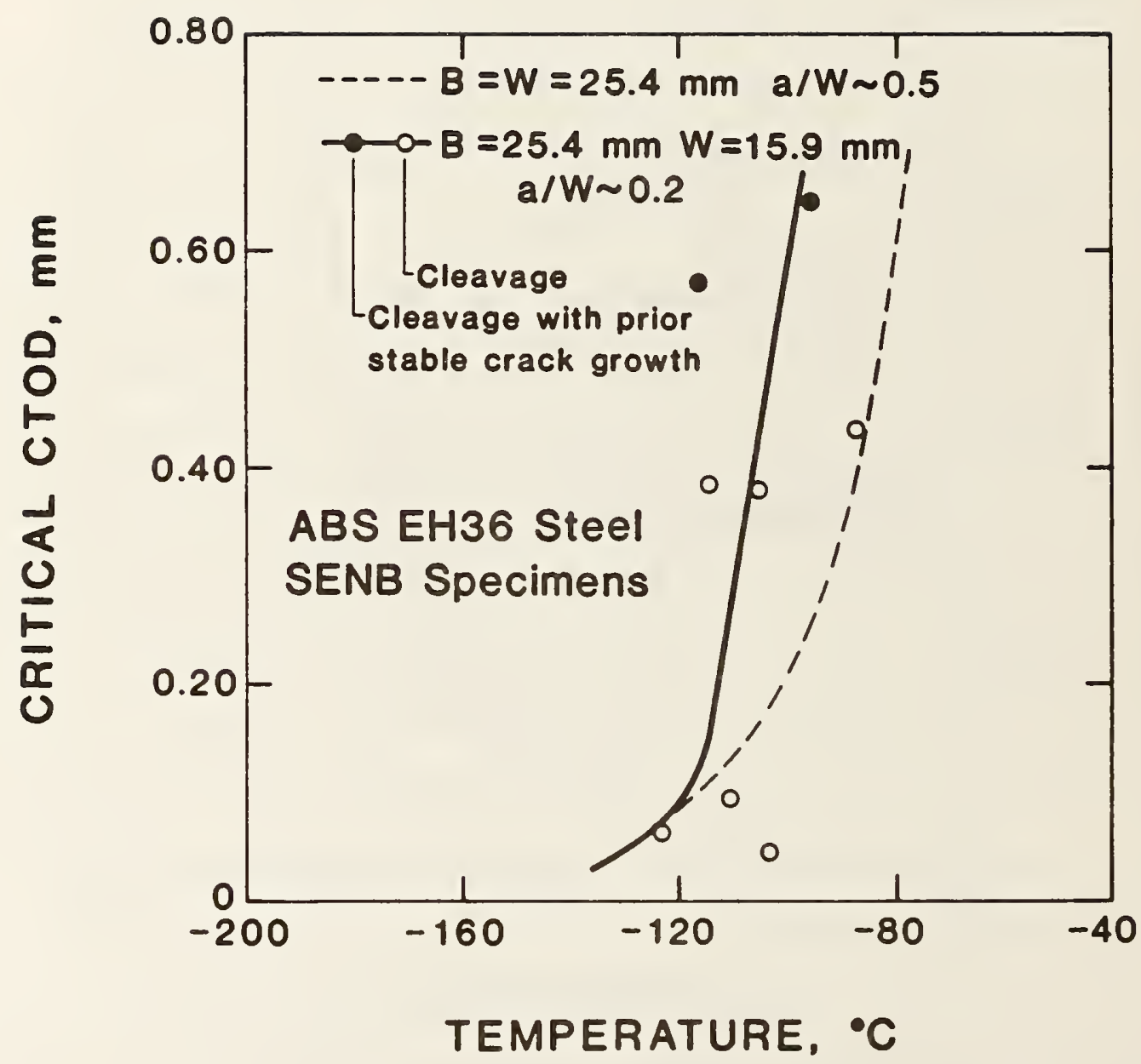

Figure 31. Critical CTOD for cleavage as a function of temperature and specimen width (1 igament length = constant) for ABS grade EH36 steel. 
experimental curves coincide at low toughness values but diverge at high toughness values. The geometry represented by the solid line and data points has a relatively short crack length $(\sim 3 \mathrm{~mm})$. Since the crack tip is near a free surface plastic relaxation to this surface (i.e. gross-section yielding) can occur at high CTOD values. This constraint relaxation due to short cracks causes the transition curve to become steeper. This phenomena is very similar to that observed in Figures 25 and 26 for the deep-notched geometry (a/W 0.75). Apparently, for very short or very deep cracks the constraint is relaxed by plastic deformation from the crack tip to the nearest free surface.

Figure 32 shows critical CTOD values for three geometries of single-edge notched tension (SENT.) specimens. Data for all three geometries fall on approximately the same curve. An increase in thickness from 25 to $76 \mathrm{~mm}$ has little effect on fracture toughness. If smaller thicknesses $(<25 \mathrm{~mm})$ were tested the transition curve would likely shift to lower temperatures. The ligament length has little effect on fracture toughness when the material is loaded in tension. Figure 33 shows the slip pattern of an SENT specimen at net-section yield. The slip lines emanate from the crack tip at $45^{\circ}$ angles on either side of the crack plane. There is no hinge point or stress reversal. The crack tip experiences virtually the same constraint conditions regardless of ligament length. For very short or very deep cracks constraint relaxation would probably occur as in the SENB specimens. 


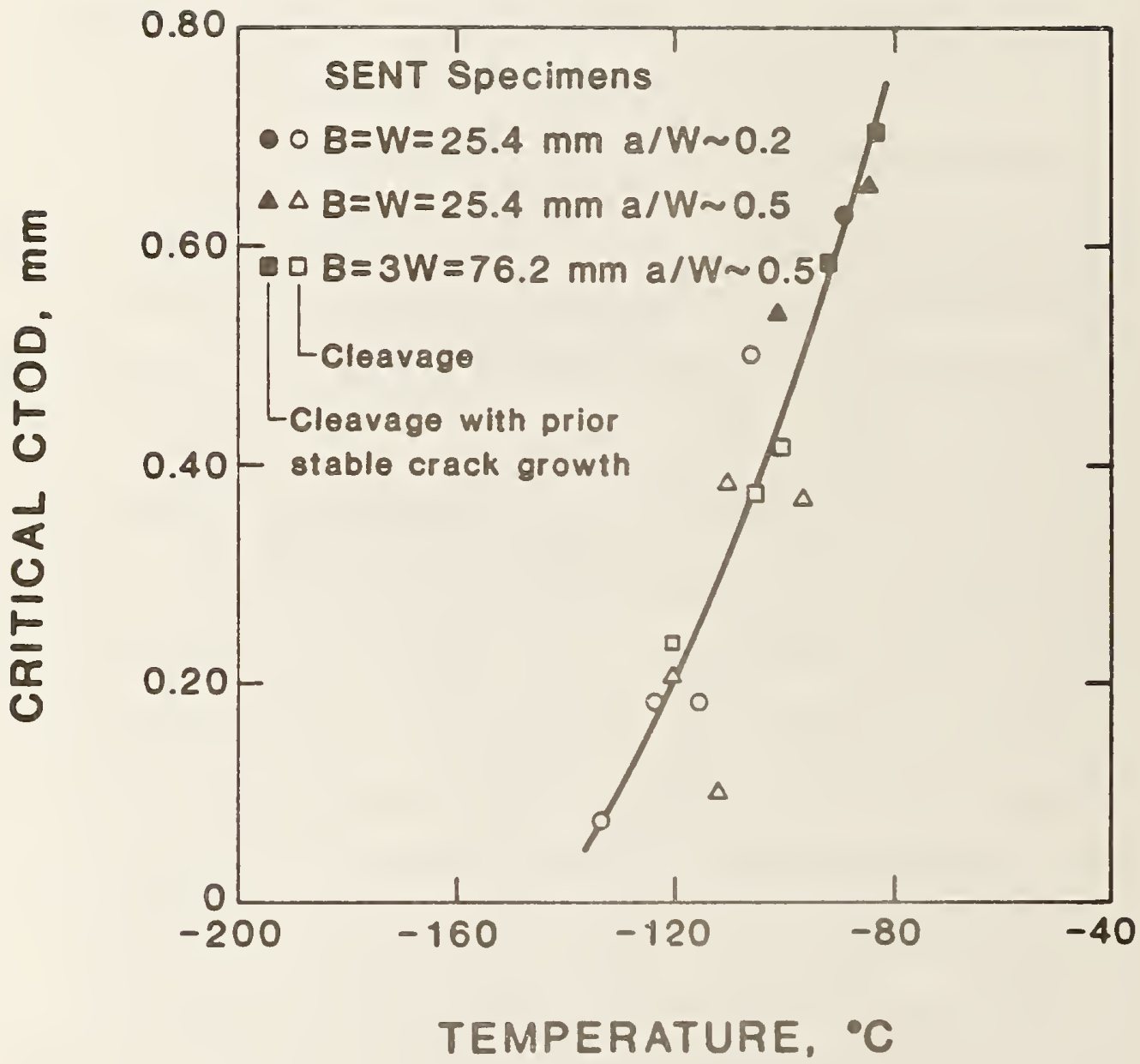

Figure 32. Critical CTOD for cleavage as a function of temperature for three SENT geometries of ABS grade EH36 steel. 


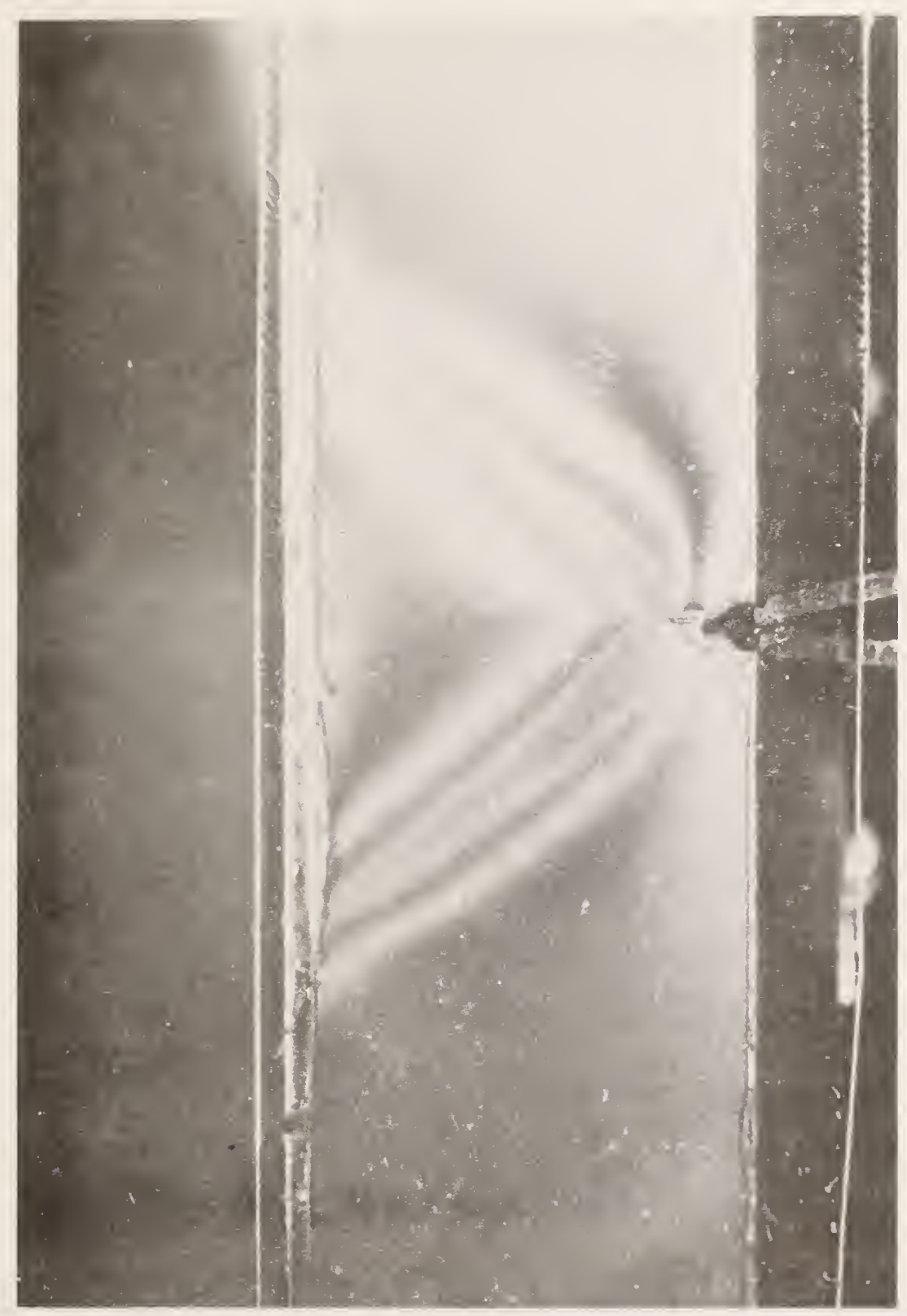

Figure 33. Photoelastic strain pattern of an SENT specimen; a/Wn0.2. 
A comparison of transition curves (critical CTOD vs. temperature) for SENB and SENT specimens with identical crack and ligament dimensions are shown in Figures 34 and 35. Data points were eliminated from these figures for clarity. Figure 35 shows that the bending and tension curves coincide for the square-section, a/W 0.2 geometry. However, Figure 35 shows a definite transition shift between bending and tension for deep-notched (a/W 0.5) specimens. Bending produces an additional increment of constraint in specimens with short ligaments. As the ligament length of an SENB specimen increases the constraint produced by bending decreases and the crack-tip constraint conditions approach those of a tension specimen.

Figures 36 and 37 show values of $\delta_{i}$ and $J_{j}$, respectively, for five SENB specimen geometries of ABS EH36 steel. These data are presented here for the sake of completeness, al though the major theme of this thesis is cleavage fracture. Upper shelf fracture toughness testing is discussed further in Appendix I.

Both $J_{i}$ and $\delta_{j}$ are apparently independent of geometry and $\delta_{i}$ is approximately independent of temperature. $J_{i}$ increases slightly with decreasing temperature because the area under a load-displacement curve at a constant displacement increases with increasing flow stress.

The method for determining these initiation toughness values is given in the appendix. Owing to the uncertainty in this technique the point of incipient crack growth is not well defined, contributing to the scatter seen in Figures 36 and 37. 


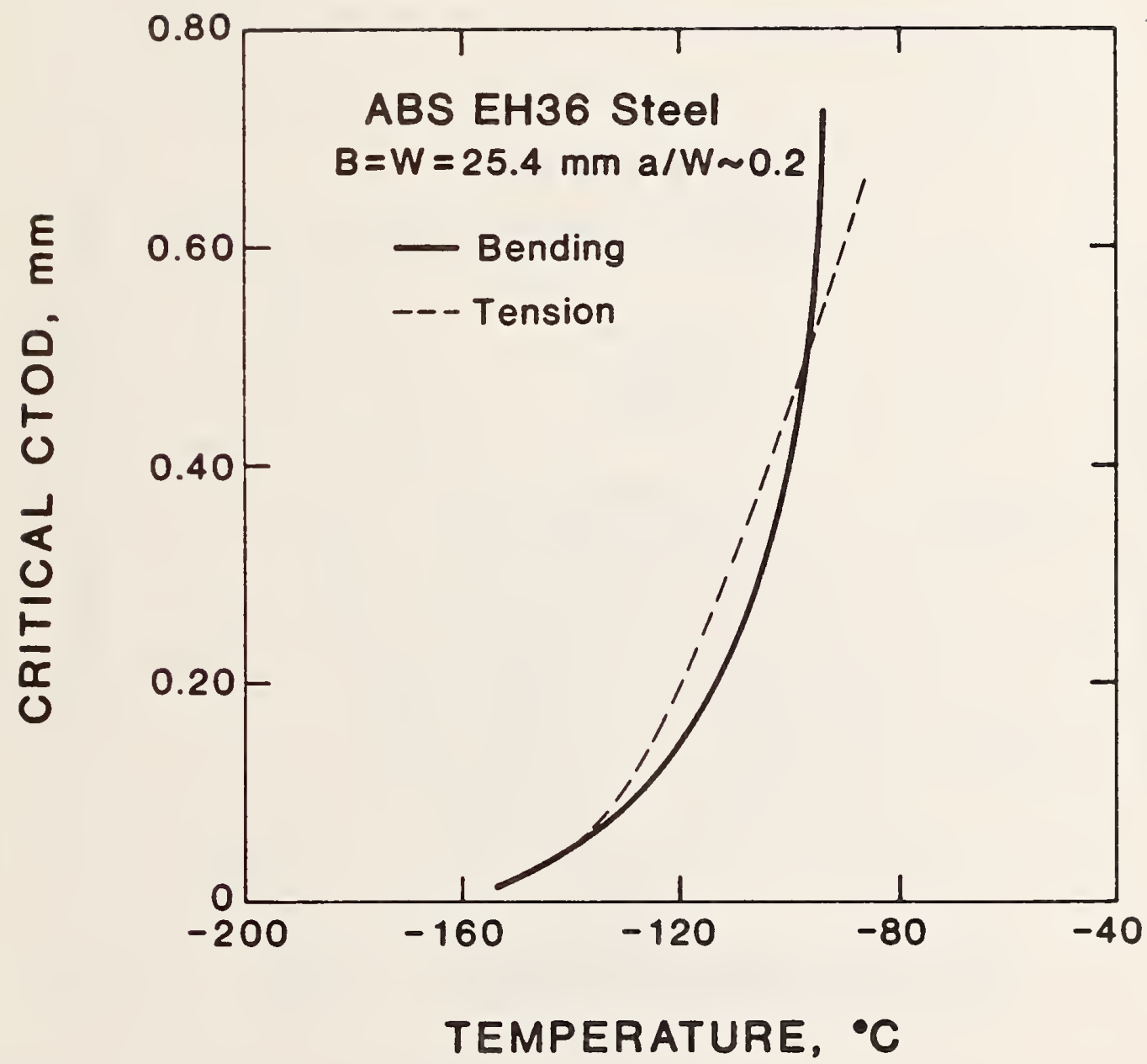

Figure 34. Comparison of bending and tension transition curves for square-section, shallow-notch specimens of ABS grade EH36 steel. 


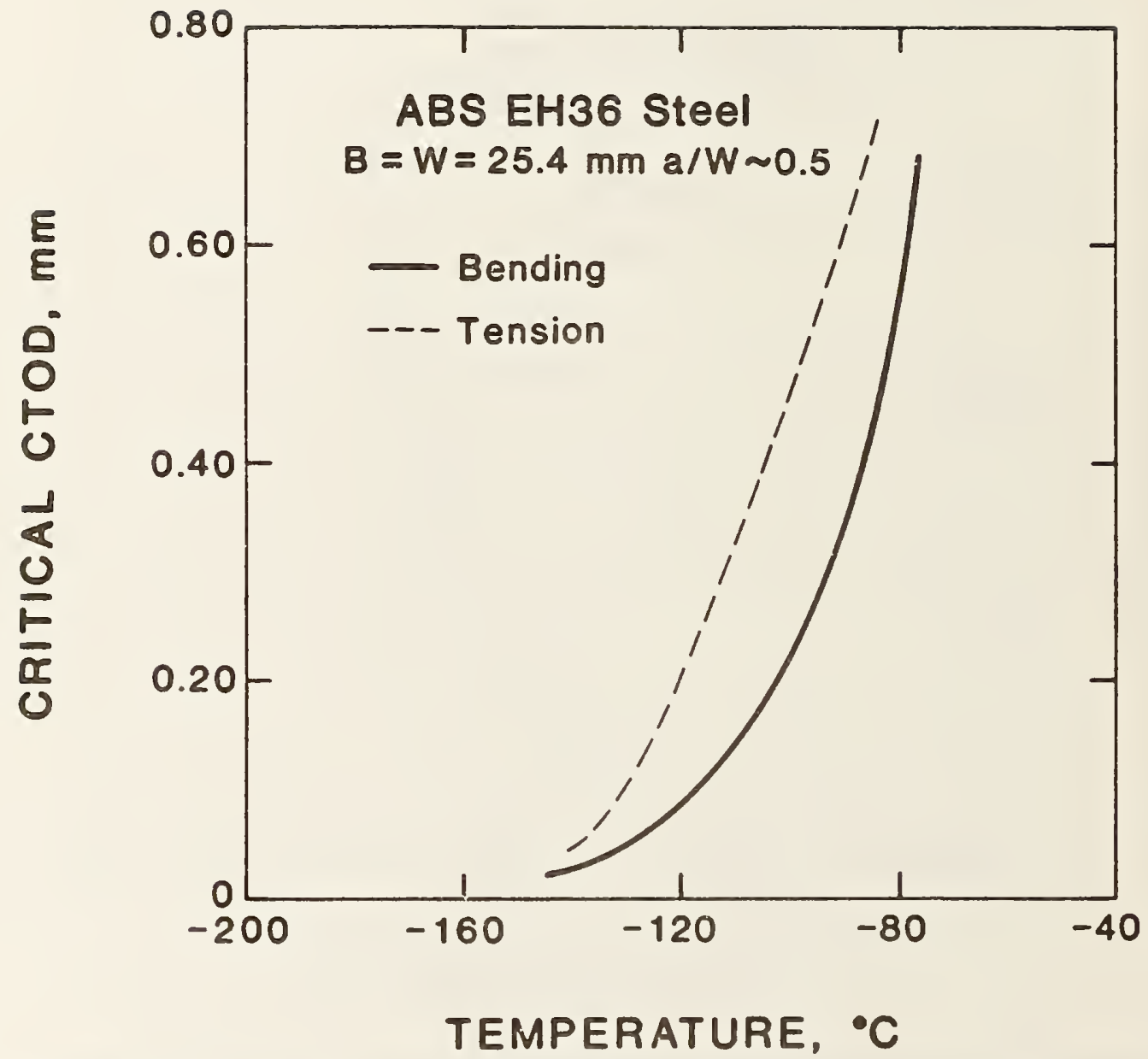

Figure 35. Comparison of bending and tension transition curves for square-section, deep-notch specimens of ABS grade EH36 steel. 


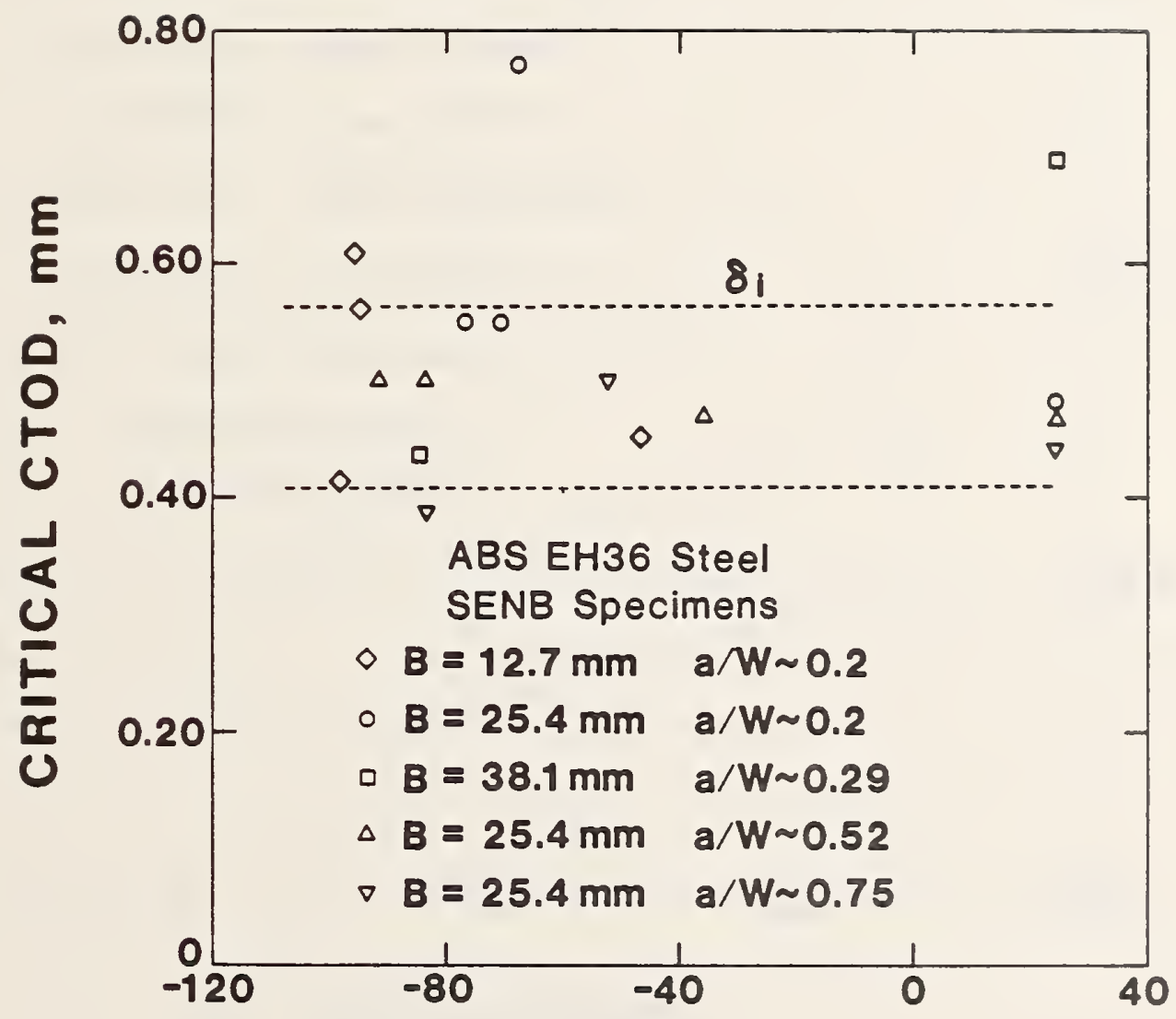

TEMPERATURE, 'C

Figure 36. Critical CTOD for the onset of tearing as a function of temperature and SENB specimen geometry for ABS grade $\mathrm{EH} 36$ steel. $W=25.4 \mathrm{~mm}(1.0 \mathrm{in})$. 


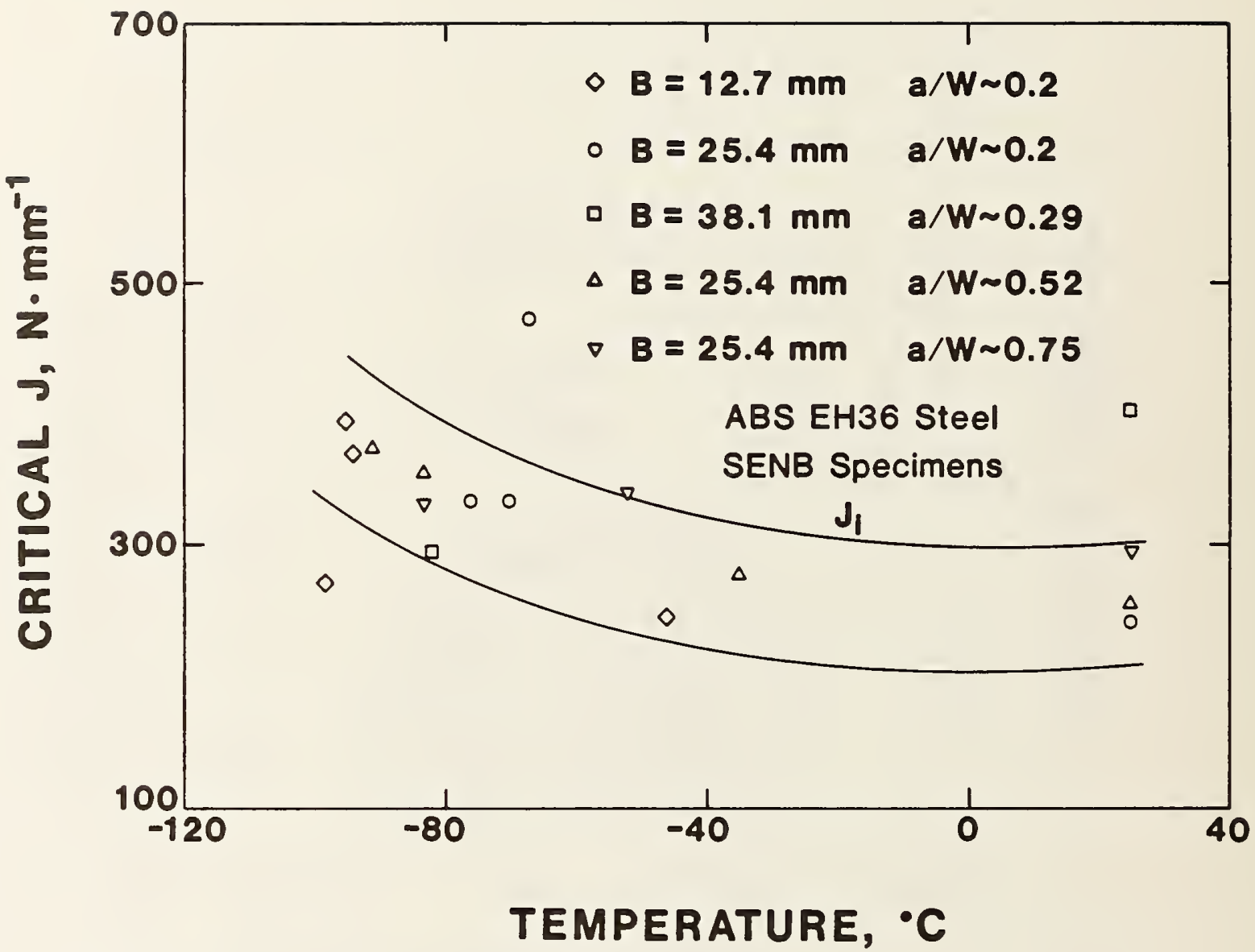

Figure 37. Critical $J$ for the onset of tearing as a function of temperature and SENB specimen geometry for ABS grade EH36 steel. $W=25.4 \mathrm{~mm}(1.0 \mathrm{in})$. 


\subsubsection{Pure Iron}

A 1 imited number of fracture toughness tests were performed on commercially pure iron SENB specimens. The pure iron was chosen as a second material because it has a microstructure that was ideal for the microhardness/strain correlation presented in Section 3.4.1.

The fracture toughness data for the commercially pure iron is shown in Table 9 and Figure 38. A plot of critical CTOD (for cleavage) versus temperature for two thicknesses of SENB specimens is shown in Figure 38. The ductile-to-brittle transition of the pure iron is much steeper than that of the normalized steel. An increase in thickness from $25.4 \mathrm{~mm}$ $(1.0$ in) to $50.8 \mathrm{~mm}(2.0 \mathrm{in})$ results in an upward shift in the transition curve of about $5^{\circ} \mathrm{C}$.

There is a substantial amount of scatter in the data for the $B=50.8 \mathrm{~mm}$ geometry. It is not known why the two square data points on the far right side of Figure 38 do not follow the same trend as the other data for this geometry. However, an examination of the microstructure (Fig. 38b) revealed a number of large inclusions distributed throughout the material. It is possible that the crack tips of the two specimens in question were near inclusions which nucleated large microcracks. This could explain the lower than expected toughness of these specimens. 


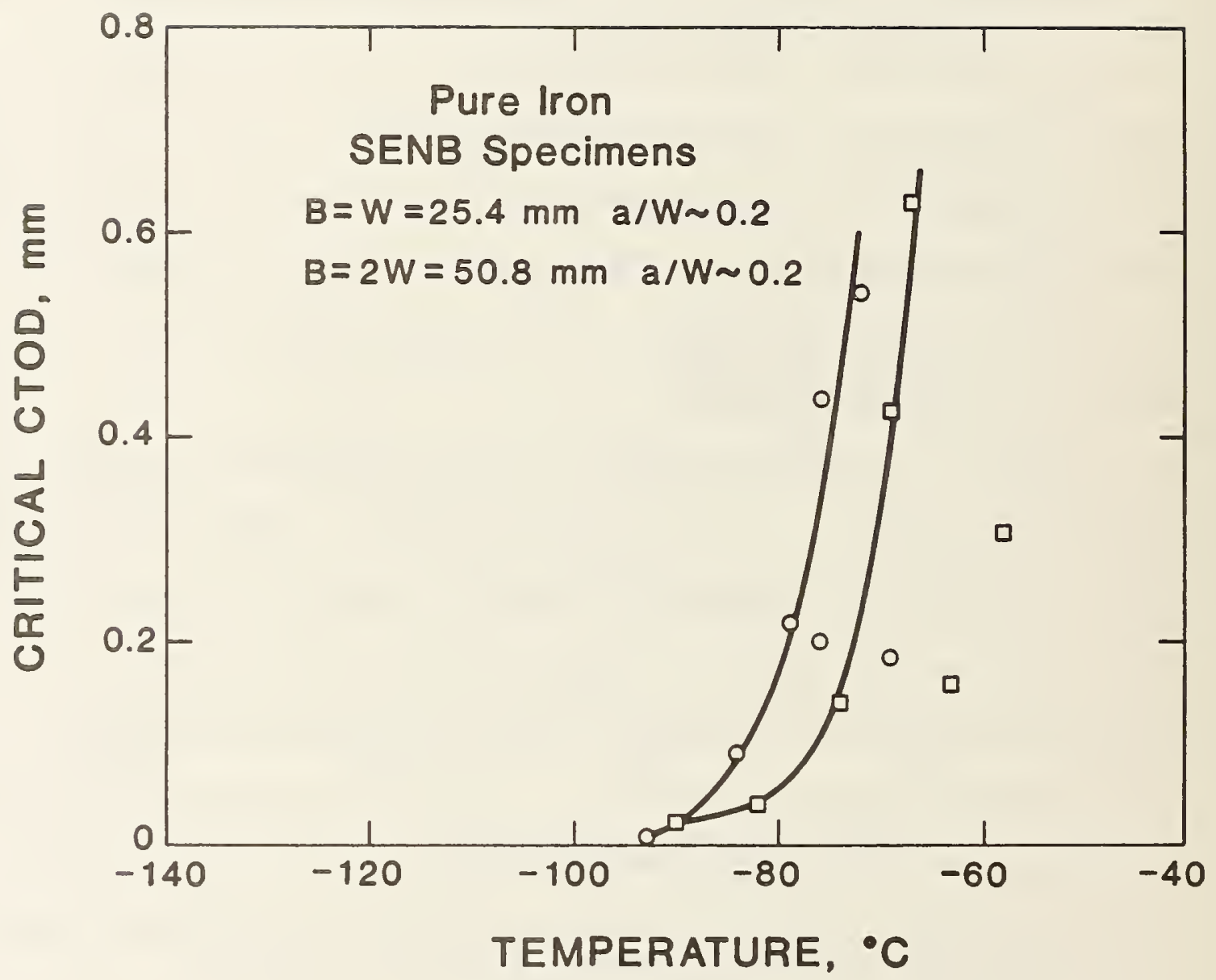

Figure 38. Critical CTOD for cleavage as a function of temperature and thickness for commercially pure iron SENB specimens. 


\subsubsection{Fractography and Metallography}

The microstructures of ABS grade EH36 steel and pure iron are compared in Figure 39. Each micrograph shows a fracture specimen which was sectioned perpendicular to the crack plane after it was deformed to a CTOD of approximately $0.3 \mathrm{~mm}$. The normalized steel has a relatively fine ferrite-pearlite microstructure while the pure iron microstructure consists of very large ferrite grains. As stated above, a number of large inclusions can be seen in the commercially pure iron. According to Tables 1 and 2 the sulfur content of the iron is about three times higher than that of the normalized steel. The crack faces in the normalized steel specimen (Fig. 39a) are relatively straight while the crack faces in the iron are somewhat jagged. This difference in crack profiles is probably due to the coarser grain structure of the iron.

Figures 40 and 41 show scanning electron microscope (SEM) fractographs of the normalized steel and pure iron, respectively. Figures $40 a$ and 41 a show the fracture surfaces of SENB specimens which failed on the lower shelf of toughness. Fracture surfaces of specimens which failed in the ductile-to-brittle transition region are shown in Figures $40 \mathrm{~b}$ and $41 \mathrm{~b}$. The major difference between lower-shelf and transition behavior as seen by fractography is the presence of a stretch zone on the transition region fracture surfaces. Lower-shelf fractures occur when the crack is relatively sharp while in the transition region, fracture occurs after significant blunting of the crack tip. Transition and lower-shelf fracture surfaces both contain flat, smooth cleavage 


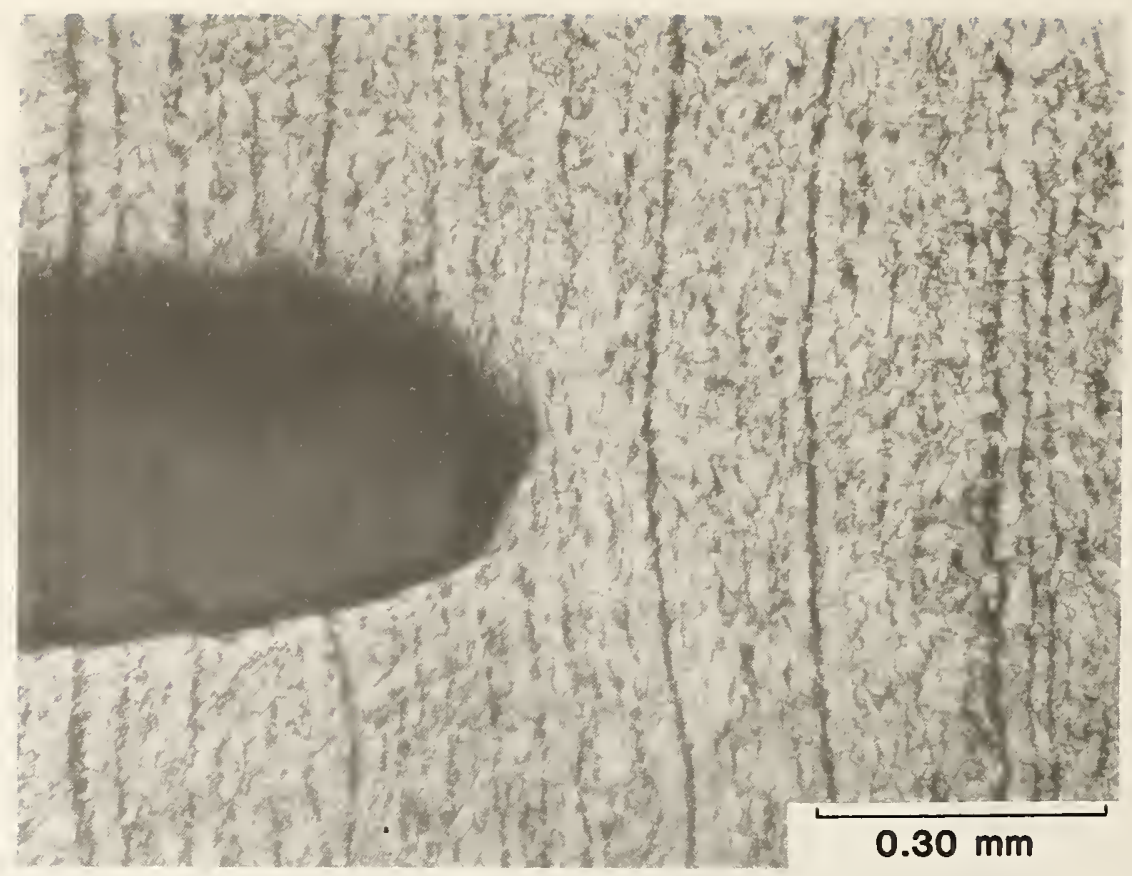

(a)

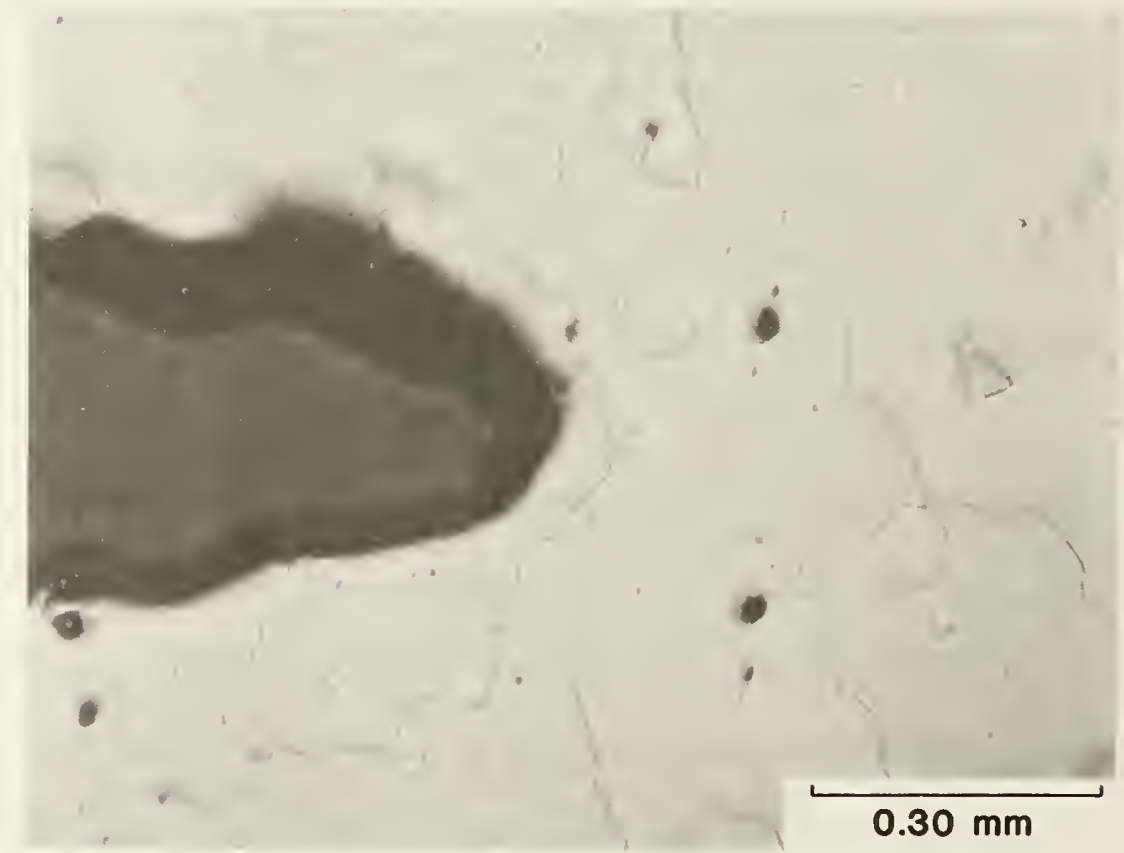

(b)

Figure 39. Photomicrographs of blunted crack tips which show the microstructures of a) ABS grade EH36 steel, and b) commercially pure iron. Magnification $=100 x$. 


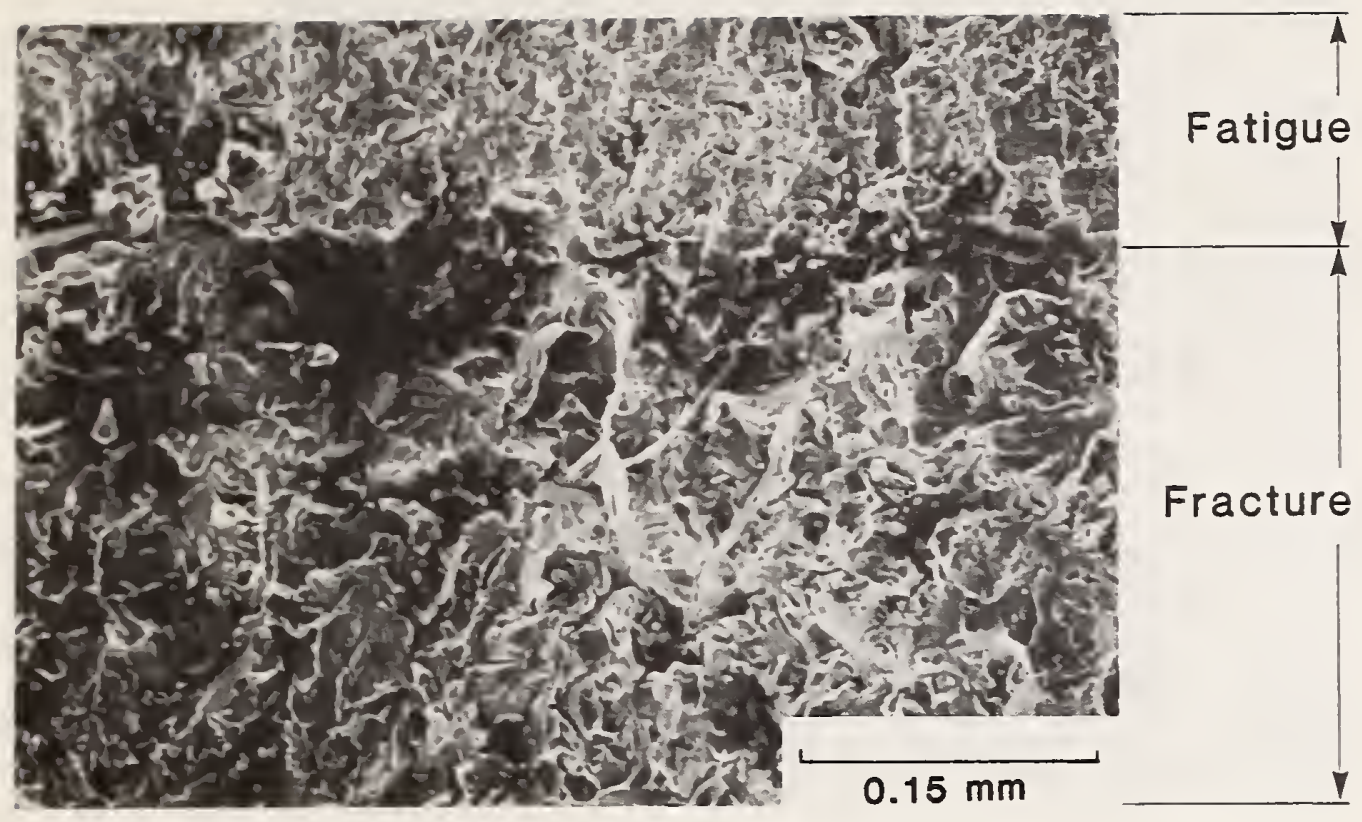

(a)

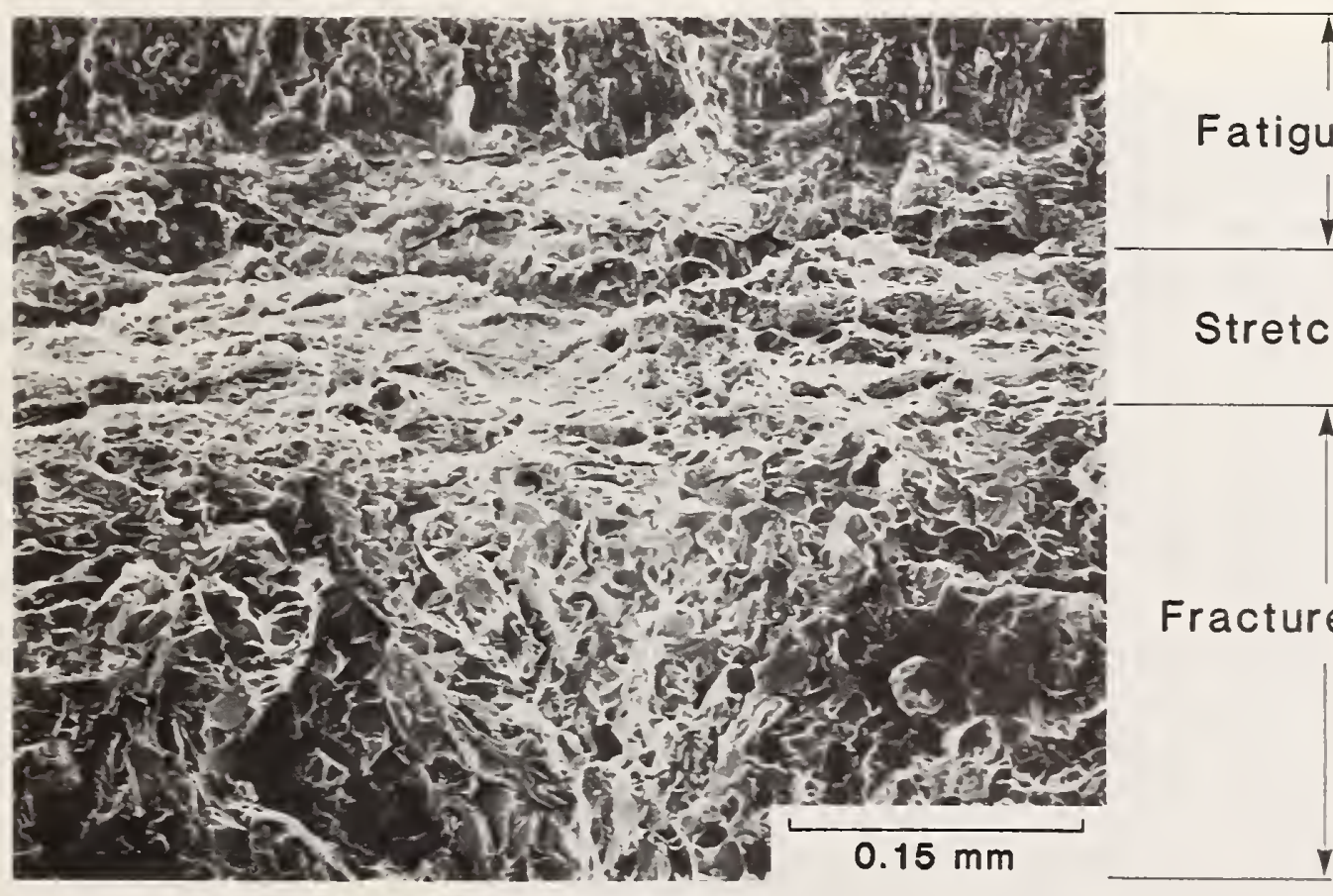

(b)

Figure 40. SEM fractographs of ABS grade EH36 steel which compare lower-shelf and transition region fracture surfaces. a) SENB spec $B-9, \delta=0.004 \mathrm{~mm}$; b) SENB spec B-11, $\delta_{c}=0.371 \mathrm{~mm}$. Magnification $=200 x$. 


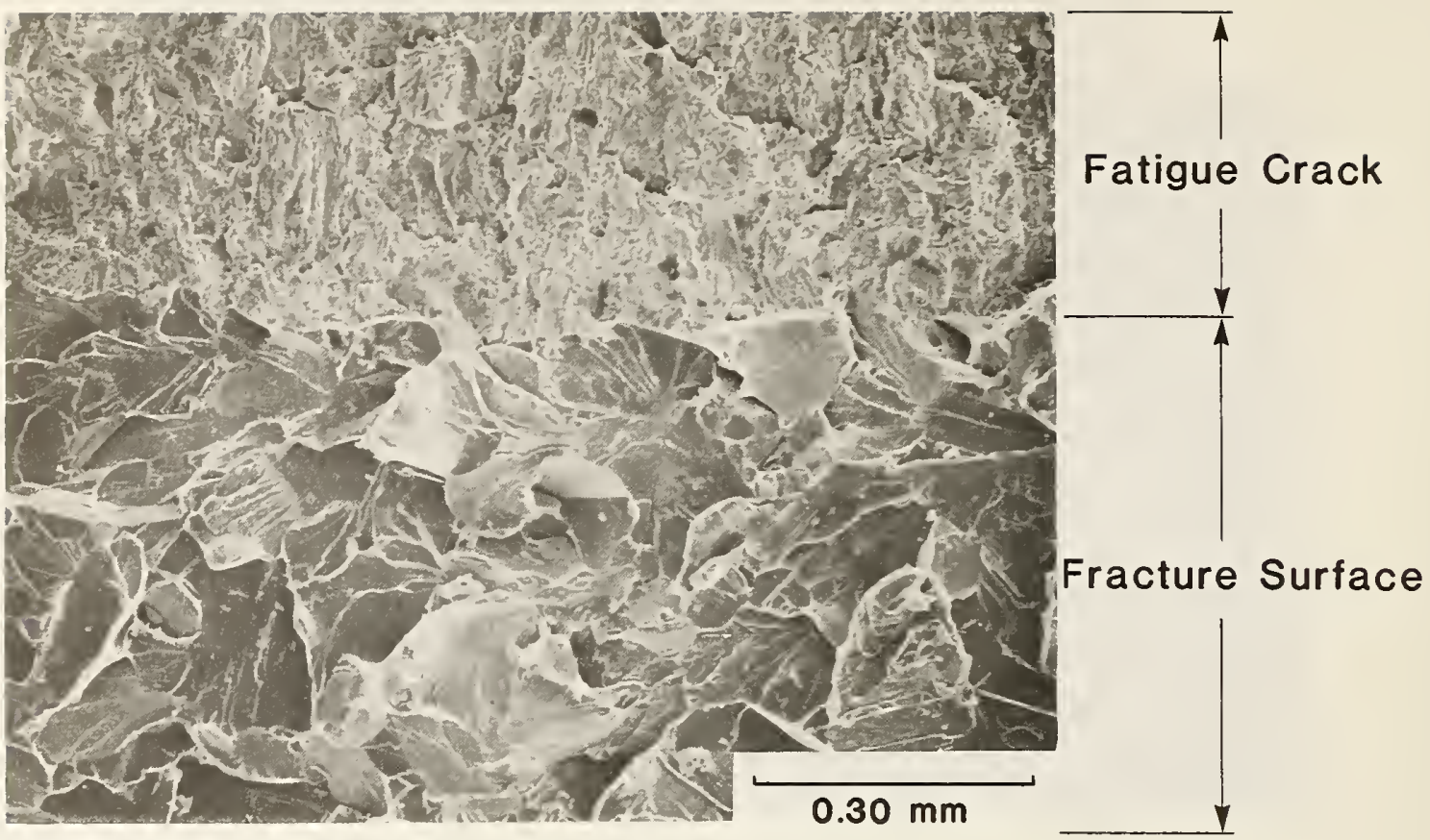

(a)
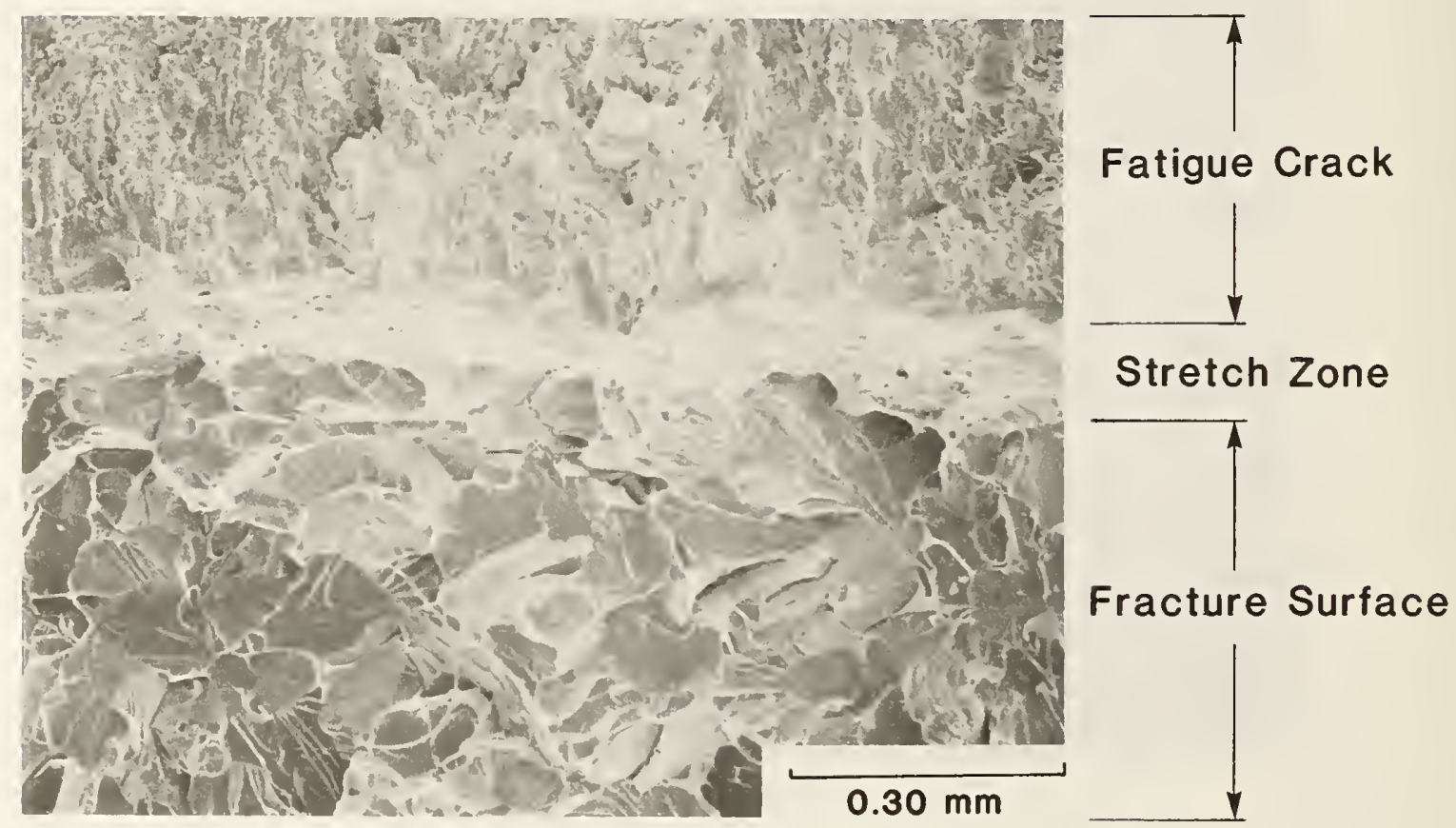

(b)

Figure 41. SEM fractographs of pure iron which compare lower-shelf and transition region fracture surfaces. a) Spec J-4, $\delta=0.010 \mathrm{~mm}$; b) spec. J-7, $\delta_{c}=0.540 \mathrm{~mm}$. Magnification $=100 x$. 
facets with "river pattern" lines. These "river patterns" are more visible in the pure iron fractographs because the facets are much larger than those of the steel.

Figures 42 and 43 show high magnification fractographs of the stretch zones in both materials. There is some ductile tearing in the stretch zone of the steel specimen (Fig. 42) as evidenced by the numerous microvoids which have coalesced. One can see spherical particles (presumably sulfide inclusions) at the bottom of some of the voids in Figure 42b. The crack in the iron specimen (Fig. 43) did not tear prior to fracture. However, a high degree of plastic deformation has occurred in the stretch zone and a number of voids have formed.

The fracture surface of a normalized steel specimen is compared to that of a iron specimen in Figure 44 . The cleavage facets on the iron fracture surface are almost an order of magnitude larger than the facets on the steel specimen. This is to be expected from an observation of the respective microstructures (Fig. 39) since facet size is related to grain size. The river patterns can be clearly seen in both fractographs. Some secondary cracking can also be seen, especially in the iron (Fig. 44b). Several small cracks in the far left portion of Figure $44 b$ are parallel to each other; i.e., they occur on the same crystallographic plane.

The fractographs in Figures $40-44$ refute a common misconception about the ductile-to-brittle transition. It is sometimes said that fracture on the lower shelf occurs by cleavage while fracture in the 


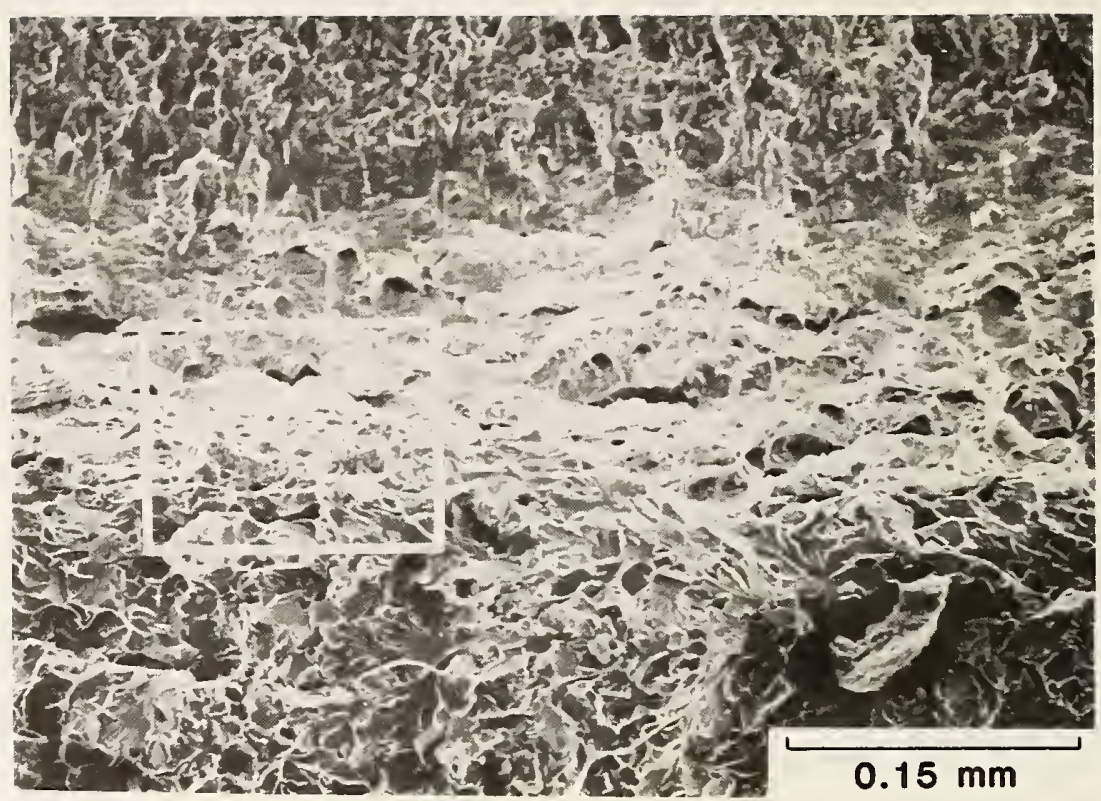

(a)

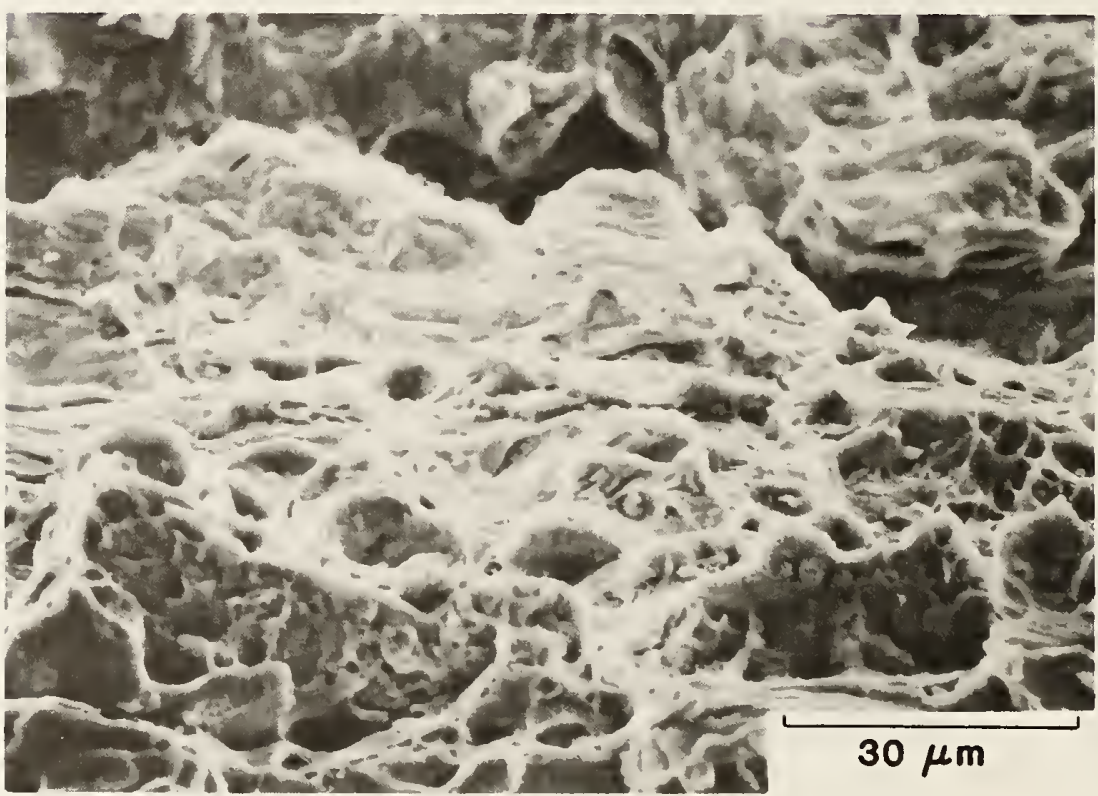

(b)

Figure 42. SEM fractographs of ABS EH36 specimen B-11 which show a magnified view of the stretch zone. a) $200 x$; b) $1000 x$. 


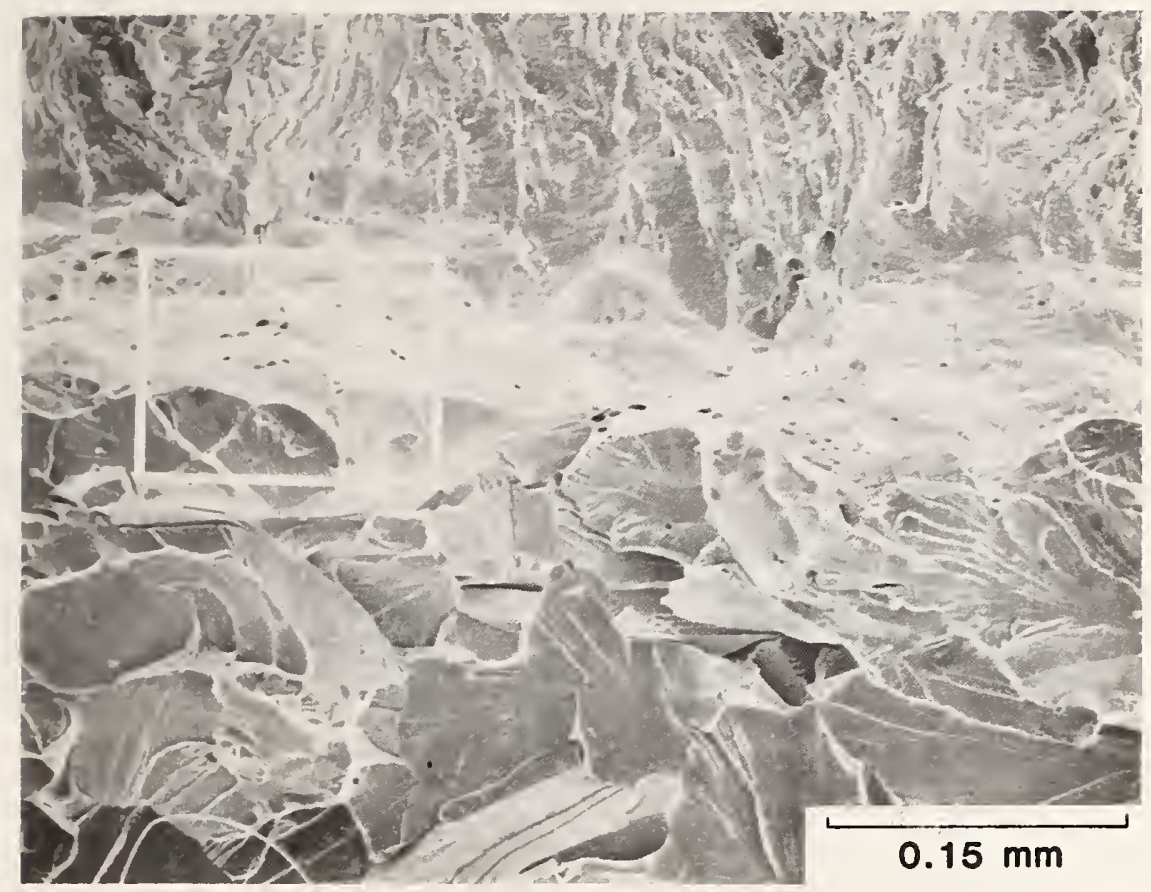

(a)

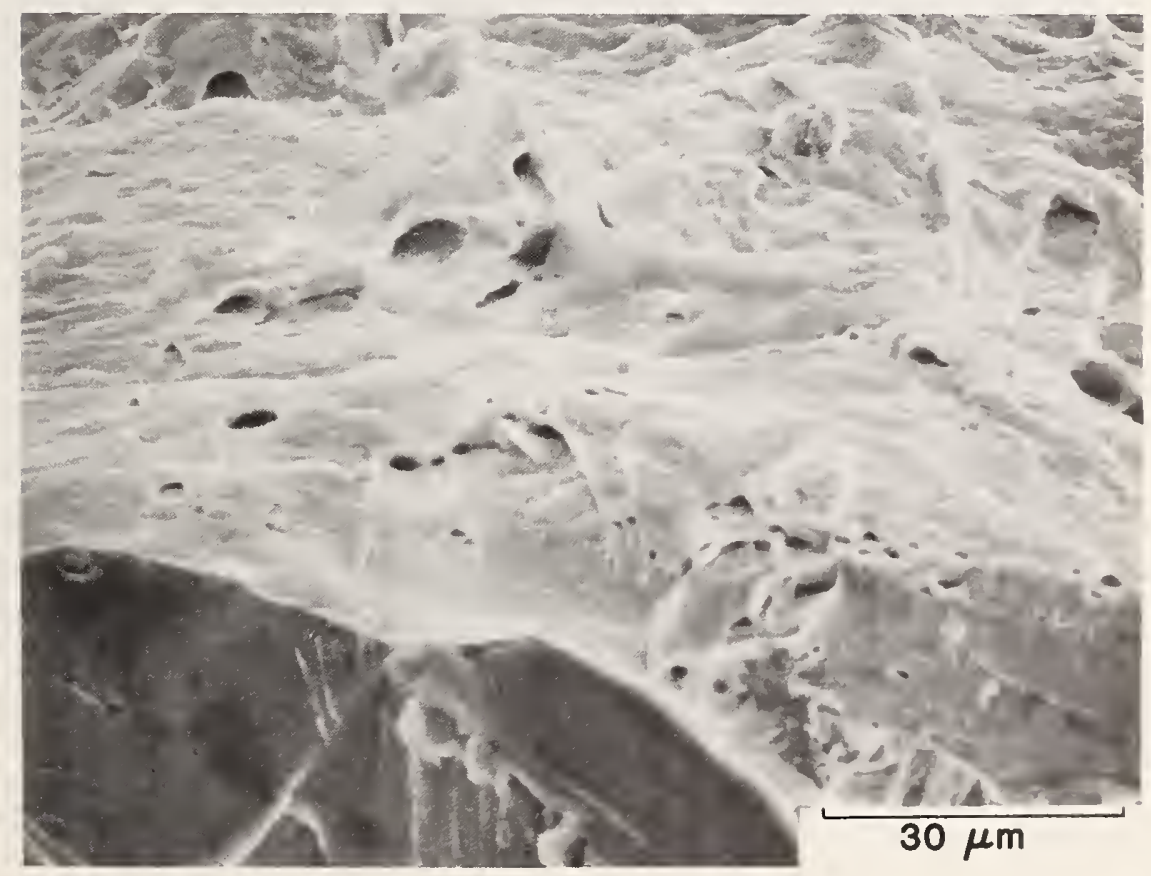

(b)

Figure 43. SEM fractographs of pure iron specimen J-7 which show a magnified view of the stretch zone. a) 200x; b) 1000x. 


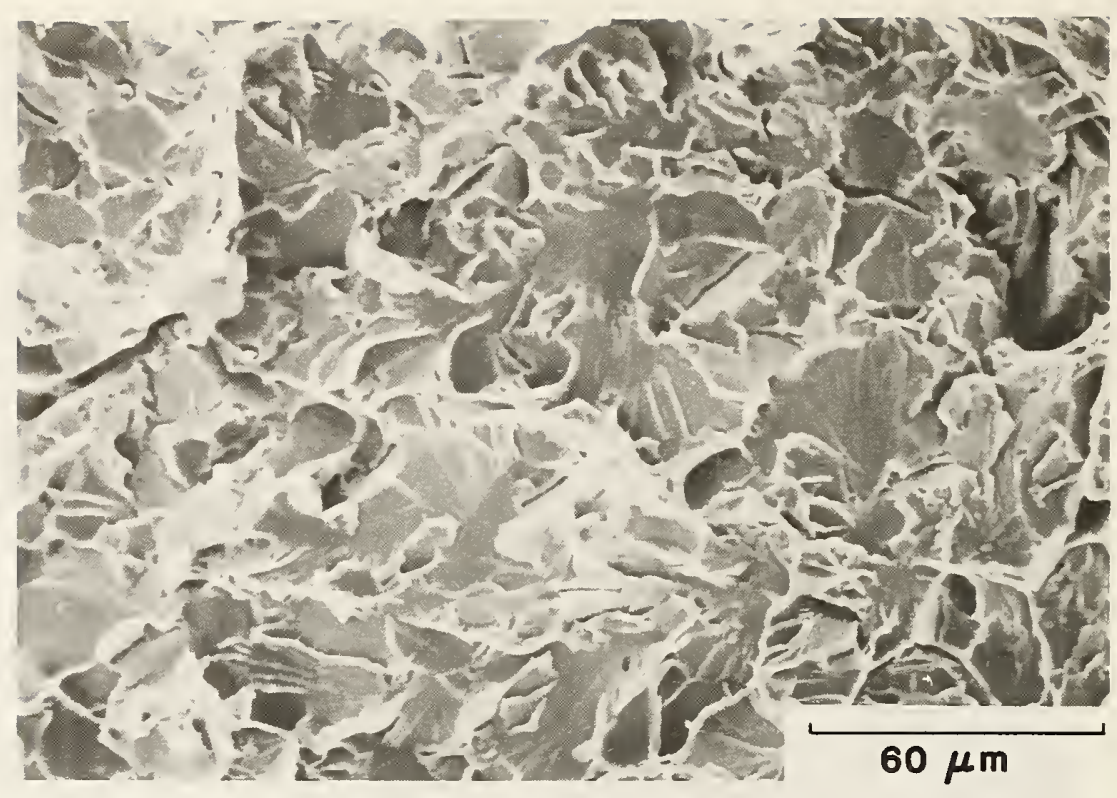

(a)

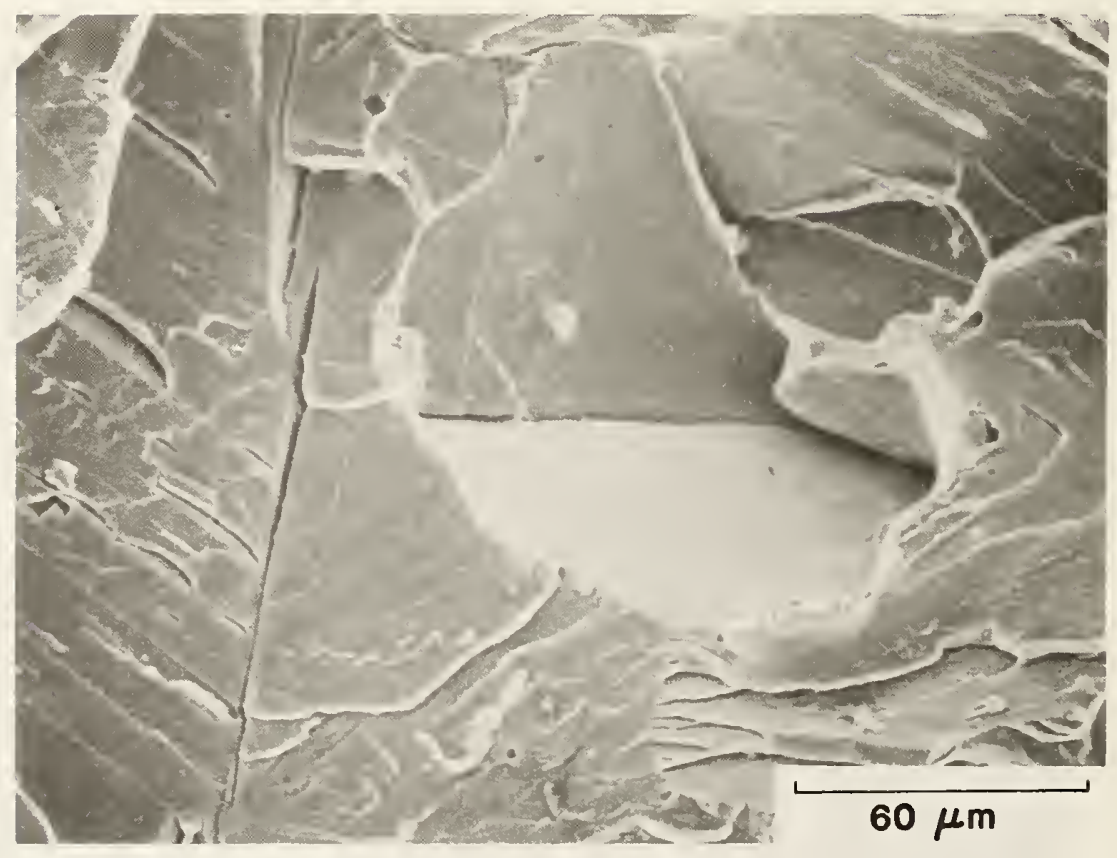

(b)

Figure 44. Comparison of fracture surfaces at $500 x$ for a) $A B S$ grade EH36 steel and b) commercially pure iron. 
transition region is mixed mode, the fraction of cleavage depending on how close a fracture is to the lower shelf. However, the fractographs show that the micromode of fracture can be pure cleavage in the transition region, even when a high degree of plastic flow has preceded fracture. The rapid increase in toughness at the transition occurs because cleavage becomes energetically unfavorable as temperature increases. The fracture is not mixed mode until the initiation toughness is exceeded. As illustrated in Figures 3 and 4 , the initation point for a clean steel can be well up the transition curve.

\subsubsection{Summary Comments}

Fracture toughness data for two materials as a function of specimen geometry and test temperature have been presented. The data exhibited the expected size effects in the ductile-to-brittle transition region. These effects were attributed to variations in crack-tip constraint. In Section 3.4, the concept of constraint is used to model the size effects on fracture toughness.

3.4 Characterization of Fracture in the Ductile-to-Brittle Transition Region

As stated in Chapter 1, the objective of this work was to characterize the size effects on fracture toughness and develop a constraint-based model by which the toughness of large structures can be estimated from smal1-scale fracture tests. 
Thus far, constraint has only been discussed qualitatively. For size effects to be expressed analytically in terms of constraint, an experimental measure of constraint is needed. The method by which these measurements were made is presented below.

Crack-tip region constraint (CTRC) is defined in this investigation as the degree of stress triaxiality near the crack tip; i.e.,

$$
\operatorname{CTRC}=\frac{\sigma_{y y}}{\bar{\sigma}}
$$

where $\sigma_{y y}$ is the stress normal to the crack plane (assumed to be equal to the maximum principal stress, $\sigma_{1}$ ) and $\bar{\sigma}$ is the effective stress.

The effective stress is defined by the von Mises equation:

$$
\begin{aligned}
& \bar{\sigma}=\frac{1}{\sqrt{ } 2}\left[\left(\sigma_{1}-\sigma_{2}\right)^{2}+\left(\sigma_{1}-\sigma_{3}\right)^{2}+\left(\sigma_{2}-\sigma_{3}\right)^{2}\right]^{\frac{1}{2}} \\
& \bar{\sigma} \leq \sigma_{0}
\end{aligned}
$$

where $\sigma_{0}$ is the yield stress. The effective stress is equivalent to the flow stress (at a given strain) in the absence of triaxiality. The analogous quantity, effective strain, is defined by equation (38):

$$
\bar{\varepsilon}=\frac{\sqrt{2}}{3}\left[\left(\varepsilon_{1}-\varepsilon_{2}\right)^{2}+\left(\varepsilon_{2}-\varepsilon_{3}\right)^{2}+\left(\varepsilon_{1}-\varepsilon_{3}\right)^{2}\right]^{\frac{1}{2}}
$$

where $\varepsilon_{1}, \varepsilon_{2}$ and $\varepsilon_{3}$ are principal strains. The $\bar{\sigma}$ versus $\bar{\varepsilon}$ flow curve is equivalent to the uniaxial true stress/true strain curve. Thus, a material's yielding and strain hardening behavior obtained from a 
uniaxial test can be related to triaxial conditions through equations (37) and (38) [81].

Equation (36) contains three unknowns. One unknown can be eliminated if $\bar{\varepsilon}$ at a given point ahead of the crack tip is known; $\bar{\sigma}$ can be inferred the flow curve. Section 3.4.1 describes how the author measured crack-tip region strain.

A model for cleavage fracture due to Ritchie, et. al. [38] was used to solve for $\sigma_{y y}$. According to this model, fracture occurs when the fracture stress, $\sigma_{f}$, is exceeded over a characteristic distance, $X_{c}$, ahead of the crack tip (see Fig. 7). Thus, at the moment of fracture, $\sigma_{y y}$ is equal to $\sigma_{f}$ at a distance $x_{c}$ ahead of the crack tip. The fracture stress is taken as the final load divided by the final area in a uniaxial tensile test in which the fracture mode is cleavage. The critical distance is inferred from Tracey's finite element crack-tip stress solutions [82] which are shown in Figure 45. The normal stress, $\sigma_{y y}$, on the ordinate is set equal to $\sigma_{f} ; K$ is set equal to a lower-shelf $K_{\text {Ic }}$ value; $\sigma_{0}$ is equal to the yield stress at that lower-shelf temperature. One can then solve for $X$ in Figure 45 and set it equal to $X_{C}$.

It was assumed that $\sigma_{f}$ and $X_{c}$ are material properties which are independent of temperature and constraint. Figure 46 is a plot of $\sigma_{f}$ versus temperature for the pure iron and the ABS EH36 steel obtained from low temperature tensile tests. The dashed 1 ines are drawn at one standard deviation above and below the mean. The fracture stress of the normalized steel is apparently insensitive to temperature. There 

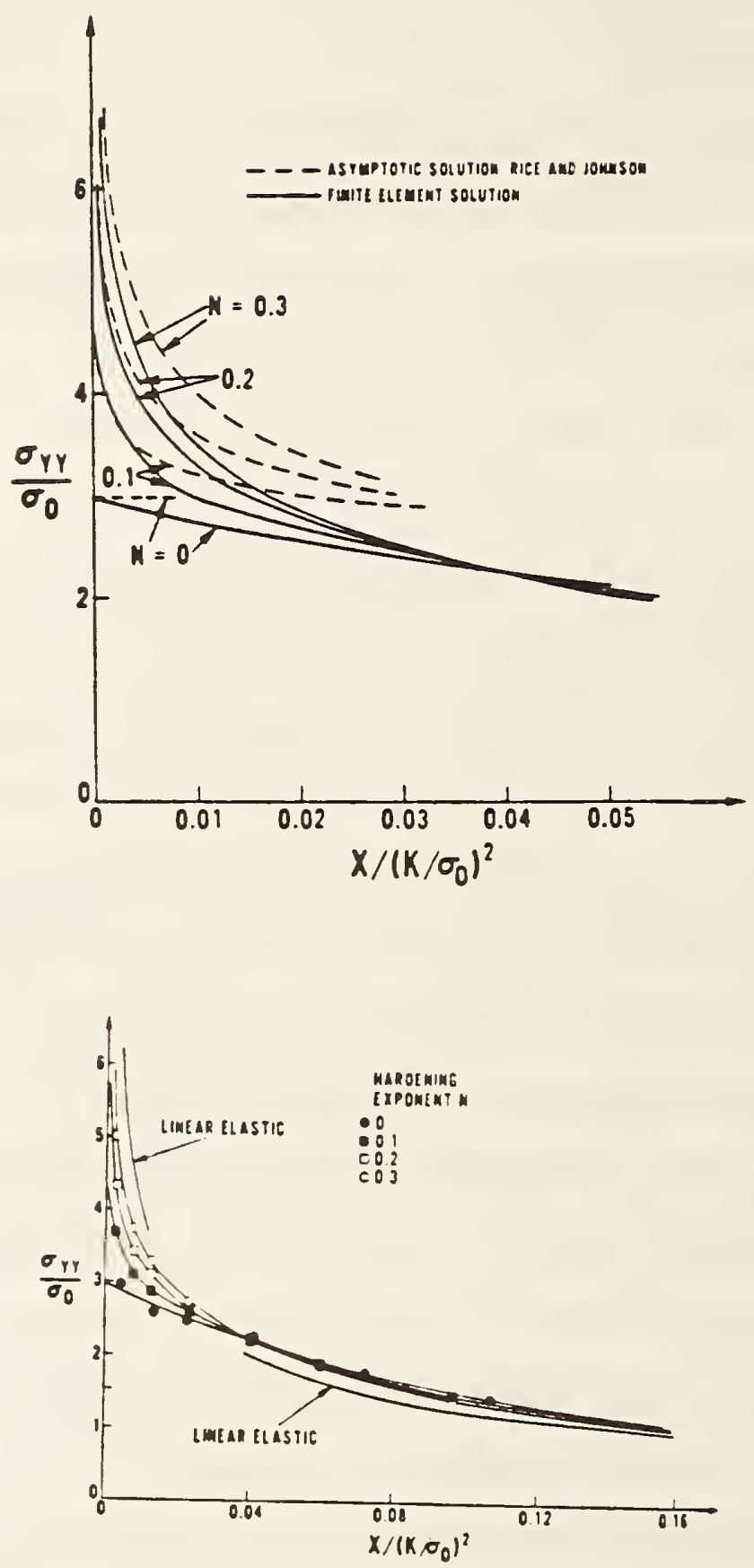

Figure 45. Finite element solutions for stress ahead of the crack tip (small-scale yielding); after Tracey [82]. 


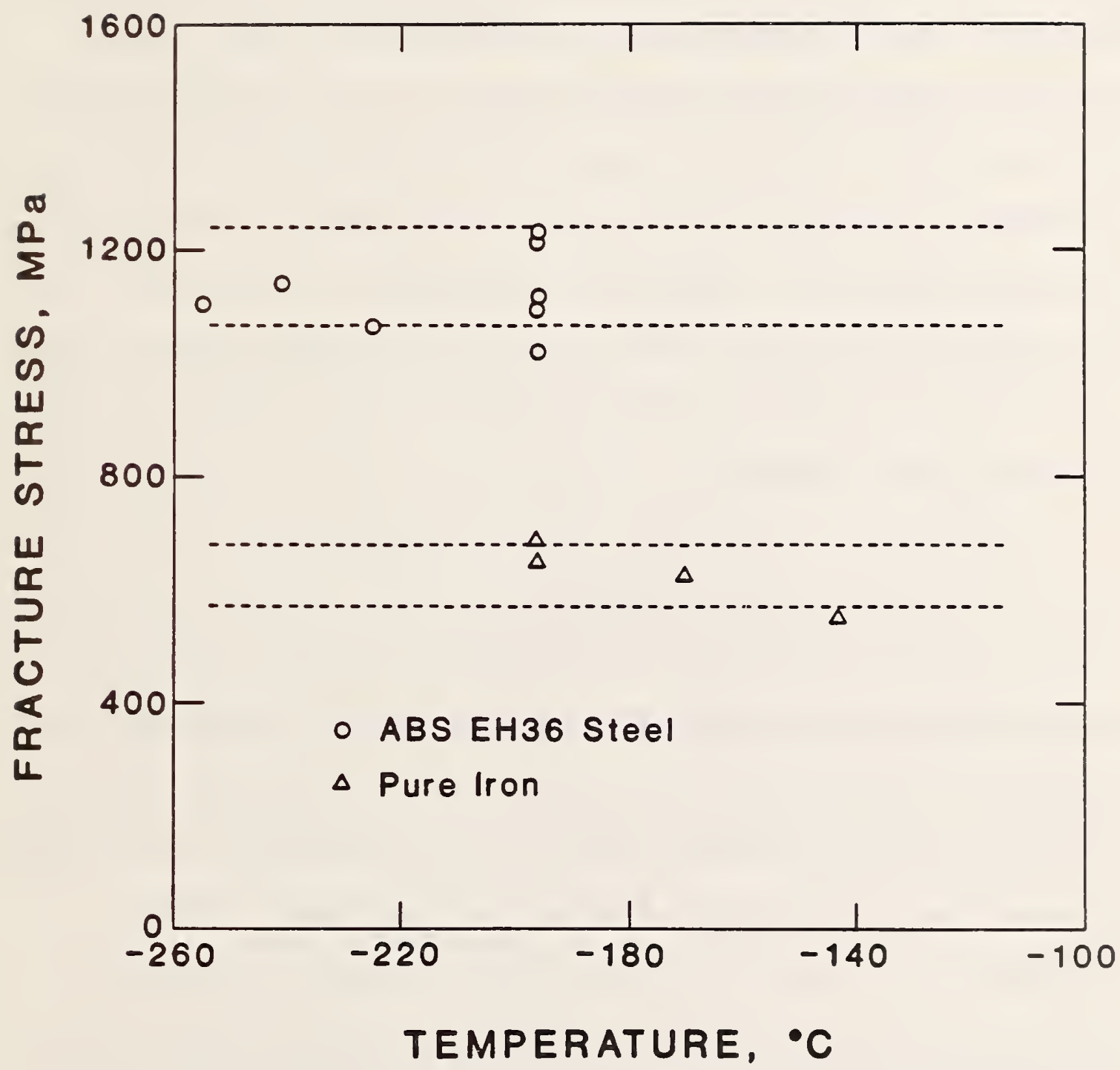

Figure 46. Cleavage fracture stress versus temperature for $A B S$ grade EH36 steel and pure iron tensile tests. 
appears to be a downward trend with increasing temperature in the pure iron data. However, since there are only four data points for this material it is difficult to tell whether or not this is a real trend. The assumption that $\sigma_{f}$ is independent of temperature was used for both materials. The mean values of $\sigma_{f}$ in Figure 46 are $1156 \mathrm{MPa}$ for the normalized steel and $627 \mathrm{MPa}$ for the pure iron. The assumption that $X_{c}$ is constant for a given material was partially justified in Section 1.3.1 where it was shown that, according to the model of Evans and Hutchinson [40], $X_{c}$ is independent of temperature. The Evans and Hutchinson model in only valid for small-scale yielding; however the data of Rosenfield, et. al., $[41,42]$ indicate that $X_{c}$ remains constant with large-scale yielding.

\subsubsection{The Crack-Tip Strain Function}

A knowledge of the strain field ahead of a blunted crack tip is necessary for constraint measurements. The effective flow stress at a given point ahead of the crack tip can be inferred from the flow curve if effective strain is known. Shoji et al. [83] measured strain ahead of crack tips in a steel with a bainitic microstructure. After deformation to a given CTOD, the specimen was annealed to nucleate ferrite grains in the intense strain region at the crack tip. The recrystallized ferrite grain size was calibrated with prior plastic strain. Shoji obtained the following empirical relationship for strain as a function of $\delta$ and distance from the crack tip. 


$$
\bar{\varepsilon}=0.25\left(\frac{\delta \cos \theta}{r}\right)^{0.65}
$$

where $r$ is the distance from the crack-tip free surface and $\theta$ is the angle from the crack plane to the point in question.

Other Investigators $[84,85]$ have used microhardness correlations to determine the crack tip strain distribution. This method was used here because microhardness seems to be a more direct and sensitive measure of strain than recrystalized grain size. Microhardness tests were used in this investigation to measure crack-tip strain. Initial attempts to measure strain with crack-tip microhardness tests on the ABS EH36 steel were unsuccessful. The fine, two-phase microstructure of this steel made reproducible hardness measurements impossible. The pure iron is much better suited to this technique because it is an essentially single-phase material with very large grains.

The microhardness/strain calibration method is outlined in Figure 47. The diameters of two fractured tensile bars were measured (from enlarged photographs) at various locations in the necked-down region. The reduction in diameter was used to compute plastic strain:

$$
\varepsilon_{p}=2 \ln \left(\frac{D_{0}}{D}\right)
$$

where $D_{0}$ is the original diameter of the tensile specimen. The plastic strain was plotted against distance from the fracture surface. This plot is shown in Figure 48 for two pure iron tensile specimens. Each 

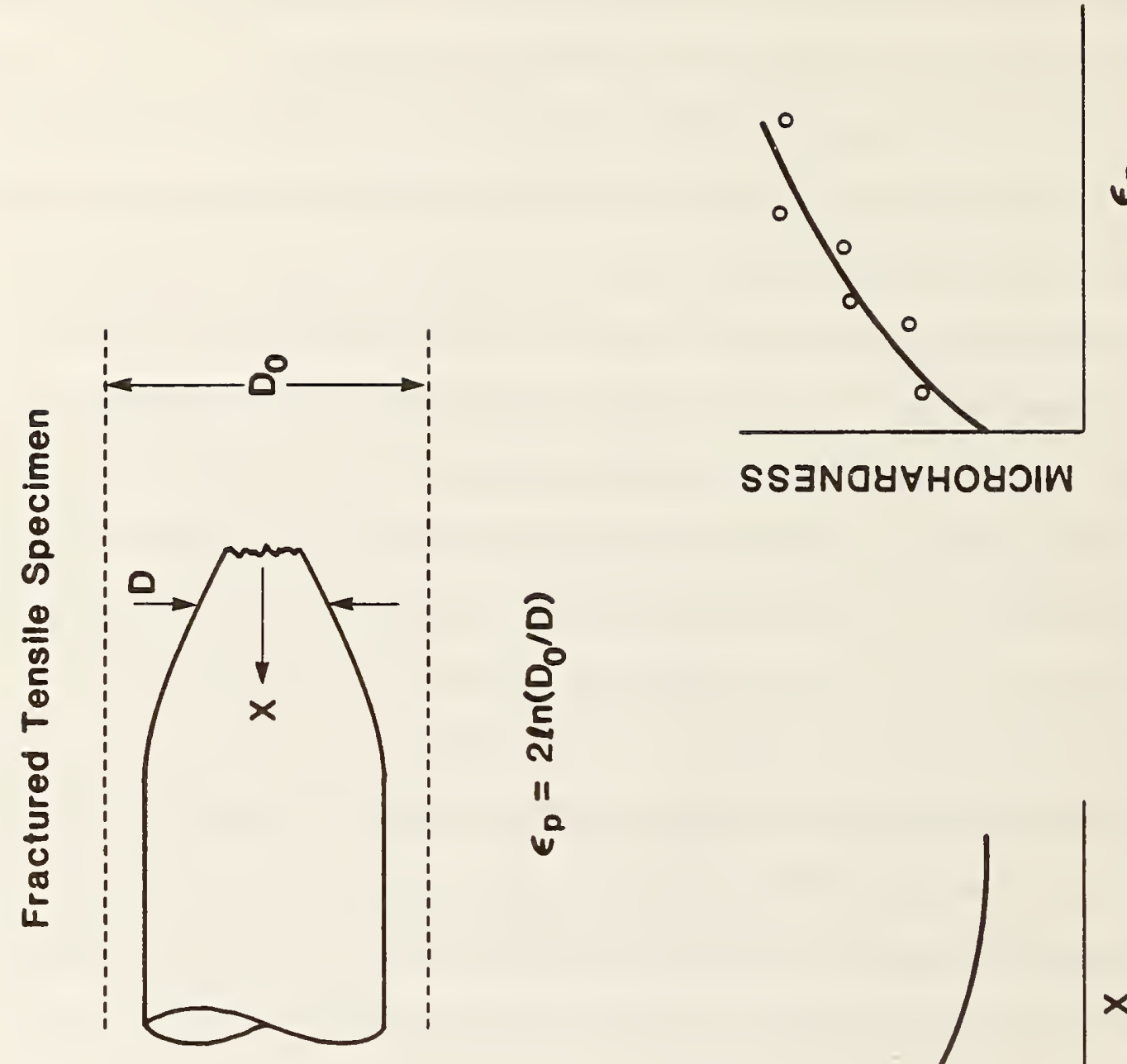

"

$\because \frac{1}{4}$

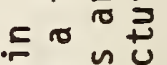

옹동늉

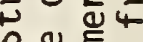

Uิ

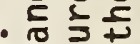

ct n

응 응

0
0
$\frac{2}{2}$
N
11
w

\%

ᄂ. nै.

$\approx$ \&ै

훙든도

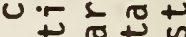

든응

은 인단

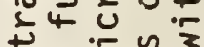

औ

nิ n 음

น

등

ช。ำ

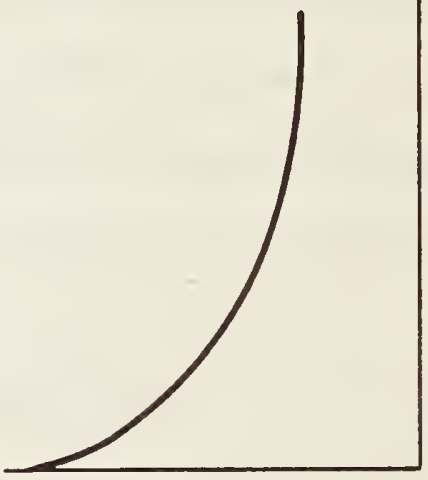

w

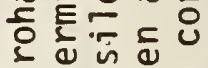

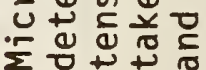

\begin{tabular}{l}
\multirow{8}{*}{} \\
$\frac{0}{3}$ \\
$\frac{1}{4}$
\end{tabular} 


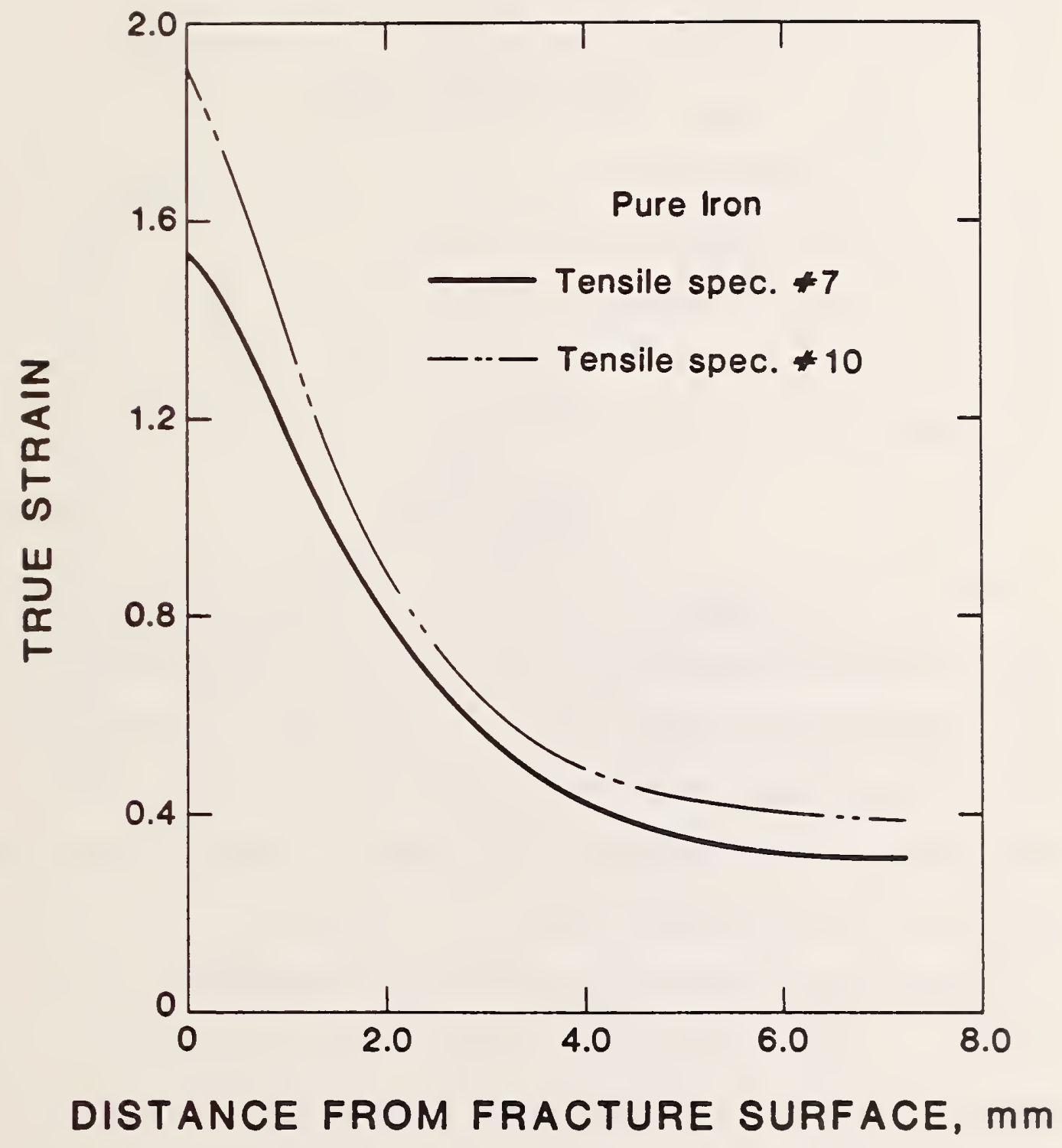

Figure 48. Plastic strain as a function of distance from the fracture surface in two pure iron tensile specimens. 
specimen was then sectioned and microhardness measurements were taken at various distances from the fracture surface. The diamond pyramid hardness test with a load of $50 \mathrm{~g}$ was used for all microhardness measurements. Distance was converted to strain from Figure 48 and microhardness values were plotted against the corresponding strain values. Figure 49 shows a plot of microhardness versus $\varepsilon^{2 / 3}$, which is a straight line. Each data point in Figure 49 represents the average of 8-10 microhardness measurements at a constant strain. In addition to the two necked-down specimens, unstrained material and two specimens (\#1 and \#2) uniformly deformed to different strains were used for the microhardness/strain calibration. According to Figure 49:

$$
\bar{\varepsilon}=\left(\frac{\mathrm{DPH}-105}{80}\right)^{1.5}
$$

Two pure iron SENB specimens were used to determine the crack-tip strain function. Each specimen was deformed at room temperature to a given CTOD and then unloaded. Next, the specimens were sectioned in the center, perpendicular to the crack plane (see Fig. 39b). After the specimens were mounted, polished and etched, microhardness measurements were taken at various points ahead of the crack tip, on the crack plane. The microhardness indentations were spaced at approximately $100 \mu \mathrm{m}$ intervals. These indentations were then removed by grinding so that additional microhardness measurements could be made. This process was repeated several times in order to provide enough data to compute reliable mean values. Figure 50 shows a logarithmic plot of strain 


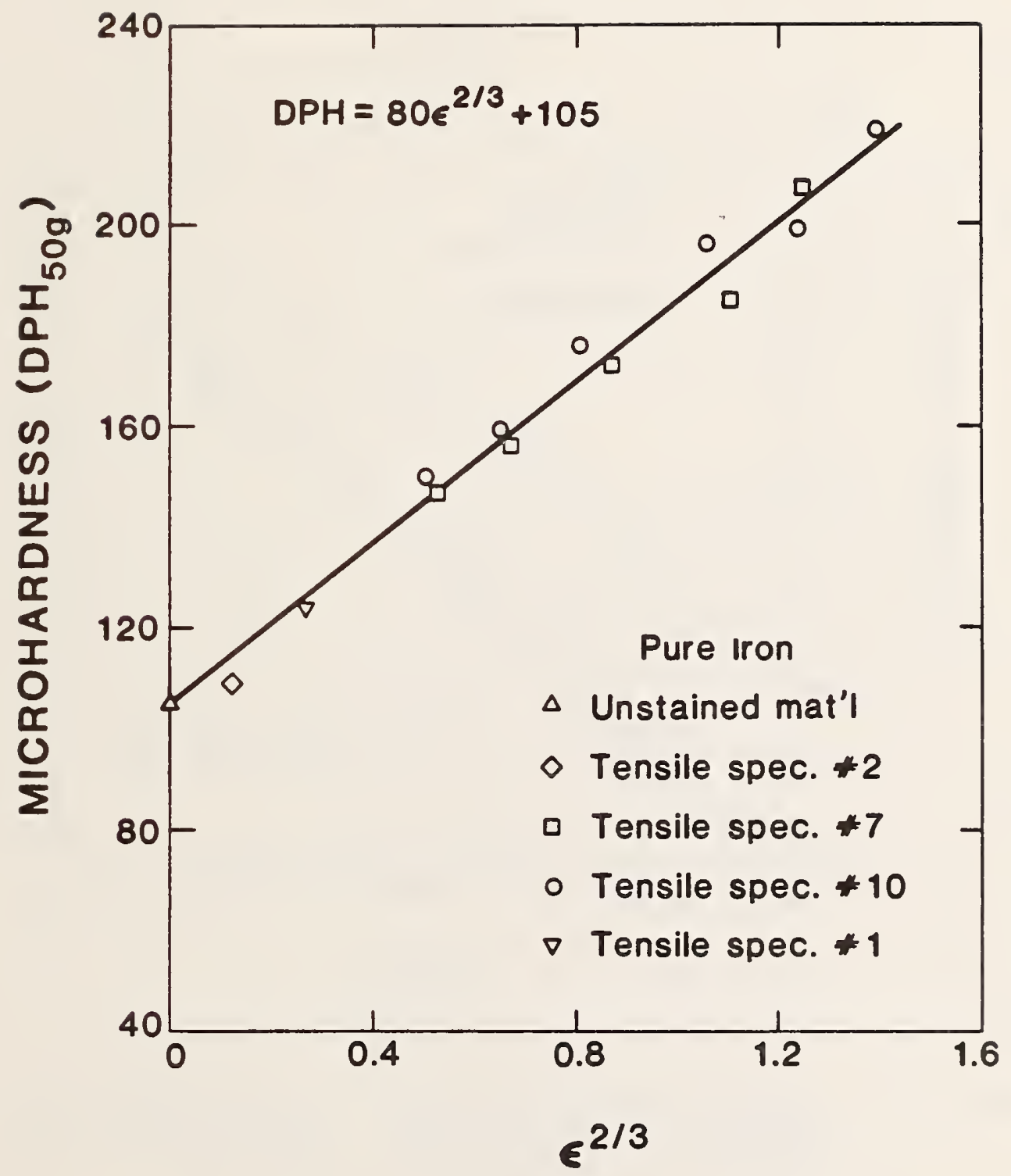

Figure 49. Diamond pyramid hardness (load $=50 \mathrm{~g}$ ) as a function of plastic strain in pure iron. 


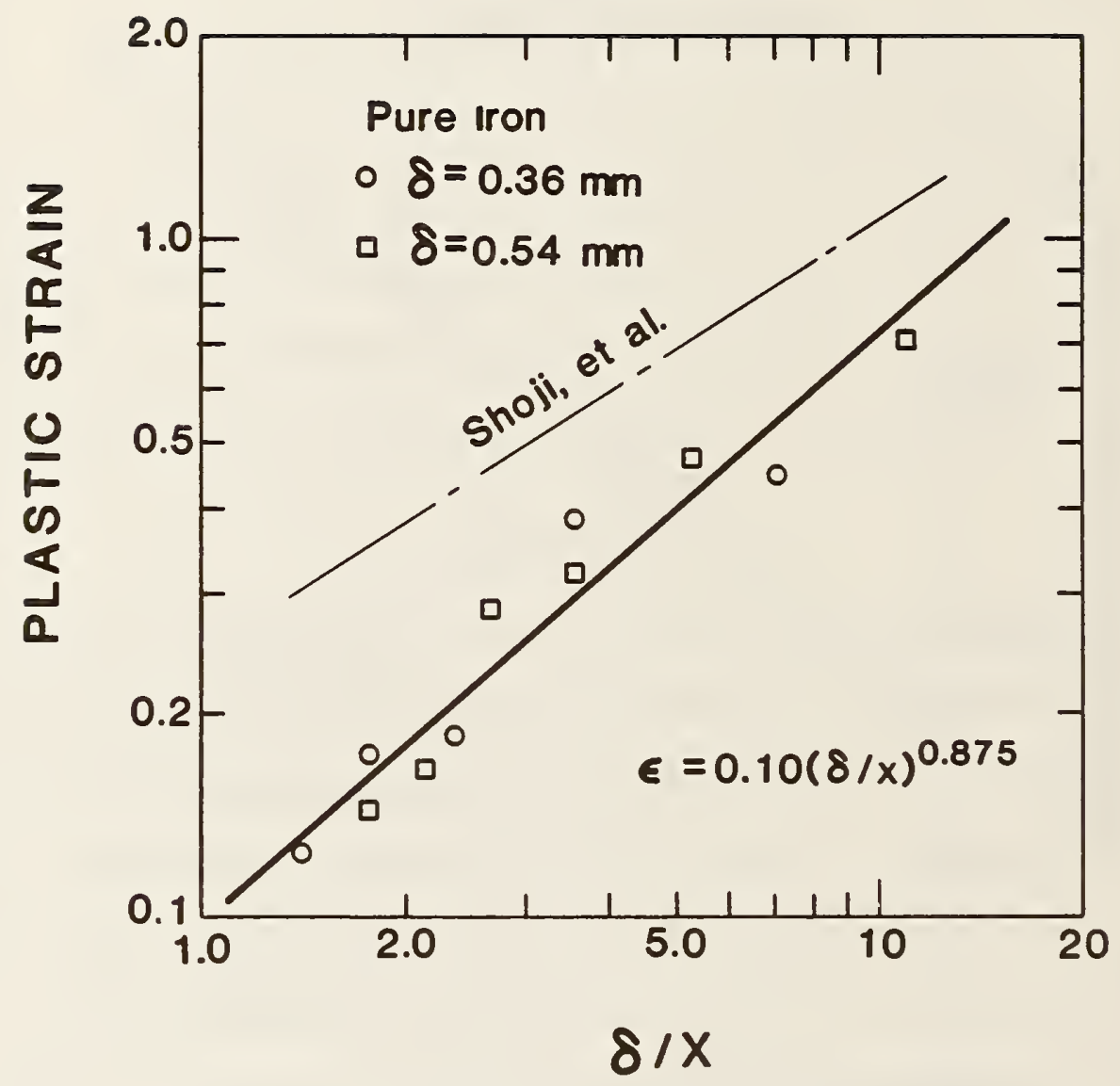

Figure 50. Crack-tip region strain as a function of CTOD and distance from the crack tip. The Shoji [61] striin function is superimposed for comparison. 
versus $\delta / X$, where $X$ is the distance from the crack tip. Each data point in Figure 50 represents the average of 4-6 microhardness measurements. The strain function from Figure 50 is given below.

$$
\bar{\varepsilon}=0.10\left(\frac{\delta}{x}\right)^{0.875}
$$

The Shoji [83] strain equation is represented by the dashed line in Figure 50. There is a significant difference between equations (39) and (42). However, equation (38) was obtained for a steel with a bainitic microstructure. The materials of interest here have ferritic or ferrite/pearlite microstructures.

Although equation (42) was defined for the pure iron, it was assumed that this equation also describes the strain field ahead of a crack in ABS grade EH36 steel. The justification for this assumption is as follows: Cracks in both materials blunt in approximately a semicircle (see Fig. 39); i.e., blunting cracks in both materials correspond to the same displacement field. For a given displacement field, the correpsonding strain field should not be a strong function of material. Also, the flow characteristics of both materials should be similar since the primary microstructural constituent in each material is ferrite, which should dominate the flow properties. 


\subsubsection{Measurement of CTRC}

With the strain function (eq. 42) and experimental values for fracture stress and critical distance, there is now enough information to solve for constraint in equation (36). The crack-tip region constraint was measured for three geometries of normalized steel SENB specimens: 1) $B=0.5 W=12.7 \mathrm{~mm}, a / W \sim 0.2$; 2) $B=W=25.4 \mathrm{~mm}, a / W \sim 0.2$; 3) $B=W=25.4 \mathrm{~mm}, a / W \sim 0.5$. The average value of $\sigma_{f}$ for this material is $1156 \mathrm{MPa}$ (see Fig. 46); a value of $0.215 \mathrm{~mm}$ was computed for $X_{c}$ from Figure 45. It was assumed that these parameters remain constant with temperature and geometry variations. The procedure which was used to measure CTRC is outlined below.

1) A critical CTOD value in the ductile-to-brittle transition region was taken from Table 7 .

2) For a given $\delta_{C}$ value, $\bar{\varepsilon}$ at $X_{C}$ was computed:

$$
\bar{\varepsilon}_{c}=0.10\left(\frac{\delta_{c}}{x_{c}}\right)^{0.875}
$$

where $\bar{\varepsilon}_{c}$ is the strain at $X_{c}$ at the moment of fracture; $X_{c}=$ constant $=0.215 \mathrm{~mm}$.

3) The effective stress corresponding to a strain of $\bar{\varepsilon}_{c}$ is inferred form the uniaxial true stress/true strain curve at the temperature of the corresponding $\delta_{c}$ value. Figure 51 shows that the strainhardening rate of the normalized steel is insensitive to 


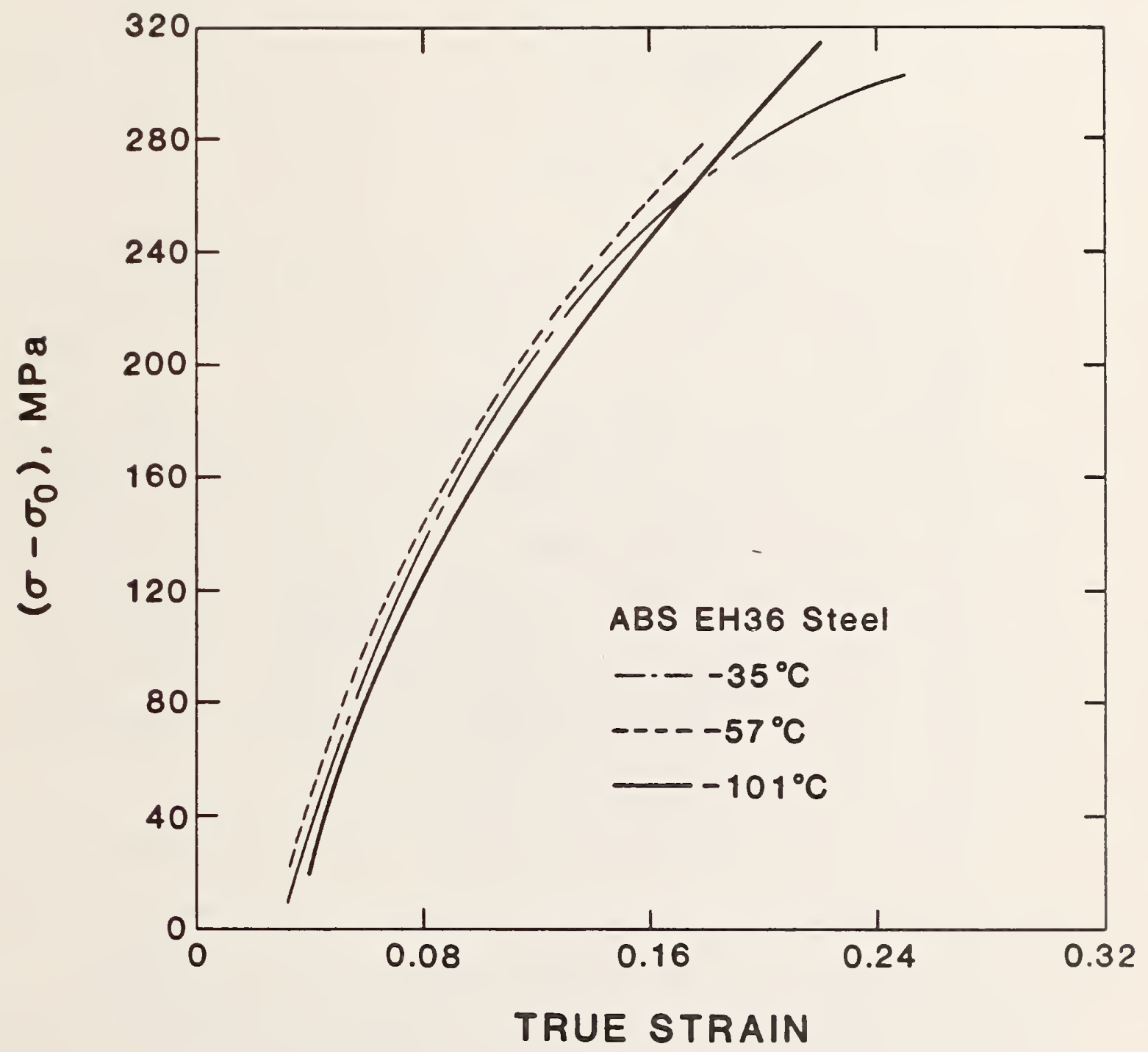

Figure 51. True stress/true strain curves normalized for temperature for ABS grade EH36 steel. 
temperature in the region of interest. The average of the three curves in Figure 51 represents a unified flow curve which is valid over a wide range of temperatures.

4) CTRC was computed from equation (44):

$$
\text { CTRC }=\frac{\sigma_{f}}{\bar{\sigma}_{C}}
$$

where $\sigma_{f}=$ constant $=1156 \mathrm{MPa}$.

5) Steps 1 through 4 were repeated for various temperatures and geometries .

Critical crack-tip region constraint is plotted as a function of critical CTOD in Figure 52. Constraint decreases markedly with CTOD. Apparently crack-tip blunting causes a relaxation in triaxiality. At a constant CTOD the constraint tends to be lowest for the thinnest specimens $(B=0.5 \mathrm{~W}=12.7 \mathrm{~mm})$. This was expected since this geometry exhibits a ductile-to-brittle transition in the lowest temperature range (see Figs. 23 and 24 ).

It should be noted that critical CTOD values above approximately $0.5 \mathrm{~mm}$ are $\delta_{u}$ values; i.e., some stable tearing has preceded fracture. The above analysis is not strictly valid if tearing has occured. However, critical CTOD values up to $0.8 \mathrm{~mm}$ are plotted Figure 41 because the amount of tearing is small. This small degree of tearing apparently 


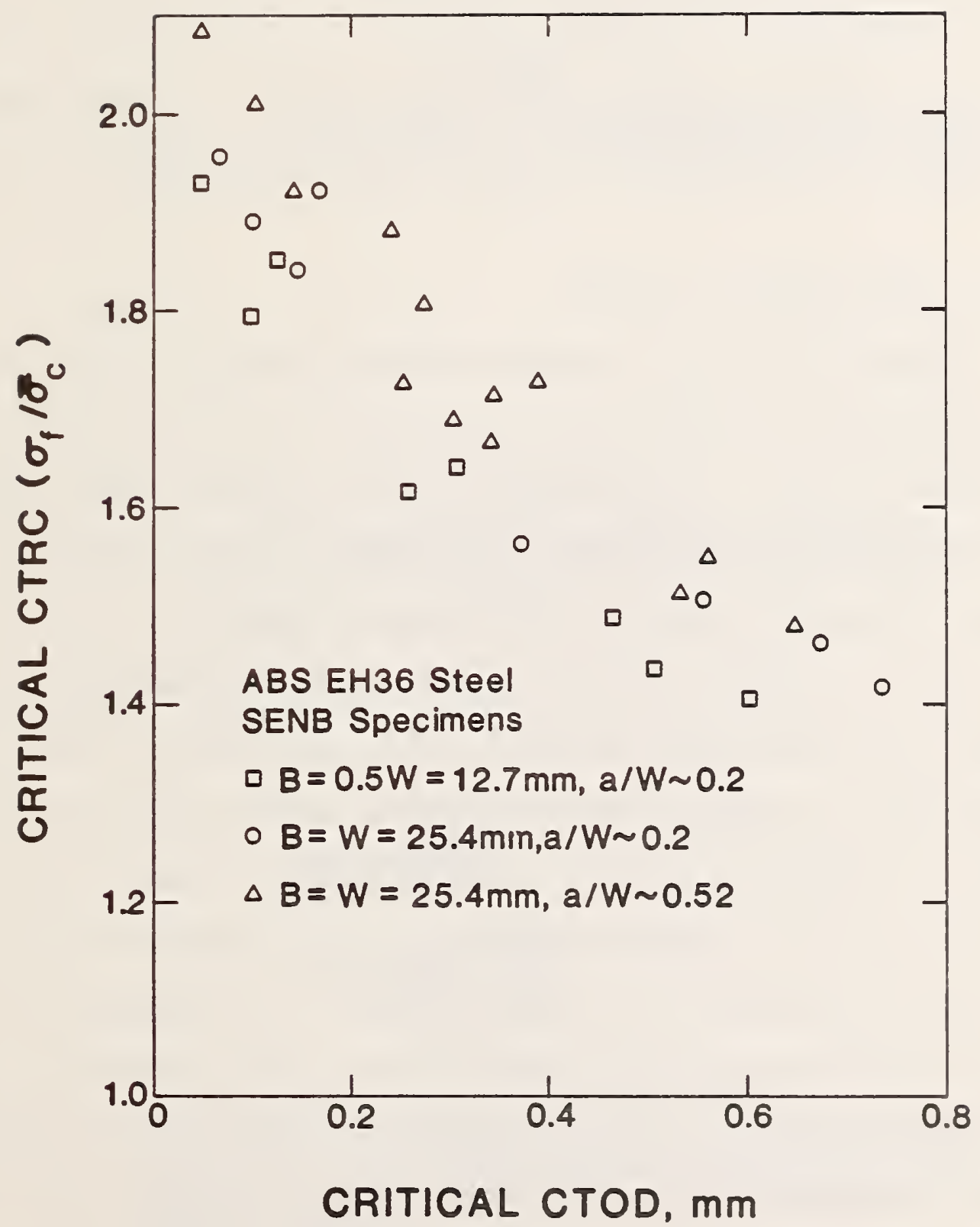

Figure 52. Critical crack-tip region constraint as a function of critical CTOD for three SENB specimen geometries of ABS grade EH35 steel. 
has little effect since the high toughness data in Figure 52 do not deviate from the trend established by the other data.

No curves are drawn through the data in Figure 52 because, up to this point, no functional relationship between CTRC and critical CTOD has been established. The determination of a functional relationship requires a model for the observed material behavior. One such model for the constraint relaxation at a crack tip is proposed below.

\subsubsection{A Spring Model for CTRC}

Although the physical situation described above is complex (i.e., a three-dimensional elastic-plastic continuum) the model presented here is relatively simple. This approach is justified because complex models of natural phenomena are often preceded by more rudimentary versions. These simple analyses often provide valuable insights.

The relaxation in constraint with crack blunting was modeled by means of an elastic spring analog. This is illustrated in Figures 53 and 54 where an element of material which is loaded to the fracture stress is constrained laterally by linear elastic springs in tension. The spring in the $x$ direction is assumed to be connected at the crack tip and the hinge point (in a bend specimen). It is assumed that the crack is sharp initially; the initial stress in the $x$ direction is $\sigma_{x}^{0}$. The crack is assumed to blunt in a semicircle, resulting in a relaxation in the $x$ direction of the spring of $\delta / 2$. The force relaxation is given by 
crack Tip $\underset{x_{c}}{\operatorname{rom}} \prod_{\downarrow}^{1 \sigma_{t}} \operatorname{rowhorm} \rightarrow \sigma_{x}^{0}$

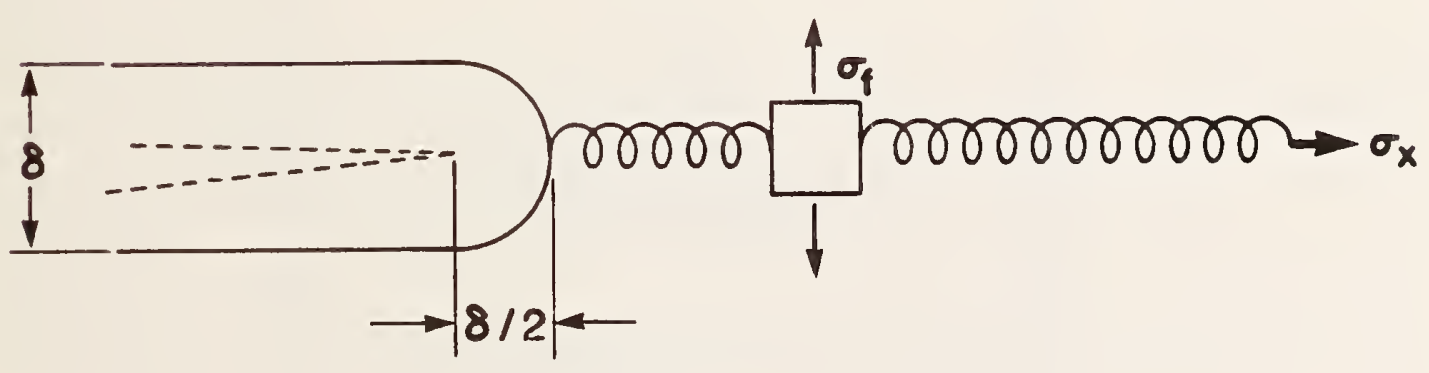

$$
\begin{aligned}
\Delta F_{x} & =\frac{-k \delta}{2} \\
\sigma_{x} & =\sigma_{x}^{0}-\frac{k \delta}{2 A}
\end{aligned}
$$

Figure 53. Spring model for CTRC relaxation. The crack tip blunts in a semi circle, thereby relaxing the spring which is causing a lateral tensile stress in the material element. 


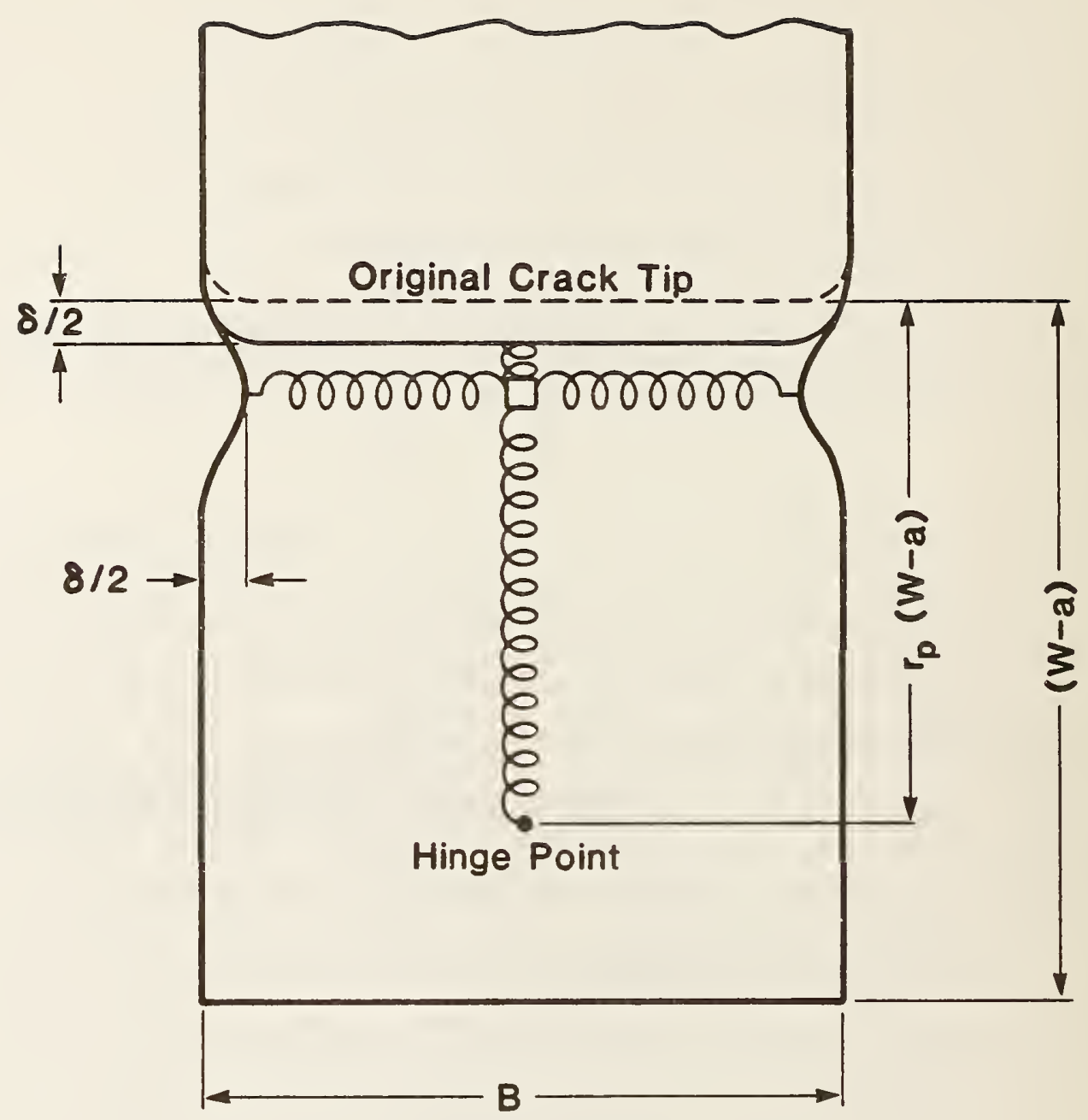

Figure 54. Constraint relaxation in the thickness and ligament directions. 


$$
\Delta F_{x}=\frac{-k \delta}{2}
$$

where $k$ is the spring stiffness.

Thus, the stress in the $x$ direction is given by

$$
\sigma_{x}=\sigma_{x}^{0}-\frac{k \delta}{2 A}
$$

where $A$ is the area over which the spring acts. There is also a relaxation in the $z$ direction due to lateral constraint (Fig. 54). The Tresca yield criterion can be used to solve for $\bar{\sigma}$ :

$$
\bar{\sigma}=\sigma_{\max }-\sigma_{\min }
$$

where $\sigma_{\max }$ is the maximum principle stress and $\sigma_{\min }$ is the minimum principle stress. If it is assumed that $\sigma_{x}<\sigma_{z}<\sigma_{f}$, then

$$
\bar{\sigma}_{c}=\sigma_{f}-\sigma_{x}^{0}+\frac{k \delta_{c}}{2 A}
$$

The Tresca criterion was used instead of the von Mises criterion (eq. 37) in order to simplify equations.

Equation (48) states that the critical effective stress is 1 inearly dependent on CTOD. These two quantities are plotted in Figure 55; best-fit straight lines were computed by linear regression analysis. The experimental values of critical effective stress in Figure 55 were obtained by means of the first three steps outlined in Section 3.4.3. 


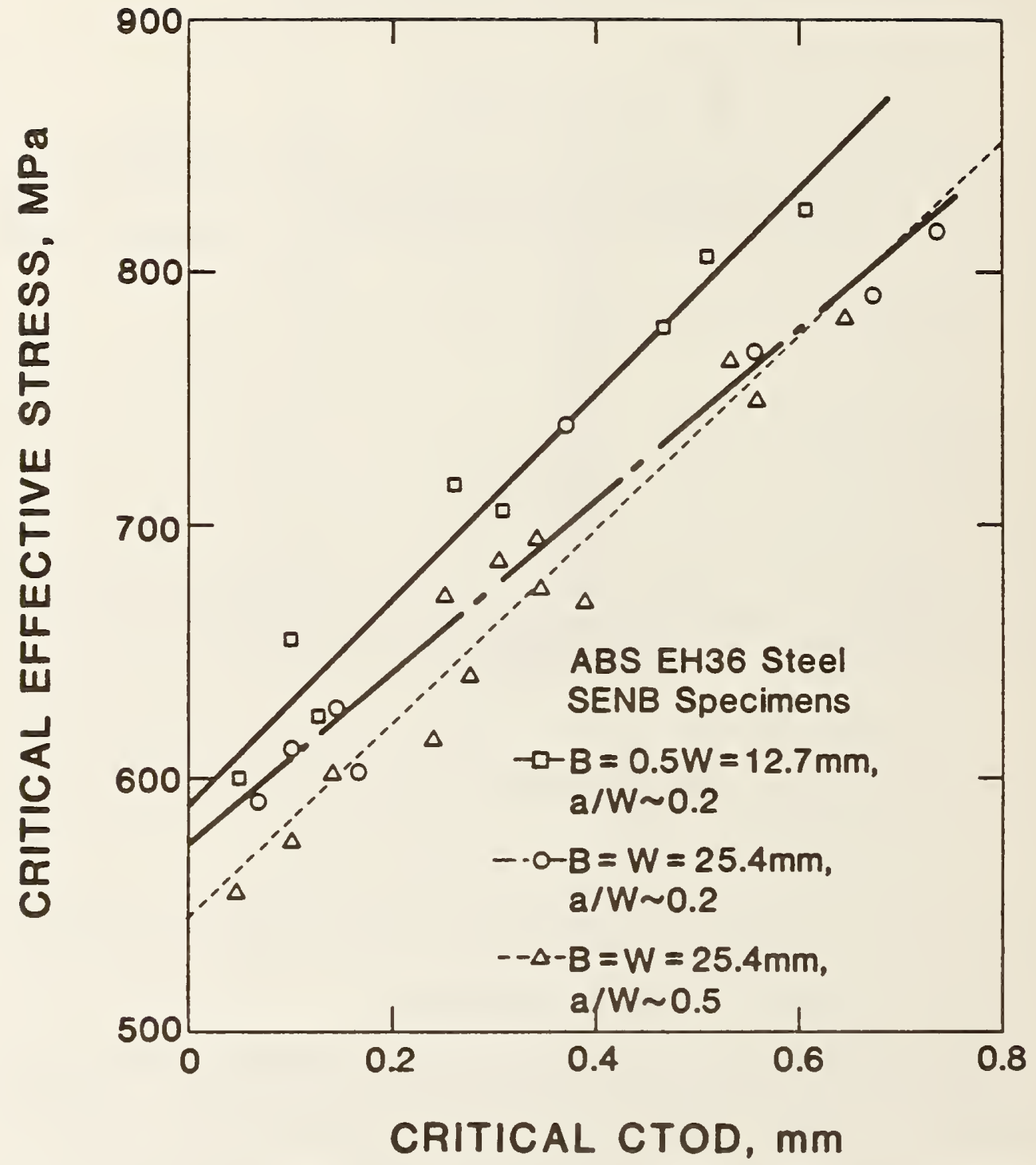

Figure 55. Critical effective stress as a function of critical CTOD for three SENB geometries of ABS grade EH36 steel. 
These are essentially the same data as were plotted in Figure 52, except that the fracture stress (which is a constant) was divided by $\bar{\sigma}_{C}$ to obtain the critical CTRC values in Figure 52. Despite the simplicity of the model, equation (48) correlates well with experimental data. The coefficients of determination for the three geometries plotted in Figure 55 range from 0.939 to 0.975 .

Each specimen geometry in Figure 55 exhibits a different slope and intercept. According to equation (48) the intercept is at $\sigma_{f}-\sigma_{x}^{0}$. Since the fracture stress is a material constant the initial laterial stress, $\sigma_{x}^{0}$, must be geometry dependent. The slope of each of the curves in Figure 55, which will hereafter be called the relaxation slope, is equal to $k / 2 A$, where $k$ is the spring stiffness.

The relaxation slope and intercept for various specimen configurations of ABS grade EH36 steel are listed in Table 10. (Data from Tables $7 \mathrm{f}$ and $7 \mathrm{~g}$ are not included because the large scatter and small sample population made it impossible to establish meaningful trends.)

The relaxation slope tends to decrease with increasing ligament length in the bend specimens. This can be explained qualitatively in terms of the inverse relationship between the length and stiffness of a spring; i.e., a short spring is stiffer than a long spring. The length of a spring is related to the ligament length. The relationship between relaxation slope and ligament length is quantified later. 
The constraint relaxation behavior for the three tension geometries is different than that of SENB specimens. Table 10 shows that the ligament length of an SENT specimen has little effect on the relaxation slope. However, when the thickness is increased by a factor of three the relaxation slope decreases significantly. Apparently the contraction in the thickness direction governs the constraint relaxation, even if the ligament length is much less than the thickness.

\subsubsection{A Model to Estimate $\sigma_{x}^{0}$}

As stated above, the initial lateral stress is geometry dependent. The spring model has been extended to derive an expression for this stress. For the purpose of this derivation, the initial lateral stress which governs constraint was assumed to act in the $x$ direction.

Consider the case where fracture in an SENB specimen coincides with the onset of net-section yielding. The amount of plastic flow prior to fracture would be small; the crack would be relatively sharp. The following assumptions can therefore be made:

$$
\begin{aligned}
& \sigma_{x}=\sigma_{x}^{0} \\
& \bar{\sigma}_{c}=\sigma_{0}
\end{aligned}
$$

Also, since the ligament is on the verge of yielding, the fracture load, $P_{f}$, is equal to the yield load. Thus, according to equation (35) 


$$
P_{f}=\frac{L \sigma_{0}(W-a)^{2} B}{4 W}
$$

Solving for $\sigma_{0}$ leads to

$$
\sigma_{0}=\bar{\sigma}_{c}=\frac{4 P_{f} W}{(W-a)^{2} B L}=\sigma_{f}-\sigma_{x}^{0}
$$

One can then solve for $\sigma_{x}^{0}$ :

$$
\sigma_{x}^{0}=\sigma_{f}-\frac{\sigma_{\text {nom }}}{L}
$$

where $\sigma_{\text {nom }}=\frac{4 P_{f} W}{(W-a)^{2} B}=$ nominal 1 igament stress at fracture.

The nominal stress at fracture for the normalized steel is approximately $770 \mathrm{MPa}$. This quantity seems to be insensitive to geometry. Thus,

$$
\frac{\sigma_{x}^{0}}{\sigma_{f}}=1-\frac{0.67}{L}
$$

Equation (52) is plotted in Figure 56 along with experimental values for initial lateral stress as a function of notch constraint. The predicted curve lies above the data but the trends of the data and the curve are similar. A vertical line is drawn on Figure 56 at $L=\sqrt{3}$. This represents the upper limit of elastic constraint. The value of $\sqrt{3}$ is an approximate upper bound of $L$ al though $L$ can exceed $\sqrt{3}$ slightly because the specimen behavior is elastic-plastic.

The fact that $\sigma_{x}^{0}$ and $L$ may be simply related is encouraging because it means prediction of transition curves as a function of geometry is possible. While multiple specimens at various temperatures are required 


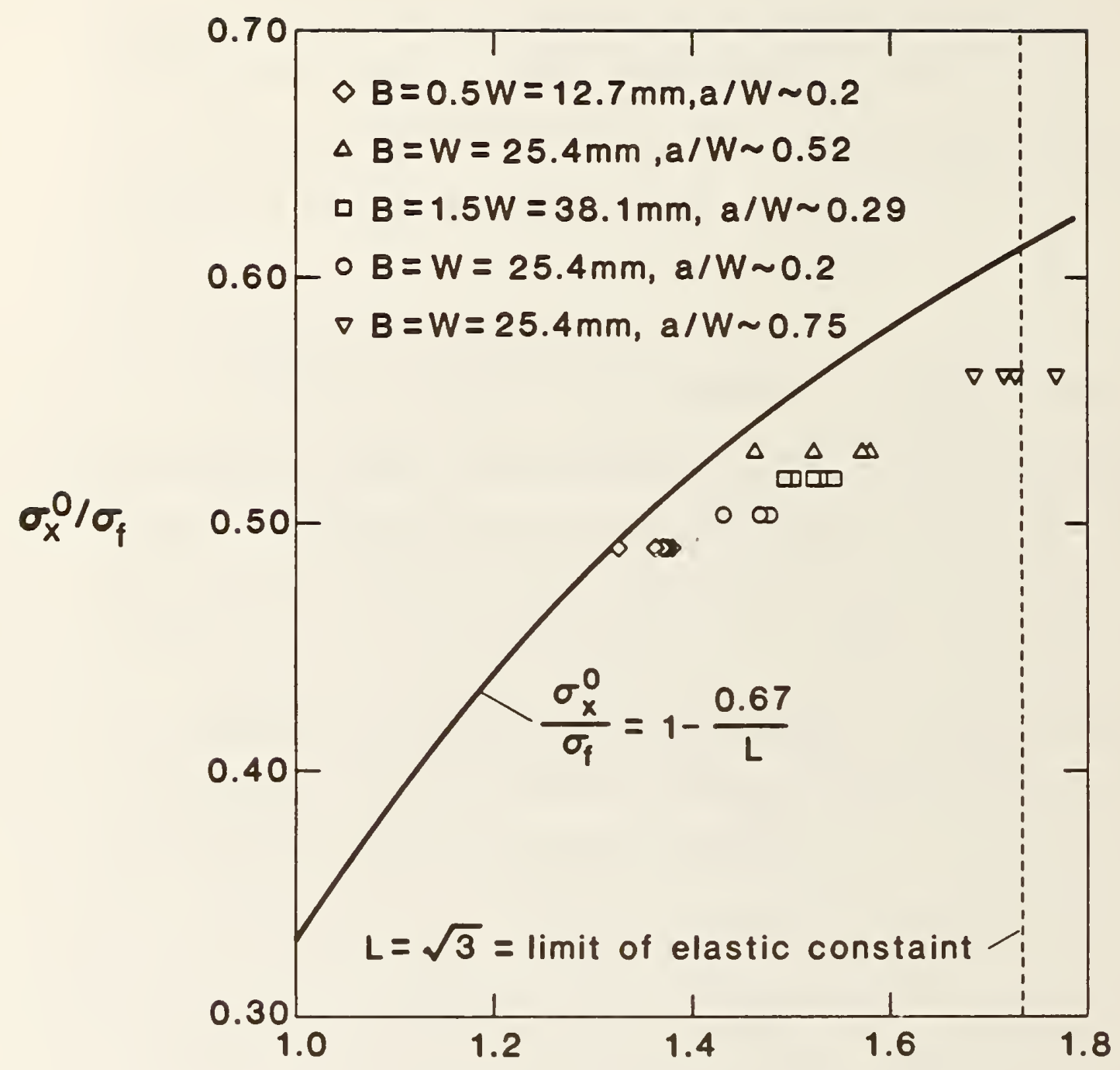

NOTCH CONSTRAINT FACTOR (L)

Figure 56. Comparison of predicted and experimental initial lateral stress as a function of notch constraint for ABS EH36 steel SENB specimens. 
to determine $\sigma_{x}^{0}$ for a given geometry (Fig. 55), L can be determined from a single test at any temperature.

\subsubsection{Predicting the Relaxation Slope}

According to Table 10 , the slope of the relaxation curve, $k / 2 A$, is dependent on ligament length for bend specimens and apparently on thickness for tensile loading. In the analyses that follow, only the case of the SENB geometry, where constraint relaxation is primarily in the $x$ direction, is considered.

In order to obtain estimates of the relaxation slope, it was necessary at this point to replace the linear elastic spring with a bar of length $r_{p} b$, where $b$ is the ligament length, $w-a$, and $r_{p}$ is the rotational factor (eq. 31 ). This bar is illustrated at the top of Figure 57. If the bar is displaced a distance $\delta / 2$, a strain field will be introduced into the bar. A uniform strain field will be introduced if the displacement field is linear along the length of the bar (Fig. 57, center). A displacement field can be applied such that the strain

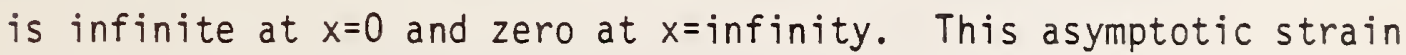
field, which is illustrated at the bottom of Figure 57 , should be representative of the real $\varepsilon_{x}$ field ahead of a blunted crack tip.

The strain field in the bar should have the form:

$$
\varepsilon_{x}=A\left(\frac{\delta}{x}\right)^{0.875}
$$



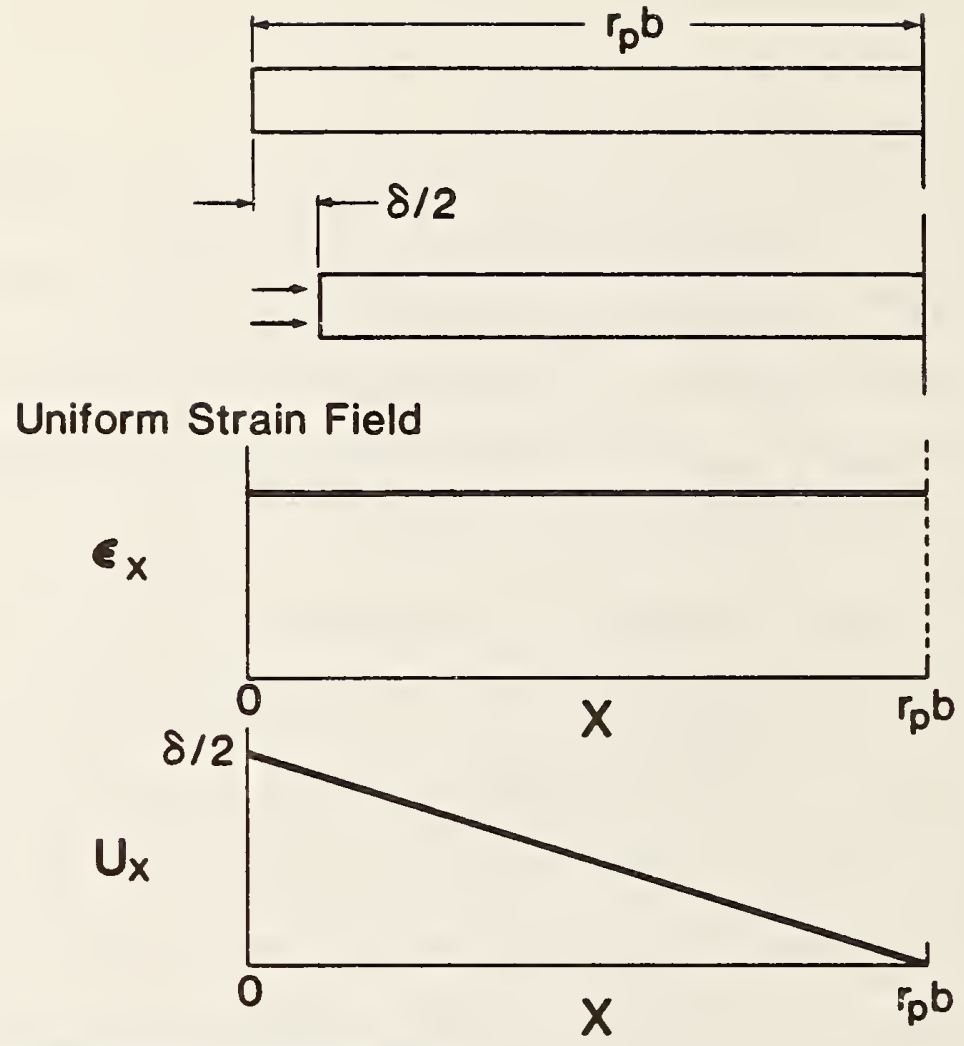

Decaying Strain Field

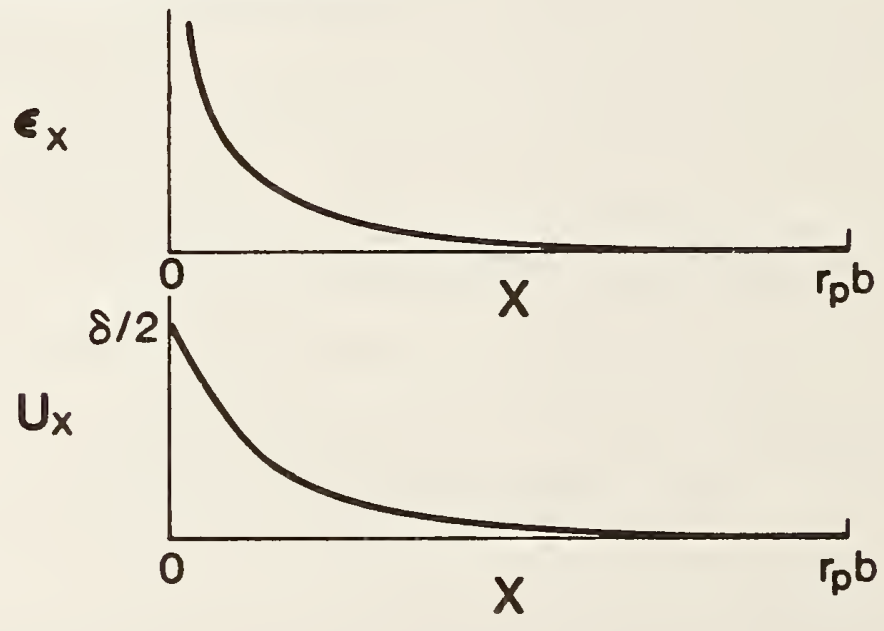

Figure 57. Schematic of a bar displaced by $\delta / 2$ with corresponding uniform and asymptotic strain fields. 
The exponent in equation (53) must be the same as that in equation (42) because $\varepsilon_{x}$ is simply related to the other principle strains and $\bar{\varepsilon}$ through incompressibility equations. In order to satisfy these equations at all values of $x$ and $\delta$, the exponents on each of the principle strains and $\bar{\varepsilon}$ must be the same.

If the small strain approximation (which is reasonably accurate for strains up to 0.10 ) is used, equation (53) can be integrated with respect to $x$ to determine the displacement field:

$$
u_{x}=\frac{A \delta^{0.875} x^{0.125}}{0.125}+C
$$

One can solve for the constants $A$ and $C$ by applying the following boundary conditions (see Fig. 57, bottom).

$$
\begin{array}{ll}
\text { at } x=0 & u_{x}=\delta / 2 \\
\text { at } x=r_{p} b & u_{x}=0
\end{array}
$$

Thus,

$$
u_{x}=\frac{\delta}{2}\left(1-\frac{x}{r p b}\right)^{0.125}
$$

and

$$
\varepsilon_{x}=\frac{\delta}{16\left(r_{p} b\right)^{0.125} x^{0.875}}
$$

If the materials strain hardening behavior is idealized as linear, one can easily solve for the change in $\sigma_{x}$ due to a plastic strain, $\varepsilon_{x}$ : 


$$
\begin{aligned}
\Delta \sigma_{x} & =\varepsilon_{x} E_{p} \\
& =\frac{E_{p} \delta}{16\left(r_{p} b\right)^{0.125} x^{0.875}}
\end{aligned}
$$

where $E_{p}$ is the plastic modulus.

Equation (57) can be substituted into Equation (48) for effective stress $\left(x=x_{c}, \delta=\delta_{C}\right)$ :

$$
\bar{\sigma}_{c}=\sigma_{f}-\sigma_{x}{ }^{0}+\frac{E_{p} \delta_{c}}{16\left(r_{p} b\right)^{0.125} x_{c}^{0.875}}
$$

Thus, the relaxation slope is given by

$$
\frac{K}{2 A}=\frac{E_{p}}{16\left(r_{p} b\right)^{0.125} x_{c}^{0.875}}
$$

Experimental values of relaxation slope and equation (59) are plotted against ligament length in Figure 58. The plastic modulus was estimated by constructing a line, tangent to the flow curves in Figure 51 , which passed through the origin. For the normalized steel, $E_{p}$ was approximately $1750 \mathrm{MPa}$. The downward trend of relaxation slope with ligament length in Figure 58 is steeper for the data than for the prediction. All experimental values are within 10-50 percent of the prediction. This agreement is reasonably good considering the simplicity of the model and the relatively large scatter in the fracture toughness data used to compute relaxation slopes. 


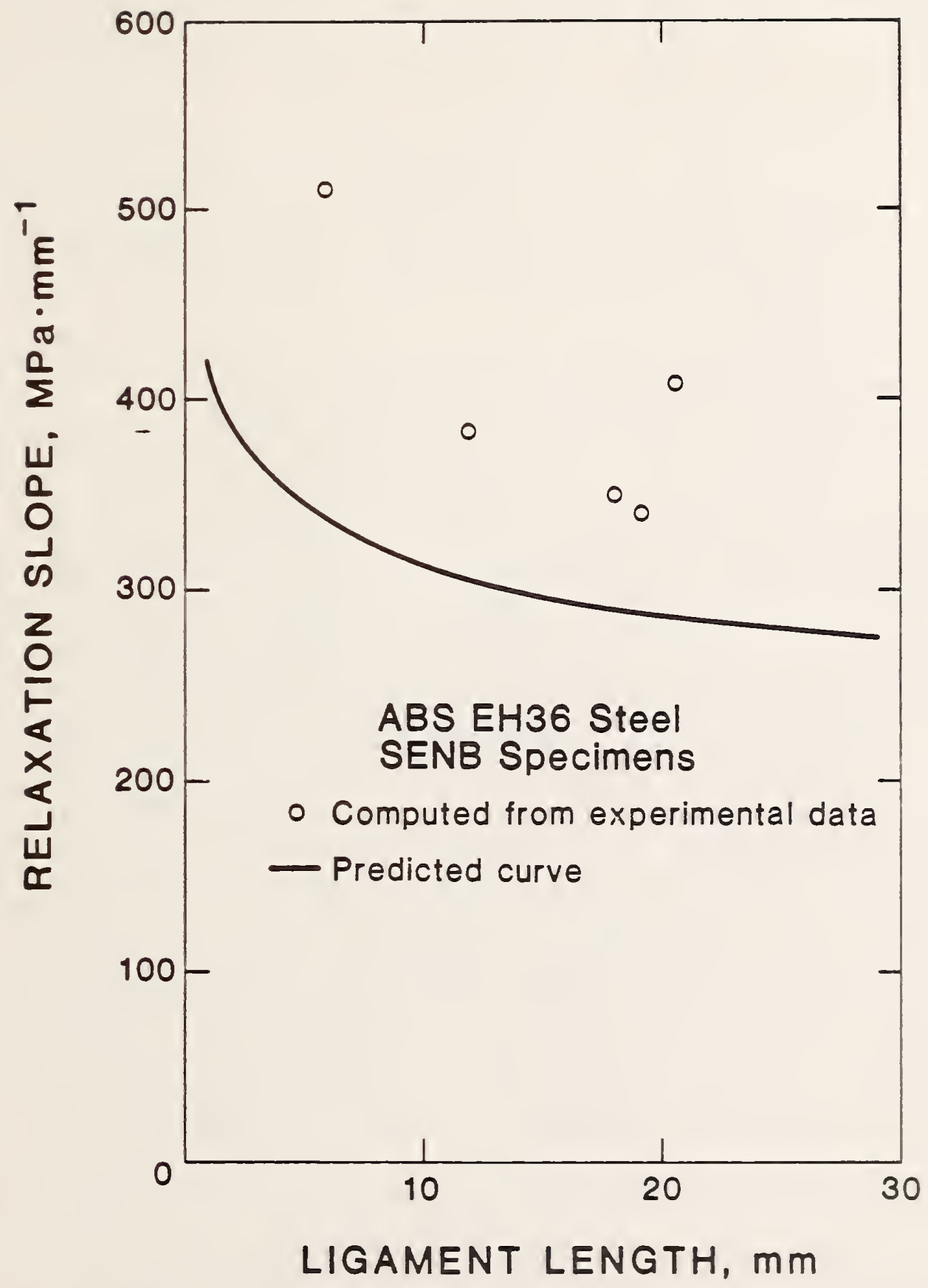

Figure 58. Predicted and experimental relaxation slope as a function of 1 igament length for ABS EH36 steel SENB specimens. 
It would be wise to pause at this point to further discuss the physical basis of this model. The picture presented in Figure 53 is one where an initially sharp crack blunts (at a constant $\sigma_{y y}$ ) and the stress in the $x$ direction relaxes by $\Delta \sigma_{x}$. The physical rationale of the above derivation is somewhat different from this concept, although the basic results are the same.

If a specimen containing a sharp crack is loaded $\left(\sigma_{y y}=\sigma_{f}\right.$ at $x=$ $x_{c}$ ) at a low temperature such that plastic deformation at the crack tip where minimal, the stress in the $x$ direction would be $\sigma_{x}^{0}$ at $x_{c}$. If a sharp-cracked specimen is monotonically loaded at a higher temperature, the crack tip blunts before $\sigma_{y y}$ reaches $\sigma_{f}$ because the material's flow stress is too low to maintain the sharp-crack configuration at high stresses. Therefore $\sigma_{x}$ will be less than $\sigma_{x}^{0}$ and constraint will be lower for the blunt crack. If this blunt-crack configuration is then plastically deformed in the $x$ direction to produce the sharp-crack configuration, $\sigma_{x}$ (at a constant $\sigma_{y y}$ ) would increase by $\Delta \sigma_{x}$ due to strain hardening. The ratio of principle stresses required to maintain the sharp-crack configuration should only be a function of specimen geometry and elastic properties. Therefore the value of $x$-stress for this configuration should be constant for $\sigma_{y y}=\sigma_{f}$; i.e., $\sigma_{x}+\Delta \sigma_{x}=\sigma_{x}^{0}$, regardless of temperature or prior strain history.

Although the above model is a refinement of the initial linear-elastic spring analog, both analyses predict a linear dependence of critical effective stress on critical CTOD. In the next section, 
this linear relationship is used to derive an equation for ductile-to-brittle transition curves.

\subsubsection{Predicting Transition Curves}

Equations (52) and (59) allow one to calcuate the slope and intercept of a material's constraint relaxation curve. This information, along with material-flow property data, make it possible to predict fracture toughness $\left(\delta_{c}\right)$ as a function of temperature in the ductile-to-brittle transition region.

Figure 59 schematically shows how one can obtain a critical CTOD value at a given temperature by superimposing material flow curves on the effective stress-CTOD plot. The stress-strain curve for an element $x_{c}$ ahead of the crack tip can be converted to a stress-CTOD curve by means of equation (42). Critical conditions occur at the point where the flow curve crosses the relaxation curve. The ductile-to-brittle transition curve can be constructed by plotting flow curves at the various temperatures on the relaxation plot and determining the corresponding $\delta_{C}$ values (see Fig. 59). Varying specimen geometry causes the relaxation curve to shift up or down (see Fig. 55). A downward shift of the relaxation curve causes a shift in the ductile-to-brittle transition to higher temperatures.

The above graphical analysis can be expressed in equation form. The material's flow behavior is assumed to follow power-law hardening: 


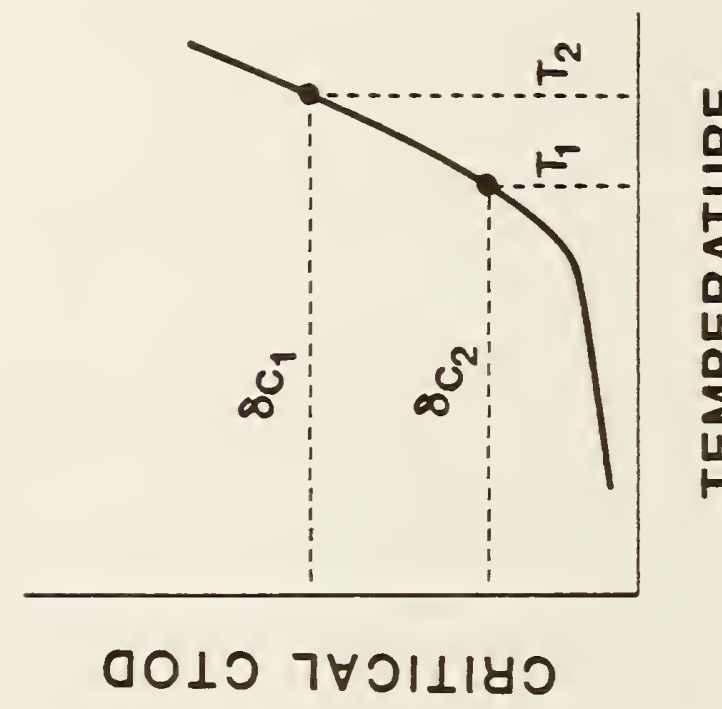

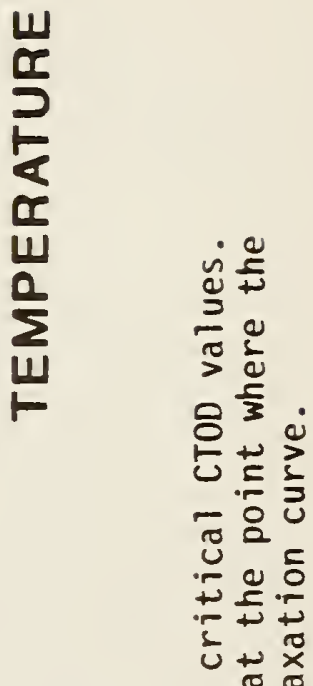

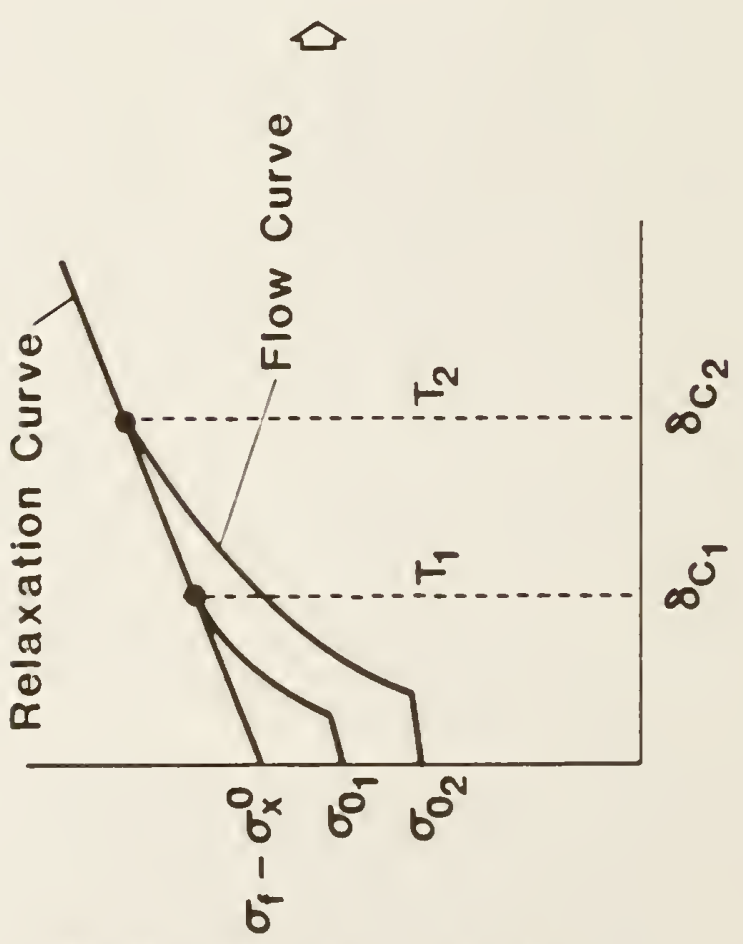

पै $=\overline{0}$

ह

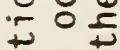
$\approx \simeq$ E气 E.厂 西? 원 워 정정

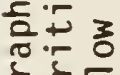
- 1 눤든 0 ถึ่

造

SS $\exists \forall \perp S \exists \wedge I \perp 0 \exists \unlhd \exists \exists$ 


$$
\bar{\sigma}=\sigma_{0}+\alpha \varepsilon^{n}
$$

Using equation (41) to solve for strain yields

$$
\bar{\sigma}=\sigma_{0}+\beta \delta^{m}
$$

where $\beta=\frac{0.10 \alpha}{x^{0.875}}$

and $m=0.875 n$.

For critical conditions, equation (60) is set equal to equation (58):

$\bar{\sigma}_{c}=\sigma_{0}+\beta \delta_{c}^{m}=\sigma_{f}-\sigma_{x}^{0}+\frac{E_{p} \delta_{c}}{16(\mathrm{rpb})^{0.125 x_{c}{ }^{0.875}}}$

Thus,

$$
\beta \delta_{c}^{m}-\frac{E_{p} \delta_{c}}{16\left(r_{p} b\right)^{0.125} x_{c}^{0.875}}=\sigma_{f}-\sigma_{x}^{0}-\sigma_{0}
$$

Equation (62) expresses fracture toughness (in the transition region) as a function of three stresses. The fracture stress is a material property, independent of temperature and geometry. The yield stress is independent of geometry but is temperature dependent. The geometry dependence is contained in the initial lateral stress, $\sigma_{x}^{0}$. This stress governs the temperature at which the upturn in the toughness-temperature curve occurs, i.e., the border between the lower shelf and the transition region. The factors on the left side of 
equation (62), such as strain hardening behavior and ligament length, govern the slope of the transition curve.

Several transition curves for normalized steel SENB specimens were computed by graphical means (see Fig. 59). These curves are shown in Figures 60-62. The relaxation slope and intercept used to construct the computed curves were taken from Table 10. The computed curves in Figures 60 and 61 are compared with the corresponding experimental data. These curves can be thought of as best-fit functions through the data. Figure 62 compares the computed curves for three SENB geometries. These curves represent the data presented in Figure 25 . The deepest notched geometry experiences the most rapid constraint relaxation because the ligament is shorter. This results in the steepest transition. That is why two of the curves cross in Figures 25 and 62.

The predicted curves were obtained by computing the slopes and intercepts of the corresponding relaxation curves from equations (52) and (59). The predicted curve in Figure 60 lies about $10-20^{\circ} \mathrm{C}$ to the right of the computed curve which passes through the data. This could be considered a reasonable prediction of the fracture toughness data. However, the predicted curve in Figure 61 lies well to the right of the experimental data. This curve yields overly conservative estimates of fracture toughness.

The reason for the poor agreement in Figure 61 can be understood by comparing predicted and experimental values of $\sigma_{x}^{0}$ in Figure 56. At low $L$ values the agreement is reasonably good but the predicted and 


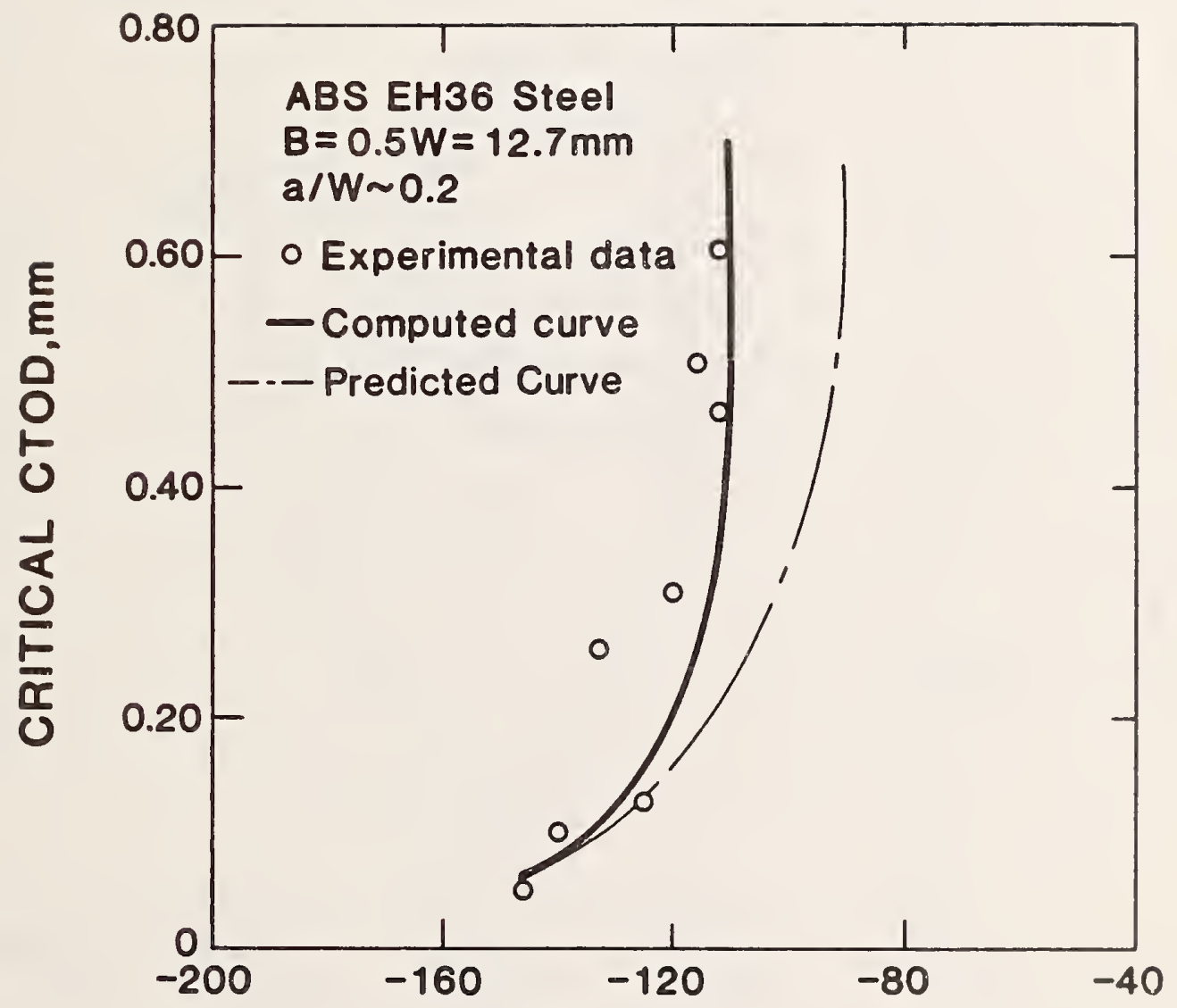

\section{TEMPERATURE, ${ }^{\circ} \mathrm{C}$}

Figure 60. Comparison of computed and predicted transition curves with experimental data; $\mathrm{ABS}$ grade $\mathrm{EH} 36$ steel SENB specimens, $B=0.5 W=12.7 \mathrm{~mm}, a / W=0.2$. 


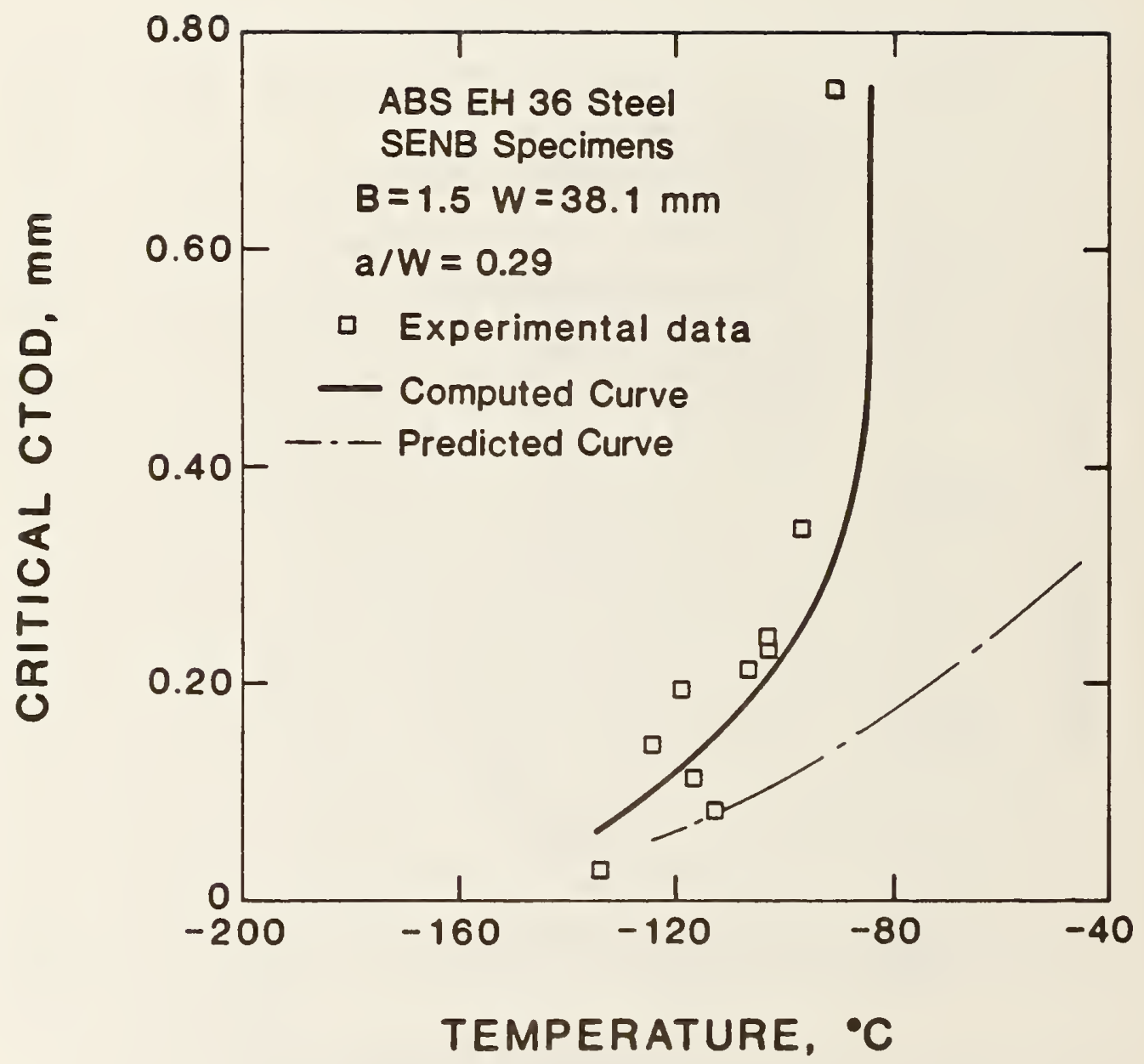

Figure 61. Comparison of computed and predicted transition curve with experimental data; ABS grade EH36 steel SENB specimens, $B=1.5 \mathrm{~W}=38.1 \mathrm{~mm}, \mathrm{a} / \mathrm{W}=0.29$. 


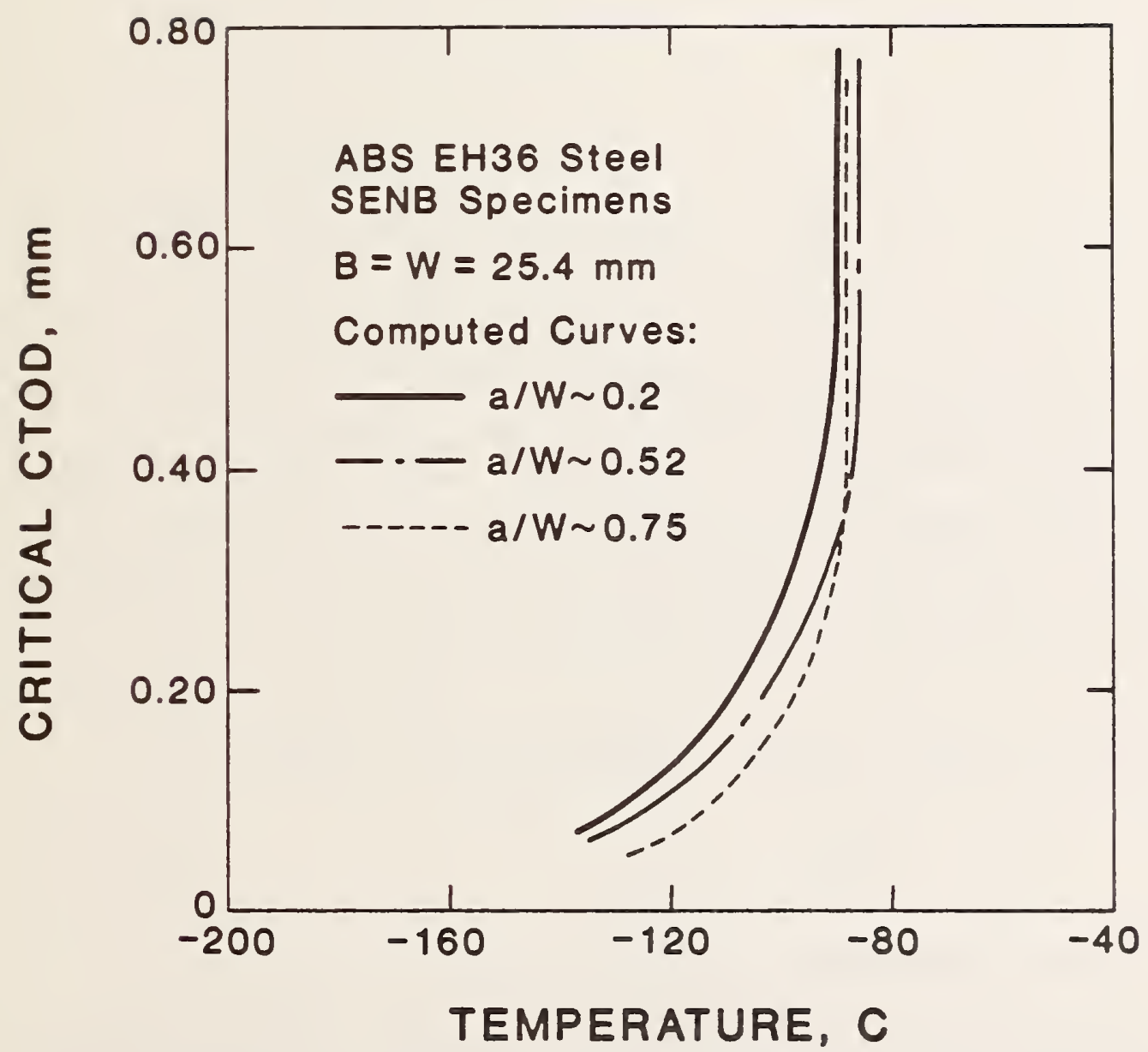

Figure 62. Computed transition curves for SENB specimens as a function of crack length. 
experimental values diverge at high $L$ values. That is why the agreement between prediction and experiment is much better in Figure $60(L \cong 1.35)$ than in Figure $61(L \cong 1.5)$.

The predicted transition curve in Figure 61 is much less steep than the constructed curve. This is mainly due to the fact that the temperature dependence of flow stress is less steep at higher temperatures (see Figs, 20 and 21). This results in a more gradual ductile-to-brittle transition at higher temperatures.

The model in its present form is not suitable for prediction purposes, as evidenced by the poor agreement between experiment and prediction in Figure 61 . The models which estimate $\sigma_{x}^{0}$ and the relaxation slope apparently need to be refined. The predicted curves are highly sensitive to variations in the initial lateral stress. However, these curves are not nearly as sensitive to the relaxation slope. Therefore, some of the predicitive capability might be salvaged by resorting to an empirical correlation between $\sigma_{x}^{0}$ and $L$. A linear regression fit of the data in Figure 56 yielded the following equation.

$$
\frac{\sigma_{x}^{0}}{\sigma_{f}}=0.183 \mathrm{~L}+0.242
$$

\subsubsection{Application to Structures}

With the empirical correlation between $\sigma_{x}^{0}$ and $L($ en. 63), the above analys is can be used to predict the ductile-tc-brittle transition curves of a structure. This structural application was essentially the original objective of this study. In the following example the model is 
used to predict the transition behavior of a hypothetical structure of ABS grade EH36 steel.

If one is to predict fracture toughness in the transition region for a structure of a given material using equation (E2) (or the equivalent graphical method), the following information is rißeded:

1) Yield stress versus temperature data

2) Material flow properties

3) Fracture stress

4) Critical distance

5) Ligament length

6) Initial lateral stress.

The first three items can be obtained from uniaxial tensile tests at various temperatures. The critical distance can be obtained fron: a lower shelf $K_{I c}$ test and Tracey's [82] finite element solutions. The last two items in the above list are dependent on the configuration of the specific structure.

For the purpose of this example a value of $150 \mathrm{~mm}(5.9 \mathrm{in})$ was assumed for $b$. The relaxation slope is not highiy sensitive to this value since the exponent on $b$ in equation (62) is small.

For conditions of optimal constraint, the value of $L$ is approximately $\sqrt{3}$. However, this value may be overly conservative for a typical structure. A very deep crack would be required to produce optimal notch constraint. Typical structural fiaws tend to be relatively shallow. An $L$ value of 1.5 may be more realistic. 
Values of $\sigma_{x}^{0}$ for ABS EH36 steel were computed from equation (63) assuming $L$ values of 1.5 and $\sqrt{3}$. The slope of the relaxation curve was calculated from equation (59). The ductile-to-brittle transition curves were constructed by means of the graphical method.

Figure 63 shows the predicted transition curves for the two assumed $L$ values. A typical computed curve for an SENB specimen is included for comparison. The predicted temperature shift between the structure and laboratory specimen is small at low toughness and large at high toughness values. The predicted structural curves are less steep than the specimen curve for two reasons. First, the relaxation slope for a large section is lower. Second, as mentioned previously, the temperature dependence of flow stress is not as steep at higher temperatures.

The structural prediction for an assumed $L$ value of 1.5 looks reasonable. Unfortunately, there are no large section data available to compare with this prediction.

Another problem is that the existing model is only applicable for loading in bending. Most structures undergo tensile loading or a combination of bending and tension. Since pure bending is the worst-case situation with respect to constraint, the present analysis would give conservative results. It remains to be seen whether this conservatism is reasonable or excessive.

All things considered, the predictive capability of the present analyses for structures is definitely suspect. Obviously, further refinements are needed. Predictions have been made in Sections 3.4.6 


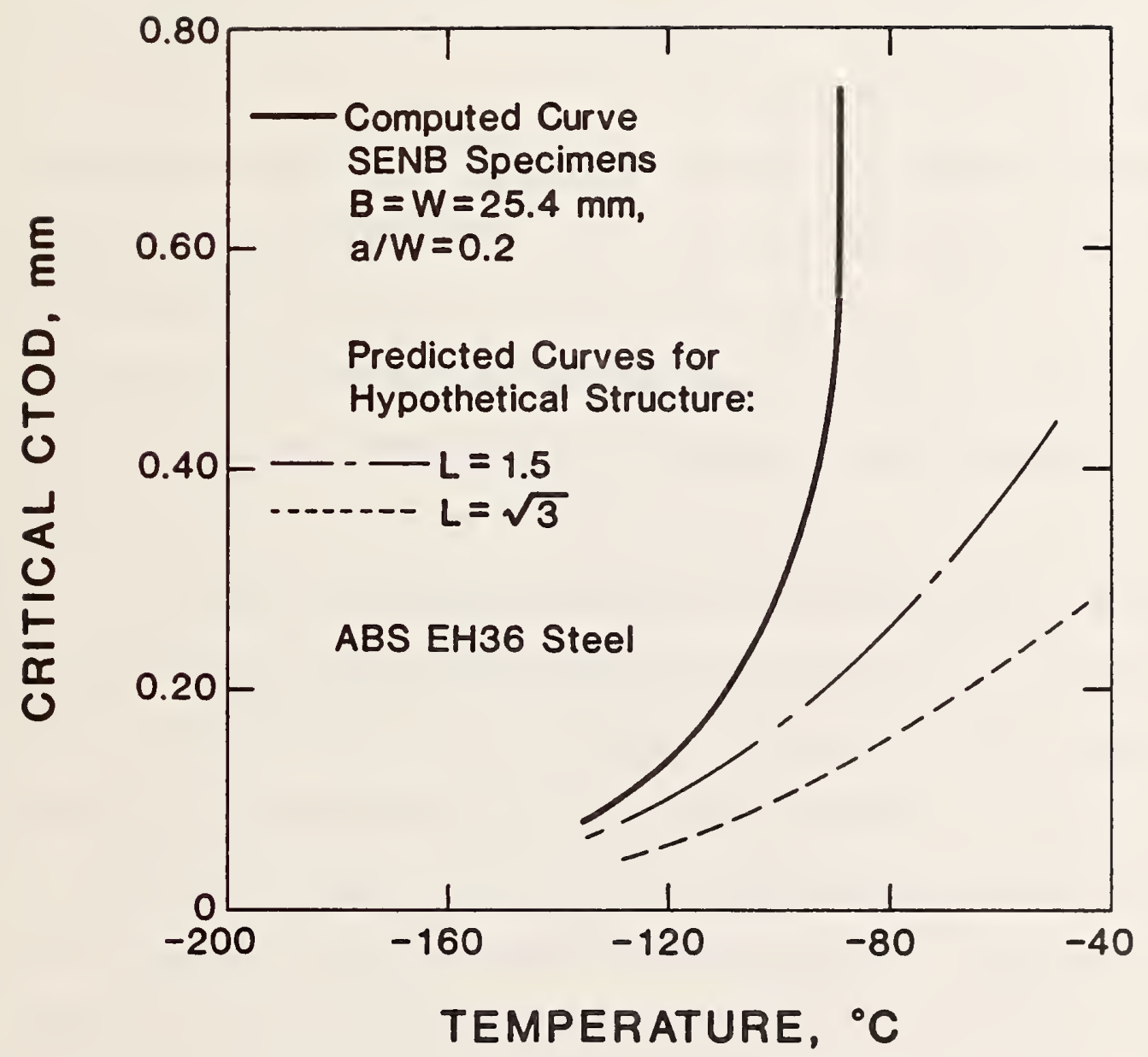

Figure 63. Predicted transition curves for a hypothetical structure of ABS EH 36 steel compared with a computed curve for an SENB specimen. 
and 3.4.7 simply to demonstrate the potential of a model such as this, once its accuracy can be assured. Although the model in its present form does not agree with experimental data as well as one would hope, it does provide valuable insights into material behavior.

\subsubsection{Application to Other Materials}

The model for the ductile-to-brittle transition was used to predict transition curves for the commercially pure iron. It was reasoned that if this analysis could be successfully applied to both the C-Mn steel and the pure iron, the analysis could be used to predict the position and shape of transition curves for most other low-carbon structural steels.

As stated in Section 3.4.7, six items are needed to predict transition curves. Since tensile data was obtained for the pure iron, the first three items on the 1 ist in Section 3.4 .7 were readily available. However, it was not possible to determine an accurate value for critical distance because none of the fracture toughness tests on this material yielded a valid $K_{I c}$ value. Values of $0.15,0.30$, and $0.45 \mathrm{~mm}$ were assumed for $x_{c}$; this correpsonds to approximately 1,2 , and 3 grain diameters, respectively. The initial lateral stress was estimated according to a rationale described below.

Two assumptions were made for the estimation of $\sigma_{x}^{0}$ for pure iron. First it was assumed that $L$ is constant for a constant geometry of the two materials. The notch constraint factor is insensitive to 
temperature but is a function of specimen geometry (Figs. 28 and 29). This suggests that $L$ is primari?y controlled by a material's elastic properties; the flow properties should be of secondary importance. If this is true, specimens of two materials with the same geometry and elastic properties should have the same $L$ value. Second, it was assumed that equation (63) is valid for both materials. Thus, the two specimens should also have the same $\sigma_{X}^{0} / \sigma_{f}$ ratio. The average fracture stress for the pure iron was $627 \mathrm{MPa}$ (Fig. 46). Thus $\sigma_{x}^{0}=316 \mathrm{MPa}$ for $B=25.4 \mathrm{~mm}$ and $a / W=0.2$.

The assumption that $L$ is not material sensitive was investigated. Figure 64 is a plot of nondimensional load versus displacement for normalized steel and pure iron SENB specimens with the same geometry. These two curves agree reasonably well at high displacements but the agreement is not as good near net-section yield. Much of the difference is due to the fact that the normalized steel has a sharper yield point than pure iron. Apparently, the value of $L$ is slightly dependent on flow properties since a finite amount of plastic deformation usually precedes ligament yielding. However, the disagreement between the two curves is not bad considering that the flow properties of these two materials are vastly different. The notch constraint factor for a given specimen geometry probably remains relatively constant for a wide range of steels.

Constraint relaxation curves and transition curves were computed from experimental fracture toughness results. This was done to compare 


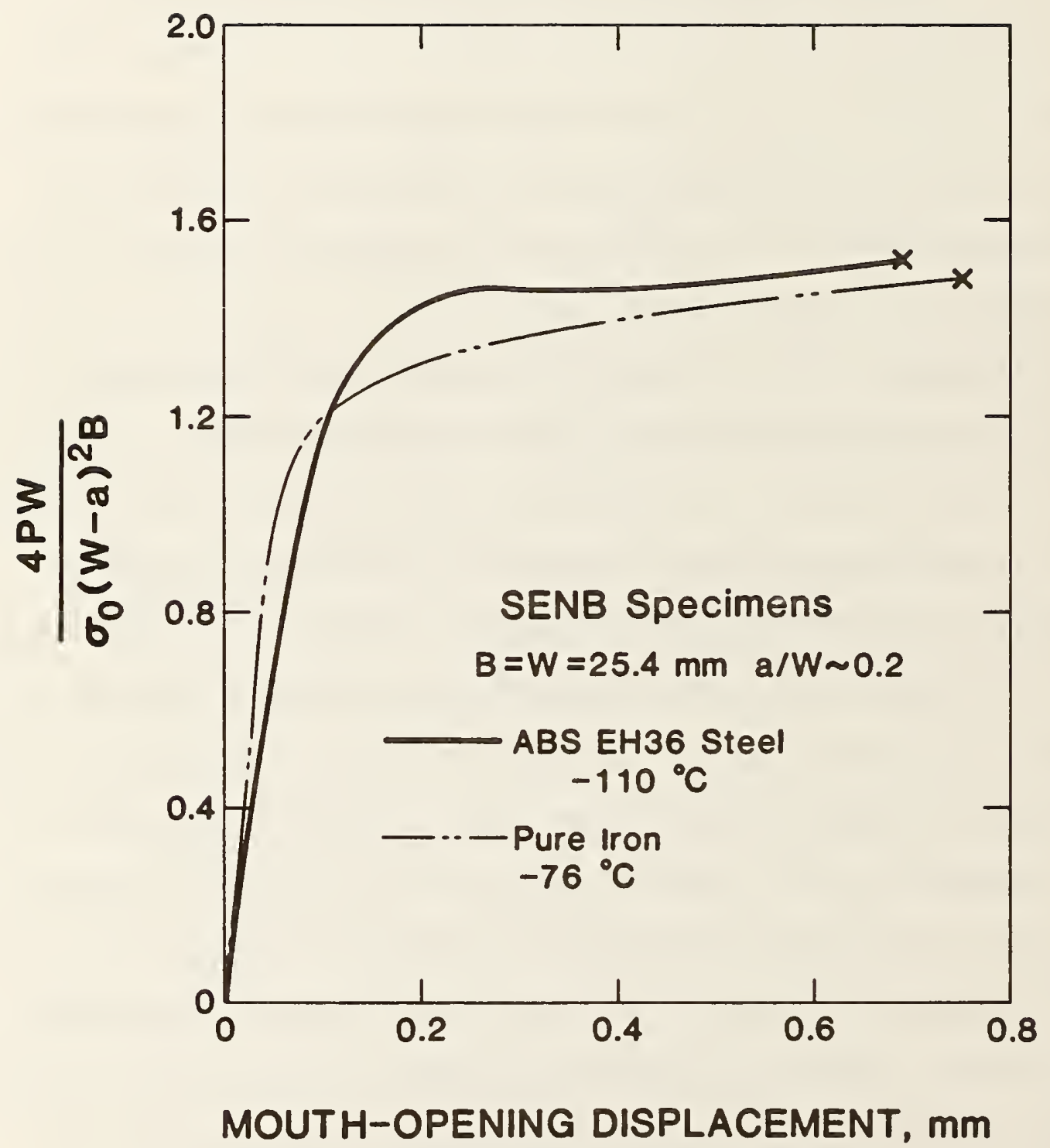

Figure 64. Nondimensional-load-versus-displacement curves at a constant SENB specimen geometry for pure iron and ABS EH36 steel. 
predicted and experimental results. Also, the effect of the assumed $X_{C}$ value could be assessed. Figure 65 shows that the strain-hardening behavior of this material is not sensitive to temperature over the range of interest. The flow curves for pure iron were converted to stress/CTOD curves corresponding to the three assumed critical distances. These curves are plotted in Figure 66. Critical effective stress was measured from experimental data according to the procedure outlined in Section 3.4.2.

The relaxation curves for the three assumed $X_{c}$ values are shown in Figure 67. Both slope and intercept are functions of the assumed critical distance. The expected intercept, which is marked on the ordinate of Figure 67 , was determined by assuming that the $\sigma_{x}^{0} / \sigma_{f}$ ratio is the same as that of the normalized steel specimens with the same geometry. This predicted intercept agrees well with the actual experimental intercepts.

Ductile-to-brittle transition curves were constructed graphically according to the procedure illustrated in Figure 59. These curves are shown in Figure 68. The constructed curves are not strongly affectly by the assumed $X_{c}$ value. This procedure for computing best-fit curves through fracture toughness data is apparently not sensitive to errors in $x_{c}$.

The relaxation slope for pure iron was predicted from equation (59) as a function of distance ahead of the crack tip. The plastic modulus of this material was approximately $750 \mathrm{MPa}$. Figure 69 compares the 


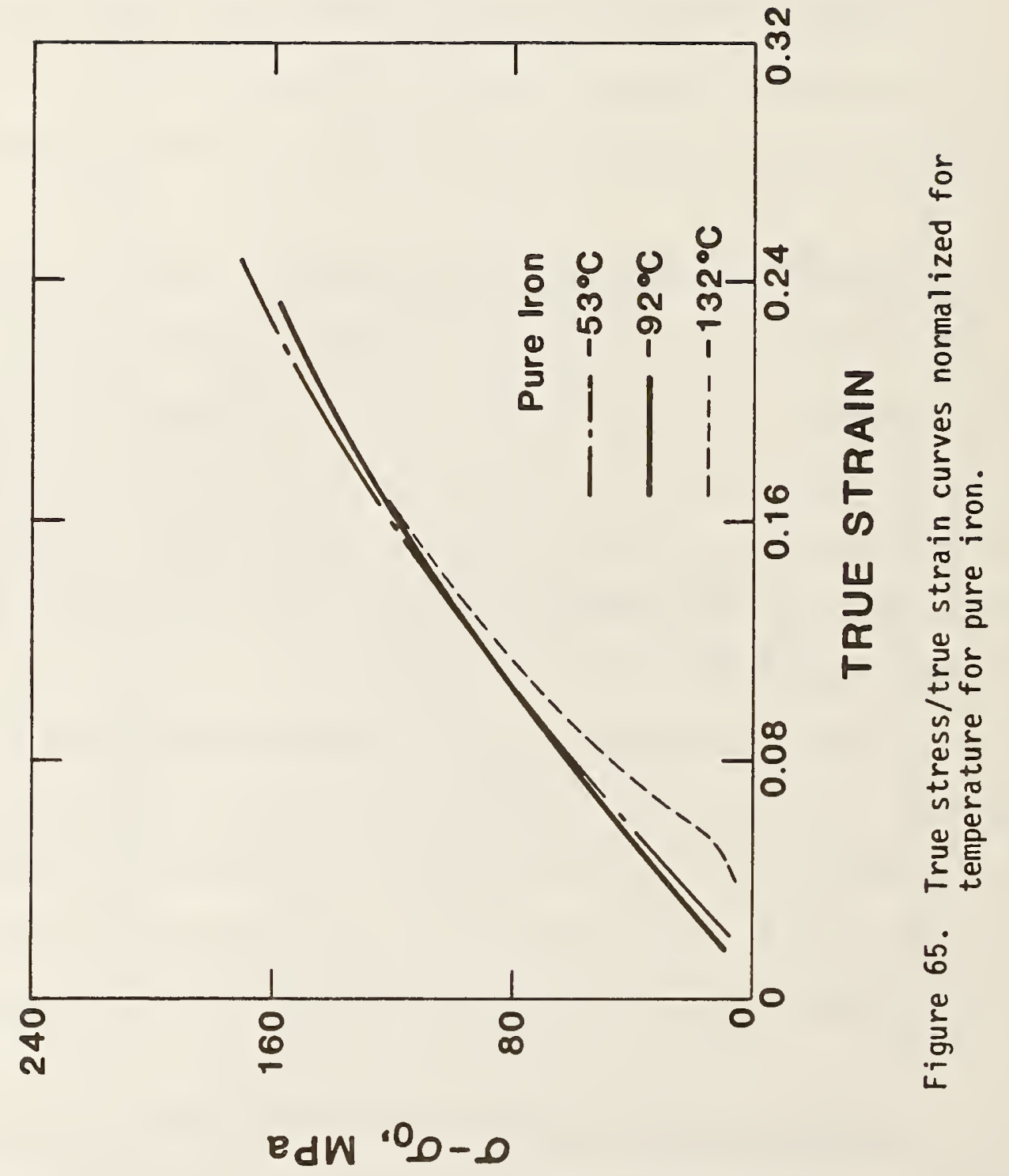




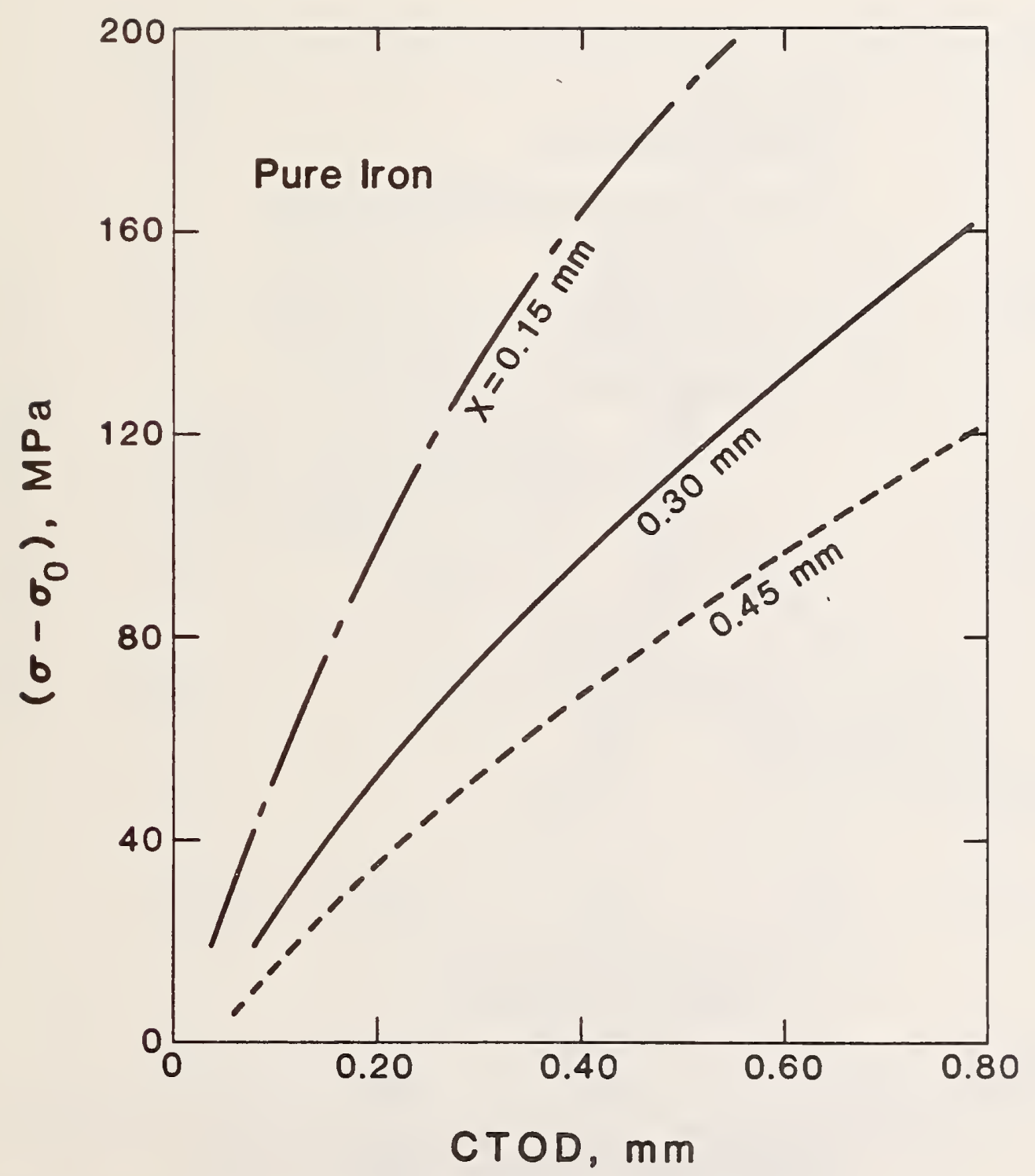

Figure 66. Flow behavior of pure iron ahead of a crack tip as a function of CTOD and distance. 


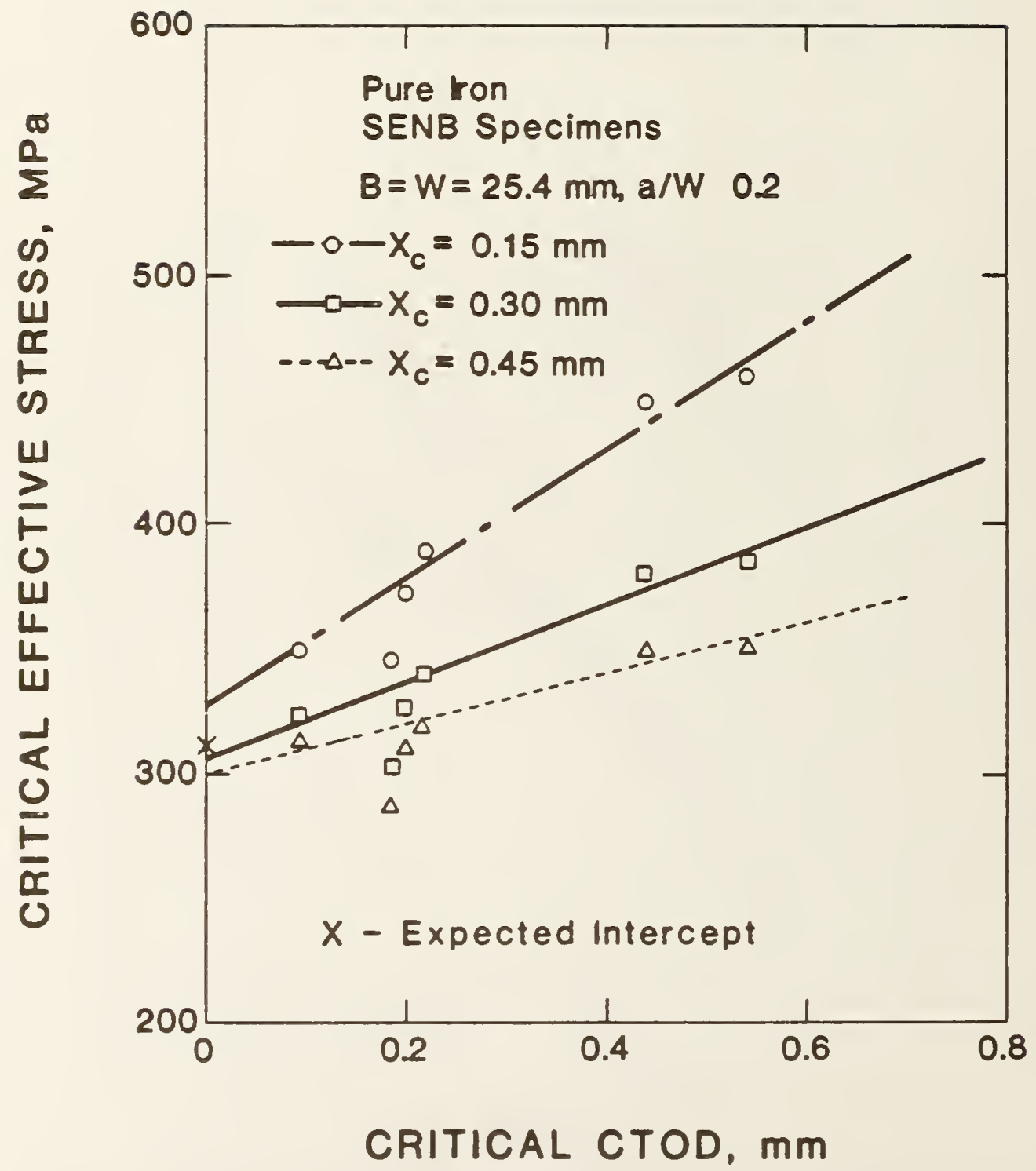

Figure 67. Critical effective stress as a function of critical CTOD at three assumed values for critical distance in commercially pure iron SENB specimens. 


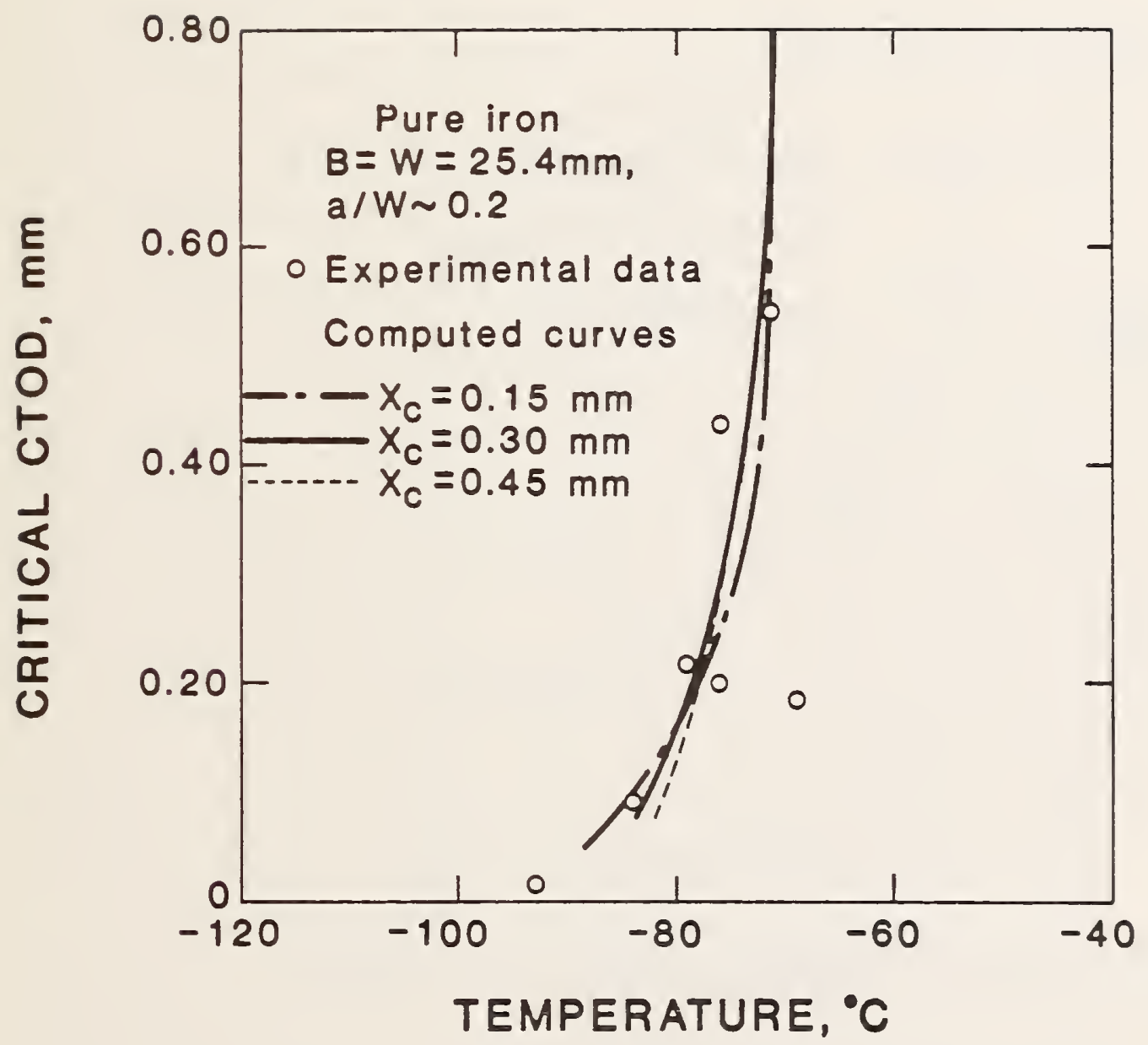

Figure 68. Computed transition curves at three assumed critical distances compared with experimental data for commercially pure iron SENB specimens. 


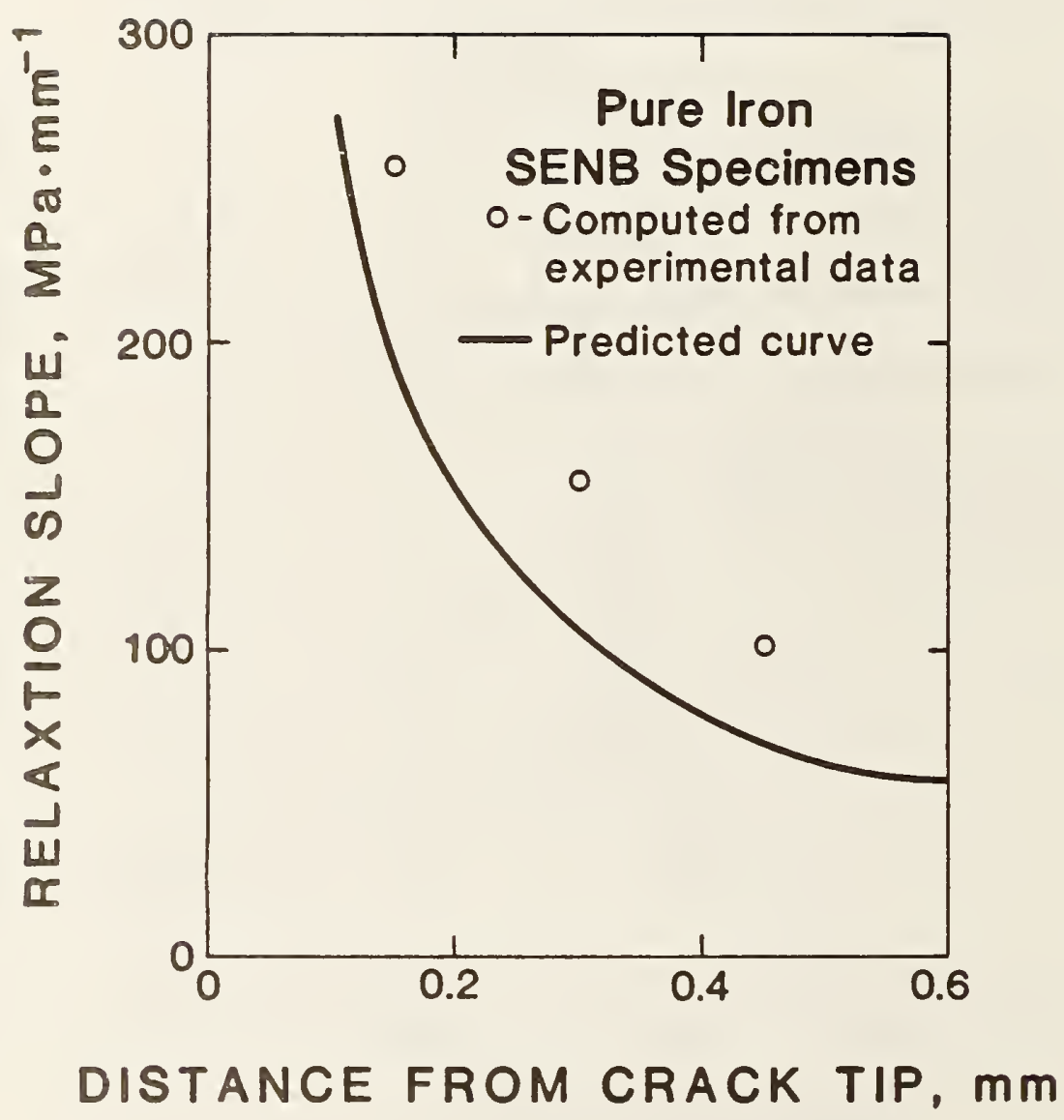

Figure 69. Predicted and experimental relaxation slope as a function of distance from the crack tip for commercially pure iron. 
predicted curve with actual values of slope obtained from Figure 67. As in Figure 58, the agreement between experimental and predicted relaxation slopes is reasonably good.

Using the predicted intercept and relaxation slopes, transition curves were constructed graphically for the three assumed critical distances. These predicted curves are compared with experimental data in Figure 70. The best agreement was obtained for an assumed critical distance of $0.45 \mathrm{~mm}$ although the curve for $X_{c}=0.30 \mathrm{~mm}$ agrees relatively well with the data.

A value of $0.15 \mathrm{~mm}$ ( 1 grain diameter) is probably an underestimate of the average critical distance in the pure iron. One would expect that more than one grain ahead of the crack tip would have to be sampled to find a particle large enough to initiate fracture. However, one data point in Figure 70 does coincide with the $x_{c}=0.15 \mathrm{~mm}$ curve. It is likely that a large inclusion was near the crack tip in this specimen.

The predicted curves in Figure 70 shift to higher temperatures with decreasing critical distance. One could thus obtain a lower-bound curve for a given material by assuming a lower-bound value for $x_{c}$.

The model developed in this investigation seemed to work very well for predicting ductile-to-brittle transition curves for the pure iron. The accuracy of the predictions cannot be fully ascertained because an accurate value for the critical distance was not available. However, $0.30-0.45 \mathrm{~mm}$ is certainly a reasonable range for $x_{c}$. 


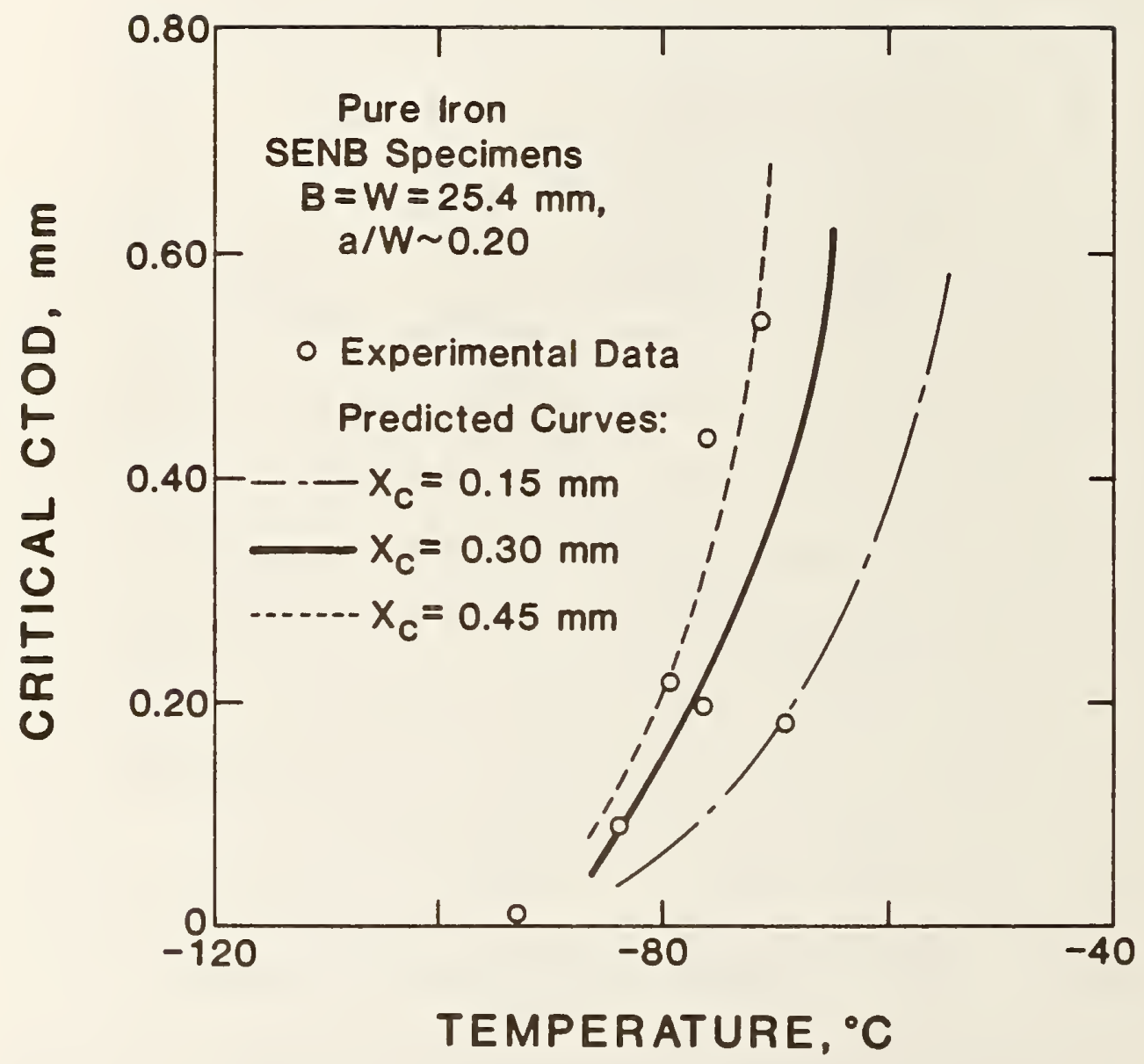

Figure 70. Predicted transition curves at three assumed critical distances compared with experimental data for commercially pure iron SENB specimens. 
Although, as stated previously, the accuracy of prediction from the model is often unacceptible, the model seems to work well for describing the ductile-to-brittle transition behavior of both materials. Therefore, it can probably be successfully applied to most structural steels.

\subsection{Constraint Versus Statistical Models}

As stated in Section 1.4.2, there are two explanations given for size effects on fracture toughness in the transition region. The traditional explanation is that smaller specimens lose constraint. The present analysis utilizes this concept. The second explanation for size effects was recently proposed by Landes and Shaffer [60]. They maintain that these effects can be explained by a statistical model for cleavage fracture. In a large specimen, where a larger volume of material along the crack front is sampled, the probability of finding a region of low toughness (e.g., a large grain boundary carbide) along the crack front is higher. Therefore, the toughness of a large specimen should be lower than the average toughness of a population of smaller specimens.

There is somewhat of a controversy over whether constraint or statistical effects are the dominant factor in size effects on fracture toughness. A discussion of this topic in light of the present results is given here. Both arguments have some validity. It is hoped that this section will help to put these different approaches into perspective. 
Both constraint and statistics could conceivably contribute to size effects. The relative contribution of each of these factors can be qualitatively understood by examining the variables that affect their magnitude.

\subsubsection{Factors that Affect Constraint}

Constraint is lowest for thin specimens. As specimen thickness increases, constraint increases until plane strain conditions are reached. At this point further increases in thickness have little or no effect on constraint. The thickness at which plane strain is reached depends upon material flow properties. For a low-strength steel, a thicker specimen is required for near-plane strain conditions. For a given applied stress intensity, the plastic zone is larger in a lower strength material (eq. 9). As seen in Section 3.4, increased plasticity results in a constraint relaxation.

In addition to thickness, the crack length and type of loading affect constraint. Deeper cracks produce additional constraint in bend specimens. For very deep cracks, constraint is initially higher but when the remaining ligament is short, constraint relaxes quickly with plastic flow. Also, bending produces more constraint in deep-noteched specimens than tensile loading. 


\subsubsection{Factors that Affect Statistical Shifts in Toughness}

As in the constraint concept, the statistical model dictates that a material's mean fracture toughness at a given temperature (in the transition region) must decrease with increasing thickness. The statistical model is also similar to the constraint argument in that statistical effects must saturate at a certain thickness. Once a large enough volume of material is sampled, further increases in sample size would have no effect because larger samples would not contain any additional microstructural features.

The major factor which would likely control the saturation thickness for statistical effects is the microstructure. For example, a homogenious microstructure would have a small saturation thickness. A material that contains a low volume fraction of large grain boundary carbides would exhibit statistically-related size effects up to a relatively large thickness because a large volume of material would need to be sampled in order to insure that the process zone ahead of the crack tip contained at least one of the large particles.

The grain size of a material could affect the saturation thickness since the number of grain boundary carbides or other fracture-triggering particles in a given volume of material is related to the number of grains sampled in that volume. The grain size effect is illustrated in the following example. Assume for this example that the volume of material along the crack front that can contribute to fracture initiation is contained in a circular cylinder with a radius of $0.2 \mathrm{~mm}$ 
and a length equal to the thickness of the fracture specimen. For an average grain diameter of $0.03 \mathrm{~mm}$, approximately $10^{5}$ grains would be sampled in a $25 \mathrm{~mm}$ thick specimen. If the grain diameter is doubled, a specimen $200 \mathrm{~mm}$ thick would be required to sample $10^{5}$ grains along the crack front. Thus, the saturation thickness would be smaller for fine-grained materials .

Unlike constraint, the statistical size effects are not influenced by crack length. If the crack length is varied at a constant thickness, the volume of material along the crack front remains constant. Any shifts in the transition that occur as a result of varying the a/W ratio must be attributable to constraint effects. Similarly, transition shifts between bending and tensile loading can be attributed entirely to constraint effects.

\subsubsection{Determining the Cause of Transition Shifts}

The material properties that can determine the magnitude of constraint and statistical effects were outlined above. With this information it is often possible to determine the dominant cause of size effects on fracture toughness of a given material.

Landes and Shaffer $(60)$ used an ASTM A471 NiCrMoV rotor steel with a yield strength of $765 \mathrm{MPa}$ (111 ksi) for their statistical study. They performed fracture toughness tests on $I T$ and $4 T$ compact tension (CT) specimens. The $1 T-C T$ and $4 T-C T$ specimens were $25.4 \mathrm{~mm}(1.0 \mathrm{in})$ and $102 \mathrm{~mm}(4.0 \mathrm{in})$ thick, respectively. For both $\mathrm{CT}$ geometries, $\mathrm{B}=\mathrm{a}=2 \mathrm{~W}$. 
Landes and Shaffer correlated transition shifts between the two geometries with a weakest-link statistical model. They assumed that the deep-notched IT-CT configuration contained a high degree of constraint and that the constraint in this specimen was equivalent to that in the 4T-CT geometry. This was probably a valid assumption since the material had a high yield stress. However, there would likely be a pronounced constraint difference between $1 T$ - and $4 T-C T$ specimens of a low'-strength mild steel.

The A471 steel used by Landes and Shaffer exhibited significant statistical effects but no differences in constraint were observed. In contrast, the ABS grade EH36 steel used in the present study exhibited significant constraint effects and minimal statistical effects. This material is a fine-grained nomalized steel with a yield stress approxmiately half that of the $A 417$ steel.

Figures 23-26 and 35 show that the ductile-to-brittle transition of the ABS EH36 steel shifts to higher temperatures by: 1) increasing specimen thickness, 2) decreasing uncracked ligament length in SENB specimens, and 3) changing loading from tension to bending in deep-notched specimens. The two latter transition shifts are due entirely to constraint effects for reasons discussed earlier. Figure 30 shows fracture toughness data for 25.4 and $76.2 \mathrm{~mm}$ ( 1.0 and $3.0 \mathrm{in}$ ) thick SENB specimens with $a / K=0.5$. The constraint in both of these geometries is approximately the same because corresponding values for the notch constraint factor, $L$, are equal. Thus, any observed 
transition shifts would be due to statistical effects. However, the data for the thick specimens all lie well within the scatter band of the $B=25.4 \mathrm{~mm}$ data. Thus, it can be concluded that for ABS EH36 steel, statistical effects are saturated at or below B $=25.4 \mathrm{~mm}$.

Of all the specimen geometries tested in this study, only one SENB configuration has a thickness less than $25 \mathrm{~mm}$. Figure 23 shows a definite transition shift from $B=12.7$ to $25.4 \mathrm{~mm}(\mathrm{a} / \mathrm{W}=0.2)$. Figure 29 shows that notch constraint is elevated in the thicker specimen. However, this does not prove that there are no statistical contributions to the transition shift. Some evidence for the hypothes is that statistical effects are minimal in the thin specimens is in Figure 56 where $\sigma_{x}^{0}$ is plotted against $L$ for five SENB geometries. In four out of five geometries, statistical effects are saturated because $B \geq 25.4 \mathrm{~mm}$. If statistical considerations significantily affected the transition shift between $B=12.7$ and $25.4 \mathrm{~mm}$, the measured value of $\sigma_{x}^{0}$ for $B=12.7 \mathrm{~mm}$ would be 1 ower than would be expected from constraint considerations alone; the data point for $B=12.7 \mathrm{~mm}$ in Figure 56 would lie below the trend established by the other data. However, the $B=$ $12.7 \mathrm{~mm}$ data lies on the same trend as the other data, suggesting that statistical effects are minimal.

Low strength ferritic materials can exhibit significant size effects due to loss of constraint in smaller specimens. Materials with inhomogenious or coarse-grained microstructures can experience statistically related size effects on toughness. The commercially pure 
iron used in this study falls into both of these categories. It has very low strength and its microstructure consists of large ferrite grains with a small volume fraction of large inclusions. Transition shifts with thickness in this material might be due to both constraint and statistical effects. Unfortunately, the relative contributions of each of these factors cannot be readily determined.

\subsubsection{Summary Comments}

Both constraint and statistical explanations for size effects on fracture toughness in the transition region have merit. The dominant factor depends on the material. Constraint effects are most pronounced in low strength steels. Statistical effects are at a maximum in materials with coarse-grained and/or inhomogenious microstructures. Sometimes a low strength material can also have a microstructure that is susceptible to statistical transition shifts. This was the case for the pure iron used in this study. Many weldments may also fit into this category. For these materials, an integrated mode? which considers both constraint and statistical effects is needed.

\subsection{Discussion of Results}

The original objective of this work was to develop a method for scaling size effects on fracture toughness in the ductile-to-brittle transition region of steels. Although a number of questions remain unanswered, substantial advances were made in this area. These advances 
are outlined below, along with the potential applications and limitations of the analyses developed here.

\subsubsection{Advances}

The experimental data obtained in this investigation provided a demonstration of constraint effects on fracture toughness. It is apparent from the results that, at least for some materials, size effects on fracture toughness can be attributed to constraint which is dependent on specimen size and geometry. Statistical effects may play a role in some materials but constraint is definitely a factor in low-strength steels.

A technique to experimentally measure crack-tip region constraint (CTRC) was developed during the course of this investigation. The first known quantitative measures of CTRC ever made are presented in this document. At a constant CTOD, constraint is lowest for a thin specimen with a shallow notch. Although this behavior was expected in light of prior work, all previous authors discussed constraint in purely qualitative terms.

The constraint measurements showed that CTRC decreases with crack blunting. This relaxation was modeled in this investigation. Although the model is relatively simple, the agreement with experimental data is quite good.

An equation for fracture toughness as a function of material-and geometry-dependent properties was derived from the constraint model. 
This equation can be used to predict ductile-to-brittle transition curves for low-carbon steels in various configurations. Material flow data, a lower-shelf fracture toughness value, and an estimate of the notch constraint in the configuration of interest are the only data required for this prediction. The accuracy of these predictions was not as good as one would hope but the analyses provided valuable insights into the physics of constraint and the ductile-to-brittle transition.

The model works well for describing the transition behavior of both ABS grade EH36 steel and commercially pure iron. This suggests that the description of the ductile-to-brittle transition inherent in the model may be universal. The analysis is probably applicable to most structural steels.

\subsubsection{Applications}

As stated above the model can be used to predict the fracture toughness of a structure if certain material data are available. However, a number of refinements are needed for the model to be reliable for predictive purposes. There are several different approaches to using this analysis for structural applications.

The most reliable approach would be to experimentaliy obtain accurate values for a 11 the parameters in equation (62). This would require uniaxial tensile tests, lower-shelf $K_{I c}$ tests and a realistic estimate of the notch constraint factor for a typical structural flaw. One could then compute the entire transition curve for this structure. 
The temperature shift approach may be suitable for material selection applications. It has been established that there is a shift in transition curves between small-scale test specimens and large structures. This temperature shift can now be quantified for a given situation. For example, take a structure which will operate at $-40^{\circ} \mathrm{C}$. Assume for this example that is has been determined that a critical CTOD value of $0.2 \mathrm{~mm}$ is required for this structure to be safe with respect to brittle fracture. The fracture toughness is to be measured from $25 \mathrm{~mm}$ thick SENB specimens with $\mathrm{a} / \mathrm{W}=0.2$, machined from a weldment of the candidate material. Equation (62) can be used to determine the temperature shift between the specimen and the structure at $\delta_{c}=0.2 \mathrm{~mm}$ and a service temperature of $-40^{\circ} \mathrm{C}$. If the temperature shift is determined to be $20^{\circ} \mathrm{C}$, the specimens must be tested at $-60^{\circ} \mathrm{C}$. The lower-bound toughness of the SENB specimens at $-60^{\circ} \mathrm{C}$ must be greater than $0.2 \mathrm{~mm}$ for the weldment to be suitable for the structural application.

The above methods require information about the flow behavior and lower-shelf toughness which might not be available in many cases. However, it is still possible to use the constraint model to make engineering estimates of a material's toughness. With a knowledge of the chemical composition, microstructure and approximate yield strength of a material, one can make estimates of the various parameters in equation (62) and then estimate a transition temperature for the material. This approach would not yield highly accurate predictions. 
However, it may be of use for determining whether a material will be brittle or ductile at a given temperature. For example this type of analysis, which involves no mechanical testing, could expedite material selection by screening out materials that are totally unsuitable for a given application. Expensive, time-consuming mechanical tests can then be performed on the more promising candidate materials.

\subsubsection{Limitations}

There is no question that the model developed in this investigation is an over-simiplication of the actual stress-strain behavior at the crack tip. For example, the model treats the plastic relaxation in the $x$-direction as a one-dimensional problem.

Although the analysis which predicts a relationship between initial lateral stress, $\sigma_{x}^{0}$, and notch constraint, $L$, yields values that are within 10-20 percent of experimental values, the predictions of the ductile-to-brittle transition curves were far too conservative when $\sigma_{x}^{0}$ is computed in this way. The author had to resort to an empirical fit between $\sigma_{x}^{0}$ and $L$. Although this correlation seemed to work well for specimens of both materials, it is not known how well the empirical relationship will work for larger section sizes.

The estimation of $\sigma_{x}^{0}$ for a large structure is just one problem that contributes to the uncertainties in structural predictions. Another problem is that the model in its present form cannot be applied to tensile loading. Fortunately the analysis, which is applicable to 
bending, would normally yield conservative predictions because bending is a worst-case loading condition.

Owing to the uncertainties mentioned above, the accuracy of structural toughness predictions is questionable. Large-scale experimental results are needed to compare with predictions. Unfortunately, large-scale fracture toughness testing is very expensive and requires an enormous test machine.

Another limitation of the analysis in its present form is that statistical effects are not taken into account. Although minimal in the ABS EH36 steel plate used in this study, statistical effects may be quite pronounced in inhomogenious materials such as weldments.

\subsubsection{Summary Comments}

Although there are a number of problems remaining, the results of this study are encouraging. A methodology has been developed that can be applied to the problem of scaling fracture toughness results. Several refinements of the analysis are needed to insure its accuracy in a wide range of applications. 


\section{SUMMARY AND CONCLUSIONS}

Specimen geometry has a drastic effect on fracture toughness in the ductile-to-brittle transition region. Increasing specimen thickness and/or crack length causes the transition to occur at higher temperatures. The geometry effects in ABS grade EH36 steel are attributed primarily to constraint effects. Thicker specimens produce a greater amount of stress triaxiality near the crack tip, thus elevating the flow stress locally. A higher flow stress tends to promote cleavage fracture by making it easier to reach the fracture stress. For specimens with relatively short ligaments the transition in bend specimens occurs at higher temperatures than for specimens loaded in tension. As ligament length is increased the transition behavior approaches that of tension specimens. For very short cracks or very short ligaments, constraint can be relaxed by plastic flow from the crack tip to the nearest free surface.

Crack-tip region constraint (CTRC) can be indirectly measured by means of the Ritchie-Knott-Rice fracture model and a crack-tip strain equation. The CTRC decreases with crack blunting. A simple model for this constraint relaxation predicts a linear relationship between critical effective stress and critical CTOD. This 1 inear relationship agrees well with experimental data.

The model for constraint relaxation can be used to predict ductile-to-brittle transition curves for a low-carbon steel as a 
function of geometry. This model can be applied to toughness predictions of large structures by assuming worst-case values for geometry-dependent parameters in the model.

The analysis in its present form was derived for SENB specimens and is not valid for tensile loading. However, bending is a worst-case loading situation; the present analysis should yield conservative (i.e., safe) predictions. The model does not take statistical effects into consideration. Although for many materials the statistical effects are negligible, materials with an inhomogeneous microstructure, such as weldments, may be highly susceptible to statistical size effects.

Future work should include refining the model to include statistical effects and tensile loading. Also, the accuracy of the analysis for structural predictions should be investigated withlarge-scale fracture toughness tests.

\section{ACKNOWLEDGMENTS}

I would like to express my deepest appreciation to the members of my doctoral committee, Drs. H. I. McHenry, G. R. Edwards, D. K. Matlock, S. R. Danie1, M. G. Dawes, S. B. Romberger, and S. D. Foreman. I especially thank $\mathrm{Dr}$. McHenry for the support and guidance he has given me these past three years. Special thanks are also due Dr. M. G. Dawes of the British Welding Institute who provided the initial inspiration for this work and has served as a mentor throughout this program. I am also grateful to the staff nembers of the Fracture and Deformation Division of the National Bureau of Standards who have shared their expertise and offered a great deal of helpful advice. Members of the NBS support staff who were especially helpful are JoAnne Wilken and Diane Bieber, who typed the manuscript, and Mr. N. Sanchez, who prepared most of the drawings. This research was funded by the Minerals Management Service of the U.S. Department of Interior. 


\section{REFERENCES}

[1] L. H. Larson, Ed., "Advances in Elasto-Plastic Fracture Mechanics," Applied Science Publishers, London, 1980.

[2] T. L. Anderson and H. I. McHenry, Natonal Bureau of Standards, NBSIR 83-1680, 1983.

[3] A. A. Griffith, Phi1. Trans. Roy. Soc. 221A, 1920, p. 163.

[4] G. R. Irwin and J. A. Kies, Welding J., 33, 1954, p. 193s.

[5] G. R. Irwin, Trans. ASTM, J. App1. Mech., 24, 1957, p. 361.

[6] H. Tada, P. C. Paris, and G. R. Irwin, The Stress Analys is of Cracks Handbook, Del Research Corp., Hellertown, Pennsylvania (1973).

[7] G. C. Sih, Handbook of Stress Intensity Factors for Researchers and Engineers, Institute of Fracture an Solid Mechanics, Lehigh University, Bethlehem, Pennsylvania (1973).

[8] G. R. Irwin, in: Proceedings Seventh Sagamore Ordinance Materials Research Conference, Syracuse University Press, Syracuse, New York (1960), p. IV-63.

[9] G. R. Irwin, Trans. ASME, J. Basic Eng. 82, 1960, p. 417.

[10] P. C. Paris and G. C. Sih, in: Fracture Toughness Testing and Its Applications, ASTM STP 381,1965, p. 30.

[11] A. A. Wells, Brit. Welding J., 12, 1965, p. 2.

[12] A. A. Wells, Brit. Welding J., 10 (11), 1963, p. 563.

[13] F. M. Burdekin and D. W. Stone, J. Strain Ana1., 1966. 
[14] D. S. Dugdale, J. Mech. Phys. Solids, 8 (2), 1960, p. 100.

[15] A. A. Wells, Congress of Applied Mechanics, Calgary, 1971, p. 57.

[16] F. M. Burdekin and M. G. Dawes, Proc. Mech. Eng. Conf., London, May 1971.

[17] M. G. Dawes, Advances in Elasto-Plastic Fracture Mechanics, edited by E. H. Larsson, Applied Science Publishers, London, 1980, p. 279.

[18] British Standard 5762: Methods for Crack Opening Displacement (COD) Testing, 1979, The British Standards Institution, London.

[19] J. R. Rice, J. Appl. Mech., 35 (2), 1968, p. 379.

[20] J. A. Begley and J. D. Landes, ASTM STP 514, 1972, p. 1.

[21] D. J. Hayes, Ph.D. Thesis, University of London, 1970.

[22] J. G. Blavel, Advances in Elasto-Plastic Fracture Mechanics, edited by E. H. Larsson, Applied Science Publishers, London, 1980, p. 65 .

[23] M. G. Dawes, The Welding Institute Research Bulletin, 13, 1982, p. 50 .

[24] J. F. Knott, Fracture 1977, vol. 1, ICF4, Water100, Canada, June, 1977 , p. 61.

[25] D. A. Curry and J. F. Knott, Metal Science, 10, 1976, p. 1.

[26] D. A. Curry, Nature, 276, 1978, p. 50.

[27] N. S. Stoloff, Fracture, An Advanced Treatise, vol. 6, edited by H. Lebowitz, Academic Press, New York, 1969, p. 2.

[28] J. F. Knott and A. H. Cottrel1, JISI, March, 1963, p. 249. 
[29] D. A. Curry and J. F. Knott, Metal Science, 13, 1979, p. 341.

[30] C. J. McMahan, Jr. and M. Cohen, Acta Met., 13, 1965, p. 591.

[31] J. F. Knott, JISI, 204, 1966, p. 104.

[32] T. R. Wilshaw, C. A. Rau, and A. S. Tetelman, Eng. Frac. Mech., 1, 1968 , p. 191.

[33] J. R. Griffiths, and D. R. J. Owen, J. Mech. Phys. Solids, 19, 1979 , p. 419.

[34] A. H. Cottre11, Trans. AIMME, 212, 1958, p. 192.

[35] E. Smith, Proceedings of the Conference on "The Physical Basis of Yield and Fracture," Inst. of Physics and Phys Soc., 1966, p. 36.

[36] R. E. Dolby and J. F. Knott, JISI, 210, 1972, p. 857.

[37] T. C. Lindley, G. Gates, and C. E. Richards, Acta Met., 18, 1970, p. 1127 .

[38] R. 0. Ritchie, J. F. Knott, and J. R. Rice, J. Mech. Phys. Solids, 21,1973, p. 395 .

[39] D. A. Curry, Metal Science, 14, 1980, p. 78.

[40] A. G. Evans and J. W. Hutchinson, to be published.

[41] A. R. Rosenfield and D. K. Shetty, Eng. Fract. Mech, 17, 1983, p. 461 .

[42] A. R. Rosenfield, D. K. Shetty, and A. J. Skidmore, Presented at the ASTM Symposium on User's Experimence with Elastic-Plastic Fracture Mechanics, Louisville, KY, April 1983.

[43] J. Q. Clayton and J. F. Knott, Metal Science, 10, 1976, p. 63.

[44] D. A. Curry and P. L. Pratt, Mat. Science Eng. 37, 1979, p. 223. 
[45] C. D. Beachem and G. R. Yoder, Met. Trans. 4, 1973, p. 1145.

[46] M. G. Dawes, Presented at the ASTM E-24-08 Committee meeting, Bal Harbor, FL, November 1980

[47] M. G. Dawes, ASTM STP 668, 1979, p. 307.

[48] A. B. Stuber, Ph.D. Thesis, University of Kansas, 1980.

[49] J. D. Landes, Int. J. Fract., 17, 1981, p. R47.

[50] A. Penelon, M. N. Bassim, and J. M. Dorlot, ASTM STP 677, 1979, p. 449 .

[51] I. Milne, Mat. Sci. Eng., 30, 1977, p. 243.

[52] G. G. Chell and G. M. Spink, Eng. Frac. Mech., 9, 1977, p. 101.

[53] I. Milne and P. J. Worthington, Mat. Sci. Eng., 26, 1976, p. 185.

[54] H. G. Pisarski, Int. J. Fract., 17, 1981, p. 427.

[55] H. W. Liv, W.-L. Hu, and A. S. Kuo, Presented at the ASTM Second International Symposium on Elastic-Plastic Fracture Mechanics, Philadelphia, PA, October 1981.

[56] P.M.S.T. de Castro, J. Spurrier, and P. Hancock, Int. J. Fract., $17,1981, \mathrm{p} 83$.

[57] G. G. Chell and A. Davidson, Mat. Sci. Eng., 24, 1976, p. 45.

[58] G. G. Chell and R. S. Gates, Int. J. Fract., 14 (2), 1978, p. 223.

[59] P. M. S. T. deCastro, J. Spurrier, and P. Hancock, ASTM STP 677, 1979, p. 486.

[60] J. D. Landes and D. H. Shaffer, ASTM STP 632, 1980, p. 368.

[61] F. A. McClintock and F. Zaverl Jr., Int. J. Fract., 15, 1979, p 107. 
[62] R. A. Hunt and L. M. McCartney, int. J. Fract., 15, 1979, p. 365.

[63] P. G. Tracy, T. P. Rich, R. Bonser, and L. R. Tramontozzi, Int. J. Fract., 18, 1982, p. 253.

[64] J. M. Barsom, Eng. Fract. Mech., 7, 1975, p. 605.

[65] B. Marandet and G. Sanz, A.STM STP 631, 1977, p. 72.

[66] J. M. Barsom and S. T. Rolfe, ASTM STP 466, 1970, p 181.

[67] R. H. Saitors and H. T. Corten, ASTM STP 514, 1972, p. 164.

[68] J. F. Sovak, J. Test. Evàl., 10, 1982, p. 102.

[69] M. G. Dawes, E. M. Remzi, and H. H. Tio, helding Institute Report $217 / 1983$, June 1983 .

[70] h. heibuli, J. Appl. Mech., 18, 1953.

[11] M. Saka, T. Shoji, H. Takahashi, and H. Abe, J. Mech. Phys. Solids, 30, 1982, p. 209.

[72] C. F. Shih and M. D. Geman, Int. J. Fract., 19, 1981, p. 27.

[73] L. P. Harrop, Int. J. Fract., 16, 1980, P. R199.

[74j S. H. Malik, L. S. Fu, Int. J. Fract., 18, 1982, p. 45.

[75] Y. Imaiand T. Matake, Eng. Fract. Mech., 16, i982, p. 659.

[76] M. Sakata, S. Aoki, K. Kishimoto, M. Kanzawa, and H. Ogure, Presented at the ASTM Second International Symposium on ElasticPlastic Fracture Mechanics, Philadelphia, PA, October 1981.

[77] M. G. Dawes, The helding Institute Research Bulletin, 7, 1976, p. 185 .

[78] I.-H. Lin, T. L. Anderson, R. dehit and M. G. Dawes, Int. J. Fract., 20, 1982, p. R3. 
[79] J.D.G. Sumpter and C. E. Turner, ASTM STP 601, 1976, P. 3.

[80] J. R. Rice, P. C. Paris and J. G. Merkle, ASTM STP 536, 1973, p. 231.

[81] G. E. Dieter, Mechanical Metallurgy, McGraw Hi11, New York, 1976.

[82] D. M. Tracey, Trans. ASME, 98, 1976, p. 146.

[83] T. Shoji, H. Takahashi and M. Suzuki, Metal Science, 12, 1978, p. 579 .

[84] J. F. Knott, Metal Seicne, 14, 1980, p. 327.

[85] 0. A. Onyewuenyi and J. P. Hirth, Metal1. Trans., 13, 1982, p. 2209.

[86] R. 0. Ritchie, R. F. Smith, and J. F. Knott, Metal Science, 9, 1975, p. 485.

[87] 0. L. Towers, Welding Institute Report 136/1981, March 1981. 


\section{APPENDIX I:}

ADVANCES IN FRACTURE TOUGHNESS TEST METHODS

New developments and refinements in fracture toughness test methods which resulted from this investigation are described below. These results, although important, were not included in the main body of this report because they are not part of the central theme of this dissertation.

\subsection{The Rotational Factor}

The rotational factor, $r_{p}$, is defined as the distance from the hinge point (in an SENB specimen) divided by the ligament length. The rotational factor was previously defined in equation (31) where $r_{p}$ is expressed as a function of specimen dimensions and the plastic components of mouth-opening $\left(V_{p}\right)$ and load-line $\left(q_{p}\right)$ displacements [57]:

$$
r_{p}=\frac{1}{w-a} \frac{v_{p} W}{q_{p}}\left[\left(1 \frac{q_{p}}{16 W}\right)-(a+z)\right]
$$

The above equation was derived assuming the SENB specimen deforms in pure bending about a hinge point; the specimen halves are assumed to remain rigid.

The rotational factor was measured for five geometries of ABS EH36 steel SENB specimens at various temperatures. Figure 71 shows that $r_{p}$ 


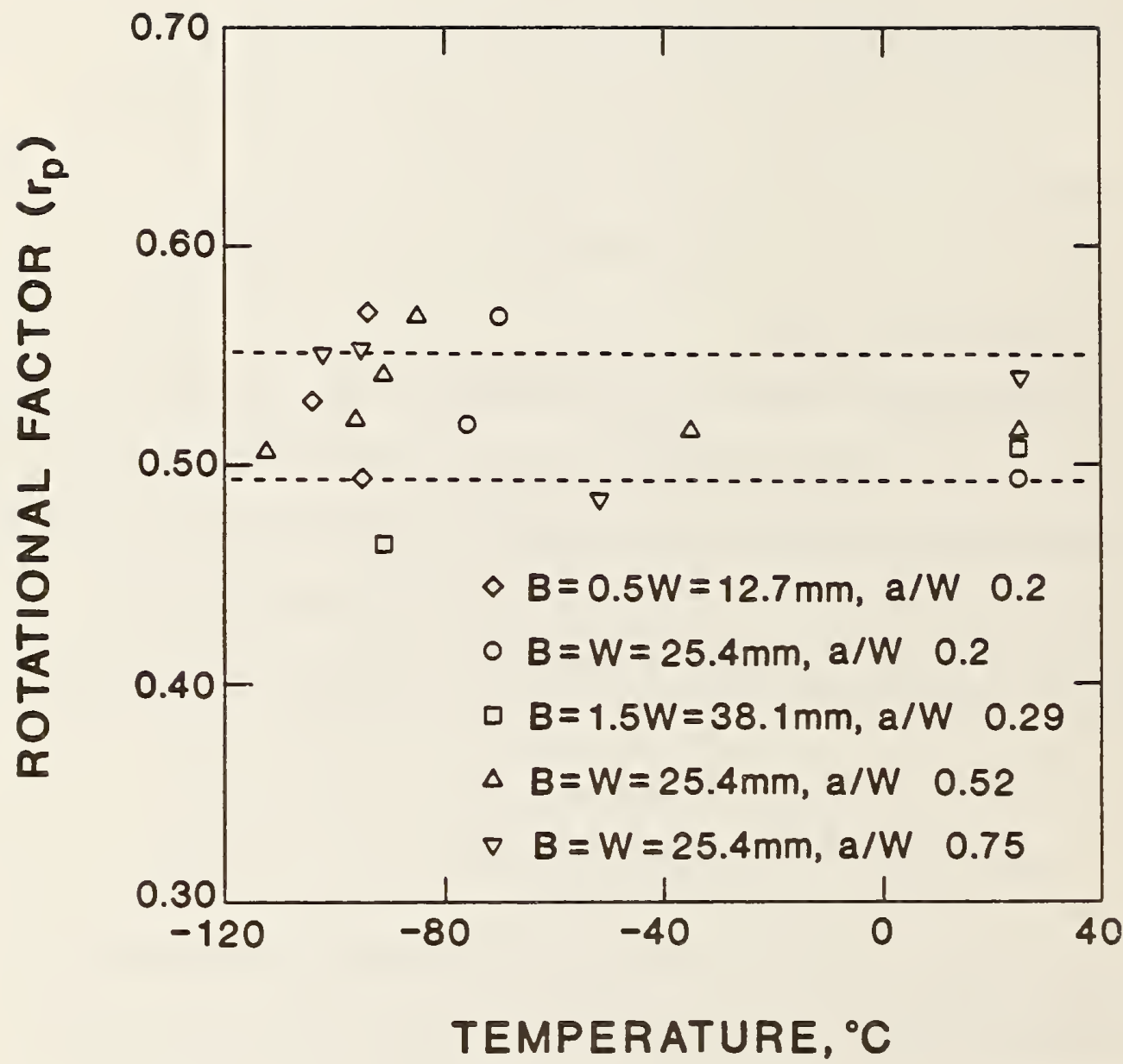

Figure 71. Plastic rotational factor as a function of SENB specimen geometry and tempterature for ABS grade EH36 steel. 
is insensitive to geometry and test temperature. The mean value of the data in Figure $6 a$ is 0.522 ; the standard ceviation is 0.030 .

Equation (64) can be used to determine the sensitivity of CTOD measurements to variations in $r_{p}[57]$ :

$$
\frac{d(\partial p / V p)}{d r_{p}}=\frac{(a+z)(W-a)}{\left[r_{p}(W-a)+a+z\right]^{2}}
$$

Using $r_{p}=0.5, W=25.4 \mathrm{~mm}, a=12.7 \mathrm{~mm}$ and $z=1.3 \mathrm{~mm}$, the above derivative is equal to 0.43 . A variation in $r_{p}$ of 0.030 ( 1 standard deviation) corresponds to an error in the op measurement of approximately 4 percent. Therefore, CTOD calculations are not highly sensitive to $r_{p}$ variations.

\subsection{Detecting the Onset of Tearing}

During a fracture test with an SENB specimen the load-7ine displacement (q) was plotted continuously against the crack mouth-opening displacement $(V)$. After net-section yield the a versus $V$ plot is a nearly straight line. However, as the crack grows this 1 ine gradually decreases in slope because the center of rotation moves during crack growth. Figure 72 shows a plot of the first derivative of $q$ with respect to $V$. The behavior at the far left of this plot represents the transition from elastic to plastic deformation. During plastic deformation the first derivative remains relatively constant and then 


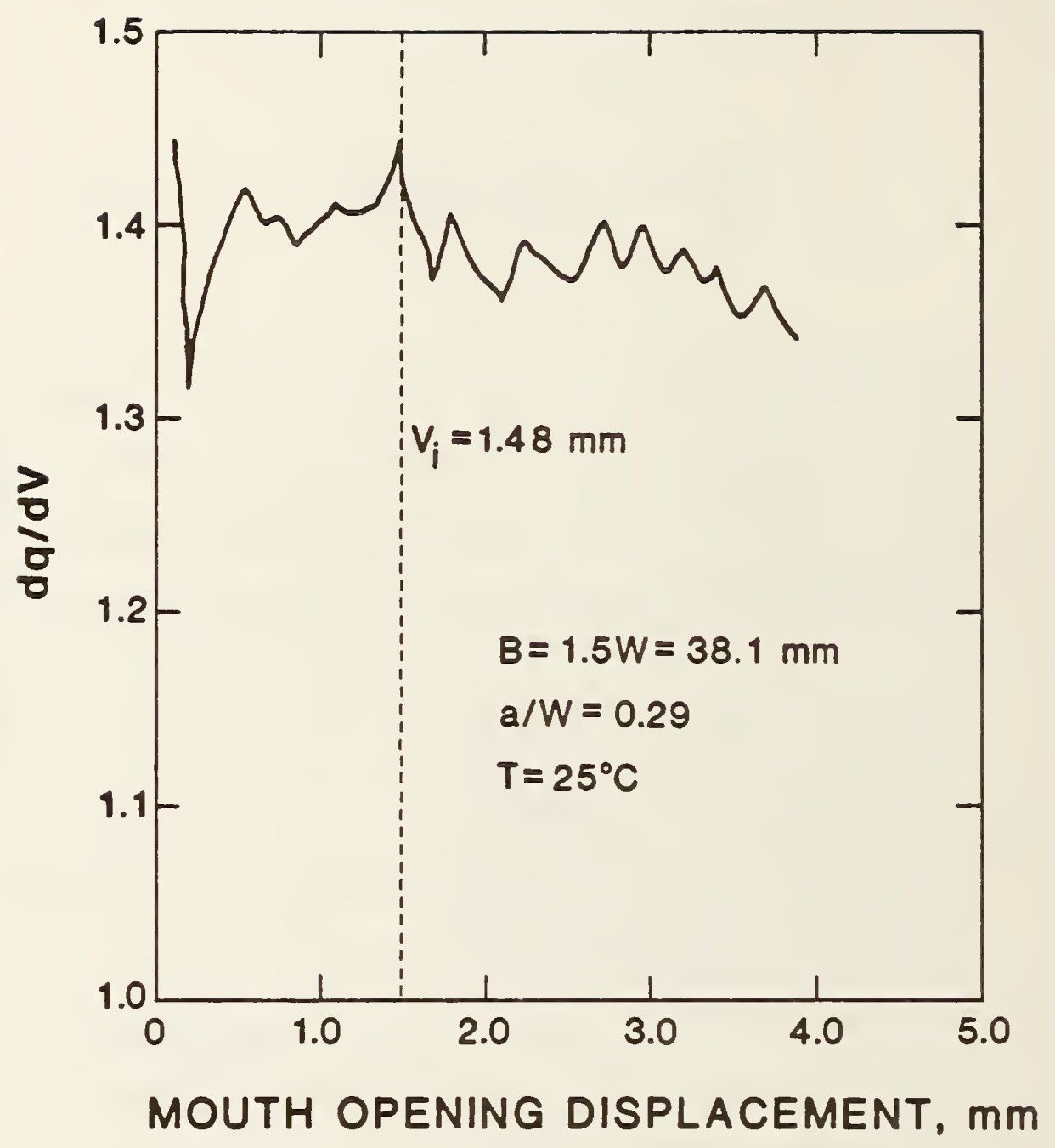

Figure 72. Derivative of $q$ with respect to $V$. A sharp drop in the first derivative generally indicates tearing. 
increases sharply. At $V=1.48 \mathrm{~mm}$ there is a sharp drop in the first derivative. This is taken as the point of incipient tearing. This behavior is characteristic of fracture tests where tearing was observed. Observation of fracture surfaces of specimens that fractured prior to and immediately following the onset of tearing and the corresponding derivative plots indicate that the sharp drop in the first derivative coincides with incipient tearing. At higher displacements the curve has a zig-zag shape. This is believed to be associated with successive increments of crack growth.

Figure 72 is typical of the first derivative plots from fracture tests in which tearing was observed. The spike in the curve, which was taken as the point of incipient tearing, is slighly more pronounced than the other spikes. Often the initiation point is $i 11$ defined because of the uncertainty of which spike corresponds to the onset of stable crack growth.

\subsection{Estimation of $J$ from CMOD}

For deep-notched SENB specimens the J-integral is usually estimated from equation (32):

$$
J=\frac{2 U}{B(W-a)}
$$


where $U$ is the total area under the load versus load-line displacement curve. The value of $\mathrm{J}$ can also be estimated from the load versus mouth opening displacement curve using equation (33):

$$
J=\frac{k^{2}}{E^{\prime}}+\frac{2 U_{p}^{v}}{B(W-a)}\left(\frac{W}{r_{p}(W-a)+a+z}\right)
$$

where $U_{P}^{V}$ is the plastic component of the area under the $P / V$ curve. Equation (33) is based on the Sumpter and Turner [79] equation, al though they approximated the area by

$$
U_{p}^{V} \cong P_{L} V_{p}
$$

where $P_{L}$ is the limit load:

The J-integral has been calculated for a number of tests using equations (32) and (33) and the agreement is very good. Figure 73 shows a comparison of estimates from the two equations. The data represent a single specimen at various displacements. The difference between the two $\mathrm{J}$ estimates for this particular specimen range from $0.5 \%$ to $1.5 \%$.

The value of $r_{p}$ used in equation (33) to generate Figure 73 was measured from the same specimen using equation (31). The excellent agreement between equations (32) and (33) as shown in Figure 73 is evidence that the simple hinge mechanism is an adequate model to describe the plastic deformation of an SENB specimen. Figure $7 \dot{3}$ also 


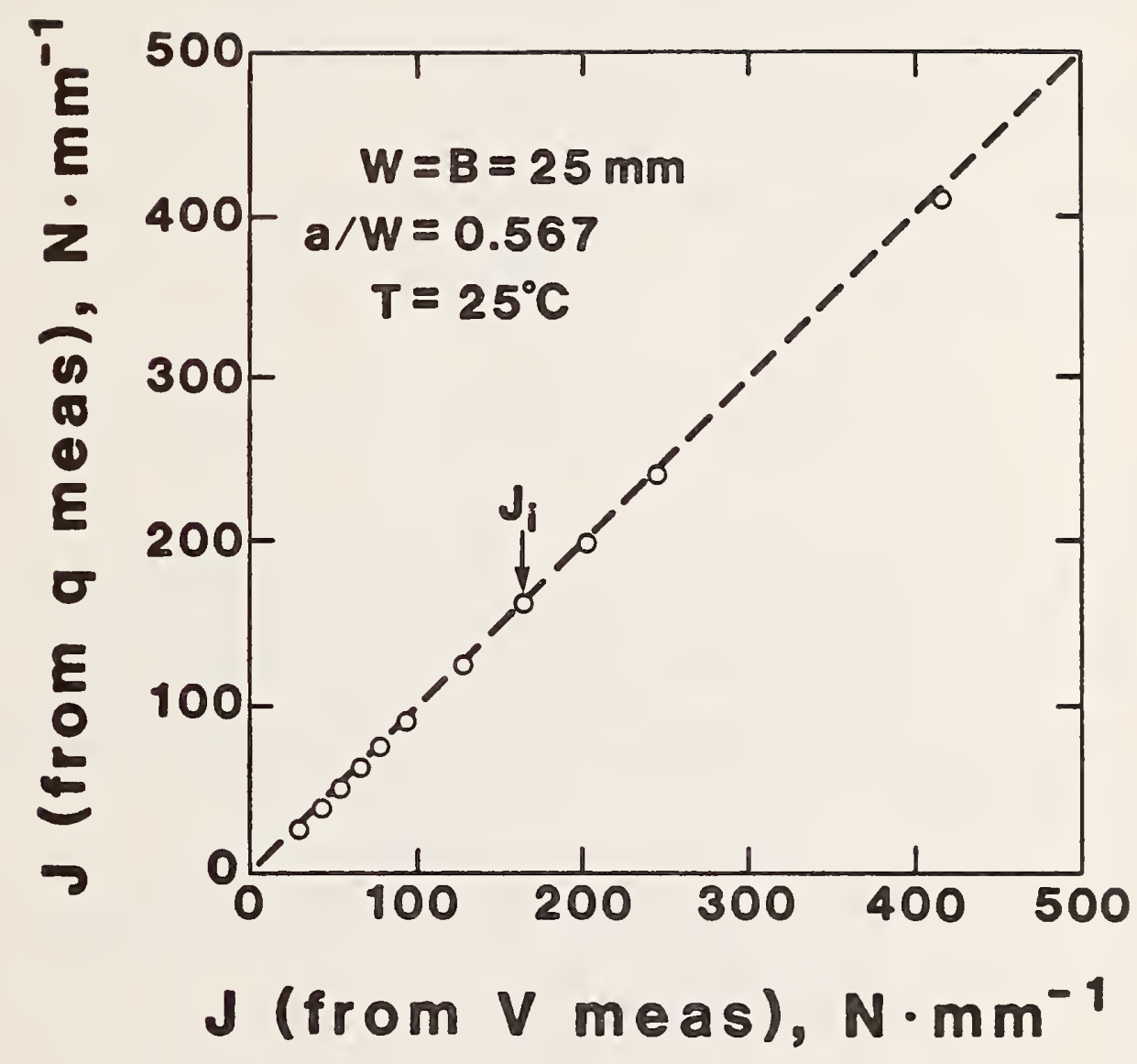

Figure 73. Comparison of $\mathrm{J}$ estimates from equations (32) and (33). 
shows that the J-integral can be accurately measured from the mouth-opening displacement if $r_{p}$ is known. Thus, it is possible to measure both $J$ and CTOD from an SENB specimen with a single clip gage.

\subsection{Relationships Between $\mathrm{J}$ and CTOD}

Under conditions of small-scale yielding the relationship between $\mathrm{J}$ and CTOD can be estimated by:

$$
J=m \sigma_{0} \delta
$$

where $m=1$ for $p l a n e$ stress and $m=2$ for plane strain [47]. The value of $\mathrm{m}$ for large-scale yielding should 1 ie between 1.0 and 2.0 .

The plastic term in equation (33) is similar to the equation for the plastic CTOD:

$$
\delta_{p}=\frac{r_{p}(W-a) v_{p}}{r_{p}(W-a)+a+z}
$$

From equations (33) and (66) one can obtain a simple equation for the ratio of the plastic $J$ to the plastic CTOD

$$
\frac{J_{p}}{\delta_{p}}=\frac{2 U_{p}^{V_{W}}}{V_{p} B r_{p}(W-a)^{2}}
$$


Equation (67) has been incorporated into a computer program which plots $J p / \delta p$ as a function of mouth-opening displacement. A typical plot is shown in Figure 74 . The ratio $\mathrm{Jp} / \delta p$ increases with displacement because of work hardening. The work hardening can be accounted for by replacing the yield stress in equation (65) with a nominal flow stress:

$$
J_{p}=m_{p} \sigma_{f l o w} \delta_{p}
$$

The nominal flow stress can be estimated by

$$
\sigma_{\text {flow }}=\frac{\bar{P}}{P_{y}} \sigma_{0}
$$

where $\bar{P}$ is the average load defined as

$$
\bar{P}=\frac{U_{p}^{v}}{V p}
$$

and $P_{y}$ is the load at yield defined previously in equation (35):

$$
P_{y}=\frac{L \sigma_{0}(W-a)^{2} B}{4 W}
$$

Combining equations (35) and (67)-(70) yields the following relationship for $m_{p}$.

$$
m_{p}=\frac{L}{2 r_{p}}
$$

For the ABS grade EH36 steel, $r_{p} \cong 0.5$; therefore, $m_{p} \cong L$ (the notch constraint factor). Thus, the relationship between plastic components 


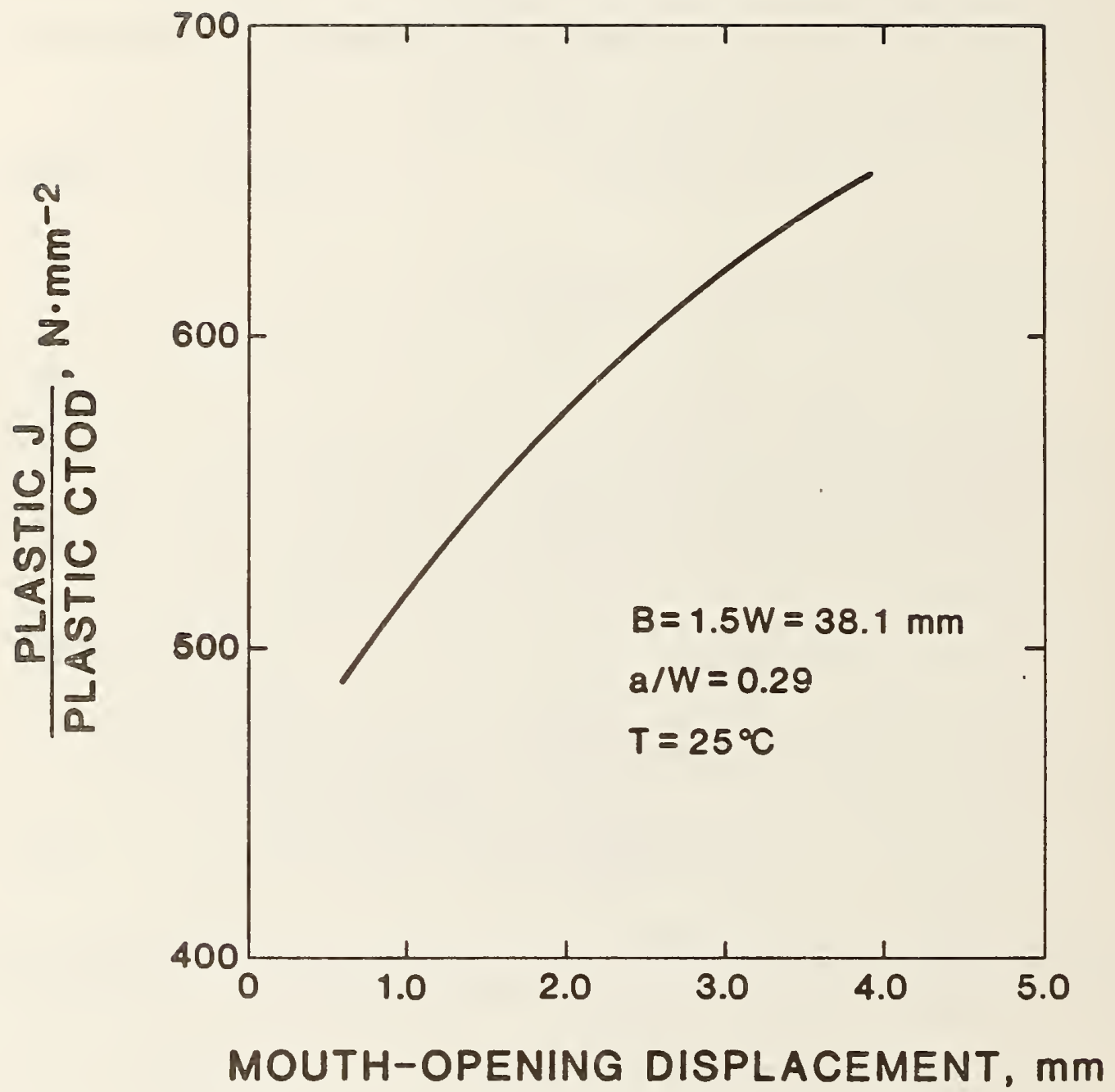

Figure 74 . The ratio of the plastic components of $J$ and CTOD as a function of displacement. 
of $J$ and CTOD (eq. 69) depends on the geometrical constraint of the specimen and the flow properties of the materiat.

\subsection{The Eta Factor}

A more general form of equation (32) is:

$$
J=\frac{n U}{B(W-a)}
$$

where $n \cong 2$ for deep notched SENB specimens. The above equation can be divided into elastic and plastic components [79]:

$$
J=\frac{n_{e} e_{e}}{B(W-a)}+\frac{n_{p} U_{p}}{B(W-a)}=\frac{K}{E^{\prime}}+\frac{n_{p} U_{p}}{B(W-a)}
$$

The elastic eta factor, $n_{e}$, can be derived from the elastic compliance and stress intensity coefficient. The solid line in Figure 75 is a plot of $n_{e}$ versus notch depth [87].

Since $J$ is defined as the negative of the spatial derivative of work, the plastic eta factor, $n_{p}$, and the over-all eta factor, $n_{0}$, can be computed as follows:

$$
J=\frac{n_{0} U}{B(W-a)}=-\frac{1}{B} \frac{d U}{d a}
$$




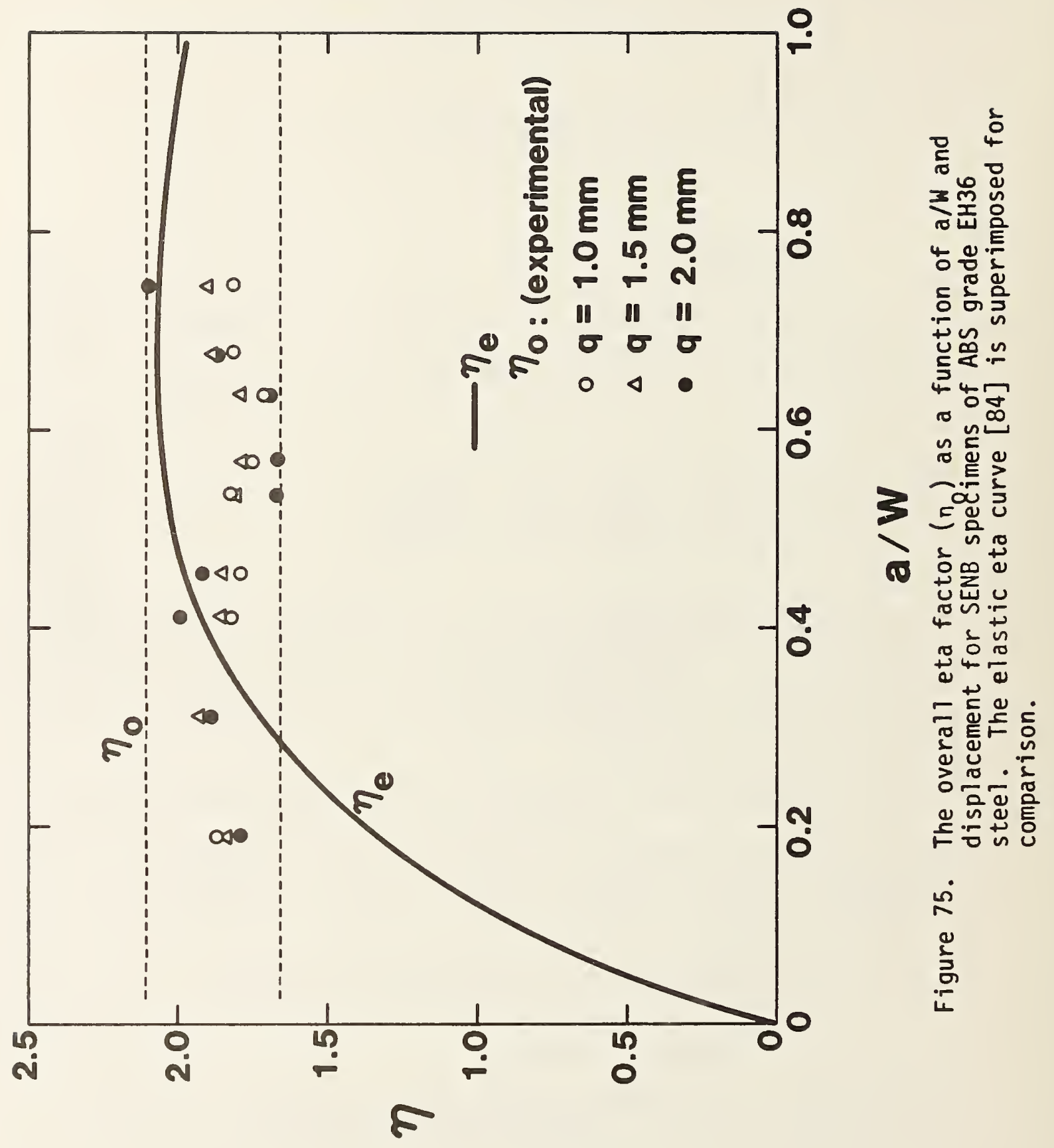


hence

$$
n_{0}=-\frac{(W-a)}{U} \frac{d U}{d a}
$$

A series of SENB specimens with notch depths ranging from $a / W=0.19$ to 0.75 were tested at room temperature. The area under the load versus loadline displacement curve, $U$, was measured for each specimen at displacements of $1.0,1.5$, and $2.0 \mathrm{~mm}$. These values of $U$ were then plotted versus crack length and dU/da was measured for various crack lengths by drawing tangents to the $U$ versus a curves and computing the slopes. The over-all eta factor, $n_{0}$, was then computed as a function of crack length. The results are plotted in Figure 75. According to Figure 75, $n_{0}$ is relatively independent of crack length down to $\mathrm{a} / \mathrm{W}=0.19$. It therefore seems reasonable to use a value of $n=1.8-2.0$ for all $\mathrm{J}$ measurements from SENB specimens with $a / W \geq 0.2$.

\subsection{Crack-Tip Replication}

The crack tips of three ABS EH36 steel fracture specimens were replicated with a plastic impression material. These replicas were made at various CTOD values by pouring the impression material into the crack mouth. When the impression material hardened the replica was removed from the crack and later sectioned. The crack-tip opening displacement 
was measured directly by placing each sectioned replica under a microscope equipped with a stage micrometer and a measuring eyepiece.

Measured and computed values of CTOD are compared in Table 11. The agreement is reasonably good al though there are discrepancies at high CTOD values. The computed CTOD is defined at the original crack tip; when significant blunting and tearing has occurred it is difficult to determine the location of the original crack tip. This might account for the differences between computed and measured high CTOD values. 
8. APPENDIX II:

TABULATED DATA 


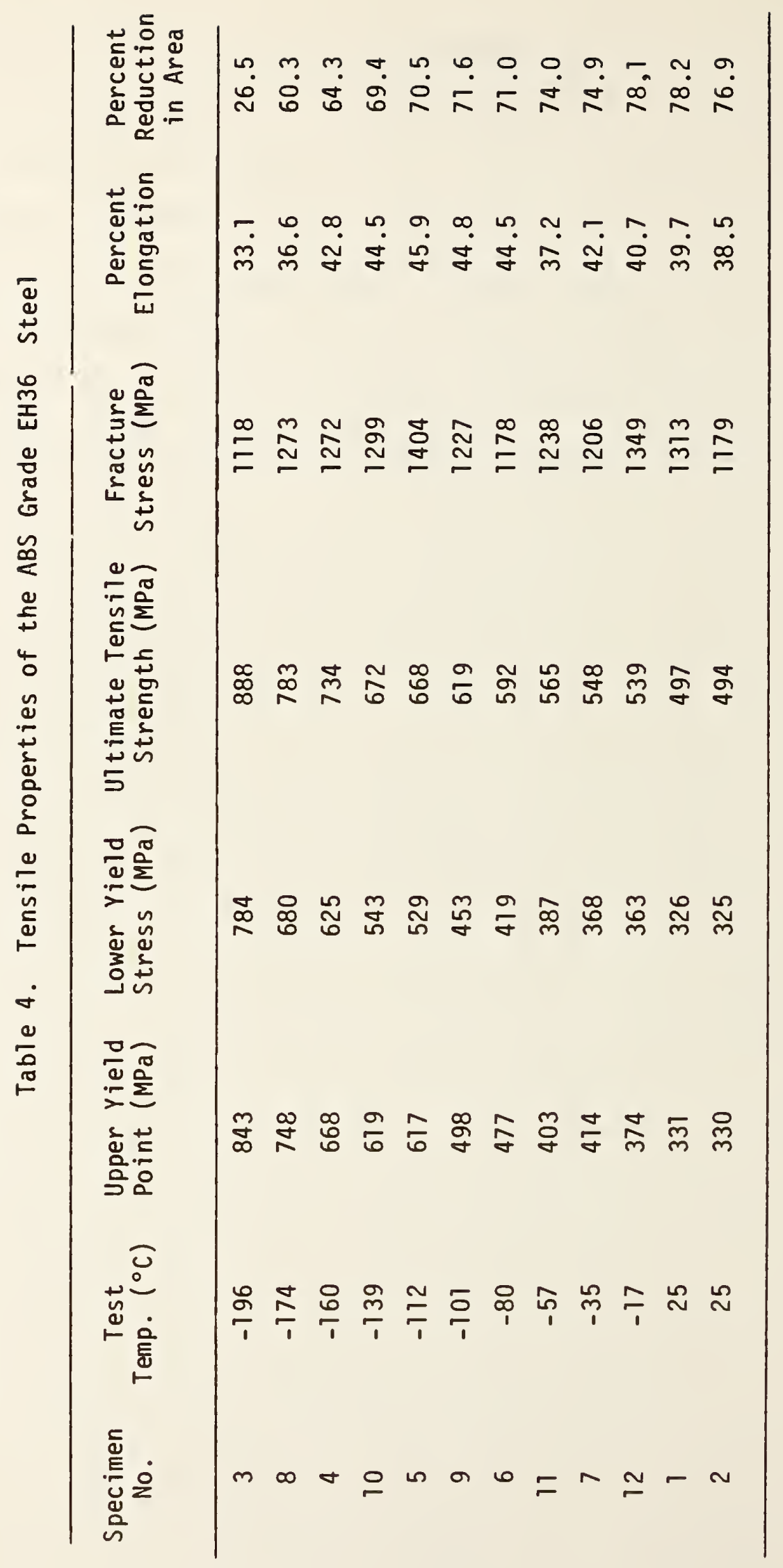




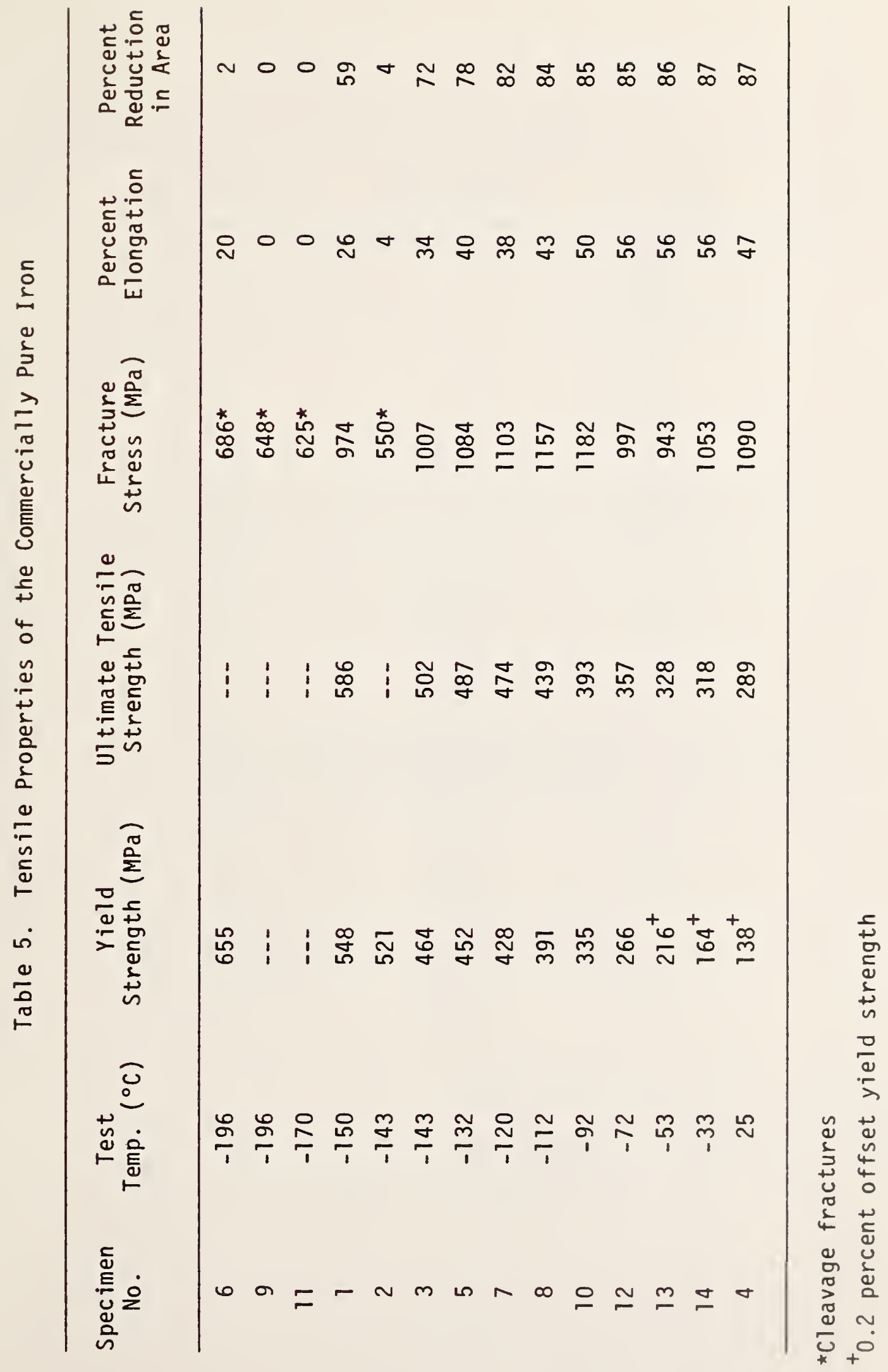


Table 6. Charpy V-Notch Impact Data for ABS Grade EH36 Steel

\begin{tabular}{ccccc}
\hline Specimen No. & Temp $\left({ }^{\circ} \mathrm{C}\right)$ & $\begin{array}{c}\text { Absorbed } \\
\text { Energy }(\mathrm{J})\end{array}$ & $\begin{array}{c}\text { Lateral } \\
\text { Expansion }(\mathrm{mm})\end{array}$ & $\begin{array}{c}\text { Percent } \\
\text { Shear }\end{array}$ \\
\hline 4 & -190 & 1 & 0.041 & 0 \\
5 & -125 & 4 & 0.055 & 0 \\
6 & -95 & 11 & 0.145 & 0 \\
14 & -93 & 106 & 1.565 & 10 \\
15 & -85 & 22 & 0.345 & 0 \\
13 & -82 & $323^{*}$ & 1.800 & 100 \\
11 & -80 & 9 & 0.170 & 0 \\
17 & -76 & 20 & 0.342 & 0 \\
16 & -75 & 225 & 2.395 & 55 \\
19 & -75 & $355^{*}$ & 1.483 & 100 \\
20 & -75 & 41 & 0.186 & 0 \\
10 & -73 & $315^{*}$ & 2.293 & 100 \\
12 & -73 & $315^{*}$ & 1.843 & 100 \\
18 & -71 & 233 & 2.264 & 58 \\
7 & -70 & 57 & 0.859 & 3 \\
9 & -63 & $334^{*}$ & 1.890 & 100 \\
8 & -49 & $323^{*}$ & 1.630 & 100 \\
3 & 0 & $355^{*}$ & 2.285 & 100 \\
1 & 25 & $336^{*}$ & 2.500 & 100 \\
2 & 25 & $355^{*}$ & 2.220 & 100
\end{tabular}

*Exceeds $80 \%$ of the full-scale energy

${ }^{+}$Specimen did not fracture completely 


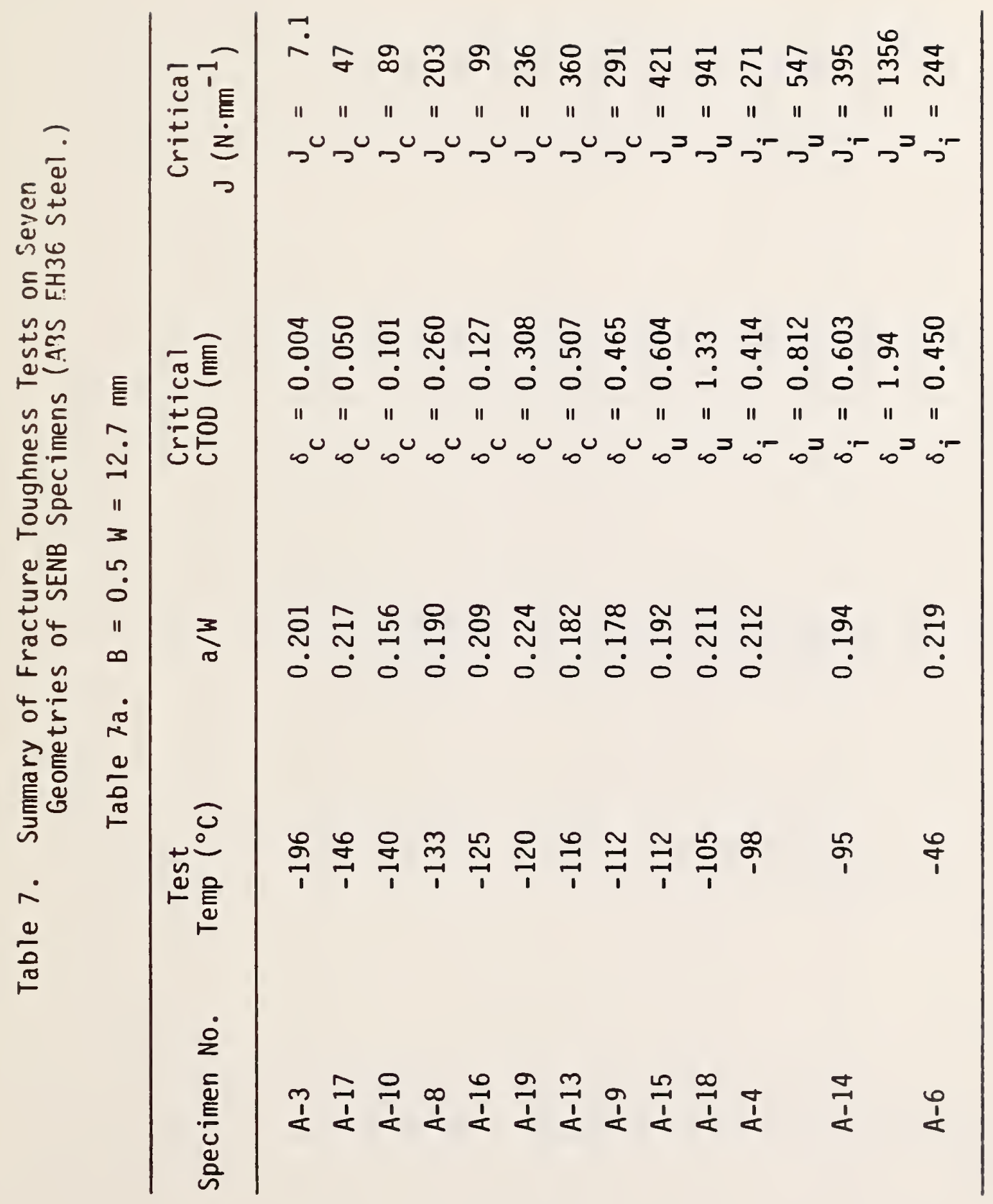




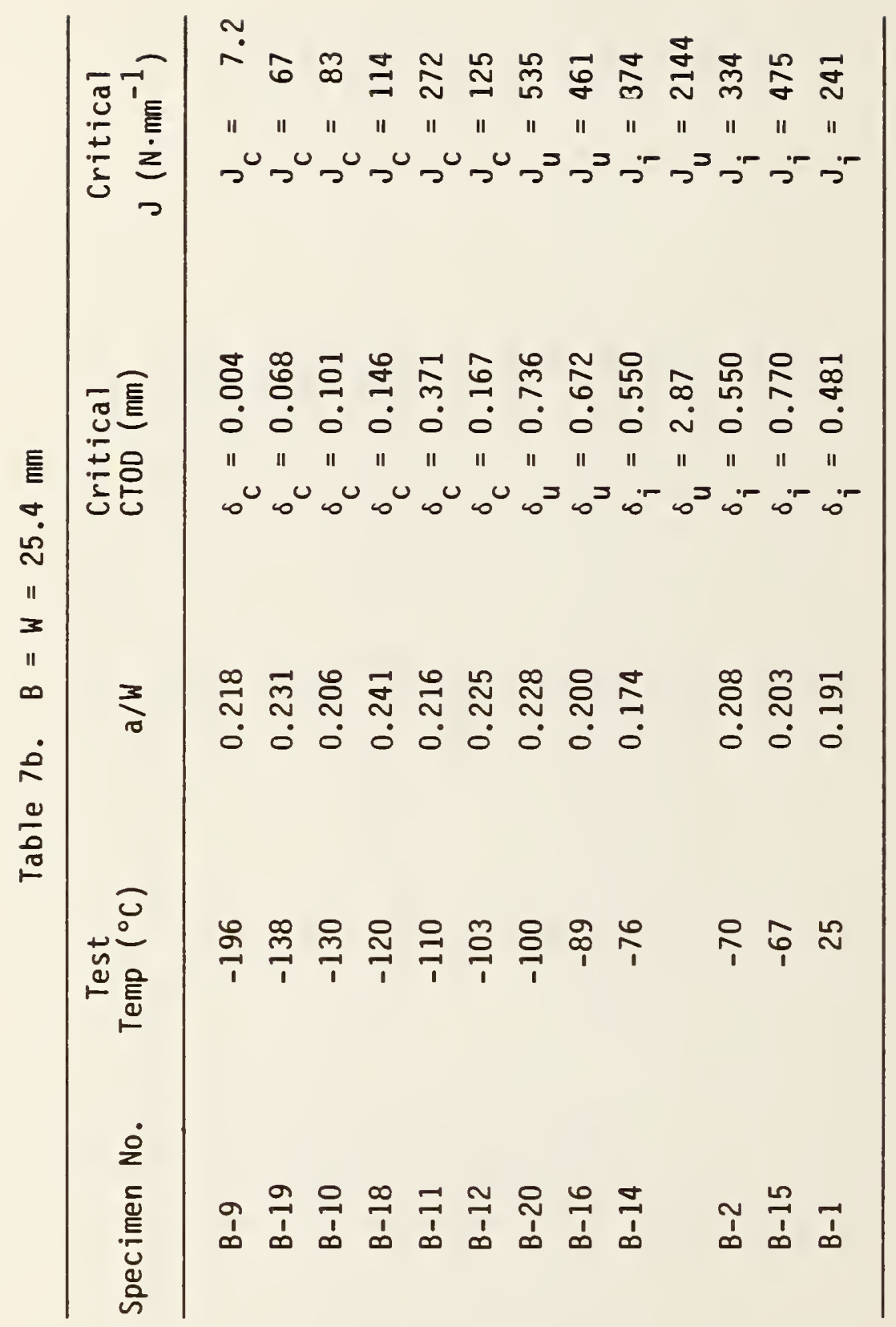




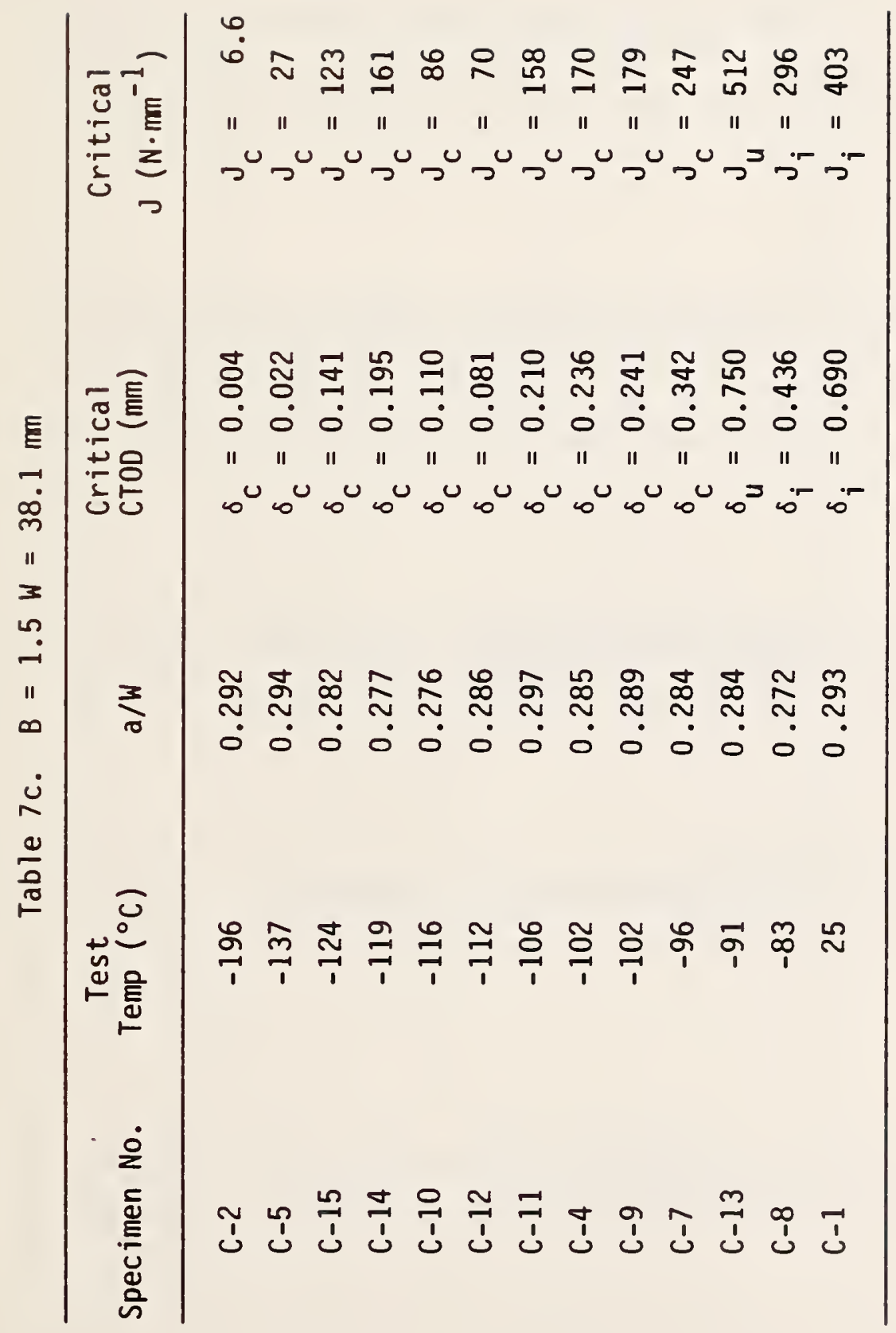




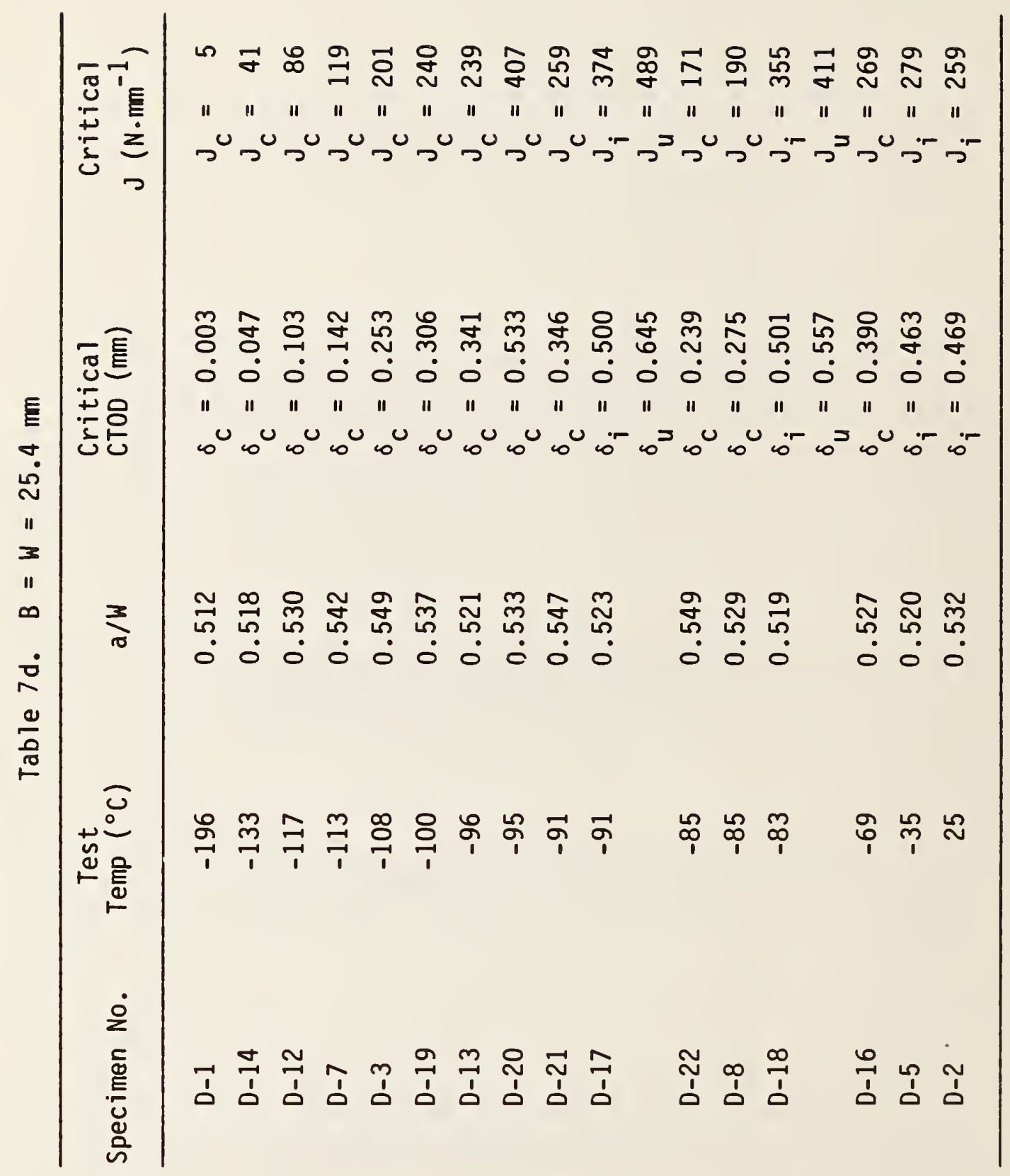




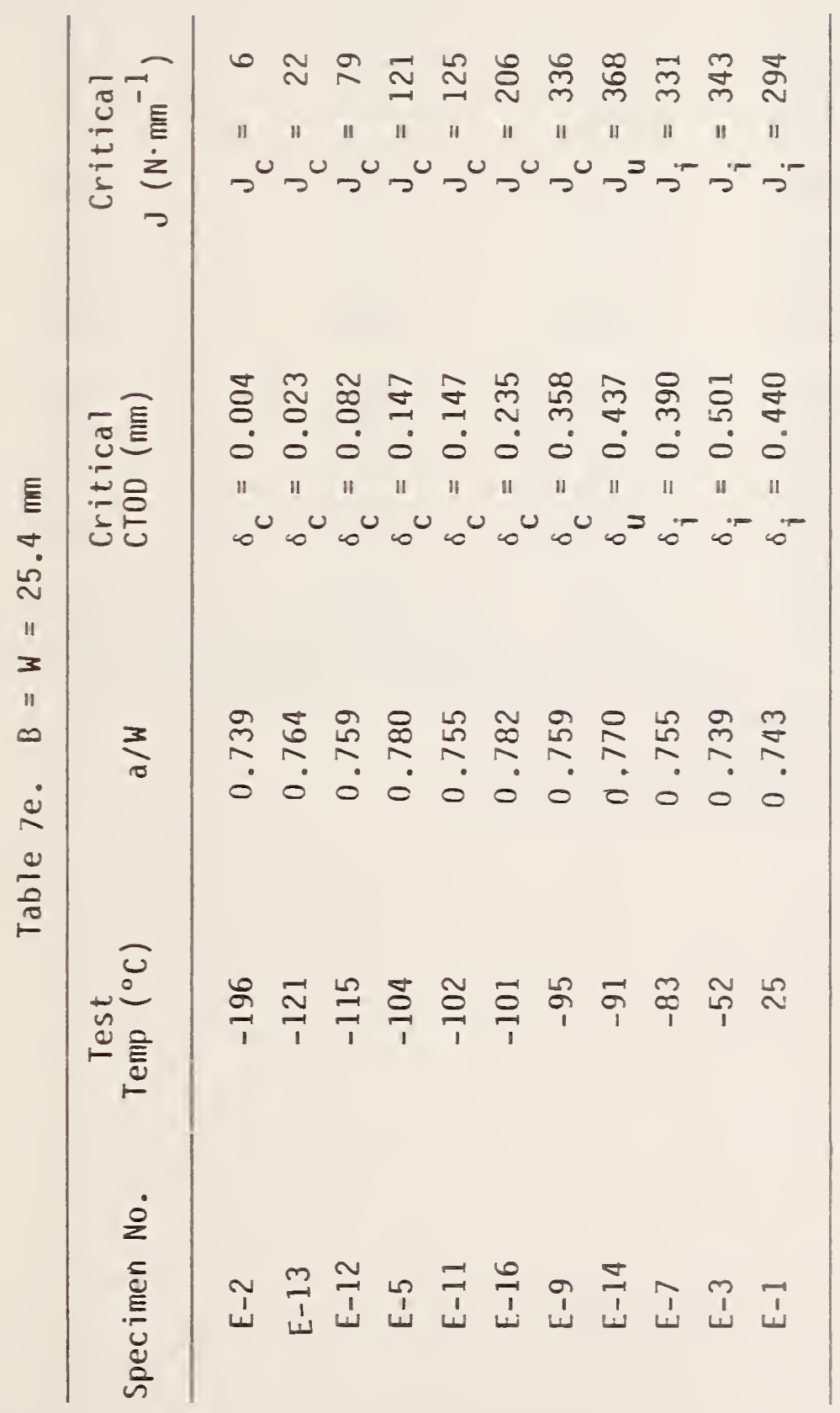


Table 7f. $B=3 W=76.2 \mathrm{~mm}$

\begin{tabular}{|c|c|c|c|c|}
\hline Specimen No. & $\begin{array}{c}\text { Test } \\
\text { Temp. }\left({ }^{\circ} \mathrm{C}\right)\end{array}$ & $a / w$ & $\begin{array}{l}\text { Critical } \\
\text { CTOD (mm) }\end{array}$ & $\begin{array}{l}\text { Critical } \\
J\left(N \cdot \mathrm{mm}^{-1}\right)\end{array}$ \\
\hline$F-2$ & -110 & 0.506 & 0.108 & 86 \\
\hline$F-5$ & -105 & 0.519 & 0.335 & 260 \\
\hline$F-1$ & -101 & 0.542 & 0.154 & 118 \\
\hline$F-8$ & -97 & 0.535 & 0.423 & 320 \\
\hline$F-6$ & -95 & 0.502 & 0.200 & 120 \\
\hline$F-3$ & -93 & 0.522 & 0.120 & 88 \\
\hline$F-7$ & -86 & 0.507 & 0.364 & 250 \\
\hline$F-4$ & -85 & 0.520 & $0.800 *$ & 626 \\
\hline
\end{tabular}

All fractures were by cleavage

* Cleavage preceded by stable crack growth 
Table 7g. $B=1.60 \mathrm{~W}=25.4 \mathrm{~mm}$

\begin{tabular}{ccccc}
\hline Specimen No. & $\begin{array}{c}\text { Test } \\
\text { Temp. }\left({ }^{\circ} \mathrm{C}\right)\end{array}$ & $a / W$ & $\begin{array}{l}\text { Critica } \\
\text { CTOD (mm })\end{array}$ & $\begin{array}{l}\text { Critica } \\
\mathrm{J}(\mathrm{N} \cdot \mathrm{mm}-1\end{array}$ \\
\hline G-6 & -123 & 0.215 & 0.063 & 52 \\
G-5 & -116 & 0.232 & $0.572^{\star}$ & $433^{*}$ \\
G-7 & -114 & 0.206 & 0.386 & 285 \\
G-4 & -110 & 0.214 & 0.094 & $\mathrm{na}$ \\
G-8 & -105 & 0.258 & 0.380 & 277 \\
G-1 & -103 & 0.228 & 0.046 & 38 \\
G-3 & -95 & 0.250 & $0.646^{*}$ & 470 \\
G-2 & -87 & 0.215 & 0.437 & 290 \\
\hline
\end{tabular}

All fractures were by cleavage

* Cleavage preceded by stable crack growth 
Table 8. Summary of Fracture Toughness Tests on Three Geometries of SENT Specimens (ABS EH36 Steel) $W=25.4 \mathrm{~mm}$

\begin{tabular}{cccccc}
\hline Specimen No. & $\begin{array}{c}\text { Test } \\
\text { Temp. }\left({ }^{\circ} \mathrm{C}\right)\end{array}$ & a/W & B/W & $\begin{array}{c}\text { Critical } \\
\text { CTOD (mm) }\end{array}$ & $\begin{array}{c}\text { Critical } \\
\text { J(N.mm }\end{array}$ \\
\hline H-4 & -133 & 0.203 & 1 & 0.050 & $n a$ \\
H-1 & -123 & 0.246 & 1 & 0.184 & 94 \\
H-2 & -115 & 0.235 & 1 & 0.184 & 82 \\
H-3 & -106 & 0.217 & 1 & $0.503^{*}$ & 282 \\
H-5 & -89 & 0.203 & 1 & $0.630^{*}$ & 366 \\
I-4 & -120 & 0.527 & 1 & 0.208 & 234 \\
I-1 & -111 & 0.544 & 1 & 0.101 & 58 \\
I-6 & -110 & 0.532 & 1 & 0.370 & 266 \\
I-3 & -101 & 0.534 & 1 & $0.540^{*}$ & 444 \\
I-2 & -96 & 0.532 & 1 & 0.370 & 348 \\
I-5 & -84 & 0.533 & 1 & $0.666^{*}$ & 521 \\
L-2 & -120 & 0.540 & 3 & 0.240 & 153 \\
L-1 & -105 & 0.537 & 3 & 0.374 & 391 \\
L-4 & -100 & 0.522 & 3 & 0.418 & 240 \\
L-5 & -92 & 0.533 & 3 & 0.588 & 242 \\
L-3 & -83 & 0.521 & 3 & 0.706 & 551 \\
\hline
\end{tabular}

A11 fractures were by cleavage

* Cleavage preceded by stable crack growth 
Table 9. Summary of Fracture Toughness Tests on Two Geometries of Pure Iron SENB Specimens $W=25.4 \mathrm{~mm}$

\begin{tabular}{lccccc}
\hline Specimen No. & $\begin{array}{c}\text { Test } \\
\text { Temp. }\left({ }^{\circ} \mathrm{C}\right)\end{array}$ & $\mathrm{a} / \mathrm{W}$ & $\mathrm{B} / \mathrm{W}$ & $\begin{array}{l}\text { Critical } \\
\text { CTOD (mm) }\end{array}$ & $\begin{array}{c}\text { Critica] } \\
\text { J(N.mm }\end{array}$ \\
\hline J-4 & -93 & 0.223 & 1 & 0.010 & 6.6 \\
$J-5$ & -84 & 0.216 & 1 & 0.092 & 45 \\
J-6 & -79 & 0.229 & 1 & 0.217 & 115 \\
J-9 & -76 & 0.228 & 1 & 0.199 & 97 \\
J-3 & -76 & 0.200 & 1 & 0.438 & 209 \\
J-7 & -72 & 0.230 & 1 & 0.540 & 288 \\
J-8 & -69 & 0.201 & 1 & 0.185 & 85 \\
K-8 & -90 & 0.197 & 2 & 0.029 & 13 \\
K-7 & -84 & 0.187 & 2 & 0.046 & 20 \\
K-1 & -74 & 0.207 & 2 & 0.140 & 70 \\
K-9 & -69 & 0.195 & 2 & 0.426 & 209 \\
K-6 & -67 & 0.187 & 2 & 0.628 & 310 \\
K-2 & -63 & 0.205 & 2 & 0.159 & 68 \\
K-4 & -58 & 0.222 & 2 & 0.308 & 136 \\
& & & & &
\end{tabular}

A11 fractures were by cleavage 


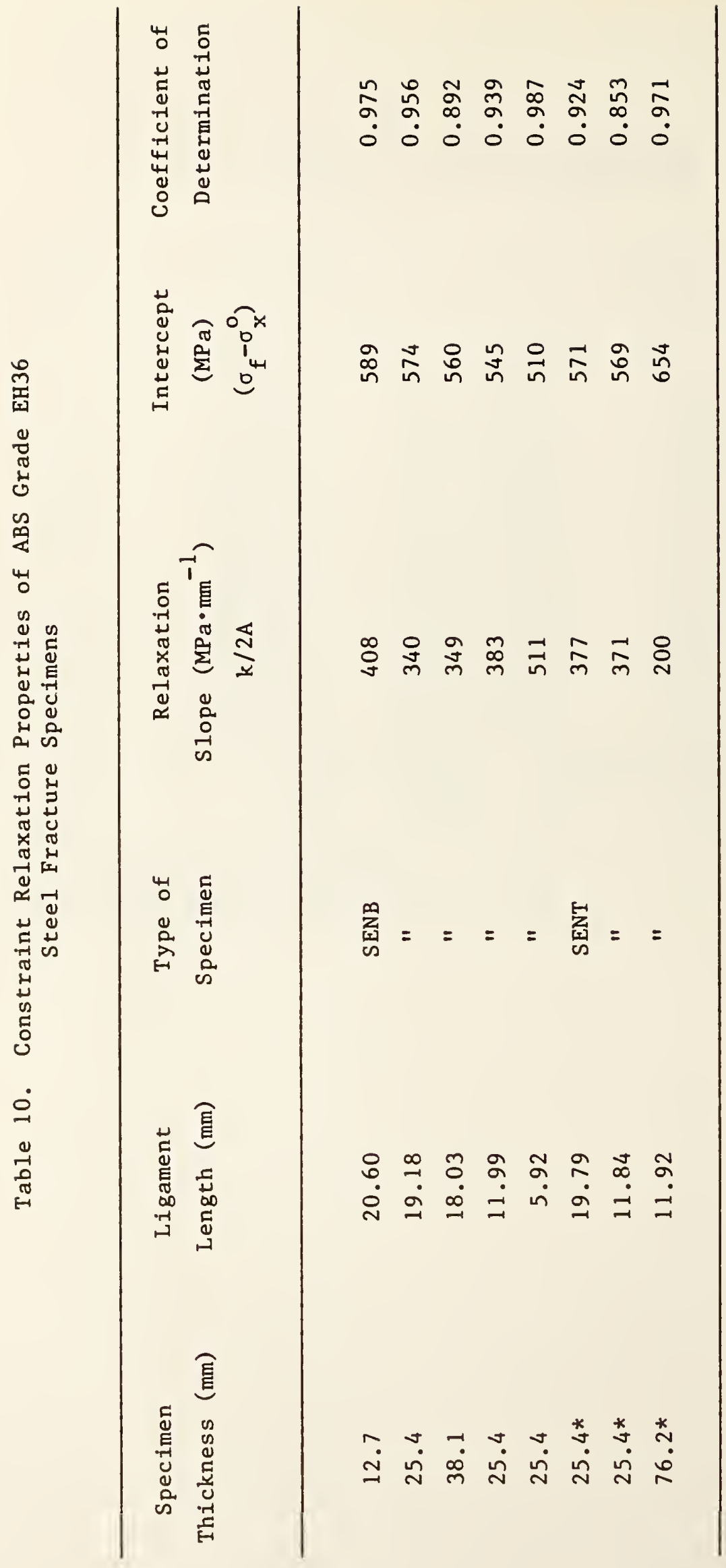


Table 11. Comparison of Computed CTOD Values with Crack-Tip Replica CTOD Measurements $\left(\mathrm{T}=25^{\circ} \mathrm{C}, \mathrm{B}=\mathrm{W}=25.4 \mathrm{~mm}\right)$

Table 11a. SENB Specimen $B-17, a / W=0.226$

\begin{tabular}{ccc}
\hline Replica No. & Measured & $\begin{array}{c}\text { CTOD (mm) } \\
\text { Computed From Eqn. (30) }\end{array}$ \\
\hline & & 0.297 \\
3 & 0.250 & 0.547 \\
& 0.540 & 1.041 \\
\hline
\end{tabular}

Table 1lb. SENB Specimen B-17, $a / W=0.550$

\begin{tabular}{ccc}
\hline Replica No. & Measured & $\begin{array}{c}\text { CTOD (mm) } \\
\text { Computed from Eqn. (30) }\end{array}$ \\
\hline 4 & 0.230 & 0.238 \\
5 & 0.500 & 0.490 \\
6 & 0.760 & 0.774 \\
\hline
\end{tabular}

Table 1lc. SENT Specimen $\mathrm{H}-6, \mathrm{a} / \mathrm{W}=0.222$

\begin{tabular}{lll}
\hline Replica No. & Measured & $\begin{array}{c}\text { CTOD (mm) } \\
\text { Computed from Fig. } 19\end{array}$ \\
\hline 7 & 0.290 & 0.264 \\
9 & 0.495 & 0.563 \\
9 & 0.850 & 1.085 \\
\hline
\end{tabular}


4. TITLE AND SUBTITLE

Effect of Crack-Tip Region Constraint on Fracture in the Ductile-to-

Brittle Transition

5. AUTHOR(S)

T. L. Anderson

6. PERFORMING ORGANIZATION (If joint or other than NBS, see instructions)

MATIONAL BUREAU OF STANDARDS

DEPARTMENT OF COMMERCE

WASHINGTON, D.C. 20234

9. SFONSORHAG ORGANIZATIOH IIAGE AND COHIFLETE ADORESS (Street, C(ty, Stute, ZIF)

7. Contract Grant No.

U.S. Department of Interior, Minerals Management Service

12203 Sunrise Valley Dr.

Reston, VA 22091

10. SUPPLEMENTARY NOTES

[D Document describes a computer program; SF-185, FIPS Software Summary, is attached.

11. ABSTRACT (A 200-word or less factual summary of most significant information. If document includes a significant bibliography or literature survey, mention it here)

The effect of geometry on fracture toughness of steel in the ductile-to-brittle transition region has been studied. The critical crack-tip opening displacement (CTOD) and the critical J-integral have been measured as a function of temperature for ten fracture specimen configurations of ABS grade EH36 steel. In addition, two specimen geometries of commercially pure iron were tested. Thicker specimens tend to promote a higher degree of triaxiality near the crack-tip, thereby elevating the flow stress and making it easier to reach the cleavage stress. This causes a shift in the ductile-to-brittle transition to higher temperatures. Decreasing the uncracked ligament length of bend specimens also tends to shift the transition to higher temperatures. Changing the loading conditions from bending to tension tends to shift the transition to lower temperatures when the ligament is relatively short. When the ligament length is large the transition-region fracture behavior of a bend specimen approaches that of a tension specimen with a similar geometry. A technique was developed to experimentally measure crack-tip constraint. Constraint decreased with crack blunting. This relaxation in constraint was modeled by a simple spring analog. The model was used to predict ductile-to-brittle transition curves for hypothetical structures. Some of the applications and limitations of the model are discussed.

12. KEY WORDS (Six to twelve entries; alphabetical order; capitalize only proper names; and separate key words by semicolons) crack-tip opening displacement; crack-tip constraint; ductile-to-brittle; transition; elastic-plastic fracture; J-integral; mechanical properties; structural steels; toughness.

13. AVAILABILITY

$[X]$ Unlimited

For Official Distribution. Do Not Release to NTIS

Order From Superintendent of Documents, U.S. Government Printing Office, Washington, D.C. 20402.

14. NO. OF PRINTED PAGES

\section{2}

15. Price

$\$ 19.00$ 

